\title{
Cognitive Skill Training for Nuclear Power Plant Operational Decision Making
}

Manuscript Completed: May 1994

Date Published: June 1994

Prepared by

R. J. Mumaw, D. Swatzler, E. M. Roth, W. A. Thomas*

I. Schoenfeld, NRC Project Manager

Westinghouse Electric Corporation

1310 Beulah Road

Pittsburgh, PA 15235

\section{Prepared for}

Division of Systems Research

Office of Nuclear Regulatory Research

U.S. Nuclear Regulatory Commission

Washington, DC 20555-0001

NRC FIN L1653

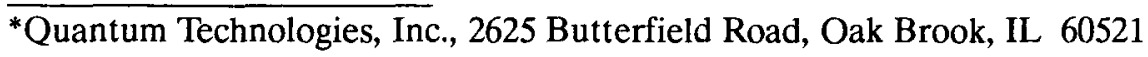




\section{DISCLAIMER}

This report was prepared as an account of work sponsored by an agency of the United States Government. Neither the United States Government nor any agency Thereof, nor any of their employees, makes any warranty, express or implied, or assumes any legal liability or responsibility for the accuracy, completeness, or usefulness of any information, apparatus, product, or process disclosed, or represents that its use would not infringe privately owned rights. Reference herein to any specific commercial product, process, or service by trade name, trademark, manufacturer, or otherwise does not necessarily constitute or imply its endorsement, recommendation, or favoring by the United States Government or any agency thereof. The views and opinions of authors expressed herein do not necessarily state or reflect those of the United States Government or any agency thereof. 


\section{DISCLAIMER}

Portions of this document may be illegible in electronic image products. Images are produced from the best available original document. 


\section{THIS PAGE WAS INTENTIONALLY LEFT BLANK}




\begin{abstract}
Training for operator and other technical positions in the commercial nuclear power industry traditionally has focused on mastery of the formal procedures used to control plant systems and processes. However, decisionmaking tasks required of nuclear power plant operators involve cognitive skills (e.g., situation assessment, planning). Cognitive skills are needed in situations where formal procedures may not exist or may not be as prescriptive, as is the case in severe accident management (SAM).

The Westinghouse research team investigated the potential cognitive demands of SAM on the control room operators and Technical Support Center staff who would be most involved in the selection and execution of severe accident control actions. A model of decision making, organized around six general cognitive processes, was developed to identify the types of cognitive skills that may be needed for effective performance. Also, twelve SAM scenarios were developed to reveal specific decision-making difficulties. Following the identification of relevant cognitive skills, 19 approaches for training individual and team cognitive skills were identified. A review of these approaches resulted in the identification of general characteristics that are important in effective training of cognitive skills.
\end{abstract}




\section{THIS PAGE WAS INTENTIONALLY LEFT BLANK}


Table of Contents

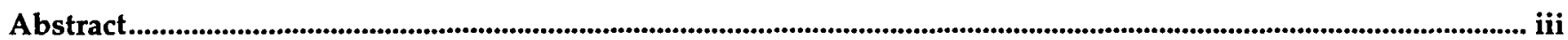

Executive Summary

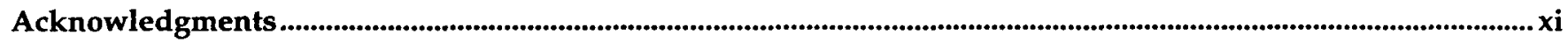

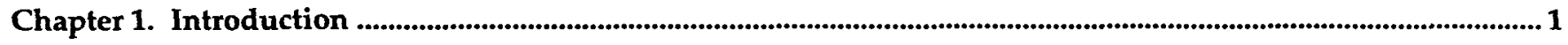

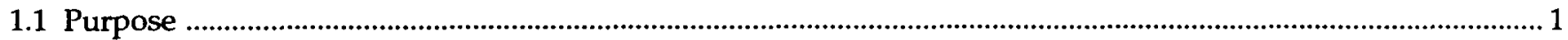

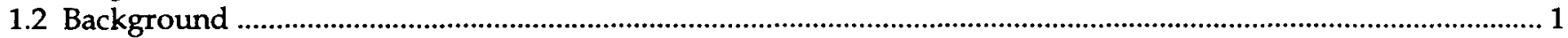

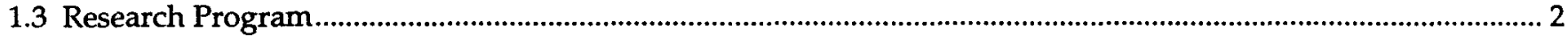

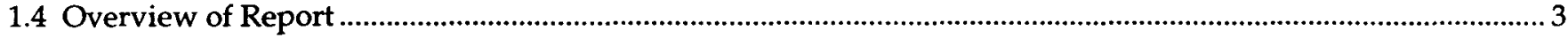

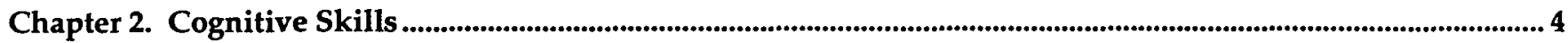

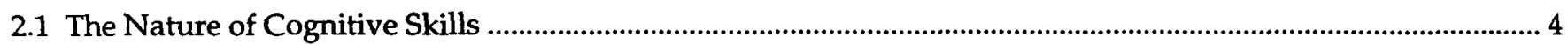

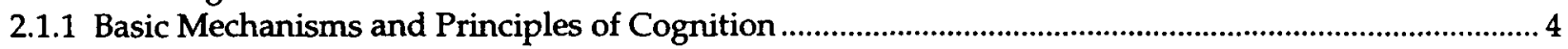

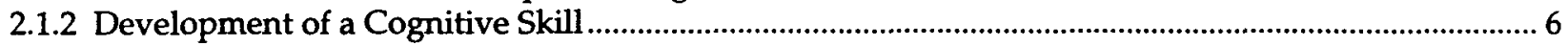

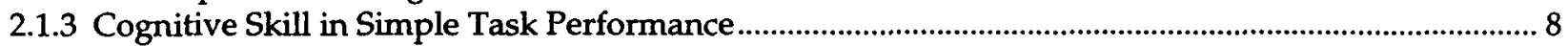

2.1.4 Cognitive Skill in Complex Task Performance ......................................................................................... 10

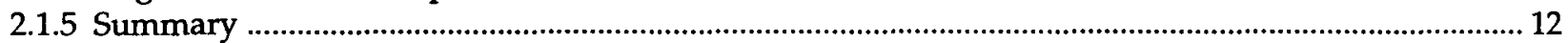

2.2 Related Concepts

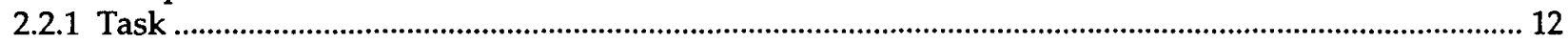

2.2.2 Knowledge-Based, Rule-Based, and Skill-Based ..................................................................................... 13

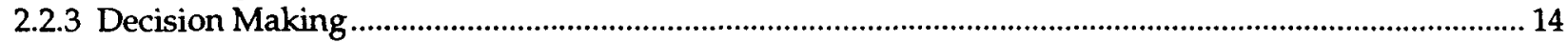

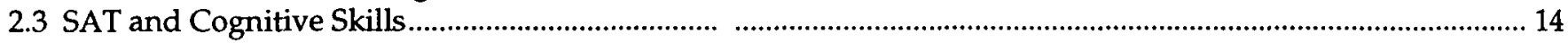

Chapter 3. Complex Decision-Making Tasks in Nuclear Power Plants ................................................................ 15

3.1 Cognitive Skills in Decision Making During Routine and Emergency Operations .......................................... 15

3.2 The Special Case of Severe Accident Management ..................................................................................................... 18

Chapter 4. A Model and Analysis of NPP Decision Making ...................................................................................... 21

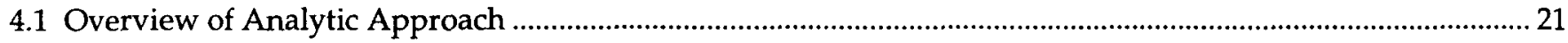

4.2 Model of Operator and Technical Staff Decision Making …............................................................................... 24

4.2.1 Performance as Decision Making ........................................................................................................... 24

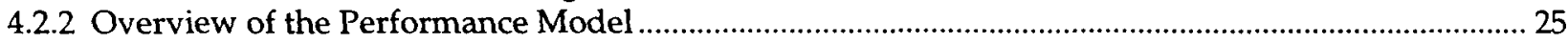


Table of Contents (continued)

Chapter 5. Cognitive Skills Required for Decision Making …................................................................................39

5.1 Identification of Cognitive Skills from Analysis of the Performance Model ..................................................... 39

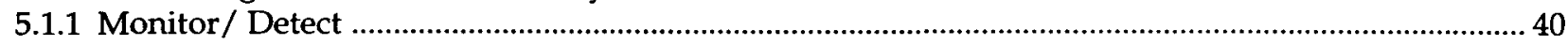

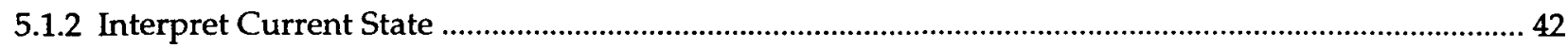

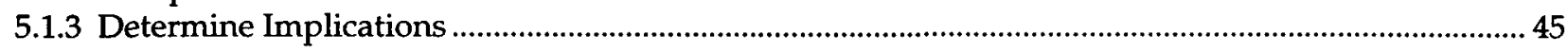

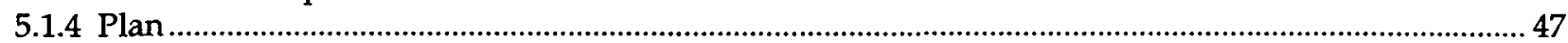

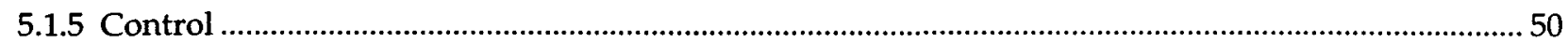

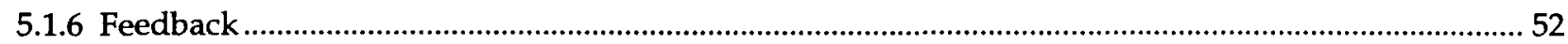

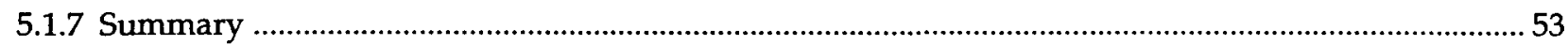

5.2 Identification of Cognitive Skills from Analysis of SAM Scenarios ..............................................................5

Chapter 6. Cognitive Skill Training: Principles and Techniques ............................................................................... 57

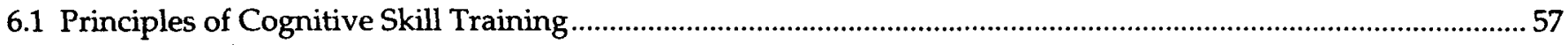

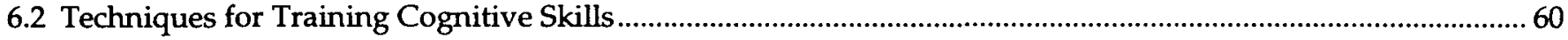

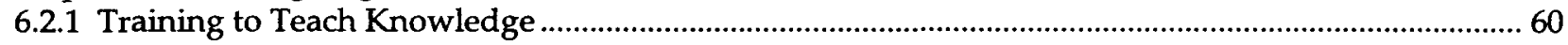

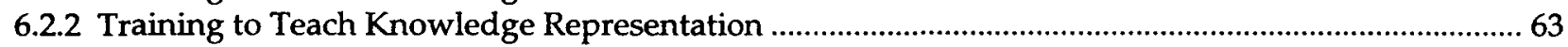

6.2.3 Training to Teach Rules Applied to Decision Making ..............................................................................64

6.2.4 Training to Teach Strategies, Goals, and Subgoals .............................................................................64

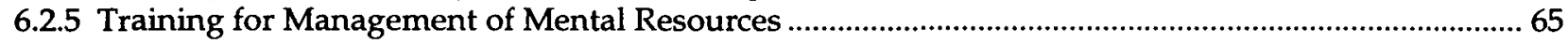

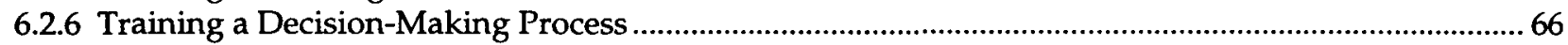

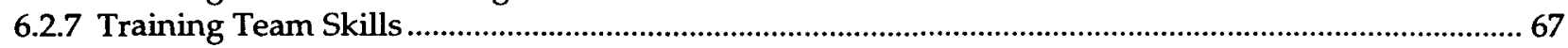

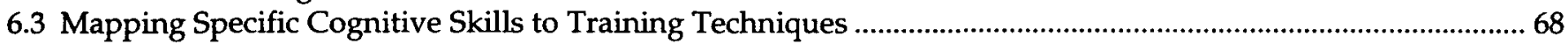

6.4 Characteristics of Effective Training of Cognitive Skills ............................................................................... 70

6.5 Cognitive Skills and Alternatives to Cognitive Skill Training ............................................................................. 71

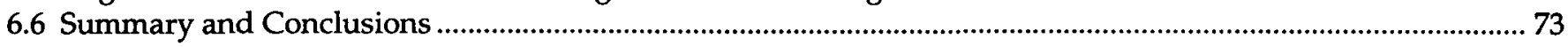

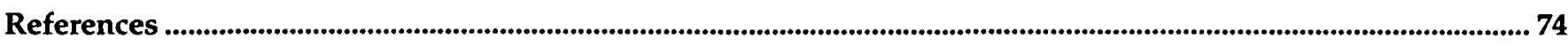

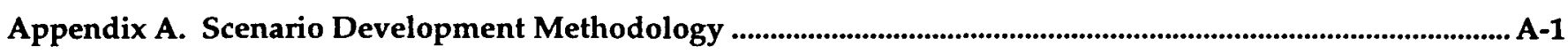

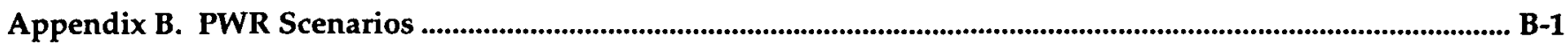

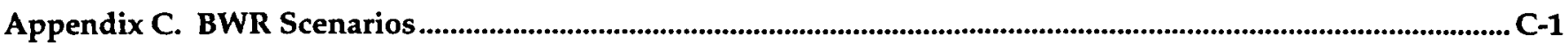

Appendix D. Training Techniques - Research and Discussion ............................................................................... 


\section{List of Figures}

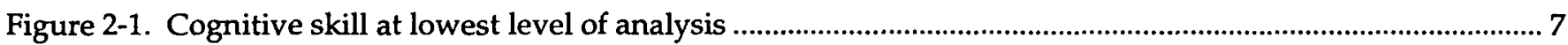

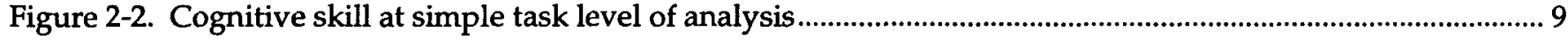

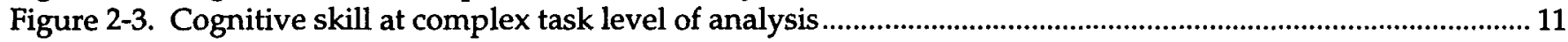

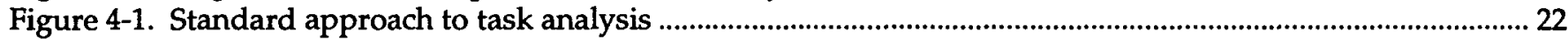

Figure 4-2. Schematic of the analytic approach to the identification of cognitive skills ..........................................2 23

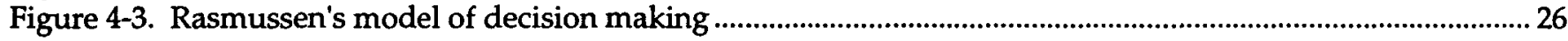

Figure 4-4. The six general components of the performance model ...................................................................... 27

Figure 4-5a-b. Expanded versions of the six general performance model components .............................................2.28

Figure 4-5c-d. Expanded versions of the six general performance model components ..............................................30

Figure 4-5e-f. Expanded versions of the six general performance model components ............................................. 32

Figure 4-6. Integration of expanded performance model................................................................................................... 34

Figure 4-7. Important transition points between processes ................................................................................... 35

Figure 4-8. Performance model components used for simplest rule-based decision

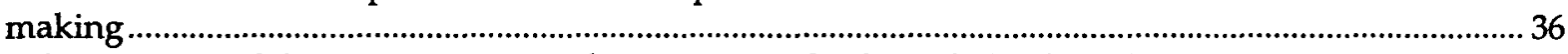

Figure 4-9. Performance model components used for most complex knowledge-based

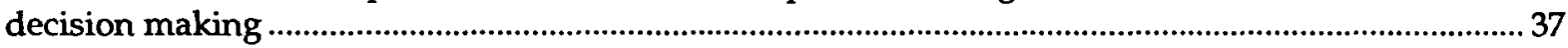

\section{List of Tables}

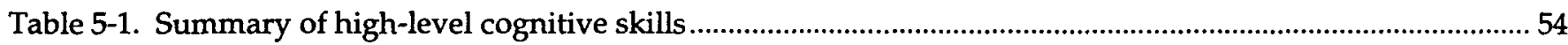

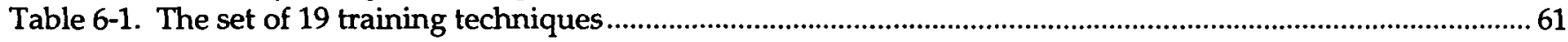

Table 6-2. Assignment of cognitive skills to training technique classes....................................................................69

Table 6-3. Important training characteristics for each training technique ……........................................................... 72 


\section{THIS PAGE WAS INTENTIONALLY LEFT BLANK}




\section{Cognitive Skill Training for Nuclear Power Plant Operational Decision Making}

\section{Executive Summary}

Training for operator and other technical positions in the commercial nuclear power industry traditionally has focused on mastery of the formal procedures used to control plant systems and processes. However, there is a growing awareness that the decisionmaking tasks required for selecting appropriate control actions, in addition to guidance from formal procedures, also involve cognitive activities commonly referred to as judgment or reasoning. The need for cognitive skills is especially clear in situations where formal procedures may not exist or may not be as detailed. One example of this situation is severe accident management (SAM), which addresses accidents that involve overheating of the core beyond the design basis. Thus, for these cases training for decision-making tasks should consider the development of appropriate cognitive skills.

This report has the following objectives:

- Define cognitive skills and describe why cognitive skills are often not addressed well by current training programs.

- Provide an analysis and model of decision making in the nuclear power plant (NPP) setting, addressing both rule-based and knowledge-based performance.

- Determine the cognitive skills that are critical to NPP decision making, especially in the case of SAM.

- Identify important characteristics of cognitive skill training.

- Describe specific training techniques that could be applied effectively to the cognitive skills required for decision making.

Chapter 1 provides an introduction that includes this project's purpose and an overview of the complete report. Chapter 2 presents the fundamental mechanisms of cognition and describes cognitive skills at three levels of analysis. The critical elements of cognitive skill identified are:

- Task-relevant knowledge.
- Meaningful organizations of knowledge (mental representations).

- Task-relevant cognitive processes (rules) that can be used to modify the representation.

- The set of task subgoals that provide efficient paths to the goal.

- The strategies (strings of rules) that can be used to achieve subgoals.

- Metacognitive skills that guide the selection and execution of individual skills.

Chapter 3 reviews the results of studies of NPP operator performance during emergency conditions to identify cognitive skills that are relevant and important to performance. This review suggests that crews use cognitive skills even when procedures are available (rule-based performance). In particular, crews engage in situation assessment, which allows them to identify relevant parameters and indications and allows them to anticipate and monitor related phenomena. Also derived from the situation assessment, when linked to knowledge of the procedures, is a clear understanding of the goals and subgoals that need to be achieved. More peripheral are cognitive aspects of selecting and executing control actions. For example, potential undesirable side effects of control actions can be assessed within the mental representation before an action is actually taken.

The following are potential issues for SAM that are related to an increased need for cognitive skills:

- Decision-making authority may be transferred from the control room and Technical Support Center, which leads to an increased reliance on communication.

- Reliable and accurate plant state data will be more difficult to obtain.

- SAM guidance documents may not be prescriptive to the same degree as current emergency procedures.

- Uncertainty in decision making will be increased.

- Stress levels on decision makers will be increased. 
Chapter 4 presents a model of decision making that is used to identify the specific cognitive skills that may be required for SAM. The model uses six general processes:

- Monitor / Detect - active (monitor) and passive (detect) means for acquiring data about plant state.

- Interpret Current State - the development of a mental representation of plant status.

- Determine Implications - the determination of how the current plant state will progress (e.g., potential consequences, side effects). Also, a set of goals is defined in which more important goals are given higher priority and complex goals may be broken down into subgoals.

- Plan - the selection of a response plan, which could be a high-level action or formal procedure, that addresses the goal(s) with the highest priority.

- Control - the coordination and execution of a specific sequence of control actions.

- Feedback - the information gained from control actions is used to update understanding.

Chapter 5 presents each of these processes and their associated cognitive skills in the context of SAM. Also, 12 SAM scenarios are described that reveal potential decision-making difficulties and the necessary cognitive skills. These 12 scenarios, six PWR and six BWR, are presented in Appendices B and $C$.

Chapter 6 addresses training techniques that are relevant to the types of cognitive skills identified in our analysis of SAM decision making. Chapter 6 first establishes a broad context, not specific to NPP decision making, for the effective training of cognitive skills. The intent is to describe an effective approach to training complex, cognitive skills--an approach that connects the mechanisms underlying cognitive skills and the techniques for training them. A set of principles to structure a training program is presented.

Chapter 6 then presents 19 techniques for training cognitive skills or facilitating the training of cognitive skills for individuals and teams of individuals. Different classes of training techniques to address the different cognitive skills identified in Chapter 2 are described. These types are:
- Training to teach knowledge

- Training to teach knowledge representation

- Training to teach rules applied to decision making

- Training to teach strategies, goals, and subgoals

- Training for management of mental resources

- Training a decision-making process

- Training team skills

A mapping between the cognitive skills identified in Chapter 5 and the classes of cognitive skill training is then presented. It is not within the scope of this report to design an ideal approach for training the cognitive skills identified in the analysis of SAM. Instead, general characteristics that are critical to effective training of cognitive skills are identified. At least five important characteristics emerge throughout the approaches described, and they appear in the discussions of learning principles. These characteristics are:

1. A model of skilled or expert performance to be used to establish concrete training objectives and as a diagnostic aid in training.

2. Involvement of trainees in evaluating their performance (or the performance of others) using as a standard the model of skilled performance.

3. Active engagement of trainees in the task as a setting for instruction.

4. A method to support trainee performance to allow them to be involved (eventually) in performance of the complete task.

5. A method to aid trainees in managing mental workload throughout training.

Each of these characteristics is described in more detail and illustrated through the specific techniques reviewed in Chapter 6. 


\section{Acknowledgments}

The authors wish to take this opportunity to thank Isabelle Schoenfeld and J.J. Persensky of the Human Factors Branch, Office of Nuclear Regulatory Research, for defining and maintaining the overall program objectives and ensuring that our project deliverables remained responsive to NRC's needs. Isabelle strongly influenced the organization and direction of the project reports. Further, Isabelle ensured that we coordinated our activities appropriately with relevant efforts in the Office of Nuclear Reactor Regulation (NRR) at the NRC.

We also wish to acknowledge the efforts of David DeSaulniers of the Human Factors Assessment Branch, NRR, and Robert Palla of the Probabilistic Safety Assessment Branch, NRR. David represented NRR Human Factors considerations for the program in its initial definition and as a reviewer. Robert Palla provided the context of NRC's Severe Accident Management program and aided us in verifying technical details.

Finally, we thank Alan Lesgold, Professor of Psychology and Associate Director of the Learning Research and Development Center at the University of Pittsburgh, for reviewing our discussions of cognitive skill acquisition and training. Professor Lesgold has helped to shape this field over the last 15 years, and his work has strongly influenced our approach to the subject. 


\section{Chapter 1 \\ Introduction}

\subsection{Purpose}

Training for operator and other technical positions in the commercial nuclear power industry traditionally has focused on mastery of the formal procedures used to control plant systems and processes. However, there is a growing awareness-e.g., a report from the NRC's Office for Analysis and Evaluation of Operational Data (Kauffman et al., 1992)--that the decision-making tasks required for selecting appropriate control actions also involve cognitive skills, commonly referred to as judgment or reasoning. The need for cognitive skills is especially clear in situations where formal procedures may not exist or may not be as detailed, as is the case in severe accident management (SAM). Thus, training for decision-making tasks should consider the development of appropriate cognitive skills. This report describes work in the area of cognitive psychology that relates to nuclear power plant decision-making tasks, the types of cognitive skills required for these tasks, and the types of training techniques that are most appropriate for these skills.

\subsection{Background}

The commercial nuclear power industry and the Nuclear Regulatory Commission (NRC) have tried to anticipate potential nuclear power plant (NPP) failures that could pose a threat to the environment and the general public. Indeed, safety concerns have been a significant component of plant licensing, plant operation, and personnel training. At one time, single-failure events, such as a steam generator tube rupture, defined the utilities' safety and emergency planning and training. The 1979 incident at Three Mile Island's unit 2 (TMI-2) brought about an evolution in thinking about NPP failures; TMI-2 was a watershed event that made the industry re-think the way it planned for and handled accidents. In the TMI-2 event, a sequence of failures and operator errors contributed to the delayed recovery and eventual severity. In the ensuing years, Significant Event Reports (SERs) and Licensee Event Reports (LERs)-which are utility-generated reports on abnormal events in plant operation (e.g., a violation of the limiting conditions of operation)-- indicated that multiple operational errors and system malfunctions are less rare than the industry had at one time believed. Further, multiple-failure accidents in other industries led several authors (e.g., Perrow, 1984; Reason, 1990) to comment on the significance and potential risk associated with multiple-failure events.

It also became understood that, in many of the NPP multiple-failure cases, the occurrence of an additional fault or operator failure could have pushed the affected plant beyond its ability to maintain public safeguards. Thus, over the last ten years, the NRC, the commercial nuclear power industry, and other concerned organizations (e.g., EPRI, DOE) have invested significant resources, both human and monetary, to improve the reliability and performance of nuclear power plants during normal, off-normal, and emergency conditions. Upgrades and backfits have included hardware and software systems, maintenance programs, alarm systems, emergency operating procedures, human factors engineering, personnel training, staffing, and personnel qualifications. These improvements have significantly enhanced plant safety and have effectively reduced potential health risks to workers and the public. While these changes provide a stronger defense against severe safety challenges, a potential still exists for multiple failures of equipment or personnel that can result in a severe accident and substantial health risks (cf. NUREG 1150). Although these risks are extremely low, the NRC initiated several joint NRC-industry programs to ensure the industry's ability to manage severe accidents effectively. These programs have focused on the development of mitigation strategies and guidance for operational decision-making .

Generally, a severe accident is defined as one that involves overheating of the core beyond the design basis. The NRC's primary action to ensure that severe accident management (SAM) is addressed by each utility is described in Generic Letter 88-20 and NUREG 1335, which address Individual Plant Examinations (IPEs), and the Accident Management 
Program, as defined by SECY-89-012. Under the Accident Management Program, each utility was asked to submit an Accident Management Plan that addresses the following elements:

- Accident management strategies.

- Instrumentation.

- Guidance (procedures) and analysis aids.

- Decision-making process.

- Training.

These five elements provide a framework for analyzing and improving plant safety and personnel performance during a severe accident. The IPEs, which are currently being completed by utilities, are the primary vehicle for determining what event sequences can lead to core melting, reactor vessel failure, and eventually containment breach. Thus, the IPEs will become the basis for identifying the plant-specific details for each utility's Accident Management Plan.

The inclusion of the decision-making process and training as elements of the Accident Management Plan reveals the contributions the "human system" can provide. The operating crew, technical staff, utility management, and other personnel associated with nuclear power plants can take actions to prevent accidents from progressing to core damage or to mitigate the consequences of a severe accident after it has occurred. However, human behavior and response during severe accident conditions may differ from that which is expected during anticipated abnormal operations. Therefore, training that focuses on decision making under severe accident situations may be a viable means of improving the effectiveness and reliability of human performance under such conditions. There are two critical elements to this type of decision making that set it apart from decision making during normal and abnormal situations: the strong requirement for cognitive skills and the high likelihood of excessive stress.

\subsection{Research Program}

The NRC initiated a program to identify viable training techniques for the special skills required for decision making during severe accident management-in particular, cognitive skills and skills for coping with high levels of stress. This program accomplished the following:

1. Reviewed the current state of severe accident management programs. A review of documents and technical reports and interviews with experts were conducted to determine the current state of U.S. (and some foreign) severe accident programs. Information was collected on how the industry is planning to support SAM, what difficulties they think will need to be addressed, and what training they believe will be required. Also, on-going efforts to develop severe accident management guidance (SAMG) documents that will support SAM decision making were reviewed.

\section{Reviewed relevant task analyses and training} programs in the nuclear and related industries. Task analyses within the nuclear power industry that address decision making and accident management were identified.

3. Developed severe accident scenarios to identify potential decision-making difficulties. To better understand the decision-making task required for SAM, experts developed a set of realistic severe accident scenarios. Six scenarios were developed for PWR plants and six for BWR plants. These scenarios revealed the types of difficulties that may arise during SAM.

4. Developed an analytic model of decision making under severe accident conditions to identify cognitive skills. A general model of human decision making that is widely used for describing NPP operator performance was selected. This model was modified to address the special case of SAM. From this model, the cognitive skills required to a) perform decisionmaking tasks at a high level of skill, and b) reduce errors and inefficient performance were identified. These skills were defined for individual and crew performance.

5. Reviewed the scientific and technical literature on decision making performance. A literature review was conducted of a) models and theories of decision making, b) empirical evidence of errors and biases in decision making, and c) NPP operator performance in difficult decision-making tasks.

6. Reviewed the scientific and technical literature on training cognitive skills. A review was conducted 
of the types of training techniques that have been applied to cognitive skills of the type required for decision making. Also, existing training programs within the nuclear industry, within other safetyrelated industries, within the Department of Defense, and from academic settings that may be relevant to training decision making skills in similar contexts were reviewed.

7. Reviewed the scientific and technical literature on the effects of stress on performance and decision making. A review was conducted of a) theories of stress and its general effects on human performance, and b) empirical investigations of the effects of stress on performance of cognitive tasks.

\section{Reviewed the scientific and technical literature} on training stress-coping skills. Literature on the types of training approaches that have been used to enhance stress-coping skills was reviewed.

Thus, although the program's initial focus was on SAM and analyses of severe accident decision making were conducted, this report and its companion report address more broadly the decision making tasks and cognitive skills required for NPP operations and accident management. The title of the companion report (NUREG/CR-6127) is "The Effects of Stress on Nuclear Power Plant Operational Decision Making and Training Approaches to Reduce Stress Effects." Severe accident management serves as an illustration of decision making under stress in the NPP setting.

\subsection{Overview of Report}

This report has the following objectives:

- Define cognitive skills and describe why cognitive skills are often not addressed adequately in training programs.

- Provide an analysis and model of decision making in the NPP setting, addressing both rule-based and knowledge-based performance.

- Determine the cognitive skills that are critical to NPP decision making, especially in the case of severe accident management.

- Identify important characteristics of cognitive skill training.
- Describe specific training techniques that could be applied effectively to the cognitive skills required for decision making.

Chapter 2 defines cognitive skills and other concepts relevant to this report, such as knowledge-based performance, rule-based performance, and decision making. This section also discusses the reasons that traditional training analysis and development have not adequately addressed cognitive skills.

Chapter 3 discusses decision making in NPP operations. First, decision making under normal and abnormal conditions is described to identify the role of cognitive skills in rule-based performance. Then, the special case of severe accident management (SAM) and the additional difficulties that may be introduced to NPP decision-making tasks are described.

Chapter 4 describes an approach for analyzing decision making to identify the cognitive skills. This section includes a detailed model of human performance for NPP decision-making tasks. This model is adapted from other work in the analysis of operator performance to address both rule-based and knowledge-based forms of decision making, especially as in the case of SAM.

In Chapter 5, the performance model is used to identify critical cognitive skills required for decision making. In this chapter, a set of 12 severe accident scenarios is introduced that was created to aid our analysis of decision making.

Chapter 6 describes general principles of training that support the learning of cognitive skills. That is, there are principles that guide the structuring of the entire training program in addition to specific training techniques directed at individual skills. These principles, largely taken from research in cognitive psychology, can help shape a training program that makes learning more efficient and increases skill levels. This chapter also reviews specific training techniques that can be applied to the types of cognitive skills identified in Chapter 5. Each training technique is described along with examples of its application, theoretical underpinnings, data on effectiveness, etc. This chapter concludes by presenting a list of qualities that best describe effective techniques for training cognitive skills. 


\section{Chapter 2 Cognitive Skills}

A number of terms that are central to this report may be unfamiliar to some audiences. Hence, the following sections provide a brief discussion of cognitive skills, decision making, and related concepts.

\subsection{The Nature of Cognitive Skills}

Over the last 15 years, the importance of cognitive skills for certain types of tasks has become more apparent. Research in the fields of cognitive psychology and instructional design and technology have provided training methods and theories that are specifically tailored to cognitive skills. This section is an overview of some of the essential concepts used for describing cognitive skills.

Cognitive skills are concerned with how individuals acquire, store, and use knowledge. These skills are used to solve problems, make decisions, make inferences, reason about the effects of inputs to a system or process, etc. This characterization (store and use knowledge) draws a primary distinction in discussing cognitive skills: the distinction between representation of knowledge and processing of knowledge. Representation refers to how knowledge is stored in memory. Processing refers to how knowledge is retrieved from memory and applied to a task or used to create new knowledge (e.g., inferences). Both elements of cognitive skill are important to the development of a high skill level.

\subsection{Basic Mechanisms and Principles of Cognition}

Cognitive psychology has established the primary importance of mental structures. Humans break up the world into common objects (e.g., a person, a face, a piece of furniture) and events, where an event in this context refers to a meaningful series of actions over time (e.g., a meal, a trip to work). When novel objects or events occur, humans attempt to interpret them in terms of known entities or known scenarios. Because of this, cognition is described as a combination of data-driven and knowledge-driven processes. Data-driven processes refer to taking in and interpreting information from the world. Knowledge-driven processes refer to attempts to fit existing notions of the world onto the incoming information. The knowledge-driven processes create expectations about what will be present since each object or event is defined by a set of typical characteristics. Hence, humans may miss information that is unexpected (information that does not fit the typical case), and they may add information that was not present. The data-driven processes provide the clues about which event is actually occurring and how well it matches the generic version stored in memory. However, the amount of information in the world can be overwhelming, and knowledge-driven processes, by directing attention, aid in selecting information that may be relevant.

A common example is the series of actions associated with going to a restaurant for dinner. This event is familiar to most people in our culture, and we have a set of expectations about what will occur: we will be seated, we will be given menus, we will give an order to a waiter or waitress, etc. Our understanding of this event is captured in a mental structure called a schema (sometimes called a script). Our restaurant schema, like all schemas, has a set of "defaults" that represent our expectations. These expectations can be so strong that they can distort our memory of what actually took place in the restaurant (Loftus, 1982). For example, we may eventually forget that individual menus were not provided because a large menu hung on the wall.

In a nuclear power plant setting, the implication is that experienced operators have schemas for common events or well-rehearsed accident scenarios. When initial symptoms are recognized as indicative of a familiar event, knowledge-driven processing takes a significant role in performance. Operators have expectations about what will occur next, which can determine which indications they seek and affect their interpretation of ambiguous information (e.g. is parameter $\mathrm{P}$ stable?). Or, the schema may influence an operator to ignore or discount information that does not fit expectations, or 
perhaps "fill in" details that were not actually present. The use of schemas is pervasive and guides virtually all behavior. Even when events occur that are relatively novel, humans use some general set of expectations to guide performance.

Knowledge is stored in what is called long-term memory (LTM), which is believed to be virtually unlimited. Knowledge is organized in LTM in several ways. First, as just described, knowledge is organized as schemas that help one interpret the world. A second form of memory organization are "mental models," which are incomplete and often flawed models of the world (Gentner \& Stevens, 1983). These complex representations allow one to simulate a system or process mentally in order to reason about cause and effect, consequences of actions, feasibility of control actions, effects of malfunctions or failed components, etc. These models typically capture both physical and functional characteristics of a system or process. However, mental models often contain gaps or misconceptions. In some cases, multiple models may exist with each representing partially overlapping, or sometimes nonoverlapping, components of a system. Also, a mental model may facilitate reasoning about some phenomena but provide no assistance in reasoning about other phenomena (Gentner \& Gentner, 1983).

Factual knowledge may also be stored in less complex organizations, such as simple relationships (e.g., $x$ is an instance of $y, a$ is a special case of $b, q$ has characteristic p, etc.). Another important distinction exists between the type of knowledge described so far, which is referred to as declarative knowledge, and procedural knowledge (Anderson, 1983). Procedural knowledge is thought to be stored as sets of conditional statements in the form of "if $x$ occurs, then do $y . "$ These if-then pairs are called productions.

Information is brought (either from the world or from LTM) into a temporary workspace, referred to generally as working memory (WM), or active memory. One critical aspect of WM is that, unlike LTM, it has limitations on the amount of information it can hold. The number of items that can reside in WM at one time is limited to about seven (Miller, 1956). When humans attempt to keep active many more than seven separate items (or when there are a number of manipulations of a single item), information is displaced or lost. One can increase the amount of information available from WM, though, by increasing the size of an item. Thus, even though there seems to be a limit on the number of items, the size of an item can vary. One can increase the size of an item by constructing meaningful "chunks" of information associated with a single label. For example, if asked to recall a random string of numbers that are read aloud, most individuals will have trouble recalling more than about seven numbers. However, if those seven numbers represent one's telephone number, which is a meaningful "chunk," the numbers can be assigned that label and stored in WM as a single chunk. The seven individual digits do not need to occupy seven separate slots in WM since one's telephone number is easily retrieved from LTM-that is, the label stored in WM is used to retrieve the specific information from LTM. Taking this to the extreme, working memory, though limited, can store a large amount of information by storing the labels of large, meaningful chunks of information.

A second limitation of cognitive processing is in the gathering of information from the world. Attention is the mechanism through which information in the world is selected and brought into WM. Obviously, there are limits on how much information can be attended to at one time. In knowledge-driven processing, one directs attention consciously to certain areas of the world that are likely to provide relevant information. In other cases, attention is directed by stimuli in the world. Primarily, the stimuli that capture one's attention are those that have meaning at that particular moment (e.g., one's name, information related to the issue one is thinking about) and those that are particularly salient (e.g., loud stimuli, bright stimuli, flashing stimuli).

Thus, although LTM is considered to be virtually unlimited, the ability to access information from the world and the ability to hold and manipulate information in WM are strongly limited. As just described, the use of chunking is a method that can reduce those limitations. Another means for reducing the effects of cognitive limitations is to develop cognitive processes to the point where the requirements for mental resources are greatly reduced. Schneider \& Shiffrin (1977) determined that certain types of cognitive skills, through extensive practice, can become "automated." That is, they can be performed with almost no commitment of 
mental resources. Other processes cannot achieve this status; they are referred to as "controlled" processes since they rely on conscious control of mental resources. A familiar example of automated processes are typing skills. A skilled typist can type accurately with little reliance on mental resources, which may be reserved instead for deciphering and comprehending a complex, hand-written manuscript. Other skills that illustrate the development of automated skills in adults are reading (decoding words) and driving a car (steering, braking, shifting). There are strong differences between the types of processes that can be automated and the types of processes that must remain controlled. A careful task analysis can isolate the processes that can become automated, and training can be used to reduce their requirement for mental resources.

Also related to the use of limited resources is the notion of metacognitive processes, which are higherlevel processes that initiate and guide cognitive activities. These metacognitive processes--such as monitoring the progress of cognitive activities, directing attention, selecting methods for memorizing or learning or problem solving-control the commitment and use of limited cognitive resources for attention and WM. One familiar element of this aspect of cognition is metamemory. Adults, unlike young children, are typically skilled at assessing their ability to commit information to memory. Thus, when an adult is required to recall at a later time a great deal of information (e.g., shopping list), he is likely to write it down or take a lot of effort to memorize it. There is an understanding of capability limitations and of effective means to overcome the limitation (i.e., mnemonics, writing on a piece of paper, string around the finger). This "management" function, which develops in late childhood, is part of metacognition.

The preceding discussion characterizes the basic principles and mechanisms of cognition: the distinction between representation and processing, the importance of knowledge organization, knowledge-driven vs data-driven, limitations on working memory and attention and means for reducing their effects, and the role of metacognitive processes. From this foundation, one can now develop a description of the development of cognitive skills. The basic conception of cognitive skills is that information is taken from LTM or from the world (or both) and manipulated in some way in WM to produce new knowledge, which is then used to act on the world.

\subsubsection{Development of a Cognitive Skill}

Several researchers (e.g., Carroll, 1976, Fleishman, 1982) have attempted to classify the set of manipulations (or cognitive processes) that are applied to knowledge. Sticha, Schlager, Buede, Epstein, and Blacksten (1990) reviewed classification schemes from a number of theorists and developed a set of 16 basic cognitive processes; two examples are deductive reasoning and analogical reasoning. Although a classification scheme can be useful for systematic analyses of cognitive tasks, there is currently no universally agreed-upon scheme. However, there is a strong consensus on how cognitive processes are incorporated as a cognitive skill is developed.

Current theories use a three-stage model to describe cognitive skill acquisition (Fitts, 1964; Anderson, 1983). As described above, before specific skill development begins, a set of basic cognitive processes are available that can be applied to any knowledge. These processes are often stated as production rules of the form "If $x$, then $y$." For example, "If $A$ is equivalent to $B$ and $B$ is equivalent to $C$, then $A$ and $C$ are equivalent." This type of rule can modify the mental representation by making explicit a meaningful relationship between two items. In general, these rules take knowledge that already exists, and manipulate it to produce new knowledge or to update a more complex mental representation (e.g., update a mental model of a system). Thus, the processing enhances (upgrades, improves) the representation (see Figure 2-1).

In the first phase of skill acquisition, the learner acquires job-related knowledge that is used in conjunction with the basic cognitive processes to solve problems or make decisions. Performance in this stage is slow, heavily reliant on consciously controlled processing, and open to introspection (that is, performers can easily explain their performance).

In the second stage of skill acquisition, rules are refined and errors are detected and eliminated. There is a gradual development of task-specific procedures, which are modified versions of the more general cognitive processes. As these processes become refined and more strongly tied to specific task 
PRACTICE

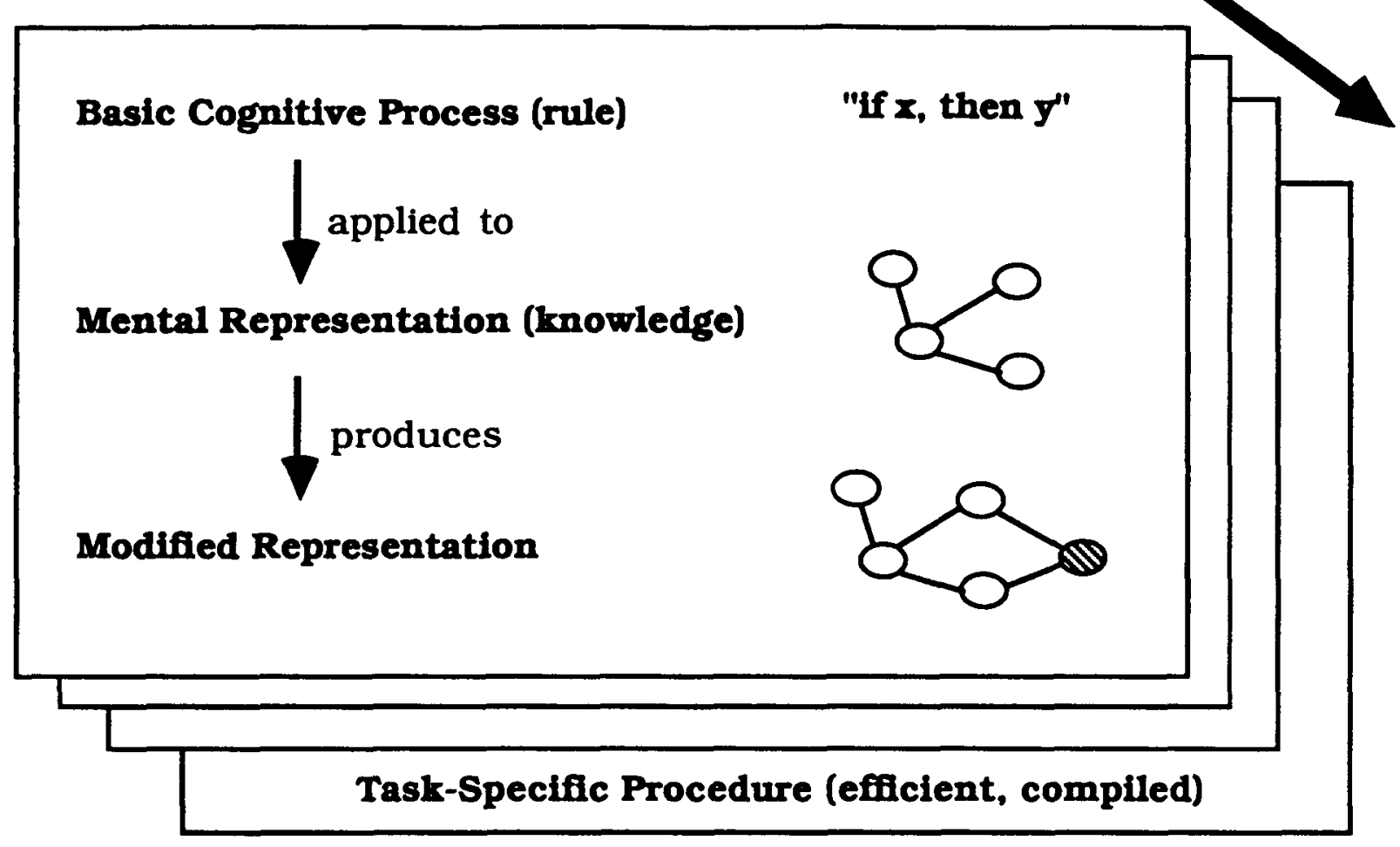

Cognitive Skill is Defined by:

1. Knowledge Organization within Mental Representation

- Mental Models

- Functional Characteristics of Representation

2. Task-Specific Process that Becomes Proceduralized

Figure 2-1. Cognitive skill at lowest level of analysis. 
knowledge, they can become less accessible to introspection. That is, it becomes much more difficult for performers to explain their own performance. In the final stage of acquisition, the cognitive skill becomes even less reliant on conscious processing and can be executed more rapidly. Thus, there is a trade-off between rapid execution and the ability to introspect on performance.

There are numerous examples of this acquisition process in the NPP setting. One example is a PWR operator's ability to quickly determine the magnitude of a leak. A pressurizer level deviation alarm may signal the presence of a leak in the primary system. An operator is trained to assess the magnitude of the leak by gathering and comparing a set of flow rate indications. Specifically, by looking at charging flow rate, letdown flow rate, seal injection flow rate, and seal return flow rate, an operator can compute degree of leakage. Initially, this decision will proceed by gathering and consciously comparing flow rate indications. Processing will be slow and will require most of the trainee's mental resources. Through training, operators become much more efficient by proceduralizing this skill. Eventually, upon hearing the alarm, an operator may quickly scan the set of indicators and judge the leak rate. This process will appear to be almost intuitive, and the operator may have trouble reporting how he arrived at a judgment.

Thus, at the lowest level of analysis (Figure 2-1), a cognitive skill begins with a basic cognitive process and, through the incorporation of task-specific knowledge, adapts that process to perform a task element. There is a gradual change in the process in which a general method for manipulating knowledge becomes more tuned to a specific task and becomes more efficient. Cognitive psychologists who work at this low level of analysis (Anderson, 1983; Kieras and Bovair; 1984) attempt to determine the set of task-specific procedures (rules) required to perform each task element and the characteristics of the mental representation that is used. Because of the fine level of detail required in this level, it is difficult to apply this analysis to operator tasks that are broader in scope (i.e., involve many task elements and cognitive skills). Therefore, the next section places this basic conception of cognitive skills into a broader task framework that introduces the importance of goals.

\subsubsection{Cognitive Skill in Simple Task Performance}

Human cognitive behavior, especially in a task setting, is strongly driven by the identification and achievement of goals. This level of analysis begins to incorporate metacognition-that is, the strategic management of cognitive processes becomes important to performance.

A goal can be defined at many levels; examples are achieving a specific power level, recovering a specific pressure level, recovering a stable plant state. Newell and Simon (1972) developed a framework in which task behavior is directed by successive comparisons between the current state of the world and the desired goal state. For example, an operator might be faced with a parameter value that is different from the desired value, and must determine how to achieve the goal state: How do I achieve this goal using the control actions available to me?

There are a number of key concepts within this framework, which broadens the definition of cognitive skill. As before, the representation of the task and the processes applied to that representation are essential. Thus, the operator must initially develop a mental representation of the current state of the system (called a problem state) and a mental representation of the goal state. By processing a representation (applying a rule), one can modify it to more closely approximate the goal state. Figure 2-2 shows an organization of problem states for a simple problem. Each connection between problem states represents the application of a rule to change the representation. For example (lower left corner of the figure), problem state 1 can be changed into problem state 3 by applying rule $R 1$. If rule $R 2$ is applied to the same problem state, the representation is modified in a different way and problem state 4 is the result. The problem solver's task is to continue to modify the problem state until it is identical to the goal state. As Figure 2-2 shows, there are multiple ways to do that. One method is to apply rules $\mathrm{R} 1, \mathrm{R} 4, \mathrm{R} 7, \mathrm{R} 10, \mathrm{R} 12$, and $\mathrm{R} 14$.

Thus, within this framework, the nuclear power plant operator must have cognitive processes that can be applied to reduce the differences between the representations of the two system states. Basic cognitive processes can be applied, but more valuable 


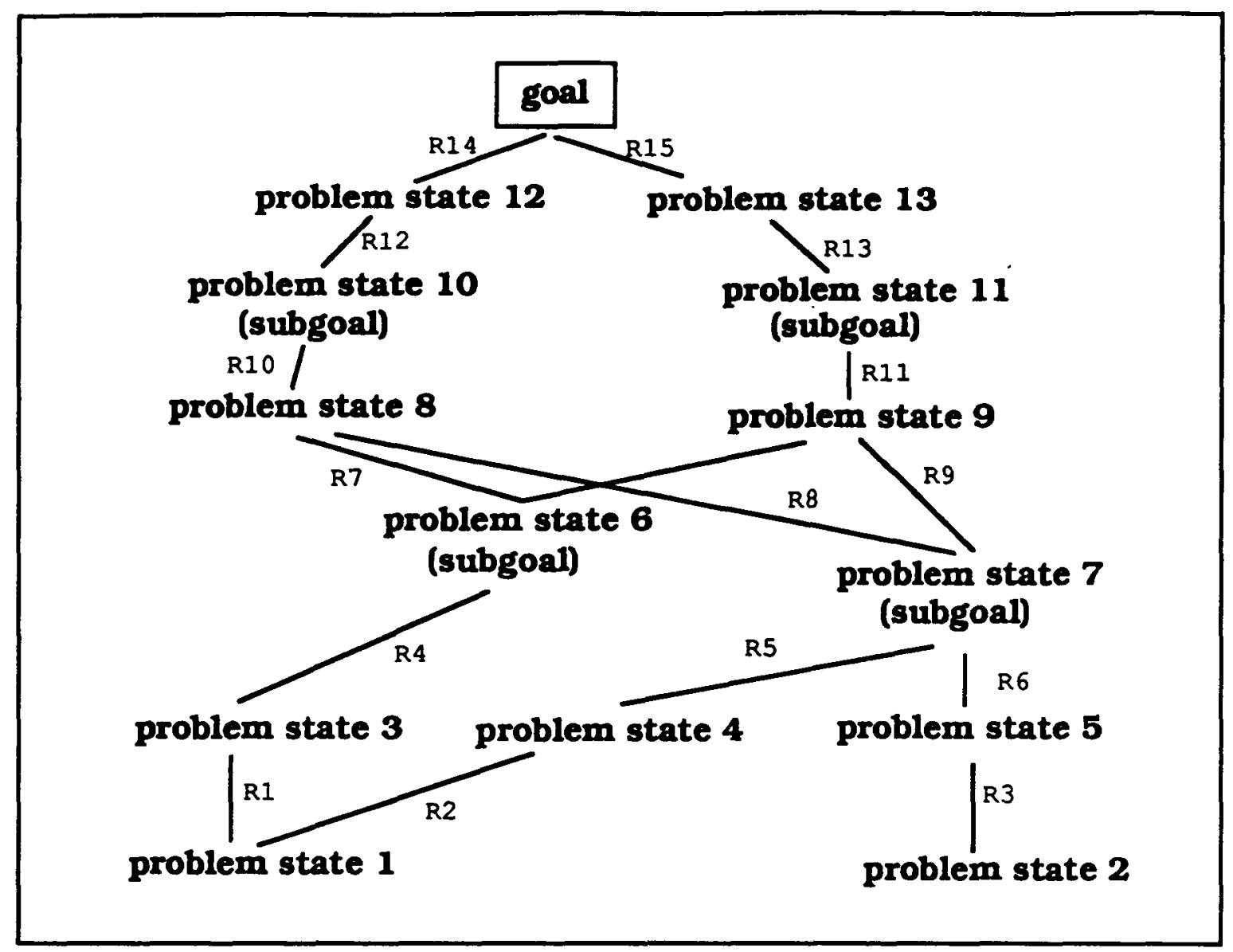

Cognitive Skill is also Defined by:

1. Knowledge of Task Goal and Subgoals

2. Strategles to Achieve Subgoals

Figure 2-2. Cognitive skill at simple task level of analysis. 
are the more efficient task-specific processes that have developed through training.

This framework also provides the concept of a mental "strategy," which is a means to guide the stringing together of individual rules to achieve some task-relevant goal. This reflects the metacognitive level of cognition. In electronics troubleshooting, skilled technicians use a "splithalf" strategy to isolate faults (Means \& Gott, 1988). For example, if there is a bad test point in a large, complex circuit, the technician first tests for a good signal halfway between that point and the input (which is known to be good). If that test is good, the fault lies downstream. If that test is bad, the fault lies upstream. By using this simple strategy iteratively, the technician can isolate a fault much more efficiently than by testing every point along the circuit. Thus, the technician is "managing" the application of rules.

Strategies are closely tied to an understanding of goals and subgoals. Obviously, it is often very difficult to achieve the ultimate goal with a single change in the current state. Typically, it is necessary to move toward a meaningful subgoal or to identify a string of subgoals that can be achieved in order to reach the goal (see Figure 2-2). Strategies are typically used to achieve a subgoal. In cases such as the troubleshooting example, the identification of subgoals is straightforward, but in other cases, the identification of useful subgoals is difficult. For example, in a study of Rubik's Cube, it was found that skilled solvers had developed strategies to achieve subgoals that appeared to be more different from the solution than where they started (Korff, 1987). That is, they used a string of individual moves to achieve a cube configuration that seemed to move them away from the solution, but actually gave them a configuration from which solution was possible.

Although this framework emphasizes rules and the stringing together of rules to achieve meaningful subgoals, the quality of the mental representation is also important. Research in cognitive psychology has shown that skilled performers use mental representations that better capture functional aspects of the task. Chess masters develop a mental representation of pieces on the chess board in terms of chess strategy (Chase \& Simon; 1973); physics professors mentally represent word problems in terms of basic principles of mechanics (Chi, Feltovich, \& Glaser, 1981). The extent of the representation can also be a factor. A skilled operator may begin with a fairly comprehensive mental model of the system that is being controlled. A less skilled observer may only have knowledge of simpler relationships between plant components without having the detailed functional knowledge. In addition, mental representations can differ in how well they support consideration of the system from multiple perspectives.

Thus, within this framework, cognitive skill becomes more broadly defined and more closely tied to realistic tasks. The critical elements of cognitive skill are the following:

- Task-relevant knowledge.

- Meaningful organizations of knowledge (mental representations).

- Task-relevant cognitive processes (rules) that can be used to modify the representation.

- The set of subgoals that provide efficient paths to the goal.

- Strategies (strings of rules) that can be used to achieve subgoals.

These are the critical elements of cognitive skills, and task analysis must identify them so they can become the focus of training. Traditional task analysis does not identify critical elements of cognitive skills. However, over the last 15 years, cognitive task analysis has been developed as a set of methods for identifying these elements of cognitive skill.

\subsubsection{Cognitive Skill in Complex Task Performance}

When considering performance in a larger context, such as controlling a nuclear power plant, performance is complicated in several ways. First, skilled operators require cognitive skills as well as perceptual skills, motor skills, and procedures. Many individual skills must be integrated to support performance of such complex tasks (see Figure 2-3). Further, performance at this level involves multiple individuals that must coordinate skills to achieve certain goals. With this integration comes an increased need for management of task performance and an even greater reliance on metacognitive skills. Mental resources and physical resources must now be 

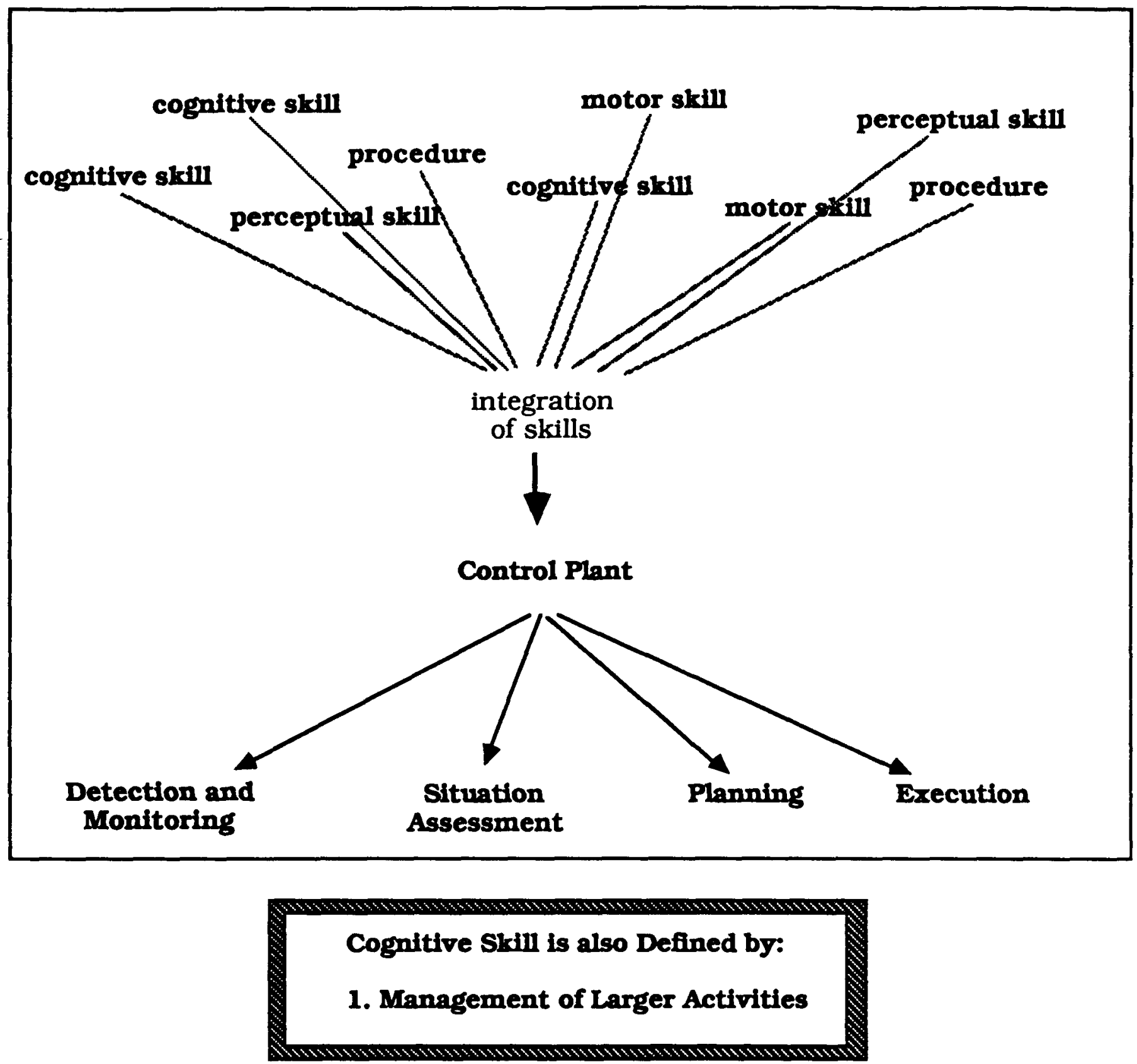

Figure 2-3. Cognitive skill at complex task level of analysis. 
managed to complete a large number of inter-related tasks.

Moreover, in order to analyze performance during complex tasks, analysts often create somewhat artificial stages in a task to capture more broadly defined cognitive activities. At the highest level, NPP control is usually split into at least four broad activities shown in Figure 2-3: detection and monitoring, situation assessment, planning, and execution. These are intended to capture cognitive skills at the highest level of description. Situation assessment is central to cognitive skill and primarily concerns the initial and on-going development of a mental representation that can capture critical information on plant state. This representation must change over time as the event progresses and as new information is reported. In situation assessment, expectations are generated about what phenomena will occur and what plant state indications will become relevant. Planning aids in determining the intent of control actions: What should I try to accomplish and what actions are required to accomplish it? Another important task that is generated from planning is monitoring that the plan is being achieved. Thus, the cognitive skills at this level strongly parallel the cognitive skills necessary for a simpler control task: develop a meaningful representation and determine how to achieve the task goal(s). However, the broader scope of the task (i.e., in terms of number of task elements) requires one to shift periodically from efforts to achieve the task goal to efforts to manage the task.

Hence, the broadly defined cognitive activities are artificial distinctions that allow an analyst to focus on selected aspects of performance of a complex task. Simpler, more directed tasks are performed within the context of these broader activities. However, it is insufficient to attempt to capture cognitive skills only at the level of the simpler tasks. The broader cognitive activities also have implications for the allocation of cognitive resources for management of the task. Specific skills may be required for a number of activities, such as monitoring how well a plan is progressing or determining how well the situation assessment accounts for all available data. These are explored further in our analysis of performance.

\subsubsection{Summary}

This section began with a simplified description of basic cognitive mechanisms that revealed several critical characteristics of cognition--e.g., the distinction between representation and processing, knowledge-driven vs data-driven processing, limitations on working memory and attention. Then, cognitive skills were described at three levels of analysis. At the lowest level, the description focused on the application of processes to knowledge and the way that processing becomes more efficient over time. The unit of performance in this case is a simple decision, computation, or judgment. When the unit of performance was expanded to address realistic tasks, such as controlling a parameter or solving a problem, the concepts of goals, subgoals, strategies, and problem states were introduced. This framework places cognitive skills in the service of achieving task goals and provides a role for metacognitive skills. Finally, when the unit of performance took into account a complex, almost unbounded task, such as controlling a nuclear power plant, it was found that the same types of cognitive skills are required on a larger scale, and there is an increased need for cognitive skills to manage task performance. In particular, broader activities, such as planning, that strongly require the management of cognitive resources were identified. Not only are performers trying to identify the appropriate actions to achieve a task goal, they are also monitoring task performance goals. Figures 2-1, 2-2, and 2-3 capture many of the important concepts.

\subsection{Related Concepts}

Based on the framework presented above for discussing cognitive skills, the following terms are defined: task, knowledge-based performance, rulebased performance, and decision making.

\subsubsection{Task}

An important distinction is made between a task and a skill. Tasks are described in terms of job functions and goals. Specifically, Sticha et al. (1990) define a task as a "job-specific goal that must be satisfied or a function that must be accomplished." Major job functions (e.g., increase power level, provide core cooling) are typically analyzed into tasks. These 
tasks are then organized, either sequentially or hierarchically, to reveal logical relationships between tasks and high-level job functions. A task description determines what has to be done and when to do it. Task descriptions do not identify the "how to do it" aspect, which is covered by the term skill. In this way, a task description does not rely on the specification of skills or the agent performing the task (e.g., man or machine). Thus, a task may be achieved through a variety of means.

\subsubsection{Knowledge-Based, Rule-Based, and Skill- Based}

Another important distinction in the area of skills is offered by Rasmussen (1986) through his terms skillbased, rule-based, and knowledge-based.

Rasmussen's terms are defined as follows:

Skill-based: performance that takes place without conscious control; smooth, automated, and highly integrated patterns of behavior. Simple examples can be taken from sports (catch a ball, swing a bat or racket) or driving (riding a bicycle, turning a car).

Rule-based: performance that is consciously controlled by a procedure or set of rules. Although individual behaviors may be skillbased, their performance is structured by a set of rules, which are often documented in a formal manner (e.g., recipe, procedure). The set of rules also captures the purpose or goal of performance. In NPP operations, the highly formalized procedures represent the results of a prior analysis of appropriate actions, and the goal of each set of actions is well documented.

Knowledge-based: in unfamiliar or unanalyzed situations, performance is generated from a reasoning process. Often, the goal of a task must be determined from an understanding of the situation, and individual behaviors must be identified out of an analysis of the goal. In NPP operations, the type of analysis that is used to develop formal procedures must be carried out on the spot (without procedures) to specify the appropriate actions.
Cognitive skills, as described above, are critical to knowledge-based performance, which relies heavily on extensive and detailed knowledge of systems and processes (mental models), an ability to determine the goal of the task, and an ability to identify the set of individual actions to achieve that goal. The nuclear power industry strives to remove the need for knowledge-based performance by analyzing likely events beforehand and developing detailed procedures for operators to use. When detailed procedures are available, operators can use rulebased performance. However, the full set of procedures being developed for SAM by the various owners' groups will not in all instances achieve the same level of detail as emergency procedures. Therefore, it may be possible to see cases for which knowledge-based performance is needed.

While it may be obvious that cognitive skills are required for the rare instances when procedures are insufficient and knowledge-based performance is demanded, cognitive skills may also be required for aspects of performance considered to be rule-based. NPP operators need to assess the appropriateness of an action to determine degree, timing, or even whether the action should be taken at all. The assessments that operators make, which are knowledge-intensive, typically rely on an evaluation of the intent of the procedure and the perceived state of the plant. Section 3.1 provides examples of the use of cognitive skills in procedure use.

Thus, Rasmussen's distinctions are not identical to distinctions between cognitive and non-cognitive skills. They reflect instead the degree to which cognitive skills may be required for the control of performance. Knowledge-based performance refers to situations in which little or no procedural support exists, and operators and technical staff must use knowledge and reasoning abilities to determine the most appropriate action. Cognitive skills are essential to knowledge-based performance. Rulebased performance refers to situations in which a formal, detailed procedure is available to determine the appropriate actions. Cognitive skills are required for rule-based performance to the extent that judgment or discretion may be needed to use the procedure. 


\subsubsection{Decision Making}

The term "decision making" is usually applied narrowly to refer to the act of selecting among several alternatives. Much of the early psychological literature on decision making (e.g., Tversky \& Kahneman, 1974) dealt with wellconstrained tasks in which decision makers were asked to evaluate a handful of choices and select the best--for example, selecting the best refrigerator. These studies focused on the way individuals sampled and weighed different types of information about the options and the types of information that influenced choices. More recent work by Klein (e.g., Klein, 1989) addresses more complex and dynamic tasks, such as command and control and fire fighting, but still reserves the term "decision making" for selection of a response from among alternative responses.

In this report, decision making is used more broadly to refer to the full set of activities required to select an appropriate action in an operational context. In the tradition of Rasmussen (1980), NPP operations are described as a series of decisions concerning the selection of appropriate control actions. Thus, decision making includes everything from the initial indication that action is required to actual execution of the selected action-activities both prior to and subsequent to the selection of an alternative. The primary reason for treating the term so broadly is the desire to identify the cognitive skills that may be important to performance in NPP operations, and especially in SAM, since cognitive skills can influence operator and technical staff performance in a variety of ways.

\subsection{SAT and Cognitive Skills}

Since its development, the Systems Approach to Training (SAT) has been the dominant force in guiding large instructional development programs. SAT provides a standardized structure to training design activities that ensures the development of a comprehensive, managed training program.

However, SAT is most easily applied to procedural tasks and is less typically used to support the development of effective instructional materiais for jobs that rely heavily on cognitive skills.
This is not to say the SAT cannot accommodate cognitive skills. The approach encourages a comprehensive task analysis that is followed by an analysis of the types of skills required to perform tasks, and cognitive skills are usually identified from this analysis. For example, in analyzing the tasks and subtasks that comprise a job, the training developer typically finds a need to use verbs such as "decide" and "determine"--for example, "determine when the x procedure needs to be applied." Job incumbents will verify that this determination must be made and that trainees must learn to make the determination. Further, the task analyst will correctly label this as a cognitive skill. However, the task analysis often stops at that point because techniques are not available for more detailed analysis of cognitive skills. That is, SAT, by itself, does not provide techniques to identify the elements of cognitive skill as laid out here.

An illustration of the lack of a detailed cognitive task analysis was found in a review of the Federal Aviation Administration's air traffic controller training program (Means et al., 1988). The cognitive skill that is the essence of air traffic control is determining when a potential conflict exists--what controllers call "seeing the traffic." Standard task analysis had identified this cognitive skill as a critical component of the job years earlier, but an analysis of the critical elements of that skill had not been conducted and, therefore, had not translated that insight into effective training. After the training-program review, an application of cognitive task analysis to this cognitive skill allowed Means et al. to begin to identify the strategies that skilled controllers used as well as important characteristics of their mental representations.

In this report, techniques and methods are described that have been developed to analyze cognitive skills and supplement SAT. With these techniques, cognitive skills can be analyzed into components that can be used to structure training. The knowledge, knowledge organization, cognitive processes, and strategies required for skilled performance can be identified and then established as goals for training. 


\section{Chapter 3 \\ Complex Decision-Making Tasks in Nuclear Power Plants}

Decision making was described in Section 2.2.3 as the full set of activities required to select an appropriate action in an operational context. This broad definition of decision making includes everything from the initial indication that action is required through actual execution of the selected action, and eventually, acting on the resulting plant response. For the vast majority of cases in NPP operations, detailed procedures exist to guide the selection of actions, and decision making is predominantly rulebased. Indeed, current procedures inform operators about which indications to monitor, the assumptions to make, the goals to strive for, and details of executing the control actions. However, even when detailed procedures exist, this decision-making task can become complex and require cognitive skills. Section 3.1 describes the types of situations and factors that increase the complexity of decision making when procedures exist. This section also provides evidence that operators do not rely on procedures alone to guide performance--that is, cognitive skills are used to supplement the procedures in determining appropriate behaviors. Section 3.2 describes decision making as it is likely to be carried out during a severe accident. The difficulty and complexity of operational decision making in this case may increase by an order of magnitude when compared to more standard control tasks.

\subsection{Cognitive Skills in Decision Making During Routine and Emergency Operations}

Detailed procedures exist to guide operator decision making for the vast majority of control actions. Utilities upgrade, refine, and validate these procedures periodically to maintain them at a high level of accuracy and completeness. However, there is growing evidence that procedures alone may not provide all that is needed. First, there have been a number of reports initiated by investigations of NPP incidents (Haber, 1989; Kauffman et al., 1992) that suggest that operating procedures do not always provide adequate guidance to operators. The two primary reasons given are that: 1) procedures may contain insufficiently detailed guidance for certain control actions, and 2) it is very difficult to control and update procedures sufficiently to remove all errors. For example, a report from the NRC's AEOD (Kauffman et al., 1992) provides an example in which an NPP's procedures assumed a component was in a start-up state when it was not.

Another concern is that certain types of failure scenarios are not addressed well by procedures because these scenarios present a type of complexity that is not considered in procedure design. Mumaw and Roth (1992) have catalogued some of the situations that create these complexities, including:

- Situations that remove or obscure the usual evidence or critical evidence. For example, in certain PWR designs, a loss of off-site power prior to a steam generator tube rupture can isolate steam generator effluents and eliminate the initial indications (i.e., radiation sensors) of the tube rupture.

- Situations in which important indications can be incorrectly "rationalized away." That is, indications that should be diagnostic may be attributed to a consequence of a known but less significant failure or of an automatic action (e.g., shrink and swell).

- Situations in which multiple faults mask each other.

- Situations in which faults produce effects at a distance. For example, in some interfacing system loss of coolant accidents, indications can appear in multiple systems that are not commonly associated.

- Situations that deviate from operator assumptions or expectations. For example, a safety system may fail to respond as demanded, or human errors occur in carrying out control actions to mitigate a component or system failure. 
These are the types of situations that have contributed to nuclear power plant incidents, and because of the complexity it is difficult to modify procedure design to improve operator performance in these situations. Major changes occurred in procedure design after TMI-2, and procedures are vastly improved over earlier forms. The primary improvement was the shift from diagnostic to symptom-based procedures. Diagnostic procedures required operators to understand the current plant state (i.e., faults, system failures) and select the procedure most appropriate to that state. Symptombased procedures, on the other hand, require operators to monitor a handful of critical safety functions. When one of these functions is violated (e.g., core cooling), the operators select a procedure to restore that function. Thus, these procedures help operators prevent further deterioration and achieve a safe and stable state while seeking the cause of the event. However, studies have shown that even the shift to symptom-based procedures does not guarantee effective and efficient resolution of all plant faults.

Some investigators have recommended that operators or other technical staff rely not on procedures alone, but also on their own reasoning about the state of the plant and the set of control actions that are most appropriate. That is, some believe there is a legitimate need for operators to develop cognitive skills that supplement procedures to provide a diverse, redundant monitoring capability. Others argue that doing so introduces an unknown element to NPP control.

This issue-namely, the necessity of training cognitive skills-is a difficult issue to resolve. However, the resolution of this issue may be informed by investigations of operator performance during difficult situations. For example, recent studies provide evidence that operators currently use cognitive skills to supplement the guidance provided by the procedures. The following paragraphs describe this evidence.

Recently, the U.S. Nuclear Regulatory Commission has supported a program to model the cognitive activities that underlie operator intention formation during NPP emergencies (see e.g., Roth, Woods, \& Pople, 1992). The emphasis of this research program is to try to understand and model the cognitive processes of operators as they are trying to handle challenging accident situations. This program has involved two inter-related activities: 1) analyses of crew performance during simulated emergencies, and 2) development of a computer-based model, called Cognitive Environment Simulation (CES), that simulates some of the cognitive activities involved in responding to an NPP accident situation. The process of building CES to handle the events and then comparing its performance to the performance of the crews on the same events has enabled researchers to gain insight into:

- The cognitive demands imposed by challenging NPP scenarios.

- The knowledge and reasoning capabilities required for successful performance on the scenario.

- The knowledge and information processing limitations of crews that restrict their performance.

More important for the current context, the analysis of crew performance has revealed cases in which operators supplemented the guidance provided by the procedures with guidance derived from their own reasoning about the plant. More specifically, to increase their efficiency, crews either took actions prior to the time the procedure would require them or took actions not found in the procedures. The following paragraphs provide examples from these analyses that demonstrate the value of operators' cognitive skills.

Actual crews were observed executing two scenarios presented in a PWR training simulator. One of these events was an interfacing system loss of coolant accident (ISLOCA). In this incident, a failed open valve was simulated between the reactor coolant system (RCS), which is inside containment, and the residual heat removal system (RHR), which is outside containment. In the ISLOCA simulation, the flow of the RCS coolant into the RHR led to an increase in pressure in the RHR and, eventually, a break in the RHR piping. Because RHR relief valves lead to the pressurizer relief tank (PRT), the simulation introduced the opening of the PRT rupture disk and the release of steam and radiation to containment. This event can be challenging because it produces symptoms in multiple regions of the plant that are not commonly associated. Thus, the abnormal radiation in containment and in the RHR 
may mislead operators to believe there are multiple, independent breaks.

There were several indications that operators used reasoning to supplement the use of procedures. First, operators attempted to develop a coherent explanation of the symptoms observed. For example, because of a failure to observe the symptoms expected from a LOCA inside containment, several of the crews recognized early on that it was not a straightforward LOCA and began suspecting and seeking some other (additional or alternative) leak outside containment. Other crews also showed evidence of active search for a coherent explanation of the diverse symptoms presented by the scenario. Although faced with symptoms that did not at first seem compatible, some crews continued to seek explanations that minimized the number of separate faults needed to account for the symptoms.

Eventually, these crews were able to come up with a coherent causal sequence that simultaneously explained all the symptoms.

A second indication that crews were supplementing the procedures with their own reasoning were signs of anticipation. For example, at the point an ISLOCA was suspected, several of the crews immediately called down to the auxiliary building to search for evidence of a possible leak outside of containment, a step that appears late in the procedure. By anticipating this step, operators were able to initiate the call early so that they would have the results of the auxiliary building search already available at the point where the procedure called for it. In another instance, operators, cued by the PRT rupture, were able to identify the problem in the RHR before they reached a point in the procedures that explicitly asked them to check for RHR symptoms.

There was also evidence in this study that operators periodically assess procedures according to their understanding of the goals and strategies that define the procedure's intent. In one case involving steam generator pressure, it was found that crews executed a branch in the procedure that was incorrect from a "letter of the law" perspective. Specifically, although steam generator pressure was found to be decreasing, the crew branched in the procedure as though it was stable because they understood the intent of the procedure at that point. Other cases were observed where operators, based on their understanding of plant state, concluded that they were in the wrong procedure for the current situation and, because of this, actively switched to a procedure they believed to be more appropriate. In particular, one crew had correctly followed the procedures and branched to a LOCA-specific procedure. Later, because their understanding of the situation evolved, they decided that the ISLOCA procedures contained relevant guidance and consulted that procedure. Note that this crew did not violate procedural guidance; they continued on through the LOCA procedure. However, when they reached a hold point in that procedure, they accessed and performed the ISLOCA procedure in parallel.

Thus, this study showed that operating crews engage in cognitive activities and use them to supplement the procedures in determining many aspects of performance (see also, Kauffman et al., 1992). In particular, crews monitor indications and symptoms, anticipate and watch for the occurrence of larger phenomena, interpret procedural criteria, and execute procedural branching points in ways that suggest an understanding beyond that provided by a simple reading of the procedures. Thus, there seems to be clear evidence that cognitive skills are important when performance is rule-based.

Tying these observations back to the discussion of cognitive skills in Chapter 2, one might conclude that crews are engaged in situation assessment, which allows them to identify relevant parameters and indications and allows them to anticipate and monitor related phenomena. Also derived from the situation assessment, when linked to knowledge of the procedures, is a clear understanding of the goals and subgoals that need to be achieved (i.e., the general issue of planning). More peripheral are cognitive aspects of selecting and executing control actions. For example, potential undesirable side effects of control actions can be assessed within the mental representation before an action is actually taken.

The next section discusses complications in the decision-making process that may arise during severe accident management. These additional difficulties may create even greater requirements for cognitive skills. 


\subsection{The Special Case of Severe Accident Management}

Although the processes for decision making during SAM have not been completely specified by every owners' group, initial drafts make it clear that SAM decision making may differ significantly from the types of decision making common to NPP operations. Each owners' group-Combustion Engineering, Babcock \& Wilcox, Westinghouse, and General Electric-has completed or will soon complete a draft decision-making process that represents a generic version of the severe accident management guidance (SAMG) document. This document is similar in function to the emergency response guidelines (ERGs) in that it specifies a plan for response and a justification. The utilities will then develop plantspecific versions of the SAMG. Although this process is not complete at this time, many of the general characteristics of the SAM decision process are known. This section describes five likely differences between the SAM decision-making process and emergency operations. However, because the four owners' groups are not taking an identical approach to SAMG development, not all of these differences will apply to each.

\section{Decision-making authority may be shared or} transferred by the control room and Technical Support Center. In current procedures, the control room (CR) staff are primarily responsible for all decisions and actions. In a few cases, the EOPs direct the CR staff to consult the Technical Support Center (TSC) for guidance. SAM, however, is likely to give a much larger role to the TSC. In some cases, decision-making and/or control will be passed from the CR to the TSC and then, perhaps, passed back again. Most utilities allow at least one hour to make the TSC ready to become involved in decision making.

One possible scenario, then, is as follows. Initially, as the event progresses from one handled well by emergency procedures to one that perhaps cannot be addressed by emergency procedures, the $C R$ maintains authority and uses available procedures to the best of their ability. During this time, the TSC is being staffed and brought up-to-date on the event's progression. At some point, perhaps marked by some observable criteria (e.g., crossing a parameter boundary, time elapsed) or by someone's judgment, decision-making authority will be given to a member of the staff and may be accompanied by a transition into the SAMG. The SAMG will support the TSC in identifying the most appropriate course of action. However, to execute control actions, the CR staff must reclaim some level of control. Again; this is just one possible scenario, but it is likely that SAM will involve the sharing or transfer of decision making.

This situation introduces some potential difficulties into the decision-making task. The TSC will be less familiar with the event's progression and with the current set of transitory control room peculiarities (e.g., tag-outs, reliability problems). The TSC may also have less access to plant state data, both historical and current. The TSC's access to plant state data varies from plant to plant, but in general, there are likely to be some restrictions.

Another difficulty with the transfer of decision making to the TSC is that the CR will ultimately be required to carry out the recommended control actions. If the $C R$ is not involved in the decisionmaking process, they may not know the assumptions that have been made about plant status, they may not know which alternatives have been eliminated, and they may not understand the intent of the procedures handed to them by the TSC. These types of information can be critical to executing control actions efficiently and adaptively. For example, equipment and system status can change, and the $C R$ staff need to understand how to adapt to changes in the plan.

In summary, SAM decision making is likely to involve critical inputs from two groups in different locations; each group will have different perspectives, different knowledge of the plant and the event, and perhaps, different decision guidance documents. The authority for making decisions, taking control actions, and judging success may be distributed across these two groups. These conditions call for excellent communication and group problemsolving skills to integrate all sources of information.

\section{Reliable and accurate plant state data will be} more difficult to obtain. There have been a number of reported incidents (e.g., Kauffman et al., 1992) in which plant sensors have failed or in which operators must determine the validity of an alarm. A severe accident setting may make these occurrences 
more likely. In order to progress to a level of severe accident, there are likely to be multiple failures of equipment, safety systems, and humans. Under these conditions, accurate and reliable indications of plant state may become more difficult to obtain. First, current plants are not instrumented to provide detailed information on core status during challenges to core cooling. Second, some indications may be totally lost as systems fail or as equipment availability is lost due to flooding or contamination. Third, some plant sensors are reliable only within certain environmental constraints. Even when an indicator appears to be functioning under hightemperature conditions, operators and technical staff may not be able to rely on the indication.

The problems in obtaining accurate and reliable data in the CR may be amplified in the TSC. Although plants vary widely in their computerization of the TSC, some TSCs are more likely to have access only to processed or integrated data displays and may, therefore, have less access to data reliability information.

Because decision making relies on an understanding of plant state (or at least critical function status), and because the appropriateness of control actions may depend on plant state, decision making is complicated by the reduced availability of reliable and accurate plant state data during a severe accident. Skilled decision making, therefore, may have a greater dependency on deliberate efforts to ensure the most reliable data possible.

\section{The SAMG will not be prescriptive to the same} degree as current emergency procedures. Judging from the initial drafts of the SAMG submitted by three of the four owners' groups, it appears that, in general, the SAMG will not specify control actions at the level of detail common to the EOPs. First, there will be less diagnostic information regarding the progression of core or vessel damage. The location of the core and its status cannot be determined directly, and therefore, the decision maker may be unable to determine the set of phenomena that must be addressed.

Second, it may be difficult to implement actions to reduce the threat from one challenge without increasing the risks from other potential challenges. Sometimes one goal will be directly compromised through actions to resolve a second goal (e.g., recriticality may be affected by core cooling). In other cases, an action may only eliminate a set of control actions that are not currently needed but may be needed at a later time (e.g., flooding that covers equipment, venting that contaminates service areas). In addition, because of the severely degraded state of the plant, there may be more than one serious challenge present at any one time.

Third, the appropriateness of a set of control actions may rely on having certain equipment available, having sufficient time to start up or re-align systems, or on determining how to reduce potentially severe side effects. These uncertainties make it difficult for the SAMG to specify precisely the optimal response to any challenge, and because of this level of uncertainty, the selection of the most appropriate response may have to be made on the spot. The SAMG may only offer a set of alternatives from which the decision maker must choose to ensure an optimal response. Thus, the extent to which procedure users need to assess the appropriateness of a given mitigation strategy has increased significantly.

4. Uncertainty in decision making will be increased. A number of the issues already identified reveal the higher levels of uncertainty inherent in SAM. In general, decision makers will be asked to select and carry out control actions that have potentially severe consequences in an environment where information is difficult to obtain and phenomena are not well understood. There may be less information about plant status ("Maybe if I wait longer I will know the state of $\left.x^{\prime \prime}\right)$. There may be less opportunity to implement the preferred course of action ("Maybe if I wait longer I will be able to recover and use system $\left.x^{\prime \prime}\right)$. There is reluctance in any situation to take actions that have known severe consequences ("Maybe if I wait longer I won't have to flood x"). In addition to these sources of uncertainty, a severe accident will quickly attract interested parties from utility, community, state, and national organizations.

5. Stress levels on decision makers will be increased. Any emergency situation introduces stress to operators and technical staff. However, there are a number of reasons that SAM will involve stress levels exceeding those expected from design-basis accidents. First, one primary source of stress is novelty or uncertainty. Research shows that stress 
levels are high when decision makers have few or no expectations about the likely progression of an event. Second, high workload and perceived time pressure increase stress levels in performers. Third, the significance of the event and the heightened attention to every aspect of performance can create high levels of performance anxiety. Finally, there is a chance that the physical environment of the plant could become adverse (e.g., high temperatures, threat of radiation) and require protective clothing, which is cumbersome. These factors, also, can increase stress levels for performers. Because high stress levels can impair decision-making performance, SAM decision making must have higher demands for skilled performance. (See the companion report, NUREG/CR-6127, for a more complete review of sources of stress.).

Summary and conclusions. The issues described here represent difficulties that have the potential to be introduced to decision making during SAM. In combination, they make SAM decision making stand out as a special case of decision making that has characteristics not found in emergency operations. Moreover, these issues suggest the potential for an increased reliance on cognitive skills. The types of cognitive skills that are likely to become important are identified in the next two chapters. 


\section{Chapter 4 \\ A Model and Analysis of NPP Decision Making}

A primary objective of this report is to describe the types of cognitive skills that are required for NPP decision making. In this chapter, more detailed analysis of NPP decision making, in the form of a performance model, is provided as one means for identifying necessary cognitive skills.

\subsection{Overview of Analytic Approach}

The ideal approach for identifying specific cognitive skills associated with NPP decision making, and in particular, SAM decision making is to conduct a cognitive task analysis. The standard progression of task analysis is shown in Figure 4-1. Initially, the complete set of job functions is identified. Each job function is then analyzed into the set of tasks that are required to achieve the function. Tasks may be analyzed further into subtasks until the analyst has a complete and detailed set of task descriptions. During this process, the analyst may also rate each task on the following dimensions:

- Criticality - How critical is the task to achieving the job function or to overall plant safety?

- Difficulty - How difficult is the task to learn and to perform in a skilled way?

- Frequency - How frequently will each task be performed under standard operating conditions?

The next step in task analysis is to identify and classify the knowledge, skills, and abilities (KSAs) that are required to perform each task. This type of analysis typically identifies entities such as basic or theoretical knowledge of a process or system (e.g., basic thermodynamics), equipment operation skills, critical decisions, perceptual distinctions, etc. These are then classified into categories such as cognitive skills, perceptual skills, manual skills, procedures, etc. for purposes of designing training.

In order to describe cognitive skills in more detail and with greater precision, a cognitive task analysis is used to supplement the standard task analysis.

Cognitive task analysis can be used to determine the mental strategies and characteristics of mental representation that are essential to skilled performance, or it might focus on analyses of critical decisions (e.g., information requirements, goal structures). These methods have been employed and refined in previous analyses (Lesgold et al., 1986; Means et al., 1988; Roth \& Woods, 1988). Roth and Mumaw (in press) provide a brief overview of cognitive task analysis techniques.

Thus, this process is one avenue to identifying cognitive skills. However, performing these extensive task analyses requires starting with a well-defined job and/or access to practitioners of that job. In the case of SAM decision making, the job is not yet well defined-that is, job functions or task-subtask hierarchies have not yet been developed. Therefore, a more analytic (i.e., less empirical) approach to identifying the cognitive skills required for NPP decision making was developed.

This approach, illustrated in Figure 4-2, used as a starting point a model of decision making, borrowed from Rasmussen (1986), that has been widely applied to NPP operations. This model was modified to capture performance in both standard procedureguided (primarily rule-based) decision making (normal and emergency operations) and SAM decision making, which has a greater likelihood to be knowledge-based.

The model was used to identify cognitive skills. This was accomplished by applying two perspectives on performance to the model. The first perspective brought to bear analyses of skilled performance. In other words, what are the cognitive skills required to perform NPP decision-making tasks? If a model of skilled performance can be defined, it provides a set of cognitive skills that can serve as the goals of training. Applying this "skilled performance" perspective relied partially on analyses of expertise in strongly related tasks (e.g., problem solving, troubleshooting, decision making in non-NPP settings) and partially on previous analyses of NPP operator decision making conducted for other purposes (e.g., display design, control room design). Thus, because cognitive skill requirements for similar tasks are well understood, one can apply that 


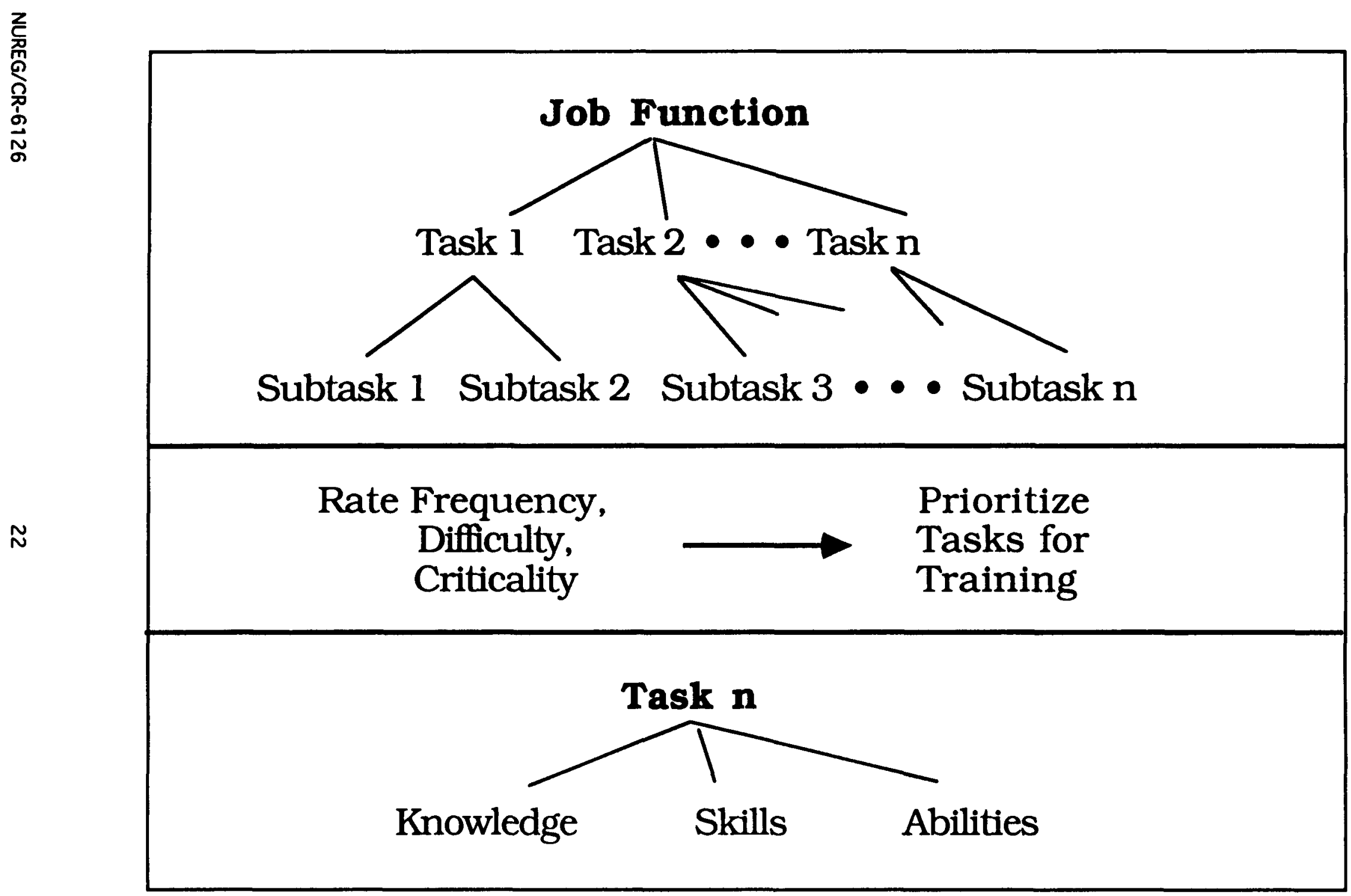

Figure 4-1. Standard approach to task analysis. 
Training Goals:

Description of

Skilled Performance

(Rule-Based and

Knowledge-Based)

$\tilde{\omega}$

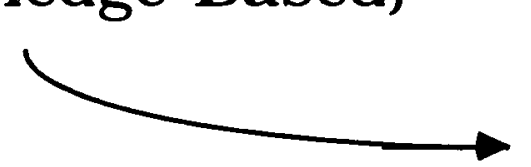

Training Needs:

- Cognitive Limitations

- Decision-Making Biases

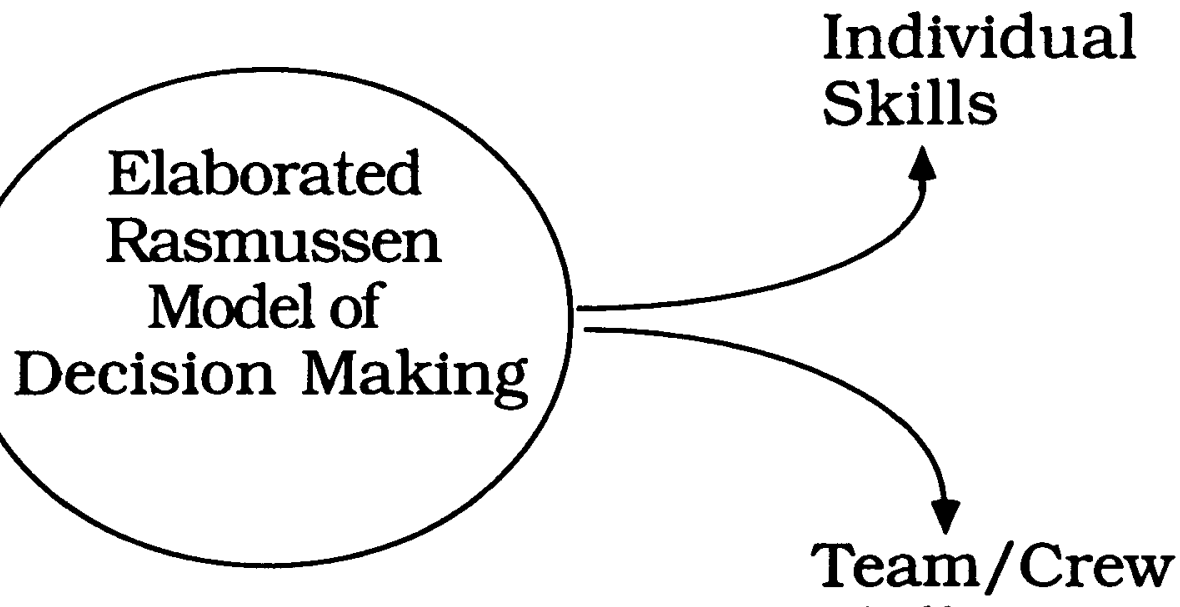

- Effects of Stress

Figure 4-2. Schematic of the analytic approach to the identification of cognitive skills. 
knowledge to the specifics of decision making in the current context.

The second perspective applied to the model was a consideration of the factors that can impair skilled performance. The limitations and biases inherent in human cognition and the effects of the stress associated with the NPP setting have the potential to impair performance in NPP decision making. Therefore, training may need to focus on cognitive skills that mitigate these factors, or training may be needed to make these cognitive skills less susceptible to the deleterious effects of these factors.

As Figure 4-2 shows, these two perspectives were applied to the model of decision making to identify cognitive skills. Because NPP decision making, especially SAM decision making, occurs in the context of a crew, the skills were further classified into individual skills and crew skills. Most cognitive skill training is directed at individuals, and training at the crew or team level has only recently been considered. However, it was critical to address both levels for this analysis.

The key points in summarizing our approach are the following:

- An empirical task analysis (either within the standard framework or the cognitive framework) was not possible for this program.

- An accepted model of NPP decision making was identified and adapted.

- The cognitive skills required for skilled performance were considered, and the cognitive skills that may be required to mitigate factors that can impair performance were considered.

- Crew skills were identified in addition to individual skills.

The next section describes the decision-making model in more detail.

\subsection{Model of Operator and Technical Staff Decision Making}

This section first describes the focus and nature of our performance model and then provides an overview of its components.

\subsubsection{Performance as Decision Making}

Before exploring the details of the performance model, it is important to comment on what is meant by the term "decision making." For the purposes of this project, all SAM activities have been reduced to a single activity, broadly defined as decision making. This was done because, first, the focus of this project is on the cognitive skills associated with SAM. The second point is that decision making in this context is defined to encompass many activities both prior and subsequent to the actual "selection of an alternative" (the narrow definition of decision making that perhaps first comes to mind). In the tradition of Rasmussen (1980), operations are described as a series of decisions concerning the selection of appropriate control actions. Therefore, because decision making is defined broadly, the model also includes supporting activities such as data gathering.

A note about the function of this decision making model: There have been numerous attempts to develop computer-based simulations of cognitive performance, both generally (Anderson, 1983; Newell, 1990) and for nuclear power plant settings specifically (Roth, Woods, \& Pople, 1992). The purpose of those projects was to simulate accurately the process and products, and sometimes the time-course, of decisionmaking and memory tasks. When a model is developed that is shown to be a relatively accurate simulation of human cognition, the modelers can then produce human-like performance in novel situations. This is an invaluable tool for exploring theories of cognition, predicting human errors, etc. However, these models are currently limited by the scope of the tasks that can be modeled; typically the knowledge that is possessed by the simulation is severely limited when compared to a human.

Modeling for the purpose of task analysis is much simpler, and the performance model presented here is not intended to be a detailed cognitive process model that could be converted to a computer simulation of decision making. Instead, it is intended to be a representation of the critical decision-making processes required by nuclear power plant operators and technical support personnel. The identification of these processes allows one to bring to bear what cognitive psychologists have discovered about each process so that the knowledge and skills that may be required for skilled performance and the sources of 
error that are likely in an SAM setting can be identified.

Finally, before exploring the details of our performance model, it is important to recall the distinction between two forms of decision making: rule-based and knowledge-based. Rule-based decision making refers to decision making that is controlled by a procedure or set or rules. The procedure also captures the purpose or goal of performance. In NPP operations, the highly formalized procedures that are used represent the results of a prior analysis of appropriate actions, and the goal of each set of actions is well documented. Knowledge-based decision making refers to cases in which no procedure exists or in which the procedure is insufficient to guide operations personnel. Often, the goal of a task must be determined from an understanding of the situation, and individual behaviors must be identified out of an analysis of the goal. In other words, operations or technical support staff must reason on their own to determine the best course of action. To do so, they rely on their knowledge of the plant, knowledge of related procedures, knowledge of safety systems, etc. Obviously, the cognitive skills required for knowledge-based decision making are more complex than those required for scripted decision making.

The performance model captures both rule-based and knowledge-based decision making since, during the course of a transient that evolves into a severe accident, the SAM team may begin in the EOPs and then move either to an SAMG document or to a situation not sufficiently covered in either. The cognitive skills associated with both types of decision making were considered.

\subsubsection{Overview of the Performance Model}

Rasmussen (1980) developed a model of operator decision making that has become a standard of the industry. The model is shown in Figure 4-3. Flow through the model begins at the lower left box ("detection of need for action") and continues up the pyramid and back down, finally reaching "execute." This represents a single "decision" cycle, and the full cycle represents knowledge-based decision making. The lighter arrows that cut across the pyramid show typical shortcuts in operator decision making that account for rule-based and skill-based decision making. Rasmussen's model describes performance at a fairly high level, and others have elaborated on or adapted this model while retaining the basic structure (e.g., Woods, 1982; Pew, Miller, \& Feeher, 1981; Meyer, 1991; Mumaw, Roth, \& Stubler, 1991). This model has been adapted for this project. Figure 4-4 shows the six general processes Rasmussen and others have used for describing decision-making performance:

- Monitor / Detect - active (monitor) and passive (detect) means for acquiring data about plant state. Sources of data are alarms, indicators, CRT displays, other individuals, etc.

- Interpret Current State - the development of a mental representation of plant status. This representation may include interpretations concerning faults and causes of abnormal symptoms, or it may be only a prioritized list of symptoms (e.g., safety violations).

- Determine Implications - the determination of how the current plant state will progress (e.g., potential consequences, side effects). Also, a set of goals is defined in which more important goals are given higher priority and complex goals may be broken down into subgoals.

- Plan - the selection of a response plan, which could be a high-level action or formal procedure, that addresses the goal(s) with the highest priority.

- Control - the coordination and execution of a specific sequence of control actions.

- Feedback - the information gained from control actions is used to update understanding.

Figures 4-5 $(a-f)$ show how this basic model has been expanded to emphasize and make explicit certain aspects of performance. The following paragraphs describe the reasons for expanding each of the six general processes:

Monitor / Detect. Figure 4-5a shows that this process, which is used to acquire plant state data, has been expanded to three components. This expansion maintains a distinction between active and passive means for acquiring plant state data. Often, the first indications of an abnormal plant state are acquired through passive means (e.g., control room alarm). This is passive in the sense that the operator is not actively seeking that indication. Through alarms and other salient indicators (e.g., lights), the operator is often able to detect abnormal conditions. After operators have been alerted that an abnormal 


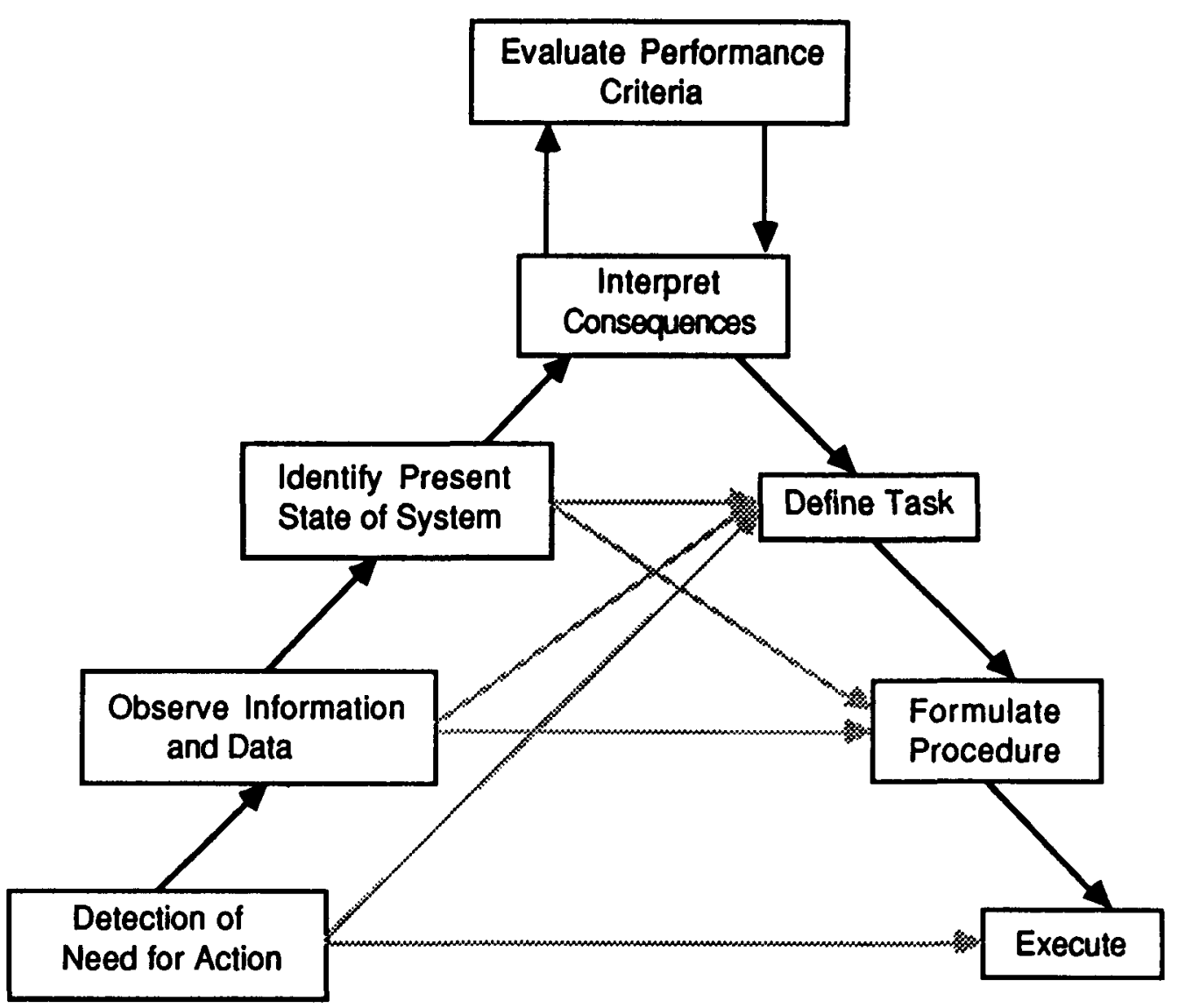

Stereotypical Shortcuts

Figure 4-3. Rasmussen's model of decision making 


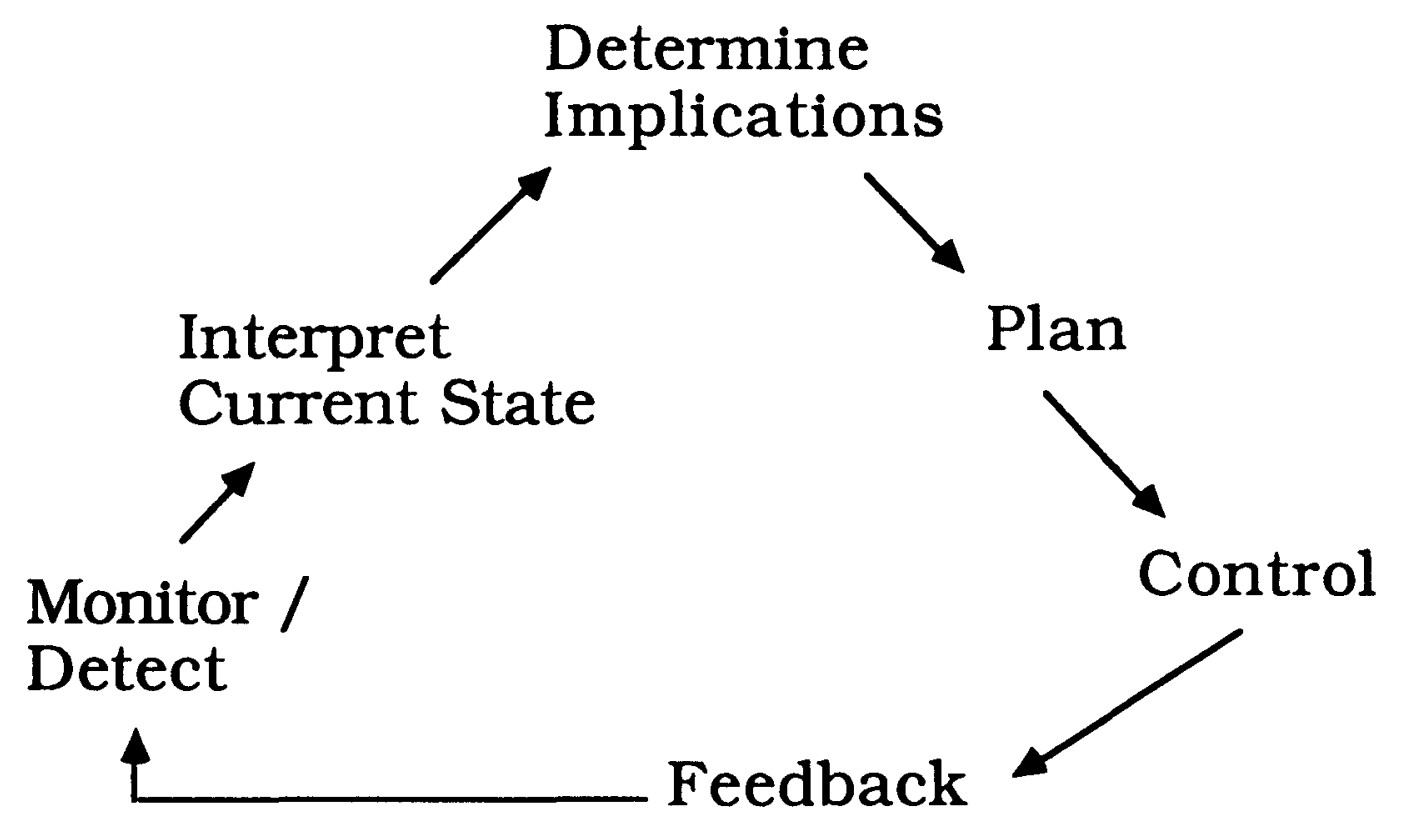

Figure 4-4. The six general components of the performance model 


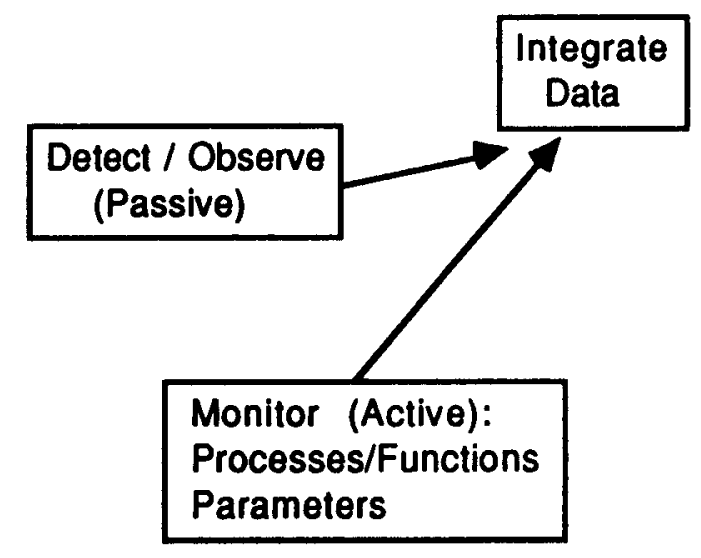

Monitor / Detect

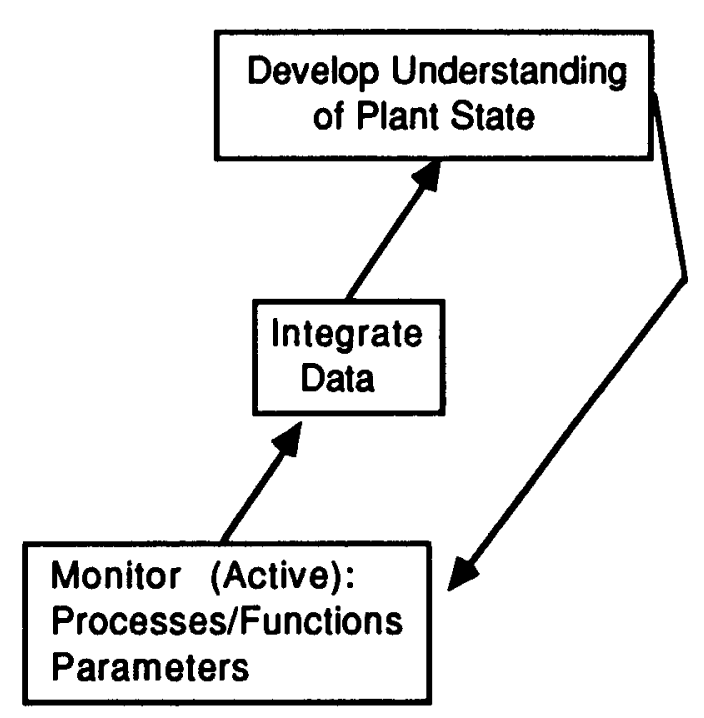

Interpret Current State

\section{Figures 4-5a and 4-5b}

Expanded versions of the six general performance model components 
condition may exist, they will acquire plant state data in a more active manner. At this point operators seek out specific indications, monitoring parameter values or monitoring indications that provide a description of higher-level processes or functions. As the transient evolves operators maintain both means for acquiring data.

The expansion of this process also address the need to integrate individual indications into a more complete picture of plant state. An issue is that, as the transient evolves, the TSC and other personnel outside the control room begin to take the major role in decision making. The access of TSC staff to control room data may be limited in several ways: there can be limits on the number of indications available at any time and there can be limits in the context provided with the indication. The number of indications is very likely to be limited because of the limited means for acquiring dynamic data in the TSC. The TSC can typically receive plant data through a $C R T$, which limits the number of parameters that can be displayed simultaneously, or through voice communications with the control room. Data context may be impoverished because the means for displaying data in the TSC do not retain important parameter setpoints or indications of scale range. In the worst case, a parameter that is displayed on a scaled meter in the control room is reduced to a simple digital readout on a CRT in the TSC. Information may also be lost in this way concerning data reliability.

Because of the issue of data availability in the TSC, the process of integrating plant state data has been made explicit. This integration can occur over time, when there are time delays in receiving strongly related data points. Or, the integration can occur in space in cases where there is a need to view the configuration of a number of parameters (e.g., a system mimic as an organization of individual indications).

Interpret Current State. The focus of this process, illustrated in 4-5b, is the decision maker's development of a mental representation about the current state of the plant. This may include information about existing faults or damage, equipment that is unavailable, and any abnormal conditions that exist in any plant system. A simpler representation may include only information about which critical safety functions have been violated since this level of knowledge is sufficient for the use of symptom-based EOPs.
This process has been expanded to emphasize the active nature of cognitive processing when developing a mental representation. The decision maker does not simply attempt to make sense of the plant state indications presented. Instead, he actively selects, organizes, and integrates certain indications that he believes will reveal some truth about plant state. Therefore, there is a loop including "developing understanding of plant state," "monitor parameters," and "integrate data." By cycling through this loop, the operator continues to update and refine his understanding of the plant state.

Determine Implications. As stated previously, this process is primarily concerned with the determination of how the current plant state will progress: What are the likely consequences?, What are likely side effects?, Which systems may become unavailable?, and ultimately, What actions need to be taken first? Figure 4-5c shows that there has been no increase in the number of process components devoted to the "determine implications" process. However, the notion "establish goal hierarchy" has been explicitly made a critical component of this process. The model assumes that decision making is tied to an analysis of goals (see Newell \& Simon, 1972). Thus, the outcome of this process must be some description of goal priorities. One possible description is a goal hierarchy, which places the most important goal at the top and less important goals at lower levels of importance. Goals may also be broken down into subgoals (e.g., recover use of a safety system) that are required to achieve the highest-level goal.

At this point goal trade-offs need to be considered. In some evolutions, several critical goals may be identified. The decision maker needs to determine whether these goals can be satisfied simultaneously or whether the goals are linked in some way. For example, actions to achieve one goal may place a second goal in conflict or delay action on the second goal. The need to consider goal priorities and goal trade-offs was an important element of the SAM scenarios and is an important issue in knowledgebased decision making.

Plan. The next step for the decision maker is to identify an approach for achieving the goal identified in the previous step. Figure 4-5d shows that this process has been expanded to account for the three potential paths for developing a plan. The first two paths rely on established plans in the form of written 


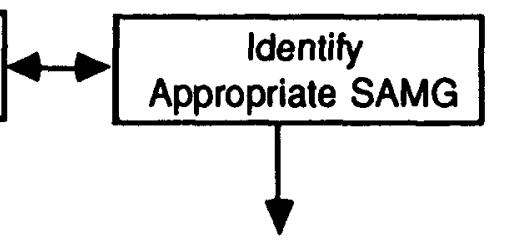

Determine Most Appropriate HLA

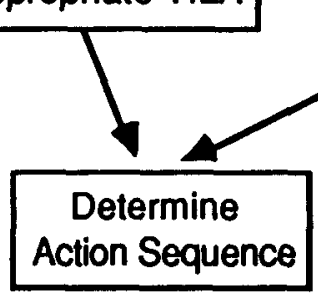

\section{Plan}

\section{Figures 4-5c and 4-5d}

Expanded versions of the six general performance model components 
procedures. This was referred to above as rule-based decision making. The third case, which is the purely knowledge-based approach to decision making, relies on the decision maker's ability to reason out a plan that will succeed.

It is assumed that decision makers will have available both the EOPs and the new SAMG (severe accident management guidance) or that the two will be integrated. Each embodies a plan to achieve a clearly defined goal. It is likely that the SAMG will be tied into the EOPs with transition points. For some owners' groups, the transition works only in one direction. That is, after the EOPs are abandoned for the SAMG, there will not be a return to EOPs. Also, it is likely that the SAMG will be less detailed than the EOPs. The SAMG may require decision makers to do some evaluation of "best candidate" high-level actions (HLAs).

One path for planning, then, is to find an appropriate connection in the EOPs and take the actions specified there. This approach, the rule-based approach, is the most straightforward. The model shows a doubleheaded arrow between the EOPs and the SAMG to account for transitions, especially in cases where the EOPs are no longer sufficient to address the transient and the SAMG becomes required.

The second path, then, is to use the SAMG to identify an appropriate HLA. The model allows for the possibility that some evaluation of HLA candidates may be required, and therefore, this approach may require both rule-based and knowledge-based performance. When the appropriate HLA is selected, the SAMG may provide a detailed procedure for taking action. However, there may be some requirement for decision makers to determine how to implement an HLA-that is, determine what specific actions in what order. To allow for this, a "determine action sequence" component has been included.

The third path for planning, the knowledge-based approach, is relevant when no EOP or SAMG exists to address the current plant state, a situation with a very low likelihood. In this case, the model includes a formulate-and-evaluate loop. A tentative plan is formulated and then evaluated mentally before it is implemented. If the evaluation (a mental simulation of implementing the plan) indicates the plan will not be successful or will cause undesirable consequences, the plan is abandoned and an alternative plan is formulated. This cycle continues until a plan is accepted. At that point any remaining details concerning implementation (e.g., order of actions, precautionary actions) are determined. Each of the three paths for planning leads to a set of control actions.

Control. Figure 4-5e shows that the control process has been expanded into two components. This expansion focuses on issues of resource management in taking non-routine control actions. That is, there may be a need for more complicated control actions that are not routinely considered. There may be a need, especially when planning is not done via a procedure, to establish and alter priorities of actions as plant state evolves, to allocate actions between operators when actions are not routine, and to coordinate control actions being taken in numerous locations. This resource management may also concern taking into account automated control systems and their effects. Thus, although control actions are typically a routine element of decision making, SAM situations may give it a non-routine character, especially if decision making is being done from the TSC.

Feedback. Feedback, shown in Figure 4-5f, was not explicitly represented in the original Rasmussen model. Others, adapting the model, have incorporated it explicitly over time (Woods, 1982). Making it explicit provides a greater sense of continuity in the performance model--that is, performance is seen as a series of decision-making cycles, each providing information that feeds into the next.

The expanded model of feedback identifies information at three levels. First, the operators receive feedback (monitor actions) from the controls that the control action was executed successfully (e.g., the switch is in a new position). Second, operators should see changes in parameter values or in indications of plant functions that are the result of the control action (e.g., flow is reduced, pressure decreases). At the highest level, operators are provided information about whether the control actions are supporting goal achievement. This is an opportunity to evaluate the plan that was adopted. Because a nuclear power plant transient can evolve slowly over time, the operator's understanding of the event may change over time. It is critical for the decision maker to monitor the plan periodically and 
Execute Action Sequence

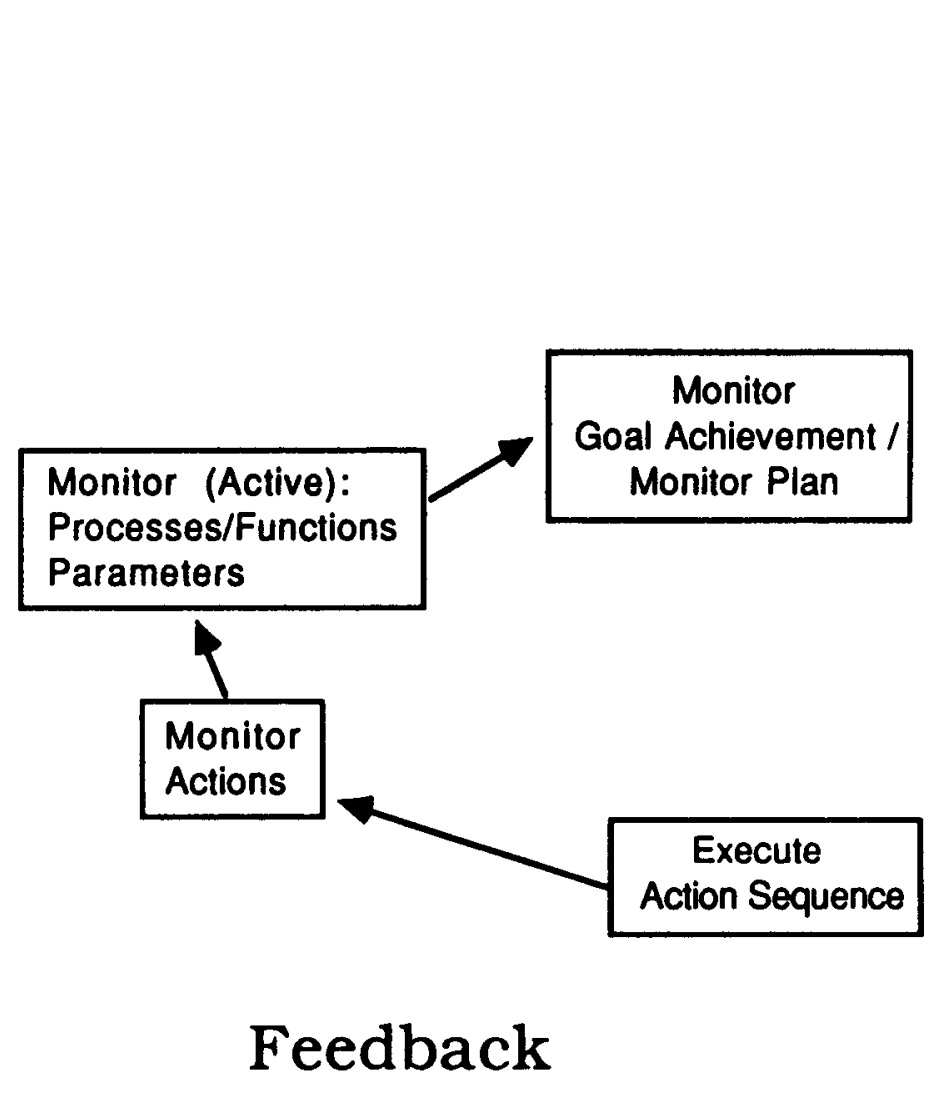

Feedback

\section{Control}


to evaluate progress in achieving the desired goals. The "monitor plan" component provides an opportunity to abandon the plan and rethink goals.

The Integrated Performance Model. The expanded versions of the six general processes have been integrated into a complete model of decision making in Figure 4-6. As stated previously, the primary function of this model is to be a representation of the critical decision-making processes required for SAM. The identification of these processes allows one to bring to bear what cognitive psychologists have discovered about each process so that the knowledge and skills that may be required for skilled performance and the sources of error that are likely in an SAM setting can be identified.

Figure 4-6 shows the general flow through the model and captures the general sequencing of processes. This does not represent all possible paths or present a detailed model of the sequencing of discrete cognitive processes. The discussion here provides a description of the important links between process components and briefly illustrates extreme cases of rule-based and knowledge-based decision making.

Figure 4-7 identifies three important transition points in the integrated performance model. These are points between performance model components where the selection of branches defines a rule-based or a knowledge-based path. The first transition point occurs from the Monitor / Detect section of the model, which terminates with the "integrate data" component. From "integrate data" there are two paths. In the case where the plant state data indicate a familiar event or set of symptoms, the decision maker can often select an existing emergency procedure (EOP) that will identify the appropriate control actions. It is possible that an arrow could branch to the SAMG as well (this additional arrow was left out only to reduce the clutter of too many arrows). When the plant indications do not lead to the selection of an EOP (or SAMG) in a straightforward way, the alternative path must be taken. This second path leads to "develop understanding of plant state," the knowledge-based path that allows the decision maker to develop a more complete picture of plant state.

The knowledge-based decision-making path requires two more transitions where the decision maker elects not to select an EOP or the SAMG. These transition points exist at "develop understanding of plant state" and "determine implications/establish goal hierarchy." In each case, by not selecting the rulebased alternatives, the decision maker places a major cognitive burden on the operations and TSC staff. Specifically, control actions must be determined from a lengthy reasoning process instead of from a procedure. As the integrated model shows, however, it is possible to make a transition from either component to the EOPs or the SAMG.

The simplest case of rule-based decision making is shown in Figure 4-8. In this case, it is assumed that the EOPs are sufficient for selecting control actions, and the TSC may not become involved in the transient. Again, the cycle often begins with the "detect /observe" component when an abnormal indication (e.g., alarm) is observed. The operators quickly select an EOP and execute the control actions specified there. Note that there may be some need for resource management in the control room ("prioritize /allocate/ coordinate actions"). Feedback occurs to inform operators that control actions have been executed successfully and parameters are responding appropriately. Feedback at the level of goal achievement is driven by the procedures and standard control devices, such as the critical safety function (CSF) tree. These mechanisms tell operators clearly when the symptoms have been eliminated or mitigated. The CSF tree makes this clear by assigning colors to each safety function. Thus, when a safety function goes from red (immediate action required) to green (safe), the operators receive feedback. Note that the "monitor goal achievement / monitor plan" component is somewhat outside the flow of the decision-making cycle. This scheme is intended to portray this component as a periodic activity not tied directly into the general sequence.

The four components of the performance model that are most representative of knowledge-based decision making are "develop understanding of plant state," "determine implications / establish goal hierarchy," "formulate response plan," and "evaluate response plan." These components will not be strongly involved when decision making can proceed in a rulebased fashion (although the first two components may always have some role even when decision making is rule-based). However, when decision making is knowledge-based, these four components play a major role. Figure 4-9 shows the performance model components required when decision making is 


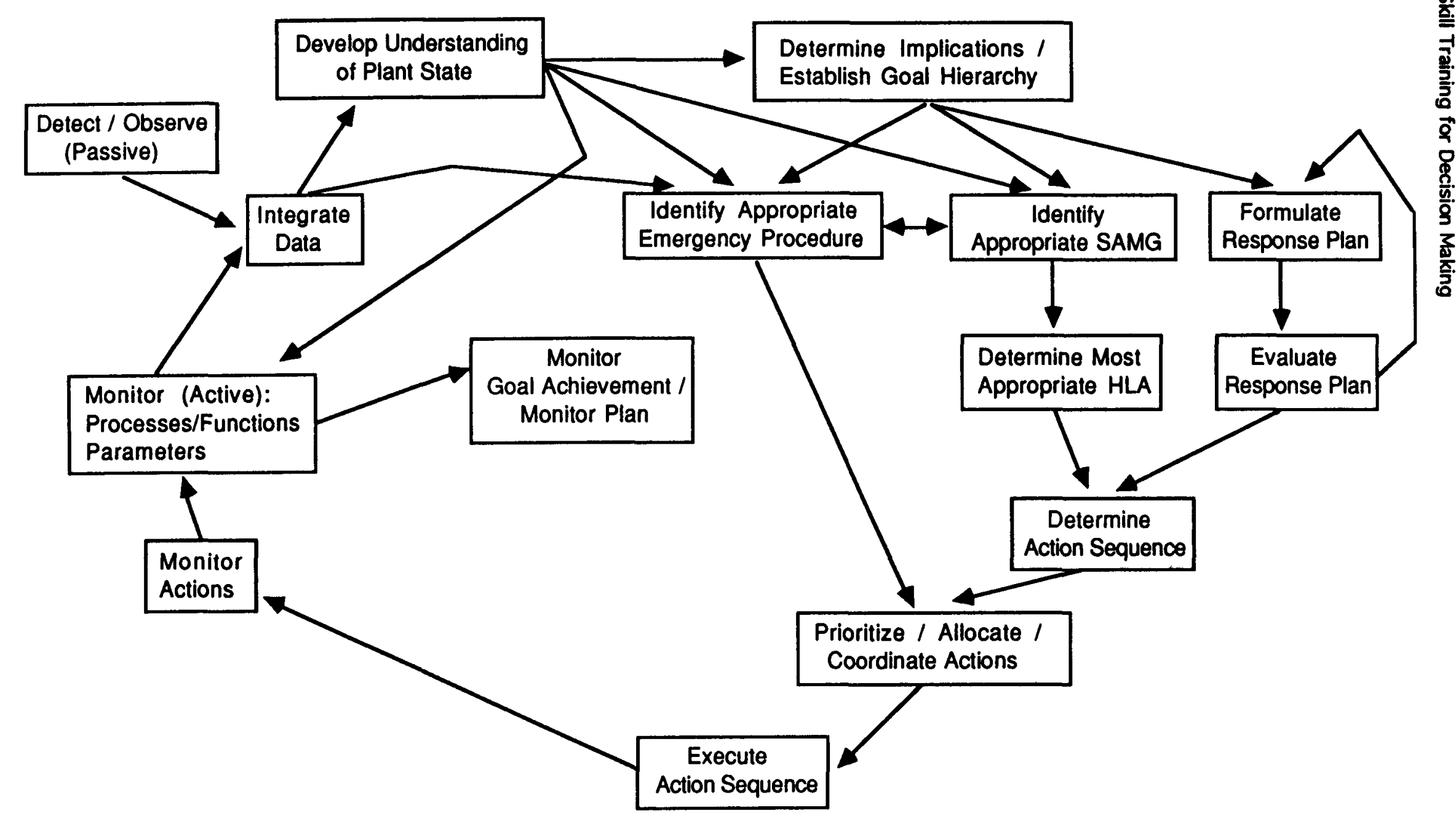

Figure 4-6. Integration of expanded performance model 


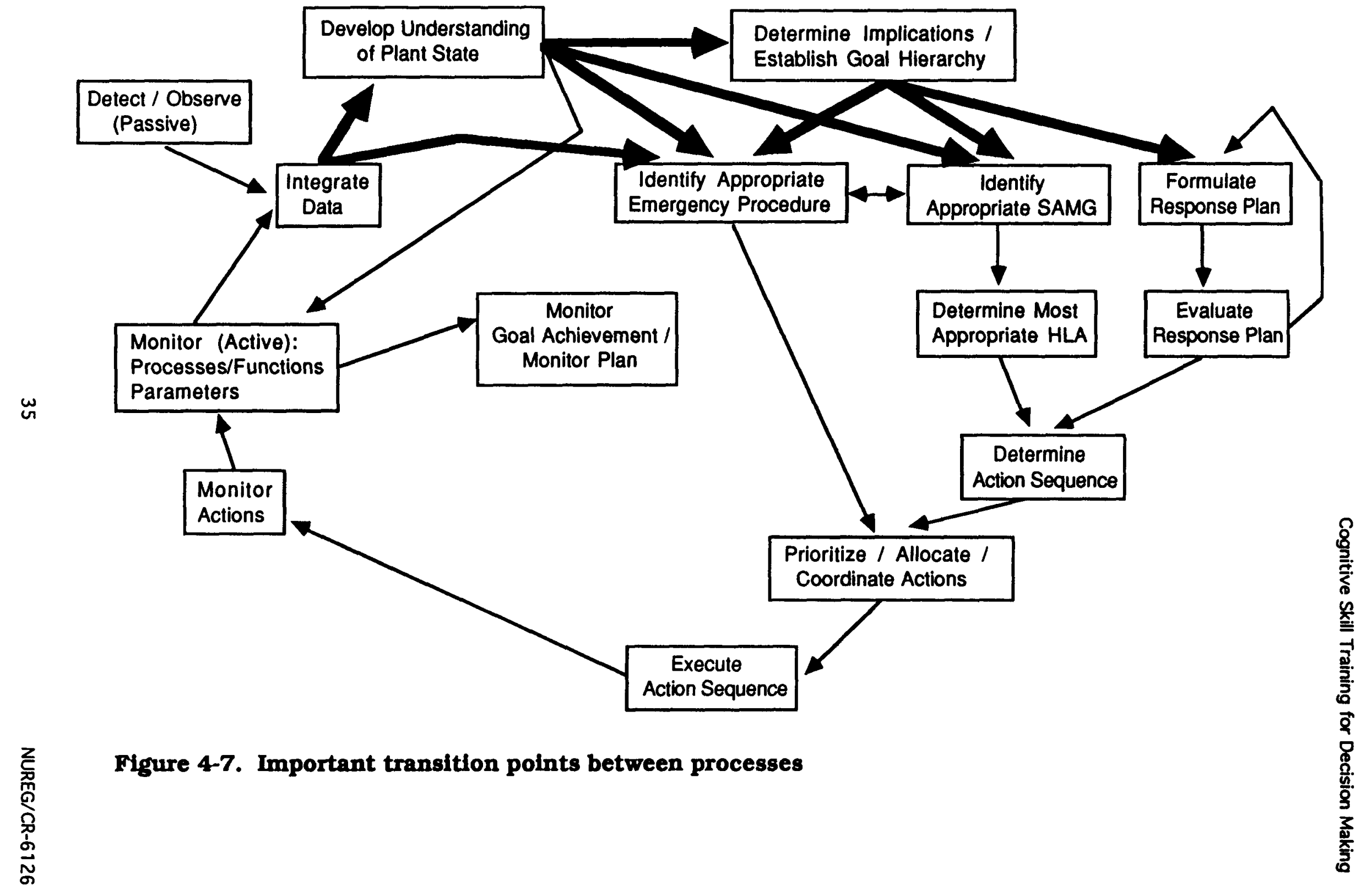




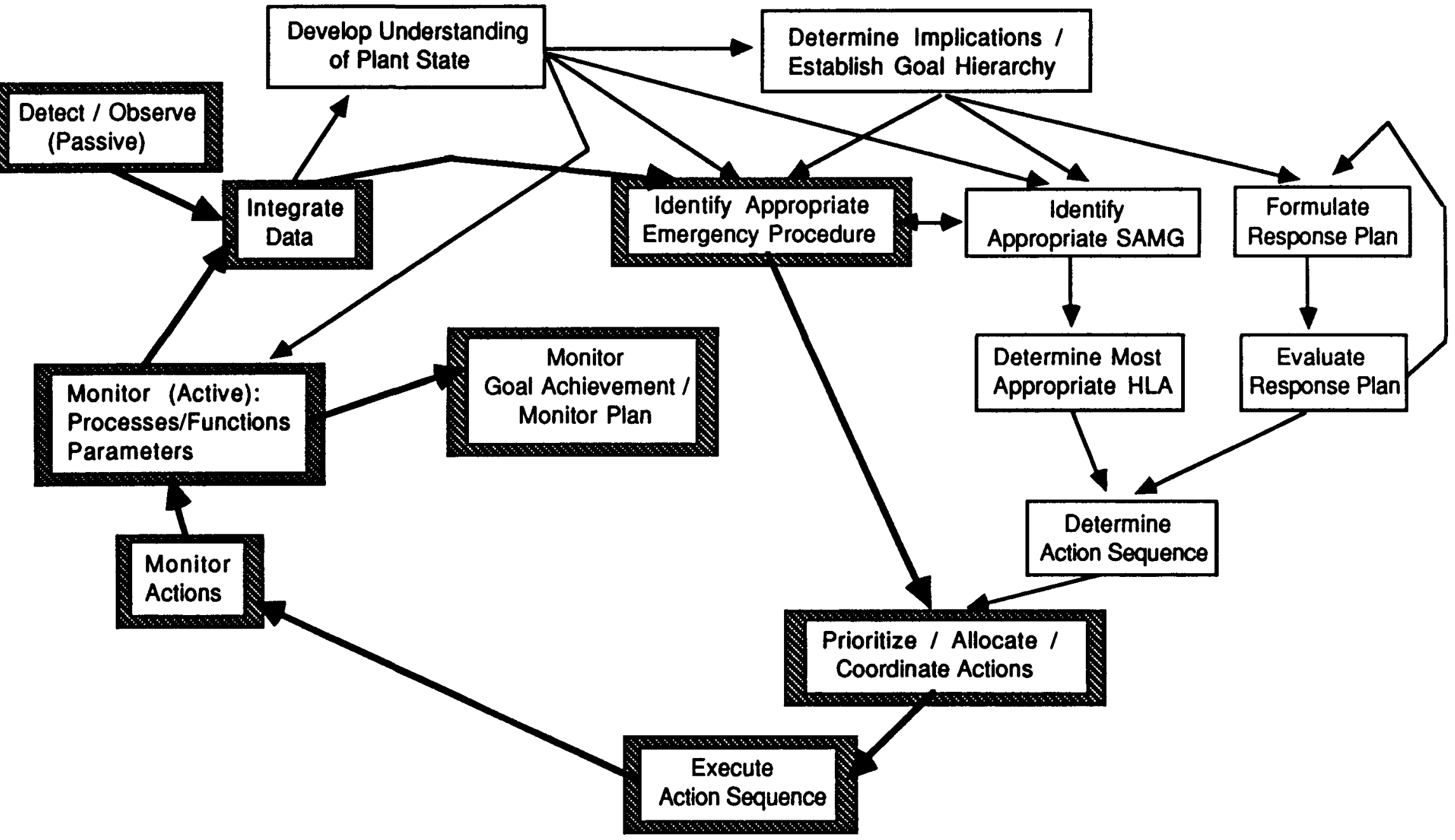

Figure 4-8. Performance model components used for simplest rule-based decision making 


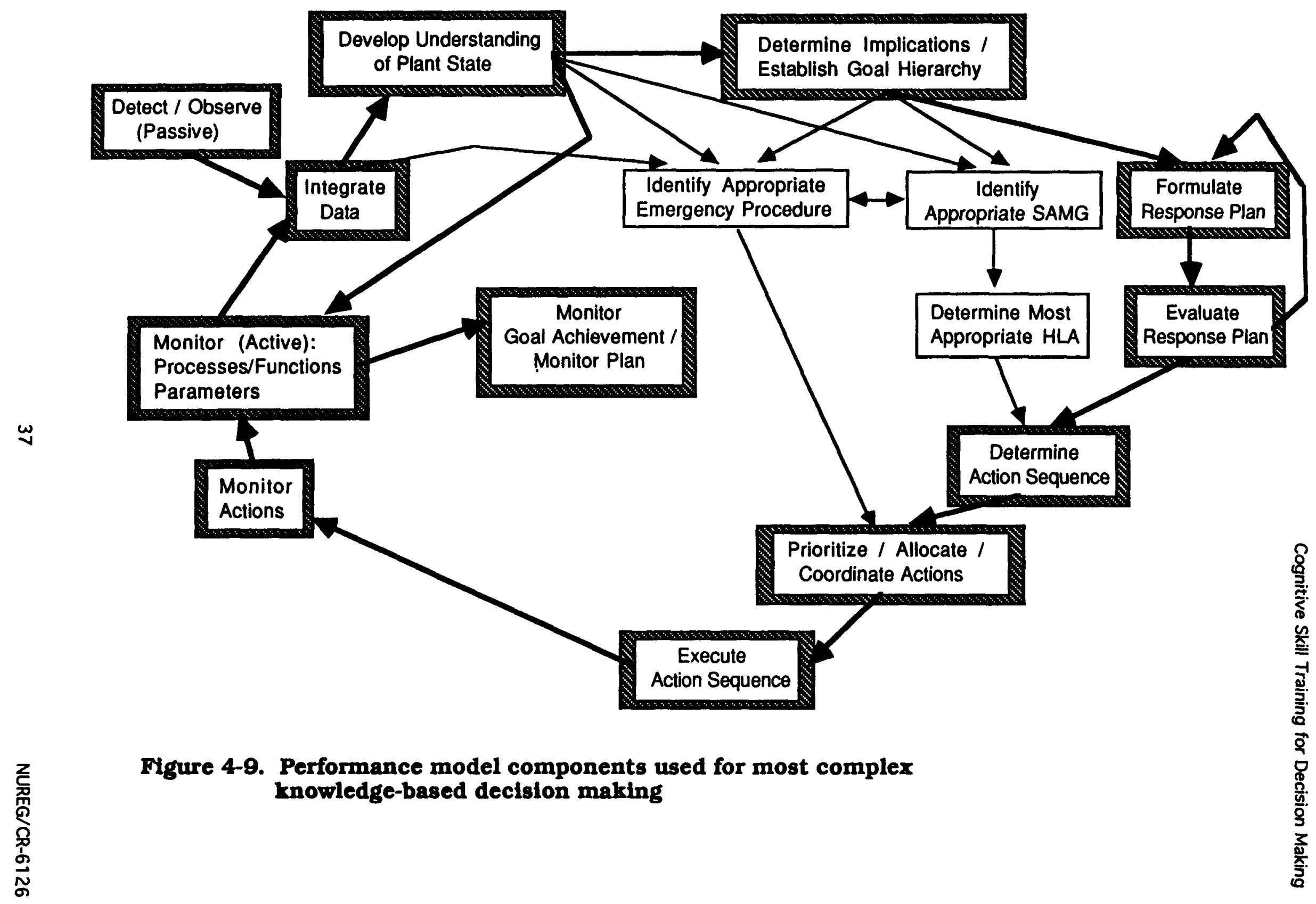


strongly knowledge-based. The case represented here is probably very unlikely to occur; it seems unlikely that the EOPs or SAMG will provide no guidance at all. Further, the "monitor goal achievement /monitor plan" component may become more complex when goals are determined by the decision maker. In other words, the CSF tree may not be a sufficient guide for monitoring goal achievement. Also, other events may occur that push the decision maker to abandon his plan.

As stated above, one goal of the performance model is to represent the major components of both rule-based and knowledge-based decision making. As a transient evolves from the first signs of an abnormal plant state to a severe accident situation, there are likely to be shifts in the components of the model that are relevant to actual performance. During an actual event, the EOPs and SAMG document will likely be sufficient to support performance--that is, performance will be strongly rule-based. The knowledge-based components are likely to take on a supplemental role, similar to that in the account of performance during difficult emergency events (see Section 3.1). However, the difference may be that the supplemental knowledge-based activity, instead of making performance more efficient, may become necessary to move through the SAMG correctly. Thus, the cognitive skills that often supplement skilled performance may become required for skilled performance. 


\section{Chapter 5. Cognitive Skills Required for Decision Making}

Chapter 4 provided a comprehensive performance model that establishes a starting point for analysis of the knowledge and skills required for NPP decision making tasks. In this chapter, that model is used to identify cognitive skills. The first analysis, illustrated in Figure 4-2, uses the model to identify cognitive skills for both individuals and the crew or team. The second form of analysis relies on an analysis of SAM scenarios. This analysis is described in Section 5.2.

\subsection{Identification of Cognitive Skills from Analysis of the Performance Model}

Using the performance model, one can begin a cognitive task analysis to identify the knowledge and skills required for severe accident management. The task analysis, as illustrated in Figure 4-2, should first address the knowledge and skills required for skilled performance in each of the six decision-making processes. That is, it is important to understand what knowledge and skills are needed to carry out decision making in an SAM setting. The primary input to this analysis are task analyses of the decision making required in similar settings. Completed analyses exist for nuclear power plant emergency operations as well as analyses of performance in fields such as electronics troubleshooting, medical diagnosis, and complex problem-solving tasks. These analyses provide a description of expertise that is contrasted to a description of novice performance. This contrast reveals the changes that occur in knowledge and skills over the course of acquiring a complex skill, and, therefore, provide prescriptions for training.

Typically, an analysis of performance is conducted for each individual. However, it is also important to look at team skills that are required for successful performance. Although cognitive skills are commonly trained at the individual level, the nuclear power plant setting requires strong coordination of team members. Therefore, because team training must be considered as well as the training of individuals in the SAM setting, our analysis attempts to identify critical team skills.
In addition to analyses of skilled performance, the :task analysis should address the likely impediments to skilled performance (i.e., common errors and biases). Humans have inherent cognitive limitations (Miller, 1956; Simon, 1957) that are likely to have an impact on performance in the demanding situation created by SAM. Indeed, a major component of acquiring expertise is reducing or eliminating the cognitive limitations, and a goal of training should be to provide a means for personnel to avoid situations that exploit cognitive limitations. A good source of information on likely human error in SAM is the literature on human errors in complex decision tasks. The task analysis incorporates documented analyses of human error in nuclear power plant settings as well as other settings (e.g., aviation, medical diagnosis).

This section is organized in six subsections that correspond to the six decision-making processes. Each of these sections is, in turn, divided into subsections that address the following:

- Situational Factors: important factors concerning the circumstances under which decision making occurs

- Cognitive Limitations and Biases: cognitive limitations and biases that are relevant to each decision-making process.

- Cognitive Skills - Individuals: the grouping and enumeration of specific knowledge and skills required for each decision-making process. Specific knowledge or skill as either a "Knowledge of ..." or "Ability to ..." is described. In many cases, there are close ties between a specific knowledge and the ability to apply it. The need for a skill or ability with descriptions of related human failures in documented accidents is also provided.

- Cognitive Skills - Team: the grouping and enumeration of specific knowledge and skills connected to team performance. These skills are often high-level skills that address the management of the task-that is, metacognitive skills. 


\subsubsection{Monitor/Detect}

As shown in Figure 4-5a, this decision-making process involves three components of the performance model:

- Detect /observe

- Monitor parameters /monitor processes and functions

- Integrate data

Situational Factors. The primary concern here is with acquiring accurate and complete indications of the plant state. However, plant state conditions can become seriously degraded as a transient evolves into a severe accident, especially when there are significant changes to the core. This evolution can have a significant effect on plant state data. The first effects are felt in the control room. First, data can be lost; instruments, sensors, and indicators can fail (e.g., indicator goes off-scale). Also, indications that may be very useful for SAM are not instrumented (e.g., little information is available on core status).

Second, data can become misleading or unreliable. As conditions move out of instrument boundary areas, there is the threat of false readings or false alarms (e.g., as temperature increases through a certain area, instrument failure becomes more likely). The existence of misleading data or uncertain data (in excess of what is common in control rooms) reduces the confidence that is assigned to plant state indications. Further, it is possible that if instruments are forced into unusual value ranges, they may not provide sufficient sensitivity or context to support diagnosis. Others have documented cases in which instruments (due to insufficient discriminability) did not support diagnosis as effectively in unusual value ranges (Hoecker, Pople, \& Benhardt, 1991).

After the TSC is manned and decision-making authority is passed to TSC personnel, the acquisition of accurate and complete data by decision makers may become even more difficult. A primary consideration is the effort required for TSC personnel to understand what has occurred prior to their involvement. The accident may be 1 to 2 hours old before they reach the TSC, and the supporting documentation that is available (e.g., alarm histories) may contain too much information to separate the relevant from the irrelevant easily. Briefings from control room personnel will be one of the main sources of information, and a means to bring together all relevant information in one place to facilitate the construction of a coherent picture of the event's progression will be needed.

After TSC personnel have been brought up-to-date, other problems can arise. First, the access to data is limited. Instead of having all indicators constantly available, TSC access to plant state indications may be restricted to a small number of parameters at a time. This limited access introduces the issue of data integration, both in time and space. TSC staff may have difficulty integrating individual indications in order to develop a complete picture of the plant. Second, the display devices in the TSC may not provide as much information on data reliability or data context. Changes in display format can also remove information (e.g., parameter rate of change) that is easily determined in the control room. Finally, the TSC staff, when compared to control room decision makers, may rely more heavily on data reported through voice communications from the control room or other areas of the plant. This source of data is also susceptible to misinterpretation, false alarms, and lost context. Phoned-in data are also more transitory; if they are not recorded by someone in the TSC immediately, they may be lost or forgotten.

Cognitive Limitations and Biases. The primary issue regarding cognitive limitations in this process concerns limitations in attention and working memory. As stated above, working memory refers to the crew member's current consciousness--that is, the set of items being considered. Studies of this type of memory (Miller, 1956) indicate that humans can typically retain about seven separate items in working memory. Although there are limits on the number of items, the size or complexity of each item can be increased to increase capacity. Due to the limits, new information can "bump out" current items before they are incorporated into long-term memory. These items may then be lost. Loss of information is very likely in a nuclear power plant where indications can change rapidly. Although some indicators provide a history or trend plot, many parameter values can be lost if they are not attended to at the appropriate moment.

The early stages of abnormal events, where detection is the primary concern, are often characterized by an overwhelming number of alarms and parameter changes. Because of cognitive limitations, control room personnel are severely limited in the number of 
indications or alarms they can attend to at any time or can keep in working memory. This limitation requires some filtering of information. That is, because only a handful of indications can be considered at any time, there is a need to select that handful and ignore other indications. The crew members must attend selectively to the indications they believe are most relevant and relegate other indications to a lower status. If an operator can attend to the handful of most important indications each time he seeks information, the impact of cognitive limitations can be reduced. Support for this filtering comes primarily from the next process, Interpret Current State.

Cognitive Skills - Individuals. At the highest level of cognitive skill analysis for this process, three skills have been identified: the ability to determine accurate indications of plant state, the ability to integrate plant state indications, and the ability to identify meaningful events. These are the critical precursors to the development of an accurate and complete mental representation of plant state. The following describes each of these high-level cognitive skills and lists the lower-level knowledge and skills that are likely to be critical.

1. Ability to determine accurate indications of plant state. Skilled performance requires determining which data are valid indicators of plant state and identifying the most reliable indications. Also, skills need to be developed to overcome the many impediments to data gathering that can occur during SAM.

Lower-level knowledge and skills are:

- Knowledge of environmental limitations on instrumentation.

- Ability to identify the conditions under which back-up indications are needed.

- Ability to recognize failed or unreliable instruments and discriminate them from an instrument showing an appropriate response to abnormal conditions.

- Knowledge of less direct indications that back up primary indications.

- Ability to obtain the most reliable indication available.

2. Ability to integrate plant state indications. Skilled personnel (especially TSC personnel who have limited access to data) need to be able to develop fairly complete "snapshots" of the plant state by integrating the relevant data. This is critical initially when they first man the TSC and attempt to understand what has transpired prior to their arrival. It remains an important skill as the event progresses and they try to monitor its progression.

Lower-level knowledge and skills are:

- Ability to integrate all relevant data in space and/or time.

3. Ability to identify meaningful events. Detection and monitoring of plant state data do not serve simply to make staff aware of individual data values.

They need to be supported in isolating patterns of certain data that define meaningful configurations or combinations of plant state data ("events"). The control room or TSC interface (i.e., set of displays) does not always organize plant state data in a manner most convenient for identifying meaningful events. Skilled personnel should be able to scan the interface to detect these events when they are present.

Lower-level knowledge and skills are:

- Knowledge of data display and alarm organization.

- Ability to scan control room (or TSC) interface in a systematic way and recognize meaningful data patterns rapidly.

Cognitive Skills - Team. The major consideration for team performance in this decision-making process is communicating plant state indications to the decision maker. This may be especially critical when the decision-making role has moved into the TSC. Compared to when the decision maker is in the control room, the TSC gives communicators more limited access to the decision maker, or at least less direct access.

\section{Ability to communicate important plant state} indications to the decision maker. It is critical for the team to report plant state indications to the decision maker in a way that allows rapid incorporation of the information into a developing picture of current conditions. Communication failures can have several sources. While some involve the mechanics of communication (voice link fails, operator reports to the wrong person, etc.), the more important failures involve cognitive aspects of communication. These are cases in 
which some communication is made but the information communicated is not dealt with appropriately. Much of the meaning of spoken communication relies on a shared context that is not always preserved or communicated in voice links. Typical failures involve improper referents and hidden assumptions.

Lower-level knowledge and skills are:

- Ability to communicate plant state indications in a timely and accurate manner.

\subsubsection{Interpret Current State}

The primary consideration of the Monitor / Detect process, described above, is the acquisition of accurate and complete indications of plant state. The focus in the Interpret Current State process is the development of a mental representation of plant state-that is, an interpretation of the set of indications available. Figure 4-5b shows the three components of the performance model related to this process:

- Monitor parameters /monitor processes and functions.

- Integrate data.

- Develop understanding of plant state.

This process is associated with knowledge-based decision making (compare Figures 4-8 and 4-9). Thus, when rule-based decision making is sufficient to drive performance, this process is not required (although it may be beneficial).

Situational Factors. Current nuclear power plant procedures are built on the assumption that rulebased performance can be driven by single indications (or simple conjunctions of single indications). Each procedural branch is tied to an alarm or parameter, and there is, on the surface, no need to describe plant state at a higher level. Therefore, if a need arises for a higher-level description of plant state, it is largely up to the control room and TSC crews to give interpretation to the large set of plant state indications presented via the various plant interfaces (refer back to the description of operator decision making in Section 3.1). This interpretation can exist at several levels and may include descriptions of faults or root causes (e.g., ruptured pipe), the status of physical components (e.g., steam generator, pressurizer), the status of functional components (e.g., feedwater system), or the status of pre-defined safety functions (e.g., core cooling).

One component of interpretation is the acquisition of plant state data that can help narrow or refine the interpretation. Interpretation cannot proceed unless one can identify and integrate relevant and reliable data. Therefore, the situational factors that apply to the Monitor / Detect process need to be considered as factors in this process as well. Current plant interfaces do support personnel in identifying a small set of safety parameters. Specifically, the safety parameter display system (SPDS) brings together in one location a set of safety parameters that are otherwise distributed around the control room. In a $B W R$, these parameters include reactor vessel pressure, level, and power. Thus, although no interpretation or higher-level description occurs, a small set of parameters are integrated spatially to aid operators and TSC personnel in identifying relevant plant state data.

The more important component of interpretation is the integration of data to describe plant state as something more than simply the collection of many individual indications. The PWR control room interface supports this activity with the critical safety function display. In all PWRs, control room and TSC - staff are able to use a special display to determine the status of six critical safety functions. These functions are the following:

- Subcriticality

- Core cooling

- Secondary heat sink

- RCS integrity

- Containment integrity

- RCS inventory

The status of each function is indicated by one of four colors (green, yellow, orange, and red) on the computerized display. Green indicates a satisfied safety function, red indicates the most extreme challenge, and orange and yellow represent less severe challenges. The status of each function is determined by integrating a number of individual parameters, which can also be done manually if the computerized display is unavailable. This display is the only support provided by the plant interface for interpretation. Any interpretation beyond this must be carried out in the heads of the control room and TSC crews. 
Cognitive Limitations and Biases. There are a number of significant cognitive limitations and biases that can hinder the development of an accurate and complete mental representation. They are described here under the following general headings:

- Garden path interpretations.

- Representational limits.

- Confirmation bias.

1. Garden path interpretations. A schema (which may also be referred to as a script) was previously described as a generic representation of a familiar or well learned event, and a restaurant example was offered. A restaurant schema describes the elements of the event and their interconnections. It establishes expectations about the outcome of an event. Thus, if one goes to a new restaurant, the schema provides expectations about what will occur. It provides a way of interpreting each action as part of a familiar event.

A schema is a powerful tool for interpreting a new event, and humans have been shown to exploit this tool whenever possible to identify familiar elements or patterns quickly, invoke the schema tied to those patterns, and interpret actions more efficiently. The schema guides the interpretation, and a schema invoked by a nuclear power plant transient can aid the interpretation process in a similar way. It allows one to make inferences more easily, to identify relevant data, to anticipate later events, etc. Problems can arise, however, when an inappropriate schema is invoked. That is, one may respond to a familiar pattern that is not a representative symptom of the actual event (e.g., the pattern is tied to just one of multiple failures). This over-reliance on familiar patterns is referred to as "leading one down the garden path." A garden path interpretation is one that is based on a highly familiar pattern or cue that turns out to be misleading.

The danger of the garden path interpretation is that decision makers believe they understand the plant state when they do not. There are a number of possible results: a) they become fixated, monitoring only data tied to their interpretation and ignoring other plant state data that may be important; b) data revealed to them that disconfirm their interpretation may be discounted because they do not fit into expectations; and c) they may stop monitoring plant state data because they believe their interpretation is complete.

2. Representational limits. One reason a schema is powerful is because it organizes a great deal of information in a single representation in long-term memory; and, as was stated previously, knowledgedriven organization of memory can help overcome the normal limits on processing. When no schema is invoked, a mental representation must be built up over time to incorporate the inferences being made. Humans have severe limits on the amount of information that can be incorporated into a single representation (Simon, 1957). When limits are reached, the representation can fail to work-that is, fail to support further inferences, fail to generate expectations about plant state, fail to identify relevant data, and fail to account for all the data (e.g., one may not notice that two inferences are contradictory). The resulting simplified or incomplete representation is often not sufficient for knowledge-based decision making, and performance is poor.

A second concern, which applies both to cases in which a schema exists and cases in which representations are built from inferences, is the presence of incorrect inferences and misconceptions. Representations are powerful to the extent they are correct. However, when incorrect information exists (about what an indication means, about connections between components, etc.), the representation is likely to cause one to make incorrect inferences or to ignore disconfirming data.

3. Confirmation bias. Skill in developing a representation includes skills for monitoring plant state data in an active way to confirm, disconfirm, or differentiate an interpretation based on earlier indications. Indeed, because nuclear power plants are composed of dynamic, tightly coupled components, it is probably wise to monitor an interpretation continuously until the transient is mitigated. Because of the risk of garden path interpretations, it is especially important to seek information that may disconfirm the current interpretation. However, a number of studies have shown that humans in similar situations are biased to seek confirmatory information only (for a review, see Evans, 1989). That is, a decision maker is likely to use his interpretation to generate an expectation about plant state data, and then actively seek out the data to confirm that expectation. It is unlikely, though, that he would seek 
data that could disconfirm his interpretation or confirm other interpretations. In some cases, both confirming and disconfirming data are needed. The risk in this case is that the decision maker will develop an interpretation that is incomplete or incorrect.

Cognitive Skills - Individuals. The most desirable product of this process is a complete and accurate interpretation--a mental representation that can support decision making by identifying the primary cause(s) of a transient and the pattern of influences within that system and related systems. This representation can support the setting of goal priorities and the selection of control actions. It is unlikely that a representation of this quality can be developed from the initial set of indications. Typically, an initial interpretation is established and then tested and enhanced through further monitoring of plant state data (the process loop in Figure 4-5b). Or, it may be that a small set of alternative interpretations is considered until further plant data support one and exclude the others. Therefore, an effective representation also provides a means for generating expectations about parameter values--that is, it determines the plant state data that can confirm an interpretation, disconfirm an interpretation, or differentiate between competing alternatives.

Such a comprehensive interpretation of plant state is not required to support decision making. Even when the crew can use single indications to select a procedure, a less detailed representation is often useful for anticipating a transient's progression, judging the value of discretionary actions, or reducing uncertainty in general. For example, in PWRs, the identification of a safety function violation is sufficient to select a procedure and take control actions. However, the more complete and accurate a mental representation is, the better it supports any knowledge-based decision making that is required.

At the highest level of cognitive skill analysis for this process, three skills have been identified: the ability to make inferences about the current plant state, the ability to determine expected influences and relevant data, and the ability to recognize links to existing procedures and SAMG. These are critical skills for supporting decision making and the use of any procedure or guidance document. The following describes each of these high-level cognitive skills and lists the lower-level knowledge and skills that are likely to be critical.

1. Ability to make inferences about current plant state. To develop a mental representation, crew members must begin by integrating plant state data to make inferences about the causes of the transient or the current status of equipment and systems. Inferences may be made in a piecemeal fashion so that a complete representation or a representation of major components is developed over time. The identification of a safety function violation may be an inference made early on before a more complete representation is developed. Or, crew members may recognize a set of indications as a pattern associated with a well understood event. For both PWRs and BWRs there is a small set of designbasis events that are thoroughly studied by crews. Each of these events is associated with a set of indications (symptoms). When this set of symptoms is present, crew members can instantly create a fairly rich representation that captures the generic event. For example, PWR crew members should have a generic steam generator tube rupture (SGTR) representation that will be invoked when a certain set of symptoms is present. This representation, called a schema, provides expectations about related symptoms and has placeholders for more specific information.

Lower-level knowledge and skills are:

- Knowledge of plant components, plant systems, and their relationships.

- Knowledge of current plant-specific conditions (faulty equipment, tag-outs).

- Knowledge of important physical phenomena during severe accident progression.

- Knowledge of criteria for violating critical safety functions.

- Ability to make inferences about:

- set of initiating conditions for transient

- existing faults, breaks, failures, losses

- safety function status

- core, vessel, and containment status

- availability of safety systems

- Knowledge of design-basis events and their symptoms.

- Knowledge of high-probability severe accident events and their symptoms.

- Ability to develop complete and accurate mental representation of plant status. 
2. Ability to determine expected influences and relevant data. As stated above, after an interpretation has been developed, skilled performance includes the ability to verify and/or refine the initial representation. This process is an iterative exercise in generating expectations about plant state and verifying them through an inspection of relevant plant data. Thus, the initial interpretation leads to the filtering of data that is required to keep from being overwhelmed by the flow of plant data. For example, if an inference has been made that a fault exists in component $x$, crew members must determine how that fault influences other components and systems. This generates expectations about the values of plant state data, which in turn focuses attention on some data to the exclusion of other data. When the expectation is verified, the interpretation is supported.

Lower-level knowledge and skills are:

- Knowledge of pattern of influences (in systems, in components) created by current plant conditions (e.g., specific fault).

- Ability to mentally simulate normal and abnormal influences to develop expectations about plant behavior.

- Ability to identify specific markers/cues that can confirm primary interpretation.

- Ability to identify plant state data or data patterns that can differentiate between competing interpretations or disconfirm primary interpretation.

3. Ability to recognize links to existing procedures and $S A M G$. There is the need for a link from the Interpret Current State process back to rule-based decision making. It is possible that knowledgebased activities will reveal a description of plant state that defines a branch into existing procedures or the SAMG. The performance model allows for transition to these components.

Lower-level knowledge and skills are:

- Knowledge of emergency procedures and SAMGs and their functions.

- Knowledge of criteria for violating critical safety functions.

- Knowledge of criteria for selecting procedure (the conditions for which procedures apply).

- Knowledge of criteria for timely actions (e.g., feed and bleed, RCS depress).
Cognitive Skills - Team. It is likely that each person in the control room and TSC will, over the course of a response to some transient, begin to make inferences about the state of the plant--that is, each person will independently develop some representation. Further, each crew member will have access to a set of data somewhat different from the set others can easily access. These differences are likely to lead to inferences and representations that do not overlap completely. The primary decision maker has limited resources for drawing inferences and seeking out data, and he needs support from other crew members. The best chance for the development of an accurate and complete representation occurs when the resources of the entire team are used.

Thus, if the crew is aware of the decision maker's interpretation of events, they can seek confirming and disconfirming evidence. This activity can be carried out in spare moments as an incidental task. It is not necessary to devote others to this task explicitly. The goal is to make crew members aware of the decision maker's interpretation in enough detail so they can identify and communicate information that disconfirms (or differentiates).

1. Ability to provide team input to interpretation process. A method or a technology is needed that supports the crew in sharing information about plant state and the data that address the current interpretation.

Lower-level knowledge and skills are:

- Ability (decision maker) to communicate primary inferences or other interpretations to crew members.

- Ability (crew members) to communicate plant state data that confirm or disconfirm current interpretation.

\subsubsection{Determine Implications}

After a mental representation of plant state has been developed, a course of action must be established. The focus in this process is to use the mental representation to anticipate the event's course, to identify goals for action (i.e., determine what needs to be accomplished), and set priorities for those goals. At this point, it may be possible to return to rulebased decision making by identifying an appropriate procedure or SAMG. These documents set priorities for goals and spell out the actions required to achieve 
each goal. If it is not possible to select an existing procedure, the decision maker is forced into knowledge-based decision making. As stated in earlier sections, even when a procedure is available, cognitive skills may be applied to benefit control room and TSC staff in forming their own impressions about priorities in the event that the SAMG requires some evaluation of options. This process is split into two components (as shown in Figure 4-5c):

- Determine implications

- Establish goal hierarchy

The SAMG, while still having many of the same elements as the EOPs, is likely to differ in two ways. First, it may offer options on which goal should be given top priority. Second, it may offer options on which method is most appropriate for achieving a goal. Thus, when compared to current practices, the SAMG may introduce:

1. New goal priorities: The decision maker has to deal with more than one of the goals defined by the procedures. There may be a need to make trade-offs (i.e., violate one goal in order to satisfy a second) or to find a means to satisfy multiple goals with one action.

2. New method to achieve goal: The decision maker has identified a goal addressed by the procedures, but the procedures' method to achieve the goal cannot be implemented, and a new method is required. Or, perhaps multiple methods are available, but a decision needs to be made about which is most appropriate.

These elements of decision making may be difficult because in current EOPs goals are often an implicit, not explicit, component of procedures. Without a reorientation or training program, operational and TSC staff may have difficulty in thinking about goals and goal priorities. Goal priorities may be thought of as fixed, not as something that can be adapted to circumstances.

Situational Factors. In terms of purely knowledgebased decision making, the control room or the TSC will not provide decision aids for using a mental representation to anticipate the event's course, identifying goals for action (i.e., determine what needs to be accomplished), or setting priorities for those goals. In some sense, the decision maker is on his or her own, except for help he or she may receive

NUREG/CR-6126 from others (or from the SAMG). Indeed, identifying goals and setting goal priorities may be the first point in accident management where others outside of the control room and TSC actively try to become involved in decision making.

Cognitive Limitations and Biases. The primary considerations for this process are representational limits, a concept that was introduced under the Interpret Current State process. Because there is so much reliance on the representation to simulate the progression of a complex process, the representation must be very detailed. It is unlikely that an effective level of detail can be supported when severe accident phenomena are not well understood. Failure of the representation to support this mental simulation can lead to a simplified or incomplete representation. This, in turn, can lead to incorrect conclusions or gaps in the side effects that are anticipated. Incorrect information or misconceptions in the representation can lead to faulty conclusions as well. Obviously, an incorrect representation can lead to major failures as well. Thus, reliance on a garden path representation becomes a concern.

Cognitive Skills - Individuals. It seems unlikely that this process will be required to support purely knowledge-based decision-making. The assumption is that the crew will be able to select an existing procedure or SAMG. However, this is one area in which cognitive skills that supplement the SAMG, or make use of the SAMG possible, are very important to skilled performance. Thus, situations need to be considered in which the decision maker will make use of a mental representation as a simulation to anticipate the event's progression and determine goals, or to weigh options presented in the SAMG document. It is possible, as the SAM scenarios in Appendices A and B illustrate, that plant indications identify more than one goal as important. In this case, the decision maker must determine how to set priorities for goals. Difficulties arise when achieving one goal risks violating another, when the standard goal priorities must be adjusted, and when multiple goals must be satisfied simultaneously.

At the highest level of cognitive skill analysis for this process, three skills have been identified: the ability to use a mental representation to simulate an event's progression and identify goals, the ability to determine goal priorities, and the ability to recognize links to existing procedures and SAMG. These are 
required to support purely knowledge-based performance, and are probably very important to support the use of the SAMG document. The following describes each of these high-level cognitive skills and lists the lower-level knowledge and skills that are likely to be critical.

\section{Ability to use a mental representation to simulate an} event's progression and identify goals. The representation developed by the decision maker needs to support him in anticipating the progression of the event. Generally, the decision maker needs to determine what is likely to occur and when it is likely to occur. More specific questions may be "What systems or components will be affected or lost?" "How will critical safety parameters be influenced?" "How much time is available to mitigate this situation before barriers are breached?" This task may be difficult since the progression of certain severe accident phenomena is not well understood.

Lower-level knowledge and skills are:

- Knowledge of critical physical phenomena during severe accident progression.

- Knowledge of plant system interconnections and relations.

- Ability to anticipate the phenomenological consequences of action or inaction.

- Ability to anticipate the side effects of actions on goals.

- Ability to identify important goals for control actions.

2. Ability to determine goal priorities. In the case that multiple goals need to be considered, the decision maker needs to be facile in setting priorities, and in evaluating the validity of goals suggested by others and eliminating those that are inappropriate.

Lower-level knowledge and skills are:

- Ability to determine necessary subgoals.

- Ability to weigh multiple factors to set goal priorities (imminence, severity of potential consequences, knowledge of risk, etc.).

- Ability to determine whether a goal is valid.

3. Ability to recognize links to existing procedures and $S A M G$. As in the Interpret Current State process, there is the need for a link back to rule-based decision making. It is possible that knowledge- based activities will reveal a goal that is adequately addressed by existing procedures or the SAMG. The performance model allows for transition to these components.

Lower-level knowledge and skills are:

- Knowledge of emergency procedures and SAMGs and their functions.

- Knowledge of criteria for violating critical safety functions.

- Knowledge of criteria for selecting procedure (the conditions for which procedures apply).

- Knowledge of criteria for timely actions (e.g., feed and bleed, RCS depress).

Cognitive Skills - Team. As is the case in the Interpret Current State process, the primary decision maker has limited resources for the difficult cognitive tasks required here, and needs support from other crew members. The best chance for the effective identification of goals occurs when the resources of the entire team are used. It will be difficult to include others unless some technique is developed to communicate the intermediate results of the decision maker's mental simulation.

\section{Ability to provide team input to goal identification} /priority setting process. There needs to be a method or a technology that supports the crew in sharing information concerning how the event will progress and what goal priorities should be set.

Lower-level knowledge and skills are:

- Ability (decision maker) to communicate intermediate results of his mental simulation to crew members.

\subsubsection{Plan}

The primary consideration in this process is selecting or developing a plan for achieving the goal(s) established in the previous process. A plan can be selected in the cases where there is an appropriate procedure or SAMG (rule-based decision making). A plan must be developed in the unlikely case where no appropriate procedure exists (knowledge-based decision making). Figure 4-5d shows how these two approaches are represented in the performance model. The process components for the rule-based and knowledge-based decision making are the following: 
Rule-Based (Select Plan):

- Identify appropriate emergency procedure

- Identify appropriate SAMG

- Determine most appropriate HLA

- Determine action sequence

Knowledge-Based (Develop Plan):

- Formulate response plan

- Evaluate response plan

- Determine action sequence

Situational Factors. Rule-based decision making (i.e., use of the SAMG) should be fairly well supported during SAM. Decision makers are likely to be given several options for achieving their goal and be asked to select one. Their selection is likely to be based on a time window, equipment availability, and an assessment of the likely consequences of each action sequence. However, there is likely to be a great benefit gained from cognitive skills that supplement the SAMG use, especially for components such as "determine most appropriate HLA" and "determine action sequence."

In the case of knowledge-based decision making, decision makers are more on their own to develop a general plan and the sequence of control actions that will achieve that plan. The primary support for this activity are the many plant documents available to decision makers--for example, piping and instrumentation diagrams, piping flow diagrams, control logic diagrams, electrical schematics, and power distribution diagrams. These documents are available to the control room and TSC staff.

Several situational factors can complicate knowledgebased planning. First, as with the Determine Implications process, other people will become involved (utility people, NRC, etc.) and may have useful information or advice. Communication needs to be managed, however, so there is not a significant additional workload required for addressing each concern.

A second important factor is the combination of time pressure and uncertainty. Time pressure comes from the need to mitigate the event as quickly as possible. It is likely that the plant state will continue to worsen after a severe accident has been initiated. In some cases, the crew can estimate how much time is available before a significant change occurs (e.g., core becomes uncovered). In other cases, the crew will not be able to make accurate estimates about time. In either case, there is likely to be pressure to take action quickly. The second part of this situation is uncertainty about the current state of the plant. Although it is assumed that, at this point in decision making, the decision maker will have formed a representation of plant state, in many cases that representation will include uncertainty. As was described earlier, the decision maker can have difficulty in obtaining reliable and meaningful indications of plant state. The decision maker's perception may be that his understanding of plant state will improve as time passes. Thus, data uncertainty says "wait," and time pressure says "act." The result is a great deal of stress in a situation that requires a heavy mental workload.

Pressure to wait can also come from the uncertainty of risk associated with some actions. A plan, or a procedure, may require the crew to take an action that has adverse effects on plant state (e.g., bleed and feed in a PWR to resolve loss of secondary heat sink). This type of action has known consequences, and while they are better than core melt outcomes, they are worse than complete recovery. The crew is aware of this and is likely to hesitate before taking such an action-to wait for certainty to increase. In the process of planning, the decision maker is likely to look for alternatives to actions that will require negative consequences.

Cognitive Limitations and Biases. As with the other major knowledge-based processes, planning places a significant burden on the decision maker's representation. In the absence of an existing response plan, the representation must be used to identify a method for achieving some goal and evaluating that method in detail. Much of this must be done through a mental simulation of major components of the plant. Thus, an effective representation must be comprehensive, showing important connections between components and systems; it must be detailed so that sequencing of control actions can be carefully planned; and it must be flexible so that when standard methods are unavailable, unfamiliar methods can be created.

Therefore, the cognitive limitations to be most concerned about are representational limits. Few humans can develop and maintain representations of complex systems well enough to support tasks such as those described here. Typically, separate 
representations for different plant systems or components will be used to evaluate a plan that can affect several systems. When no integrated representation exists, there is a danger of missing inconsistencies or incongruities in the plan.

There are likely to be incorrect information and misconceptions in the representation, as well. Incorrect information may concern the ways in which the plant can be reconfigured or knowledge about system relations. Misconceptions may exist in the decision maker's understanding of a process or a system's function. These inaccuracies can lead to the development of a response plan that is faulted in some way-e.g., it does not address the goal directly, its criteria for application are not met, it does not fully specify the precautions that need to be taken before an action is executed. Misconceptions can also exist in the decision maker's understanding of HLAs.

When the decision maker becomes overwhelmed by the complexity of the task, he will not stop working on the task; he will continue to plan using whatever representation is available. Thus, the planning may be done with a simplified and inaccurate representation. Or, planning may focus on the more salient components of the plant and neglect others. In some way, detail and accuracy will be lost.

Cognitive Skills - Individuals. At the highest level of cognitive skill analysis, four skills have been identified: the ability to identify appropriate existing response plans, the ability to formulate a response plan, the ability to evaluate a response plan, and the ability to determine an action sequence. The following describes each of these high-level cognitive skills and lists the lower-level knowledge and skills that are likely to be critical.

1. Ability to identify appropriate existing response plans. When the highest priority goal is a goal that has been anticipated by the plant designers, the decision maker should select the most appropriate response plan from the set of procedures and SAMG. Because plant conditions can change drastically during a severe accident (e.g., a system fails and becomes unavailable for recovery), there may be some difficulty in selecting a response plan that can be implemented. The selection process, therefore, becomes complicated by this analysis. Further, each response plan must be evaluated to determine whether sufficient time exists, all needed equipment are available, and the potential side effects or consequences of the plan will not place other goals in jeopardy.

Lower-level knowledge and skills are:

- Knowledge of the functions (in terms of goals) of EOPs .

- Knowledge of the functions (in terms of goals) of SAMG HLAs.

- Knowledge of transition criteria between EOPs and SAMG.

- Ability to identify limits of EOPs (i.e., although EOPs may indicate actions, the actions are limited and will never lead to recovery).

- Knowledge of criteria (critical parameter values, systems that must be available) that must be met to apply HLAs successfully.

- Ability to select a plan that can be implemented and achieve goal(s).

2. Ability to formulate response plan. Existing response plans, which are rule-based methods to achieve goals, can fail for one of three reasons. First, although the goal is familiar, the standard methods to achieve the goal may be unavailable, and a new method may be required. Second, there may be a need to consider multiple familiar goals, each of which has an associated set of methods. The task then becomes one of formulating a new method out of the existing methods to achieve the multiple goals. The final failure occurs when the goal is not addressed at all in the existing response plans, and therefore, no methods have been developed for that goal. Again, the decision maker is required to formulate a new method as a response plan. The key to this skill is a detailed knowledge of the plant components and systems that allows one to identify ways in they can serve a previously unassociated function.

Lower-level knowledge and skills are:

- Knowledge of plant components and systems.

- Knowledge of alternative (deeper in terms of defense in depth) safety systems.

- Ability to mentally configure plant components and systems to achieve a function that is not typically achieved in that way.

3. Ability to evaluate response plan. The decision maker must consider the plan in depth to determine its consequences in relation to other components and systems. This analysis considers not only how to 
execute the plan successfully; potential consequences of the plan's failure and critical contingencies should also be considered.

Lower-level knowledge and skills are:

- Knowledge of critical physical phenomena during severe accident progression.

- Knowledge of plant system interconnections and relations.

- Ability to anticipate the phenomenological consequences of control actions.

- Ability to mentally simulate progression of response plan to anticipate: potential conflicts with other goals, consequences of success, consequences of partial success, and consequences of failure.

- Ability to identify contingencies if plan fails or plant state changes drastically.

4. Ability to determine action sequence. A plan has been formulated and evaluated or an HLA has been selected from the SAMG. It is possible that this planning, while needing to consider specifically which components and systems will be employed to achieve the goal, will lack specifics in terms of the sequencing and coordination of control actions. These details are addressed here.

Lower-level knowledge and skills are:

- Knowledge of specific plant systems required for implementing a plan or procedure.

- Ability to specify priority and sequencing of control actions.

Cognitive Skills - Team. As was the case in other processes when knowledge-based decision making is required, the primary decision maker has limited resources for the difficult cognitive tasks required, and needs support from other crew members. The best chance for developing an effective plan occurs when the resources of the entire team are used. It is especially critical to share information with the crew at this time. The response plan and its associated goal are largely going to determine the control actions that crew members will be asked to take. Their performance will be greatly enhanced if they have an understanding of the reasons behind their actions. Further, as the event progresses, they are also more likely to report information about the appropriateness or success of the plan. For example, if plant

conditions change locally in a dramatic way, the crew members may be able to inform the decision maker that the plan is no longer feasible.

1. Ability to provide team input to planning process. There needs to be a method or a technology that supports the crew in sharing information concerning the response plan and its goal.

Lower-level knowledge and skills are:

- Ability (decision maker) to communicate intermediate results of planning to crew members.

\subsubsection{Control}

When a plan has been selected or developed, the decision maker is then responsible for managing the timely and accurate execution of the plan. As in other cases, the use of existing procedures simplifies the process. However, when a detailed response plan is not available, there may be a need for setting priorities for control actions, allocating human and machine resources to tasks, and coordinating activities in different locations. Figure 4-5e shows that this process has been split into two components:

- Prioritize /allocate / coordinate actions

- Execute action sequence

Situational Factors. Standard emergency procedures are typically thoroughly practiced and tested. Crew members know their roles, and there is seldom a need to coordinate with the TSC. The procedures, or sets of control actions, that are executed in a severe accident setting, however, may be less routine for several reasons. First, due to the simulation model's limitations in simulating beyond-design-basis events, it is possible that crews will be unable to train on all aspects of the SAMG. Thus, this guidance is less likely to be detailed and thoroughly tested. Operators may discover ambiguities and under-specified direction. Second, crew members may be less familiar with their roles and need for coordination when using the SAMG. There may be a greater need for coordination with technicians and maintenance personnel if unusual plant configurations are required. Further, some confusion may be added by the increase in the number of people in or around the control room that is common with accident situations. All these human resources need to be managed. 
When the TSC is involved in delivering guidance or sets of control actions to the control room or other control areas, communication becomes a concern. The level of noise and activity can be an obstacle to clear communication. Some communications can require multiple links, which also increases the opportunity for error. In some fields that have little face-to-face communication and rely on electronic links, such as air traffic control, a precise and standardized language has been developed to facilitate communication. This kind of rigor can reduce communication errors in a noisy and sometimes ambiguous environment.

In prior discussions of the planning process, some of the pressures on the decision maker that push in opposite directions--both toward acting quickly to begin mitigation and toward waiting to reduce uncertainty--were described. These pressures will be present for the operators involved in execution as well. The uncertainty of risk associated with certain SAM actions may be especially salient, and therefore, operators may feel compelled to wait for more complete information. That is, HLAs and TSCdeveloped guidance may produce unfamiliar methods that may be seen by control room personnel as risky or last-resort actions.

Cognitive Limitations and Biases. Operators and technicians make errors when executing control actions. They push the wrong button, omit steps, fail to anticipate the appropriate timing for an action, etc. Many of these errors can be attributed to attentional and working memory limitations. One example of a working memory failure is a failure of prospective memory. This is the case where the operators is told to do something in the near future--the action cannot be executed immediately, but the operator should come back to it soon. When operators are very busy, these types of items get pushed out of working memory by other events, and the task is forgotten.

Certain errors of execution are referred to as "slips." One common type of slip occurs when attention is captured by something familiar that is unrelated to the task at hand. An example is the man who walks into his bathroom to retrieve a book left there. Upon reaching the bathroom, he goes to the sink to brush his teeth and wash his face. The familiar associations with the bathroom (preparing for bed) "capture" his attention, and his real intention is lost.
There are a number of attentional and memory slips (see Reason, 1990) that can lead to simple errors of execution. These error types are often contrasted to errors of intention, where the operator does not execute incorrectly but has the wrong goal in mind. That is, he correctly does the incorrect action because he was unable to determine the correct goal. Errors of intent are more central to the cognitive skills addressed in this project.

Cognitive Skills - Individuals. At this point in the model, people other than the primary decision maker begin to take larger roles. Even though a response plan may be developed by TSC staff, it is assumed that the execution of the plan will be managed by control room personnel. Cognitive skills are most critical in the case where control actions are directed or guided by something less than formal procedures.

At the highest level of cognitive skill analysis for this process, two skills have been identified: the ability to manage the execution of a response plan and the ability to execute control actions. The following describes each of these high-level cognitive skills and lists the lower-level knowledge and skills that are likely to be critical.

1. Ability to manage the execution of a response plan. This component of performance involves managing the human resources required to execute a non-trivial response plan and making determinations about the allocation and timing of individual actions. This is one area where there is a clear distinction between metacognitive skills dedicated to addressing plant conditions and metacognitive skills dedicated to managing the accident response.

Lower-level knowledge and skills are:

- Ability to specify control actions in sufficient detail for control personnel.

- Ability to communicate an understanding of the response plan and its goal to all control personnel.

- Ability to prioritize control actions when implementation resources are insufficient.

- Ability to determine the need to regulate automated control systems.

- Ability to coordinate all required personnel.

2. Ability to execute control actions. Operators and technicians may be required to carry out a set of 
control actions that may be unrehearsed or completely unfamiliar. The set of actions will be provided to control personnel, but difficulties may arise in terms of when and how much for each action. Skilled performance involves making the correct control action--including both simple, discrete actions, as well as complex, continuous actions-and the coordination of multiple individuals in control. Another consideration are control actions that have been specified by a decision maker but cannot be implemented by an operator. For example, a local control board failure may prohibit the execution of an action as specified. In this case, crew members must understand the intent of the action well enough to determine whether there are alternative means for achieving the same action.

Lower-level knowledge and skills are:

- Ability to determine that conditions for action have been met (e.g., account for changes in plant state if any, account for actions taken by others or by automated system).

- Ability to determine degree of action (i.e., how much).

- Ability to anticipate plant state changes and to prepare for appropriate action or appropriate regulation of action.

- Ability to compensate when an action cannot be executed exactly as specified.

Cognitive Skills - Team. Because there is a chance that the control actions will be less well tested, coordination in execution can become a more important issue. There may also be a greater need for coordination with personnel who are operating outside of the control room, especially if any reconfiguration of plant systems is required.

1. Ability to coordinate related control actions. Lower-level knowledge and skills are:

- Ability to coordinate actions with others.

\subsubsection{Feedback}

The feedback process focuses on the information that is gained from execution of the response plan. Figure 4-5f shows that the performance model identifies information at three levels:

- Monitor actions

- Monitor parameters / processes / functions
- Monitor goal achievement / plan

First, the operators receive feedback from the controls that the control action was executed successfully (e.g., the switch is in a new position). Second, crew members should see changes in parameter values or in indications of plant functions that are the result of the control action (e.g., flow is reduced, pressure decreases). At the highest level, the crew is provided information about whether the control actions are supporting goal achievement. This component also provides an opportunity to evaluate the response plan. When plant state or the decision maker's understanding of plant state shift dramatically, he needs to be able to modify or abandon the plan.

Situational Factors. Similar to the early stages of monitoring plant state data to develop an understanding of plant state, feedback can be impaired by difficulties with plant state indications. As a severe accident progresses, indicators may fail or become unreliable. In some cases, the optimal feedback information is not well instrumented or enters an uncommon range where discriminability is poor. All of these problems can reduce the crew's ability to determine whether the control actions are having the intended effects on plant state. Also, there are likely to be data that are less valid or transitory indications of plant state. For example, in PWRs there are transitory shrink and swell effects that can mask more enduring changes in plant state.

Cognitive Limitations and Biases. The primary consideration in this process is limitations on monitoring at the level of goal achievement and the response plan. Limited working memory and attention are likely to be devoted to lower-level monitoring and management of the response plan in addition to other necessary tasks. Periodic evaluation of the response plan, although critical, is likely to be lost in the competition for resources.

A second concern is with confirmation bias, which was also discussed under Interpret Current State. A confirmation bias suggests that decision makers will seek out information confirming that the chosen plan is correct but will not seek information that can disconfirm the chosen plan or confirm an alternate plan. Disconfirming information can be ignored or discounted, which in turn can lead to fixation. That is, after the knowledge-based work is completed one time--an interpretation is made, a goal is identified, 
and a response plan is developed-there is little sensitivity to new data. The decision maker becomes fixated on a single representation of plant state and disregards cues suggesting another interpretation.

The reverse problem, an oversensitive criterion for shifting plans, can occur as well, although it is much less likely. In this case, the decision maker sees each new indication as more salient than the last and modifies his interpretation to focus on that symptom. This approach results in fairly rapid changes in the goal and response plan as the event progresses, with no overarching interpretation of the state of the full plant.

Cognitive Skills - Individuals. Skilled performance in this element of decision making requires an ability to anticipate the effects that control actions should have on the plant and to be able to isolate valid indications of those effects. At the highest level of cognitive skill analysis for this process, two skills have been identified: the ability to use plant state data to determine that control actions are having desired effect and the ability to evaluate appropriateness of the response plan. The following describes each of these high-level cognitive skills and lists the lowerlevel knowledge and skills that are likely to be critical.

\section{Ability to use plant state data to determine that control} actions are having the desired effect. Determining that a control action was executed successfully is straightforward and direct. However, as the desired effect moves higher in the description of plant state data (from changes in parameters values to changes in plant processes), the monitoring task can become more difficult.

Lower-level knowledge and skills are:

- Ability to determine that control action was executed successfully (e.g., valve closed).

- Ability to read instruments accurately.

- Knowledge of a control action's expected effect on parameter.

- Ability to determine that control action produced desired effect on key parameters.

- Ability to determine progress toward response plan goal.

2. Ability to evaluate appropriateness of response plan. As the event progresses, the decision maker gains information from the effects of control actions that were taken and from changes in parameters that reflect the event's evolution. When there is a formal procedure (from the EOP or the SAMG), it may make explicit the changes that should occur in plant state as the plan is executed. The decision maker's representation of plant state must change to accommodate new information. Some periodic checking is required, then, to determine whether the plan being implemented is still appropriate. Questions are raised: Is the event still progressing as believed? Is the goal selected still the most appropriate? Is the response plan still the most likely to succeed? The decision maker must evaluate new evidence as it appears and must be willing to modify or abandon inappropriate response plans.

Lower-level knowledge and skills are:

- Knowledge of the changes that should occur in plant parameters as the plan proceeds.

- Knowledge of the changes in conditions that change the appropriateness of a plan.

- Ability to re-evaluate the response plan in the face of changing conditions.

\subsubsection{Summary}

The preceding sections, by stepping through the performance model, have attempted to characterize the knowledge and skills required to perform at a high skill level and to eliminate or mitigate performance problems (cognitive limitations, biases, etc.). Table 5-1 provides a summary of the high-level cognitive skill descriptions identified above. Note that these descriptions correspond to the highest level (least detailed) of cognitive skill description (see Section 2.1.4). That is, specific domain knowledge and specific cognitive processes have not beenincluded. However, an attempt was made to suggest the types of knowledge and types of decisions that need to be made within the more general categories of cognitive skills. For example, "knowledge of environmental limitations on instrumentation" and "ability to identify the conditions under which backup indications are needed" were identified under the heading "ability to determine accurate indications of plant state." For a more detailed specification of cognitive skills, a different form of cognitive task analysis could be applied to identify specific knowledge and the rules that can be used to decide that back-up indications are needed. Thus, the list of cognitive skills developed is not a sufficient specification of cognitive skills for developing 
Table 5-1. Summary of high-level cognitive skills

\section{Monitor /Detect}

1. Determine accurate indications of plant state

2. Integrate plant state indications

3. Identify meaningful events

Team:

1. Communicate important plant state indications to the decision maker.

\section{Interpret Current State}

1. Make inferences about current plant state

2. Determine expected influences and relevant data

3. Recognize links to existing procedures and SAMG

Team:

1. Provide team input to interpretation process

\section{Determine Implications}

1. Use mental representation to simulate event's progression and identify goals.

2. Determine goal priorities.

3. Recognize links to existing procedures and SAMG.

Team:

1. Provide team input to goal identification / priority setting process.

Plan

1. Identify appropriate existing response plans.

2. Formulate response plan.

3. Evaluate response plan.

4. Determine action sequence.

Team:

1. Provide team input to planning process.

\section{Control}

1. Manage the execution of a response plan.

2. Execute control actions.

Team:

1. Coordinate related control actions.

\section{Feedback}

1. Use plant state data to determine that control actions are having desired effect.

2. Evaluate appropriateness of response plan. 
detailed training materials. However, this list does identify the general cognitive skill categories required for decision making.

It is also important to emphasize that these cognitive skills can benefit performance even when rule-based decision making is used. As was noted, given that SAMGs are being developed, it is very unlikely that control room operators or TSC staff will be required to perform in a purely knowledge-based mode.

However, as was described in Section 3.1, cognitive skills support rule-based performance in a number of ways: develop expectations, support confirmation and disconfirmation of hypotheses about plant state, aid in interpreting procedures, aid in communicating intent, aid in evaluating that the selected plan is succeeding, etc. Hence, this analysis illustrates how pervasive cognitive skills are in all aspects of decision making--from detection to execution. Further, early indications are that SAM will require some elements of knowledge-based decision making (e.g., evaluating strategies and selecting the one that best fits the current situation).

One area in which cognitive skills are clearly critical is in the support of crew coordination. Especially during SAM decision making, crew members are distributed and have access to different information. Cognitive skills make possible effective communication between crew members and increase the likelihood that critical information gets to the decision maker. If the crew can work from the same deeper understanding of the situation--that is, an accurate mental representation of the event, a recognition of the highest priority goal, and a clear idea of the functional intent of the plan or procedure for achieving that goal--crew performance is enhanced in several ways. First, resources available to determine plant state are increased. Incorrect assumptions made by the decision maker can be confirmed or disconfirmed by crew members as they carry out their duties. The crew has greater access to plant state data than does a single person. Second, any preliminary evaluations--of plans or HLAs, of mitigation options, etc.--can benefit from inputs from the entire crew. The burden for thinking through all potential side effects is distributed. Third, when responsibilities are passed among crew members, the new responsible agent understands the intent of his actions. When this is true, actions can adapt to changing circumstances (e.g., a component becomes unavailable and a new method must be devised).
Fourth, the burden for monitoring the plan is distributed. The entire crew can become involved in identifying indications that allow evaluation of plan execution.

Thus, crew- or team-level cognitive skills support communication and distribute the burden of decision making to the entire crew. Crew members need to have the cognitive foundation as well as an open communication channel in order to provide meaningful inputs to the decision maker. The concept of "shared mental model" has been used to describe an entire crew that shares the same deep understanding of the situation (Cannon-Bowers, Salas, \& Converse, 1992).

\subsection{Identification of Cognitive Skills from an Analysis of SAM Scenarios}

The analysis described in the previous section provided one technique for identifying the cognitive skills required for decision making (i.e., via a performance model). A second, more empirical, approach was to be employed to confirm the results of the analytic approach. This second technique would present SAM scenarios to utility crews for a table-top analysis. This exercise would provide an opportunity to identify potential critical SAM tasks and then identify the cognitive skills required for those types of tasks. It would also provide a means for confirming or disconfirming the performance model created for SAM.

Twelve SAM scenarios (six PWR and six BWR) were developed but were not used for the table-top analysis because of changes in the program. However, the development of these scenarios did provide insights about cognitive skills.

A description of how scenarios were developed, the intended use of the scenarios, the value of these scenarios outside of the intended context, and the scenarios themselves can be found in Appendix A (Methodology), Appendix B (PWR scenarios), and Appendix C (BWR scenarios).

The SAM scenarios create situations in which the following are generally true: 
- Standard means for mitigating problems are removed or impaired

- There are potentially multiple, inter-dependent threats to plant safety

- The phenomena tied to the event are less familiar or less well understood

Although the table-top analysis was not completed, a simpler test of the performance model was conducted. Each scenario was linked back to the performance model by determining the degree to which the activities suggested by the scenario were accounted for by the elements of the model (see scenarios in Appendices $B$ and $C$ for details). If anticipated scenario activities could not be accounted for by the model, it could be declared incomplete. However, this test revealed no significant flaws in the model.

Primarily, the scenarios emphasized three broad areas of the model: interpret current state, determine implications, and plan. As Figures 4-8 and 4-9 show, these are the elements of the model most strongly tied to knowledge-based decision making. However, the SAM scenarios keep the panel tied to procedural guidance and rule-based performance, and cognitive skills are typically required to judge or evaluate advice from the EOPs (or some other source). Thus, similar to the example in Section 3.1, the primary role of cognitive skills is to use rule-based guidance wisely, not to develop procedures from an unguided reasoning process.

These scenarios confirm the importance of the following cognitive skill descriptions:

Interpret Current State:

- Make inferences about current plant state.

- Determine expected influences and relevant data.

Determine Implications:

- Use mental representation to simulate event's progression and identify goals.

- Determine goal priorities.

Plan:

- Evaluate response plan.

- Determine action sequence.

Control:

- Execute control actions (the timing of actions, metering of actions, etc.)
Thus, while the SAM scenarios were not used for the table-top analysis, they did aid in confirming assumptions underlying the performance model. Further, they provided tangible illustrations of the types of decision-making activities that may be required in SAM. 


\section{Chapter 6 Cognitive Skill Training: Principles and Techniques}

Chapter 5 identified a set of cognitive skills that are likely to be important to SAM decision making (see Table 5-1 for a summary). A primary goal of this report is to identify training techniques that address cognitive skills effectively. This chapter addresses this goal in two ways: First, Section 6.1 offers a set of principles for cognitive skill training that establish a broad context for effective training of cognitive skills. Second, Section 6.2 introduces 19 techniques for training cognitive skills or facilitating the training of cognitive skills, applying both to individuals and teams. Each technique is described, and additional information on each technique (research basis, examples, etc.) can be found in Appendix D.

Although it was not within the scope of this report to design an ideal approach for training SAM cognitive skills, this chapter does include some analysis of the training techniques introduced in Section 6.2. First, Section 6.3 proposes a scheme for mapping them to the set of SAM cognitive skills. Then, as one means for summarizing the 19 training techniques, Section 6.4 identifies a set of five general characteristics that are critical to effective training of cognitive skills. Each of the 19 techniques is then compared to these characteristics. Finally, Section 6.5 describes alternatives to training and identifies the SAM cognitive skills perhaps better suited to those alternatives.

\subsection{Principles of Cognitive Skill Training}

This section establishes a broad context for the effective training of cognitive skills. It is not sufficient to be concerned only with specific techniques to train cognitive skills in isolation. Complex technical skills (such as nuclear power plant operation) require the integration of cognitive skills with perceptual skills, motor skills, procedures, etc. The objective of the curriculum or training program is to ensure that these highly integrated skills are mastered. The trainingprogram designer must not only be concerned with specific techniques to train cognitive skills, but also with how cognitive skill training is integrated into the full training program.
The intent of this section is to describe the principles underlying an effective approach to training complex, cognitive skills--an approach that connects the mechanisms underlying cognitive skills with the techniques for training them. Note that the principles described here are not specific to nuclear power plant decision making. The five subsections that follow provide a foundation for structuring a training program that addresses a job in which cognitive skills play an essential role (see also, Schneider, 1985).

1. Define an explicit model of skilled performance. The discussion of cognitive skills in Chapter 2 identified a number of elements that comprise cognitive skill: knowledge, knowledge representation, rules for processing knowledge, domain goals and subgoals, and the strategies that can be used to achieve subgoals. By identifying the specifics underlying each element-i.e., specific knowledge, specific strategies, etc.--one can establish the instructional objectives, or goals, for training. In fact, the inability to develop this specification has long been a strong impediment to cognitive skill training. Until recently, cognitive skills were not described in such explicit terms, and instructional designers had difficulty defining the contents of cognitive skill training.

Cognitive task analysis techniques now exist that can be used to identify the specifics underlying cognitive skills (see Roth \& Mumaw, in press). For example, they allow one to analyze a complex decision to determine the information taken from the world, knowledge available in memory, the specific rules and principles that underlie inference making and problem solving, the subgoals that are useful for guiding the application of rules, etc. This analysis specifies a decision process in detail that can then serve as a model of skilled performance, and the training program can focus on the process of decision making in addition to the decision outcome.

Cognitive task analysis techniques include methods for analyzing the domain through a functional decomposition (e.g., Woods \& Hollnagel, 1987; Corker et al., 1986), analyzing the skills underlying expert performance (e.g., Lesgold et al., 1986; Means et al., 
1988), and developing computer-based simulations that can perform cognitive tasks (Roth, Woods, \& Pople, 1992; Kieras \& Polson, 1985). Although these methods can be resource-intensive and timeconsuming, they have proved their value in the analysis of cognitive skills.

Having an explicit model of skilled performance allows one to focus instruction more directly on the elements of cognitive skill, both in initial instruction and in instructional remediation. Remediation is aided because the model can be used to identify the underlying cause of performance errors. That is, novice performance can be cast as a degraded version of skilled performance, and remediation can be directed at the specific nature of the performance deficit.

2. Provide an effective mental representation of the task. Although complex technical jobs--such as electronics maintenance, air traffic control, NPP operations-require the integration of many different skills, the job typically revolves around a central task. In NPP operations, the central task is controlling plant systems to achieve the desired operating conditions. To a large degree, everything trainees learn serves to enhance their ability to perform that task. It is critical for trainees to understand the essence of this central task and be involved with it from the very beginning of the training program because this experience provides context for later learning.

There is substantial evidence that the acquisition of new information is most efficient when a strong context is provided. The description of cognition in Chapter 2 emphasized the primary role of knowledge-driven processes that attempt to fit existing notions of the world onto incoming information. Recall that humans rely heavily on mental representations of objects or events to make sense of the world. That is, new information is interpreted in the context of an existing mental representation. Thus, by establishing a coherent representation of the central task, new information can be interpreted in the context of the requirements for performing that task, and learning is facilitated.

3. Train component skills and knowledge in a jobrelevant context. This guideline is an extension of the last guideline. Training inevitably will require the presentation of new knowledge (e.g., theory, control room operations, policy) and component skills (e.g., measurement techniques, finely tuned control actions). While these component skills and knowledge may be introduced in a context outside of the job setting (e.g., classroom), they should always be integrated back into the job context. Moreover, factual knowledge must eventually take a procedural form, and therefore, it needs to be integrated into task performance. As stated above, isolated factual knowledge and individual skills can be given meaning by associating them with their function in the context of the job. The training program should continue to motivate learning by identifying the role of the new knowledge in the context of the job (What's the value of learning this? How will it aid me in improving my skills?). And, the training program should continue to support integration of new knowledge and skills into the larger, but more meaningful, task.

4. Manage trainees' mental resources. Both the description of cognitive skill (Chapter 2) and the analysis of decision-making skills (Chapter 5) identified limitations in human attention and memory as an important issue. There are strong constraints on information processing--limits in attention, limits on working memory space, limits on computation, limits on representation building, etc. One objective of a training program should be to manage the resource requirements placed on trainees. Learning can become very inefficient when trainees are overwhelmed by large demands on attention or working memory.

Generally speaking, there are two techniques for managing mental resource requirements for trainees: providing support in task performance and reducing task performance requirements. The first technique uses a second agent, a machine or another human, to assume some of the burden of task performance so that the trainee can focus on a subset of the task elements. The second technique for reducing task performance requirements is to focus training on some subset of the task elements. This technique is often referred to as part-task training (PTT). The difficult issue in the development of PTTs is how to isolate task components in a meaningful way.

PTTs and lower-fidelity simulations can be effective early in training for presenting task elements that are more efficiently learned in isolation. To be true to other instructional principles, however, it is critical to return periodically to some form of the actual 
integrated task to provide context for the component skills. Gradually, as component skills are mastered, training on the integration of skills allows the dedication of mental resources to higher-level task demands, such as metacognitive skills.

\section{Structure simulation problems to emphasize} cognitive complexities. Simulation is a very powerful training tool. Much of the instructional value of simulation is that the task environment can be structured carefully to focus a trainee on a specific learning experience. One key to the effective use of simulation is to consider carefully the range of experiences provided to trainees and to include several descriptions of complexity. One form of complexity is the complexity captured by procedures. There are a number of quantitative attributes used by the NRC (see NUREG 1021) to evaluate scenario complexity. These include number of crew critical tasks, number of EOPs entered, number of major events, etc. Also included here are attributes such as number of component failures and number of instrument malfunctions. While these quantitative attributes clearly add complexity to a scenario, there are also important cognitive elements in nuclear power plant operations that create difficulties for operators and are just as real as the difficulties that come out of the procedures. Mumaw and Roth (1992) have catalogued situations that create these complexities, which include:

- Situations that remove or obscure the usual evidence or critical evidence. For example, a loss of off-site power prior to a steam generator tube rupture can obscure the initial indications of the tube rupture.

- Situations in which important indications can be incorrectly "rationalized away." That is, indications that should be diagnostic may be attributed to a consequence of a known but less significant failure or of an automatic action (e.g., shrink and swell).

- Situations in which multiple faults mask each other.

- Situations in which faults produce effects at a distance. For example, in some interfacing system loss of coolant accidents, indications can appear in multiple systems that are not commonly associated.

- Situations that deviate from operator assumptions or expectations. For example, a safety system may fail to respond as demanded, or human errors occur in carrying out control actions to mitigate a component or system failure.

The SAM scenarios, which are found in Appendices B and $C$, also identify qualitative attributes that contribute to complexity. Examples are the need to deal with goal trade-offs (e.g., core cooling vs subcriticality) and the need to anticipate the consequences of a fairly novel phenomenon.

Simulation scenarios should not be only a means to expose operators to a set of experiences that are otherwise expensive or dangerous in the actual setting. Simulation scenarios should be both systematic and explicit in their presentation of the sources of complexity that can be found in operations. By making the application of complexity to scenarios systematic, the instructional designer can build a progression of scenario complexity that is tied to the progression of trainee skills and knowledge.

Specification of a training program. These five principles are effective for facilitating learning because they reflect primary mechanisms underlying cognition. In Chapter 2, the critical role of knowledge representation and the importance of knowledgedriven processes, which use mental structures in LTM to direct attention and guide the selection of information from the world was described. Because of the central role of knowledge-driven processes in acquiring knowledge, the training program should provide an effective mental representation of the task, and component skills and knowledge should be trained in a task context. Another fundamental aspect of human cognition are the limitations on information processing, both in attention and working memory. These limitations require that the training program take measures to manage the requirement for trainees' mental resources. Otherwise, trainees can become overwhelmed by information-processing demands, resulting in a very inefficient instructional setting. ' Finally, the major elements of cognitive skill identified above allow one to describe cognitive skill in more detail and also specify important characteristics of the domain. This specification provides more concrete objectives for cognitive skill training and aids in the development and sequencing of simulation scenarios.

These five principles identify qualities that should be woven into a training program in which cognitive 
skill training is central. They lead one to a general set of training-program objectives:

1. Identify the cognitive skills required for skilled performance and use that analysis to establish training objectives and provide remediation to trainees. For example, use a skilled operator's performance to demonstrate the use of special knowledge in decision making.

2. Within the first days of training someone for a new position, provide a means for the trainee to acquire an understanding of the central task. For example, have trainees observe actual performance in the control room and get them involved in a low-physical-fidelity simulation of nuclear power plant control that reveals the major job functions and requirements.

3. Maintain a job context for the purposes of motivating and integrating the acquisition of knowledge and component skills. For example, use a low- or medium-fidelity simulation for periodic practice on simple control scenarios that show the utility of theory or other factual information.

4. Early in the training program, identify job elements that can be isolated and trained on PTTs or other stand-alone trainers as a means to manage mental resources. Training these skills on something other than a high-fidelity simulator is easier, and later training on higher-level skills is facilitated. For example, train personnel on the fast and accurate use of a computer-based or paper-based decision aid.

5. Keep training device interfaces simple in early training, and add complexity later as the interface takes on higher levels of physical fidelity. For example, in early simulations, use a touch screen to execute control actions-perhaps a single touch achieves a realignment to a new source of coolant. In later simulations that have higher physical fidelity, use a mockup or simulation of the actual control panel to execute control actions.

6. Develop a series of simulation exercises such that each exercise focuses on a small set of individual skills, there is a progression of skills over the series, and the full range of task complexity is introduced over the series.

\subsection{Techniques for Training Cognitive Skills}

Numerous techniques and methods have been developed for training cognitive skills. This section, groups techniques based on the goals of training. Note that these groups or classes are artificial distinctions used for the purpose of exposition. The classes include the following:

- Training to teach knowledge

- Training to teach knowledge representation

- Training to teach rules applied to decision making

- Training to teach strategies, goals, and subgoals

- Training for management of mental resources

- Training a decision-making process

- Training team skills

Table 6-1 lists the full set of 19 techniques. The following sections provide general descriptions of the specific techniques that have been developed.

Appendix $D$ provides more detailed descriptions of these techniques and the research behind them. Interested readers should consult the material in Appendix D to see examples, to understand the theoretical foundations of the technique, or to identify links to broader instructional issues.

\subsubsection{Training to Teach Knowledge}

Knowledge is a critical component of cognitive skills. The complex cognitive skills required for nuclear power plant decision making are built on knowledge of thermodynamic theory, plant systems and operation, specific phenomena, plant system interconnections, the logic underlying procedures, plant-specific facts and relationships, etc. This extensive knowledge must be available-in someone's head, in a procedure, in a schematic, on a CRT, etc.--to support decision-making. The primary concern here is with the knowledge that needs to be in someone's head. Teaching knowledge is typically perceived as straightforward and uncomplicated. However, while trainees can often learn knowledge quickly, two types of knowledge failures can occur: knowledge is not tied to task performance (i.e., it is inert), and knowledge is forgotten when it is needed. Training techniques exist to address each of these potential failures. 
Table 6-1. The set of 19 training techniques.

\section{Training to Teach Knowledge}

1. Use a Job or Functional Context

2. Use Overlearning

3. Use Distributed Practice

4. Use Contextual Variety

5. Use Cooperative Learning and Peer Teaching

6. Use Accelerated Learning Programs

Training to Teach Knowledge Representation

7. Train Perceptual Patterns

8. Train Mental Models

Training to Teach Rules Applied to Decision Making

9. Eliminate Buggy Rules

10. Train Production Rules

Training to Teach Strategies, Goals, and Subgoals

11. Use Cognitive Apprenticeship

12. Use Coached Practice Environments

13. Use Planning-Support Environments

Training for Management of Mental Resources

14. Reduce the Need for Mental Resources

15. Eliminate Inefficient Strategies

Training a Decision-Making Process

16. Train Formal Decision-Making Procedures

17. Reduce Decision-Making Biases

Training Team Skills

18. Use a Behavior-Based Aircrew Coordination Training Technique

19. Develop Shared Mental Models 
Inert knowledge (Bereiter \& Scardamalia, 1985) is knowledge that can be used in the context of a classroom or testing situation (e.g., repeating back facts). However, even though the trainee can demonstrate the availability of this knowledge in some settings, the knowledge is not tied to its use in the context of task performance. As Brown and Palincsar (1989) note, inert knowledge "fails to become part of a usable store of knowledge (p. 394)." As was stated in Section 6.1, a primary rule for training knowledge is to establish a job context and a meaningful representation of the task in which the knowledge will be employed. When training is done in a job context, knowledge becomes tied to the job context, or in cognitive terms, the knowledge becomes part of a cognitive procedure.

There are six training techniques available for training knowledge to prevent these two types of knowledge failures.

1. Use a job or functional context. Knowledge should be presented in the context of its use on the job. That is, trainees need to be involved in realistic job tasks when learning new knowledge. The context of the job provides a structure on which to hang new knowledge and creates a link between the knowledge and its use, therefore reducing the likelihood of producing inert knowledge. The knowledge becomes part of the skill and is better retained.

There may be cases in which knowledge is less easily tied to its application for task performance. That is, the curriculum may specify a need to teach knowledge--perhaps lists, associations, simple procedures, facts--that trainees must master and retain until it is required for job performance. The following techniques provide alternatives to make this type of learning more efficient and increase retention.

2. Use overlearning. One technique to enhance longterm retention is called overlearning. Overlearning refers to practice that extends beyond the achievement of some level of mastery. For example, a training objective might (somewhat arbitrarily) define mastery of a task as the ability to complete the task without performance aids (e.g., a written procedure) in less than 2 minutes within some level of tolerance. Practice on this task during initial training may be terminated as soon as this criterion has been achieved. Under these conditions, learning level will fall off over time after practice has ended (see Figure D-1 in Appendix D). Overlearning, however, would advocate practice beyond the mastery level.

Overlearning, sometimes called supplementary practice or post-mastery learning, can be specified in a number of ways-additional time, additional practice trials, etc. One common method is to express it in terms of the practice or training time required to reach mastery. For example, a $50 \%$ overlearning criterion would require each trainee to practice an additional three hours on a skill that required six hours of practice to reach mastery. Likewise, a 100\% overlearning criterion would double the practice time required to reach mastery.

3. Use distributed practice. While the number of practice trials or training time is strongly related to the level of learning, retention also depends on the distribution of practice trials across time. For a fixed amount of practice, long-term retention can be enhanced by spacing practice instead of massing all practice into a single session. For example, four hours of practice can be given in a single day (massed practice) or split into four sessions, each of which is one hour and separated by several days from other practice sessions (distributed or spaced practice).

4. Use contextual variety. Another technique to enhancing long-term retention of knowledge and simple skills is to vary the training setting. This technique, which introduces variety to the training context, is sometimes called contextual variety or contextual interference. Like other techniques discussed here, contextual variety hinders initial learning but enhances long-term retention. The goal of this set of techniques is to force trainees to develop a more elaborated or richer mental representation that can be accessed more easily and used more flexibly.

5. Use cooperative learning and peer teaching. Another means for getting trainees actively involved with the material is to hand the role of instructor to trainees. Cooperative learning and peer teaching are techniques that force learners to take on multiple roles in approaching the material, and they couch learning in a more comfortable social setting. In cooperative learning, the material to be learned is divided, and each member of the group becomes responsible for instruction. Various techniques that have been applied in school settings are Jigsaw (Aronson, 1978), Trainee Team Achievement Division (Slavin, 1983), 
Teams-Games-Tournaments (DeVries, Slavin, Fennessy, Edwards, \& Lombardo, 1980), Group Investigation (Sharan \& Sharan, 1976), and the Learning Together Model (Johnson \& Johnson, 1975).

6. Use accelerated learning programs. A recent review of training techniques (Druckman \& Swets, 1988 ) investigated several commercially available programs that make strong claims about enhancing learning. Examples of these "accelerated learning" programs are Suggestive Accelerative Learning and Teaching Techniques (SALTT), Suggestopedia, and Superlearning. In particular, Druckman and Swets examined the program from the SALTT organization, which prescribes a method for training and sells training services. At the heart of the SALTT method are many of the techniques described here: distributed practice, trainee involvement, elaboration on material, and frequent assessment. Also included in the method, however, are non-traditional techniques, such as the use of music to enhance learning. (Note: These commercial programs are not recommended-see the discussion in Appendix D.)

\subsubsection{Training to Teach Knowledge Representation}

An important aspect of knowledge is its organization in long-term memory. Simply having access to knowledge is insufficient for skilled performance; the information must be organized within the job context. Skilled practitioners develop methods for extracting information from the world based on the meaningful patterns that occur and based on their understanding of the functional relationships between events and objects. Thus, training in this area must focus both on teaching trainees to identify important patterns and on teaching mental models that support decision making.

Extracting meaningful patterns of information is critical in monitoring and interpreting plant state information. As stated above, because typical control room or TSC interfaces provide no support in integrating information, it is up to operators and TSC staff to identify patterns of information that are meaningful. These patterns can include trends or change rates on an indicator, changes in the sounds coming from a plant system, or feedback from a control device. Accurate and complete mental models of the plant systems and procedures are also essential for supporting the types of cognitive skills identified in Chapter 5. Decision makers need to anticipate, plan, evaluate procedures and high-level actions, and manage control execution. Each of these relies on the qualitative reasoning made possible with accurate mental models.

7. Train perceptual patterns. Several techniques have been used to train perceptual patterns. The most straightforward is to flag the relevant cues in the environment and demonstrate their value. A skilled performer who is speaking aloud as he or she performs the task can identify the information being used to guide performance. A problem arises when the pattern is difficult to perceive or difficult to describe. In these cases, other techniques have been used to guide learners. In one case (see Vidulich, Yeh, \& Schneider example in Appendix D), the critical pattern was difficult to perceive because it unfolded slowly over time. To make the pattern more salient, the time course of the event was compressed to run faster than real-time. In cases where the pattern is spatial and difficult to put into words (see the Lintern \& Roscoe example in Appendix D), several techniques have been developed to aid the trainee in identifying and learning the appropriate visual cues.

8. Mental models. To guide the development of mental models for reasoning and problem solving, White and Frederiksen (1986) have developed a technique to assist trainee development of mental models using a computer-based learning environment, called QUEST. Their technique is intended to lead trainees through a progression of models, beginning with simple models and progressing to more comprehensive and more complex models. Levels of mental model are defined, and a set of problems is defined for each level of mental model that is being trained. Early in training, trainees work with few rules or constraints and need only determine gross changes in state of components. When these problems are mastered, the learning environment presents problems that require a more complex mental model. Thus, change is motivated by the trainee's inability to solve problems.

Through a number of activities, trainees eventually transform their model. This transformation can occur through the addition or refinement of knowledge or rules, a generalization of rules, a differentiation of rules, or in some cases, a shift to an alternative model. This transformation or progression of models is supported with four types of learning activities: open-ended exploration, problem-driven learning, 
example-driven learning, and trainee-directed learning (see Appendix $D$ for examples).

At this time, because these training devices were part of research programs, they have not been widely evaluated. Initial evaluations with small groups of trainees have been very encouraging. For example, White and Frederiksen found significant

improvements in problem solving with seven trainees over a short period of training.

\subsubsection{Training to Teach Rules Applied to Decision Making}

An early phase in the development of cognitive skills is the construction of domain-specific rules.

Anderson (1983), in his description of cognitive skill acquisition, has focused on the development of condition-action pairs, called production rules, that proceduralize knowledge for the performance of specific tasks. There have been a number of analyses of simple cognitive tasks that have successfully derived a set of rules for task performance (e.g., Card, Moran, \& Newell, 1983; Kieras \& Polson, 1985). These rules can form the basis of an expert system that can solve problems or make decisions. In the SAM setting, these rules form the basis of a number of decision-making tasks-for example, setting priorities or selecting goals, planning, and the coordination of control actions.

The analysis of rules has led to two techniques to training. One technique emphasizes identifying and eliminating incorrect rules, called buggy rules, that are formed in the course of learning. The second technique uses the rules underlying the model of skilled performance to guide trainee performance as a task is learned.

\section{Identify and eliminate buggy rules. While} involved in analysis of students' errors in basic arithmetic, Brown and Burton (1978) began to document systematic errors in students' procedures. That is, they determined that errors were not simply random occurrences, but reflected the presence of incorrect rules that had been acquired in the process of learning. These rules were called buggy rules (as a reference to the "bugs" found in computer code). Brown and Burton (1978) found that teachers typically dismiss such responses as random errors, instead of systematic errors based on incorrect rules, and they developed a series of projects to aid in the identification and elimination of buggy rules.

The first training effort pursued was to develop a diagnostic tool that could aid teachers in identifying the buggy rules that students had acquired. A system called BUGGY (Brown \& Burton, 1978) presents math problems with incorrect responses and challenges the teachers to diagnose the incorrect rule that is present. It was also presented to students for the same purpose. In both cases, the instructional goal is to get evaluators to view incorrect responses as incorrect procedures that can be diagnosed and addressed. A later effort, IDEBUGGY (Burton, 1982), was an attempt to do diagnosis on-line as students solved arithmetic problems.

10. Train production rules. Anderson has extended the rule-training technique to create a number of intelligent tutoring systems (ITSs) that address LISP programming and several areas of math education. The LISP tutor, called LISPITS, has been evaluated and refined over the last 10 years (see Corbett \& Anderson, 1992). LISPITS is a tutor for supporting programming exercises. It presents an exercise that requires the trainee to develop LISP code (or a very short program).

The set of problems presented to the trainee provide opportunities to learn the set of rules used by the expert system component of the tutor, which can generate correct code for all problems. LISPITS determines at every point in code generation the set of possible trainee responses, using both correct rules and common buggy rules. This is the means by which LISPITS diagnoses errors and keeps track of the correct rules available to the trainee. Thus, the trainee's responses indicate the production rules acquired as well as any buggy rules acquired.

\subsubsection{Training to Teach Strategies, Goals, and Subgoals}

A very important subset of cognitive skills are those associated in Chapter 2 with simple task performance. Beyond applying general cognitive processes to knowledge to develop task-specific rules (or cognitive procedures) is the need to apply those rules to solving problems or making decisions. Recall that the Newell and Simon (1972) goal-oriented framework for describing performance was adopted. Specifically, a task can be analyzed into an organized collection of 
goals and subgoals that must be effected in order to accomplish the task. After the set of goals is established, the performer must then identify the specific rules that can be applied to accomplish each goal. A strategy was defined as the sequence of rules used to achieve a subgoal or goal. More generally, these elements are central to the broader activity of planning. In the SAM context, strategies, subgoals, and goals are critical elements of accident management. The skilled decision maker will know how to achieve the operational goals most relevant to the current plant state and then ensure that the procedure document is adopting that same approach.

A number of training techniques have been developed and refined over the last 15 years that focus on these elements of cognitive skill. These techniques are grouped into three categories: cognitive apprenticeship, coached practice environments, and planning-support environments.

11. Use cognitive apprenticeship. Collins, Brown, and Newman (1989) describe a technique, which they call cognitive apprenticeship, for teaching the skills of reading, writing, and mathematics to young school students. This technique, however, is applicable to any cognitive skill that requires the application of knowledge to perform complex tasks. The technique relies on developing a description of skilled performance in each task and using that description as a model for instruction. Cognitive apprenticeship reveals the processes required for reading, writing, and mathematics and supports the trainee in acquiring that process. (The specific elements of this technique are identified and defined in Appendix D.)

The specific techniques developed for reading, writing, and mathematics have been very successful in the limited evaluations that have been done. For example, the reading technique of Palincsar and Brown has increased reading comprehension scores from a $15 \%$ to an $85 \%$ accuracy level after about 20 training sessions. More important, this improved performance remained relatively stable several months after the training was completed.

\section{Use coached practice environments. A second} technique, which has been employed in several intelligent tutoring system (ITS) projects, is referred to as a learning environment (Wenger, 1987) or a coached practice environment (Lesgold et al., 1992). The intent of this technique is to immerse trainees in the problem-solving activity and to coach them opportunistically. The model of skilled performance is not presented in its entirety up-front as it is in the cognitive apprenticeship technique, but is revealed via coaching that occurs throughout instruction.

13. Use planning-support environments. In section 6.2.3 techniques were described for training computer programming skills that were focused on the correct and buggy rules underlying performance. Other techniques to programming have instead emphasized the planning and strategic aspects of programming. Two ITS projects have developed visual or graphical environments that support planning and strategy development: GIL and BRIDGE.

GIL (Reiser et al., 1992) was a direct reaction to perceived weaknesses in the LISPITS approach to tutoring LISP programming (Corbett \& Anderson, 1992). Reiser (1987) found that because the overall structure (goals, subgoals, strategies for achieving subgoals) was implicit in the instruction of LISPITS some trainees were unable to acquire that element of LISP programming. LISPITS focuses on the specific commands required at each point in the program but not on why those commands would be effective.

Thus, Reiser et al. developed a tutoring environment that could help trainees reason about the conditions in which programming commands are appropriate, the function that commands have in the program, and how they advance the programmer closer to the goal of the program.

\subsubsection{Training for Management of Mental Resources}

One of the training guidelines described in Section 6.1 concerns the importance of a trainee's mental resources. Because of limitations in human memory and attention, a training program must aid trainees by ensuring that sufficient mental resources are available-initially to increase training effectiveness, later to enhance job performance. The discussion in Section 6.1 identified two general techniques to manage mental resources during training: providing support for task performance (e.g., scaffolding techniques) and reducing task performance requirements. That section also noted that there are different types of part-task trainers that can be used to reduce task performance requirements. One of those techniques, called automaticity training, is potentially a powerful technique for reducing the need for mental resources when executing essential job tasks. 
14. Reduce the need for mental resources. As was stated in Section 6.1, there have been a number of demonstrations (see Schneider \& Detweiler, 1988) of the ability to train skills to the point where their execution requires almost no mental resources. Those skilled in reading, driving, or typing are prime examples of the benefits of automating low-level skills. When these low-level skills are automated, more important activities-e.g., sentence and paragraph comprehension in reading, route finding in driving, or text editing in typing-can be performed simultaneously and still receive the level of attention they require.

Schneider and his colleagues have been exploring training techniques that take advantage of this phenomenon and that allow trainees to perform two or more tasks simultaneously. The first element of this technique is to identify a task component that can be trained in isolation to achieve automated performance. These components must be in a class of responses that are called "consistently mapped" (see discussion in Appendix D). The second element of the technique is extensive, focused training. After the task component has been isolated, trainees are given hundreds to thousands of trials over a period of days or weeks. Over these trials, response time and requirements for attentional resources decrease. Eventually, the component is integrated back into the complete task.

15. Eliminate inefficient strategies. The last section described a technique for isolating consistently mapped task components and training them to the point where they require almost no mental resources. Early in training, this development of isolated skills can aid in reducing mental resources and preventing trainees from becoming overwhelmed. However, at some point task components must be integrated, and studies have shown that automated skills may not be integrated into a dual- or multiple-task setting without a decrement in performance. Thus, there needs to be a transition to dual-task practice after the early phases of automaticity training are complete (although Schneider and Detweiler did not determine a means for identifying that transition point easily). The more general point, however, is that skills trained in isolation may be learned in an inefficient way. That is, when there are no other task demands, inefficient use of attentional and working memory resources can occur. By practicing a skill when there are additional requirements for mental resources (i.e., a second task), a trainee can learn the most efficient strategies for sharing attention and memory.

\subsubsection{Training a Decision-Making Process}

One technique for training decision-making skills is to train personnel to apply a formal decision-making process. Ideally, this training could improve decisionmaking efficiency and skill and reduce the effects of bias in decision making. Means et al. (1993) reviewed the training studies that have applied this technique, and the techniques used are presented here although the training studies Means et al. reviewed do not seem to hold promise for the current context. The shortcomings of these techniques are tied to an inappropriate notion of decision making. Specifically, the decision-making process that has been trained is not the same process as that required by real-world tasks (see Klein, Orasanu, Calderwood, \& Zsambok, 1993). Further, for the NPP context, the role of procedures in decision making must be strongly emphasized.

\section{Train a formal procedure for decision making.} Means et al. first reviewed a set of training studies in which the subjects were trained in decision-theorybased procedures, such as multi-attribute utility (MAU) models. These procedures are prescribed for decision tasks such as selecting among a well defined set of alternatives. In everyday decision making (e.g., selecting purchases) individuals trained in formal methods seem to be unlikely to adopt them, and may be unable to adopt them if time constraints exist. In these cases, these individuals fall back on computationally simpler strategies that may lead to poorer performance than the complete formal method. This phenomenon seems much less likely, however, in the environment of nuclear power plant decision making, where formal procedures guide all actions. Certainly, decision making will be strongly determined by the decision-making structure that is imposed by the EOPs or SAMG. Perhaps the lesson to be learned from these studies (see Appendix D for details) is that, under time pressure or high stress, decision making shortcuts may occur, especially if the formal procedure requires a considerable mental burden. Because of this, the decision-making procedure given to decision makers should reduce requirements for mental computations as much as possible. 
17. Reduce decision-making biases. Means et al. (1993) also review training techniques for reducing or eliminating decision biases. Instead of training a set of procedures or a method, these programs attempt to eliminate the biases that occur naturally in human decision making. Some studies (e.g., Lichtenstein \& Fischoff, 1980; Tolcott, Marvin, \& Bresnick, 1989) did show modest reductions in decision biases after training. Other studies (e.g., Bukszar \& Connolly, 1988) were unable to overcome biases with training. However, the larger problem, even in cases where training reduced biases, was achieving any generalization or transfer of training to other decisionmaking situations. Thus, it seems that any training to reduce or eliminate decision-making biases will have to focus on the biases specific to a decision-making task. Training that attempts to provide a general sensitivity to decision-making bias is not likely to be effective.

\subsubsection{Training Team Skills}

It is critical to consider training techniques that address team skills as well as those that address individual skills since teams, or crews, are essential to NPP decision making and accident management. As descriptions of skilled teams have developed (see discussion in Appendix D), a training technique has also evolved to address team skills. This technique was first developed for aircrews to address the widespread perception that an absence of crew skills was a major contributor to a number of fatal mishaps (Foushee \& Helmreich, 1988). The program was initially known as cockpit resource management (CRM), or more generally, as aircrew coordination training (ACT). The most recent and most complete form of this technique, a behavior-based ACT technique, was developed at the Naval Training System Center (see Prince, Chidester, Bowers, \& Cannon-Bowers, 1992). A description of this technique follows:

\section{Employ a behavior-based ACT technique.} According to Prince et al. (1992), the general ACT technique can have multiple phases: awareness, practice and feedback, and reinforcement. In the awareness phase, seminars and group exercises are used to present the basic concepts of team performance. In many cases, these activities have been borrowed from management courses, and the topics covered include communication, decision making, workload management, management styles, and leader and subordinate responsibilities. This phase rarely provides skill practice in an operational environment.

The practice and feedback phase is best exemplified by the line-oriented flight training program (LOFT)" (Lauber \& Foushee, 1981). LOFT uses realistic scenarios to involve the crew in a complex or difficult situation in which team skills are important. For example, a full-scope, high-fidelity aircraft simulator is used to present an aircraft system failure or weather-related problem to a full crew. The scenario typically requires the crew to execute all aspects of a mission: preparations, routine procedures, communications to individuals outside of the flight deck, etc. Crew performance is then videotaped and reviewed in a debriefing session that includes the instructor and the crew. Because these scenarios are conducted shortly after the awareness training, the crew can use them to identify areas of team performance that can be enhanced with better communication and other aspects of crew performance.

The reinforcement phase, or recurrent training phase, is a means to provide LOFT-type exercises on a recurring basis. Thus, every several months, the crew may conduct LOFT with review by an instructor. Prince et al. (1992) recommend that the most critical part of LOFT, the specific evaluation of crew performance, become a central part of the recurrent training. They emphasize that crew performance goals, in addition to individual performance goals, must become a determinant of whether each crew member maintains his certification for crew members to embrace the goals fully.

Early studies that have assessed aircrew training show that crews containing at least one member who has been involved in some form of ACT perform better than crews in which no member has had such training (Helmreich, Chidester, Foushee, Gregorich, \& Wilhelm, 1989). Prince et al. (1992) further point out that while there are no conclusive data on the effectiveness of LOFT for changing aircrew performance, there is a strong indication that the programs are accepted and liked by crew members.

A concern with the programs as they have been developed is the vagueness with which team skills are sometimes defined. For example, the team performance dimensions are not always tied to the 
same behaviors across training programs. More generally, trainers may not understand how to rate specific behaviors in terms of the team skills that are being rated. According to Helmreich and Wilhelm (1986), LOFT evaluators need to be familiar with the desired behaviors in the context of the scenario and trained to observe team behaviors and provide specific feedback. Even crew members' own ratings of their performance are inaccurate unless they understand the specific behaviors expected in the training situation. Because of these difficulties, a behavior-based technique has been developed to make explicit the types of behaviors that indicate good team skills.

19. Develop "shared mental models". The notion of a mental model was first introduced in Chapter 2. Basically, this term refers to a mental representation of a device or process that is used to generate descriptions, explain functioning, anticipate changes or progressions, etc. It integrates many types of knowledge and allows the individual to simulate a system or process mentally. Cannon-Bowers, Salas, and Converse $(1990,1992)$ have used this concept to describe high levels of communication and coordination skills in teams (see Appendix D for more detail).

Similarly, Cannon-Bowers and Salas (1990) suggest that, in order to function effectively, teams may share mental models of the task environment, of the equipment or interface, and of the team and its interactions. These various types of mental models allow crew members to share an understanding of the current state of the task, of the needs or expectations of other crew members, of the control actions that are needed, etc. This shared understanding supports coordination and communication for a number of reasons, including the following:

- Information needs can be anticipated since expectations are shared.

- Crew members can more readily adapt their performance since they understand the needs of the team.

- Coordination is more efficient since each crew member knows what information is and is not available to other crew members.

- A response to a question can be given the proper context because the intent of the question is understood.
One of the few training techniques that have been offered by Cannon-Bowers and her colleagues to support the development of shared mental models is cross-training-that is, allowing crew members to serve in a different role in order to understand the needs and demands of that role. Evaluations of various techniques are just now beginning. Thus, there are currently no good models for training programs that support the development of shared mental models.

\subsection{Mapping Specific Cognitive Skills to Training Techniques}

The cognitive skills identified in Chapter 5 are listed in Table 6-2. This table assigns each cognitive skill to a general class of training techniques. This assignment obviously captures only the general nature of each cognitive skill. Some skills could easily be assigned to several classes since the descriptions of these cognitive skills are at such a high level. More effort would be required to adapt each technique, and a full curriculum design, to the training of these skills. In the Table, each skill is preceded by an acronym that refers to the general decision-making process under which the skill was listed. Also, notice that team skills are identified explicitly.

Note that several classes are absent from Table 6-2. First, the class of techniques for teaching knowledge will be relevant to many, if not all, of the cognitive skills. The more detailed descriptions of each cognitive skill in Section 5.1 identified the types of knowledge that provides the foundation for the skill. Second, class of techniques for the management of mental resources are absent from Table 6-2. Again, this class would certainly have a critical supporting role in a full curriculum but is not the main focus of cognitive skill training. Finally, the class of techniques for training a formal decision-making process are not represented on Table 6-2 since it was treated as a means for training an inappropriate form of decision making. 
Table 6-2. Assignment of cognitive skills to training technique classes.

\section{Training to teach knowledge representation}

(listed as Performance Model Element - Cognitive Skill pair)

M/D -Identify meaningful events

ICS - Make inferences about current plant state

ICS - Determine expected influences and relevant data

DI - Use mental representation to simulate event's progression and identify goals.

$P$ - Evaluate response plan.

F - Use plant state data to determine that control actions are having desired effect.

Training to teach rules applied to decision making

(listed as Performance Model Element - Cognitive Skill pair)

$M / D$ - Determine accurate indications of plant state

$M / D$ - Integrate plant state indications

ICS - Recognize links to existing procedures and SAMG

DI - Recognize links to existing procedures and SAMG.

$P$ - Identify appropriate existing response plans.

C - Execute control actions.

Training to teach strategies, goals, and subgoals

(listed as Performance Model Element - Cognitive Skill pair)

DI - Determine goal priorities.

$P$ - Formulate response plan.

$P$ - Determine action sequence.

$C$ - Manage the execution of a response plan.

F - Evaluate appropriateness of response plan.

\section{Training team skills}

(listed as Performance Model Element - Cognitive Skill pair)

$M / D$ (Team) - Communicate important plant state indications to the decision maker.

ICS (Team) - Provide team input to interpretation process.

DI (Team) - Provide team input to goal identification / priority setting process.

$P$ (Team) - Provide team input to planning process.

$C$ (Team) - Coordinate related control actions.

\section{Legend:}

M/D - Monitor/Detect

ICS - Interpret Current State

DI - Determine Implications

$$
\begin{aligned}
& \text { P - Plan } \\
& \text { C - Control } \\
& \text { F - Feedback }
\end{aligned}
$$




\subsection{Characteristics of Effective Training of Cognitive Skills}

In terms of general characteristics that are critical to effective training of cognitive skills, at least five important qualities have emerged. These qualities run throughout the techniques described here and in the learning principles discussed in Section 6.1. They are the following:

1. Develop a model of skilled or expert performance to be used as a model and as a diagnostic aid.

2. Require trainees to become involved in evaluating their own performance (or the performance of others) using as a standard the model of skilled performance.

3. Get trainees actively engaged in the task as a setting for instruction.

4. Support performance to allow trainees to be involved (eventually) in performance of the complete task.

5. Aid trainees in managing mental workload throughout training.

First, developing a model of skilled performance through some form of cognitive task analysis is a critical input to cognitive skill training. It is important to emphasize that this analysis addresses both the knowledge required and the processes that support the application of that knowledge. Simply teaching knowledge is insufficient. The cognitive skills that are required for skilled performance serve two roles in instruction: as a model and a diagnostic aid. In some cases, a model of skilled performance is presented upfront for trainees or used to structure the instructional environment. In other cases, the model is used to generate explanations or to provide hints as learning progresses. In all cases, the tutor or instructor understands how the task should be performed and why. Moreover, this model serves as a means for assessing and remediating trainee performance. Much of the work in intelligent tutoring systems has focused on techniques for describing trainees in terms of the expert model. The instructor or tutorial system requires this diagnosis to determine the instructional focus and feedback provided to trainees as learning progresses. Note that a critical element of this diagnosis may be the identification of incorrect rules or broad misconceptions acquired by trainees. Also, it is important to acknowledge that the development of a model of skilled performance is sometimes difficult, especially in tasks such as severe accident management. In these cases, an analysis of guidance documents and other task descriptions may be used to establish the model (see Roth \& Woods, 1988; Roth \& Mumaw, in press).

A second use of the model of skilled performance is a standard for the trainee's self-monitoring and selfdiagnosis. Collins, Brown, and Newman (1989) describe the value of training trainees to be both performers and evaluators. While this may begin with the practice of critiquing others, eventually trainees learn to apply the same analysis to their own performance. The processes required for evaluation, primarily articulation and reflection in the cognitive apprenticeship model, are effective instructional mechanisms.

The third characteristic of nearly all of the effective training techniques is to involve trainees in the actual task or a simulation of the task. A simulation is preferred because of the capabilities it offers for controlling the learning environment. Using the functional context for training ensures that the knowledge acquired is tied to the procedural use of the knowledge. This is critical because of the potential for the development of inert factual knowledge. In the training techniques reviewed, trainees were engaged in a number of activities, including actual problem solving or decision making, system design, exploration, prediction making, watching example problems solved by an expert, and evaluating or critiquing the performance of others. In several cases, trainees were thrust into a simulated task environment where they could do no damage and were required to complete tasks that were well beyond their current capabilities.

A fourth characteristic of many of the training techniques described above was an emphasis on allowing trainees to be involved in the performance of the complete task. Trainees are supported initially through scaffolding or other techniques that allow them to work on single elements, but also allow them to integrate these elements into a complete whole. An important aspect of this technique is that trainees are given the opportunity to observe the metacognitive skills required for task performance. The instructor or tutor who is supporting trainees provides a control structure for accessing and executing task elements. As trainees master the lower-level elements, they can 
take on larger roles and eventually perform the entire task. The importance of involvement with the complete task does not reduce the value of part-task training. Part-task training, as indicated in previous sections, can have a role in managing mental resources. However, eventually this form of training must be integrated back into the context of the whole task.

Finally, many of the effective techniques described incorporate mechanisms for reducing the mental resource requirements of the training setting. Because cognitive skills need to be learned at various levels-execution of rules, goal achievement, and metacognitive control--it is difficult for trainees to keep track of all aspects of task performance simultaneously. Instructional effectiveness is facilitated when some of these elements can be removed in the short-term or the problem representation provides some of the structure of the expert solution. Previous sections have identified more specific methods for controlling this aspect of cognitive skill training.

At this time, there is more than adequate evidence for specifying these five characteristics as important elements of cognitive skill training. As a final summary of the 19 training techniques, Table 6-3 shows how well each technique could provide each of the five characteristics. Note that some of these techniques are more narrow-e.g., addressing only knowledge acquisition--but can still have an important role in a training program. The columns with more check marks are typically the techniques that address the task more broadly. Thus, the number of checks should not be the sole criterion for selecting a training technique.

\subsection{Cognitive Skills and Alternatives to Cognitive Skill Training}

Training is not the only solution when there are gaps between performance requirements and current skill levels. This section briefly reviews the alternatives and identifies specific SAM cognitive skills that may be better suited to alternative solutions.

One alternative for decision-making tasks are decision aids, such as guidance documents (EOPs or SAMG) and computer-based decision-support tools. In fact, the owners groups are developing or have developed an SAMG document to support severe accident management. Decision-support tools are useful ways to move off of humans and onto machines the tasks that require a large computational load. Decision aids may be used in decision making to estimate time available, to estimate the likelihood of equipment failures, or to simulate small elements of plant performance to anticipate future plant states. Several utilities have developed computer-based decisionsupport tools to aid SAM. In general, however, little more than an SAMG document is expected to be available to crews.

Another alternative are upgrades to plant sensors and interfaces. For example, new sensors, new diagnostic aids, or new interface devices could be developed to support operators and TSC staff in managing severe accidents. At this time, however, the only additional support operators and technical staff are likely to have is the SAMG document. This document has been developed first at a generic level by the owners' groups, and will then be adapted by the utilities to fit the specifics of each plant.

The use of the SAMG has the potential to remove the need for training certain skills. Specifically, cognitive skills associated with formulating or identifying appropriate response plans will probably be less critical when an effective SAMG is available. The SAMG is likely to at least provide a method for decision makers to identify a high-level action (HLA) or response strategy (or perhaps a set of useful HLAs). Decision makers are unlikely to be required to develop a response plan in a purely knowledgebased way. As stated in the discussion in Section 3.1, the crew is more likely to require cognitive skills to determine that the procedure they have been led to is appropriate, addresses the goal, can be achieved, etc. Several of the SAM scenarios (e.g., PWR-5) illustrate a need for crew members to evaluate the wisdom of the advice presented by the current EOPs. Therefore, the high-level cognitive skills (see Table 5-1) "recognize links to existing procedures and SAMG," "identify appropriate existing response plans," and "formulate response plan" should not be high priorities for training. 
1. Functional Context

2. Overlearning

3. Distributed Practice

4. Contextual Variety

5. Cooperative Learning
6. Accelerated Learning

7. Perceptual Patterns

8. Mental Models

9. Buggy Rules

10. Production Rules
11. Cognitive Apprenticeship

12. Coached Practice Environ

13. Planning Support Environ.

14. Mental Resources

15. Inefficient Strategies
16. Formal DM Procedure 17. DM Blases

18. Behavior Based ACT

19. Shared Mental Models

\begin{tabular}{|c|c|c|c|c|c|c|c|c|c|c|c|c|c|c|c|c|c|c|c|}
\hline & 1 & 2 & 3 & 4 & 5 & 6 & 7 & 8 & 9 & 10 & 11 & 12 & 13 & 14 & 15 & 16 & 17 & 18 & 19 \\
\hline $\begin{array}{l}\text { Model of } \\
\text { Skilled } \\
\text { Performance }\end{array}$ & & & & & & & 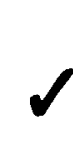 & $\checkmark$ & $\checkmark$ & $\checkmark$ & $\checkmark$ & $\checkmark$ & $\mathscr{V}$ & & & $\Omega$ & & $\checkmark$ & $\checkmark$ \\
\hline $\begin{array}{l}\text { Supports } \\
\text { Evaluation }\end{array}$ & & & & & $\checkmark$ & & $\boldsymbol{N}$ & $\boldsymbol{\Omega}$ & $\checkmark$ & $\checkmark$ & $\boldsymbol{V}$ & $\checkmark$ & $\mathscr{N}$ & & & $\checkmark$ & & $\boldsymbol{V}$ & $\checkmark$ \\
\hline $\begin{array}{l}\text { Task } \\
\text { Context }\end{array}$ & $\mathscr{V}$ & $\checkmark$ & $\mathcal{N}$ & $\checkmark$ & $\checkmark$ & $\checkmark$ & $\mathscr{V}$ & $\checkmark$ & $\checkmark$ & $\checkmark$ & $\boldsymbol{V}$ & $\checkmark$ & $\checkmark$ & & 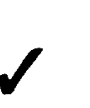 & $V$ & & $\checkmark$ & 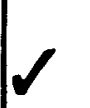 \\
\hline $\begin{array}{l}\text { Complete Task } \\
\text { Performance }\end{array}$ & & & & & & & & $\mathscr{J}$ & $\boldsymbol{\Omega}$ & $\checkmark$ & $\checkmark$ & $\boldsymbol{J}$ & $\boldsymbol{J}$ & & & $\mathscr{V}$ & & $\Omega$ & $\mathscr{V}$ \\
\hline $\begin{array}{l}\text { Manages } \\
\text { Mental } \\
\text { Workload }\end{array}$ & & & & & & & $\mathcal{J}$ & $\checkmark$ & $\boldsymbol{J}$ & $\checkmark$ & $\checkmark$ & $\checkmark$ & $\checkmark$ & $\checkmark$ & $\checkmark$ & $\mathcal{V}$ & & $\checkmark$ & $\mathscr{V}$ \\
\hline
\end{tabular}

- Numbers at top of columns refer to training techniques listed above table.

" $\checkmark$ " Indicates that the training technique could provide the characteristic.

Table 6-3. Important training characteristics for each training technique. 


\subsection{Summary and Conclusions}

Nineteen general techniques for training cognitive skills or facilitating the training of cognitive skills, applying both to individuals and teams of individuals have been described in this chapter. In summary, several points need to be made. First, a training program or an approach has not been developed that is ideally suited to the decision-making tasks characteristic of nuclear power plant operations or severe accident management. That activity was not part of the scope of this program. Instead, techniques have been identified that have the potential to be successfully applied to the types of skills required for NPP operation. Second, the classification scheme employed here to group techniques under one of seven headings is a convenient grouping scheme for description and emphasis. Many of the techniques described extend across class boundaries, and they were not developed to fit neatly into these classes. Third, it probably became clear that the majority of these techniques have not been extensively developed and evaluated. While some of the specific techniques have been evaluated thoroughly, especially the techniques described in Section 6.2.1, other techniques exist only as prototypes or experimental programs. In general, the techniques described here have shown great promise in the limited settings where they have been developed. In some cases, specific techniques have greatly accelerated skill acquisition or developed cognitive skills that were not previously being trained using conventional techniques. These techniques have yet to be integrated into a comprehensive curriculum. For the most part, each technique was developed to address a specific element of training. The design of a more complete curriculum that integrates the techniques described here has yet to be realized.

Finally, recall that the goal of these techniques is to produce a flexible agent in a decision-making or problem-solving task. Even though procedures can almost always correctly specify appropriate actions, nuclear power plant operations have produced a number of situations not entirely addressed in procedure and control room interface design. Severe accident management may be even better characterized in this way, since the procedures are likely to be less prescriptive. Developing techniques that produce operators and technical staff with better cognitive skills does not run counter to an operating philosophy that emphasizes adherence to procedures. However, when procedures exist, cognitive skills can make performance more efficient and reduce the potential for error. 


\section{References}

Anderson, J.R. (1983). The architecture of cognition. Cambridge, MA: Harvard University Press.

Aronson, E. (1978). The jigsaw classroom. Beverly Hills, CA: Sage.

Bahrick, H.P., \& Phelps, P. (1987). Retention of Spanish vocabulary over 8 years. Journal of Experimental Psychology: Learning, Memory, and Cognition, 13, 344-349.

Beatty, M.J. (1988). Increasing students' choicemaking consistency: The effect of decision rule-use training. Communication Education, 37, 95-105.

Bell, H.M. (1950). Retention of pursuit rotor task after one year. Joumal of Experimental Psychology, 40, 648-649.

Bereiter, C., \& Scardamalia, M. (1985). Cognitive coping strategies and the problem of "inert knowledge." In S. Chipman, J. Segal, \& B. Glaser (eds.), Thinking and learming skills: Research and open questions (Vol. 2). Hillsdale, NJ: Erlbaum.

Bonar, J.G. (1985). Mental models of programming loops. LRDC Technical Report. Pittsburgh, PA: Learning Research and Development Center.

Bonar, J.G., \& Cunningham, R. (1988). Bridge: Tutoring the programming process. In J. Psotka, L.D. Massey, and S.A. Mutter (eds.), Intelligent tutoring systems: Lessons learned. Hillsdale, NJ: Erlbaum.

Brown, A.L., \& Palincsar, A.S. (1989). Guided, cooperative learning and individual knowledge acquisition. In L.B. Resnick (ed.), Knowing, learning, and instruction: Essays in honor of Robert Glaser. Hillsdale, NJ: Erlbaum.

Brown, J.S., \& Burton, R.R. (1978). Diagnostic models for procedural bugs in basic mathematical skills. Cognitioe Science, 2, 155-191.

Brown, J.S., Burton, R.R., \& deKleer, J. (1982). Pedagogical, natural language and knowledge engineering techniques in SOPHIE I, II, and III. In D.
Sleeman and J.S. Brown (eds.), Intelligent tutoring systems. New York: Academic Press.

Brown, J.S., \& VanLehn, K. (1980). Repair theory: A generative theory of bugs in procedural skills. Cognitive Science, 4, 379-426.

Bukszar, E., \& Connolly, T. (1988). Hindsight bias and strategic choice: Some problems in learning from experience. Academy of Management Journal, 31, 628641.

Burton, R.R. (1982). Diagnosing bugs in a simple procedural skill. In D. Sleeman and J.S. Brown (eds.), Intelligent tutoring systems. New York: Academic Press.

Burton, R.R., \& Brown, J.S. (1979). An investigation of computer coaching for informal learning activities. International Journal of Man-Machine Studies, 11, 5-24.

Cannon-Bowers, J.A., \& Salas, E. (1990). Cognitive psychology and team training: Shared mental models in complex systems. Paper presented at the annual meeting of the Society for Industrial and Organizational Psychology, Miami, FL.

Cannon-Bowers, J.A., Salas, E., \& Converse, S.A. (1990). Cognitive psychology and team training: Shared mental models in complex systems. Human Factors Society Bulletin, 33, 1-4.

Cannon-Bowers, J.A., Salas, E., \& Converse, S.A. (1992). Shared mental models in expert team decision making. In N.J. Castellan, Jr. (ed.), Current issues in individual and group decision making, Hillsdale, NJ: Erlbaum.

Card, S., Moran, T., \& Newell, A. (1983). The psychology of human-computer interaction. Hillsdale, NJ: Erlbaum.

Carlson, R.A., Sullivan, M.A., \& Schneider, W. (1989). Component fluency in a problem-solving context. Human Factors, 31, 489-502. 
Carroll, J.B. (1976). Psychometric tests as cognitive tasks: A new "structure of intellect." In L.B. Resnick (ed.), The nature of intelligence. Hillsdale, NJ: Erlbaum.

Chase, W.G., \& Simon, H.A. (1973). Perception in chess. Cognitioe Psychology, 4, 55-81.

Chi, M.T.H., Feltovich, P.J., \& Glaser, R. (1981). Categorization and representation of physics problems by experts and novices. Cognitive Science, 5, 121-125.

Chi, M.T.H., Glaser, R., \& Farr, M.J. (1988). The nature of expertise. Hillsdale, NJ: Erlbaum.

Chi, M.T.H., \& VanLehn, K.A. (1991). The content of physics self-explanations. The Jourmal of the Learning Sciences, 1, 69-105.

Collins, A., Brown, J.S., \& Newman, S.E. (1989). Cognitive apprenticeship: Teaching the crafts of reading, writing, and mathematics. In L.B. Resnick (ed.), Knowing, learning, and instruction: Essays in honor of Robert Glaser. Hillsdale, NJ: Erlbaum.

Corbett, A.T., \& Anderson, J.R. (1992). LISP intelligent tutoring system: Research in skill acquisition. In J.H. Larkin and R.W. Chabay (eds.), Computer-assisted instruction and intelligent tutoring systems: Shared goals and complementary approaches. Hillsdale, NJ: Erlbaum.

Corker, K., Davis, L., Papazian, B., \& Pew, R. (1986). Deoelopment Of An Adoanced Task Analysis Methodology And Demonstration For Army Aircrew/Aircraft Integration. (Technical Report BBN 6124). Cambridge, MA: Bolt Beranek and Newman.

Craik, F.I.M., \& Lockhart, R.S. (1972). Levels of processing: A framework for memory research. Jourmal of Verbal Learming and Verbal Behavior, 11, 671684.

Damos, D.L., Bittner, A.C., Kennedy, R.S., \& Harbeson, M.M. (1981). Effects of extended practice on dual-task tracking performance. Human Factors, $23,627-631$.

deKleer, J. (1985). How circuits work. In D.G. Bobrow (ed.), Qualitative reasoning about physical systems. Cambridge, MA: MIT Press.
DeVries, D.L., Slavin, R.E., Fennessy, G.M., Edwards, K.J., \& Lombardo, M.M. (1980). Teams-GamesTournaments: The team learning approach. Englewood Cliffs, N]: Educational Technology Publications.

Donchin, E. (1989). The learning strategies project. Acta Psychologica, 71, 1-15.

Driskell, J.E., \& Salas, E. (1992). Can you study real teams in contrived settings?: The value of small group research to understanding teams. In R.W. Swezey and E. Salas (eds.), Teams: Their training and performance. Norwood, NJ: Ablex.

Druckman, D., \& Bjork, R.A. (1991). In the mind's eye: Enhancing human performance. Washington, DC: National Academy Press.

Druckman, D. \& Swets, J.A. (1988). Enhancing human performance: Issues, theories, and techniques.

Washington, DC: National Academy Press.

Evans, J.St.B.T. (1989). Bias in human reasoning: Causes and consequences. London: Erlbaum.

Farr, M.J. (1987). The long-term retention of knowledge and skills: A cognitive and instructional perspective. New York: Springer-Verlag.

Fendrich, D.W., Healy, A.F., Meisky, L., Crutcher, R.J., Little, W., \& Bourne, L.E. (1988). Skill maintenance: Literature review and theoretical analysis. (Technical Report AFHRL-TP-87-73). Brooks AFB, TX: Air Force Human Resources Laboratory.

Fisk, A.D., \& Lloyd, S.J. (1988). The role of stimulusto-rule consistency in learning rapid application of spatial rules. Human Factors, 30, 35-49.

Fisk, A.D., \& Schneider, W. (1983). Category and word search: Generalizing search principles to complex processing. Joumal of Experimental Psychology: Learning, Memory, and Cognition, 9, 177195.

Fitts, P.M. (1964). Perceptual-motor skill learning. In A.W. Melton (ed.), Categories of human learning. New York: Academic Press.

Fleishman, E.A. (1982). Systems for describing human tasks. American Psychologist, 37, 821-834. 
Foushee, H.C., \& Helmreich, R.L. (1988). Group interaction and flight crew performance. In E.L. Weiner \& D.C. Nagel (eds.), Human factors in aviation. San Diego, CA: Academic Press.

Franz, T.M., Prince, C., Cannon-Bowers, J.A., \& Salas, E. (1990). The identification of aircrew coordination skills. Paper presented at the 12th annual Department of Defense Symposium, Colorado Springs, CO.

Frederickson, J.R., Weaver, P.A., Warren, B.M., Gillotte, H.P., Roseberry, A.S., Freeman, B., \& Goodman, L. (1983). A componential approach to training reading skills. (Final Report 5295).

Cambridge, MA: Bolt, Beranek, and Newman.

Gentner, D., \& Gentner, D.R. (1983). Flowing waters and teeming crowds: Mental models of electricity. In D. Gentner and A.L. Stevens (eds.), Mental models. Hillsdale, NJ: Erlbaum.

Gentner, D., \& Stevens, A.L. (1983). Mental models. Hillsdale, NJ: Erlbaum.

Glaser, R. (1976). Cognitive psychology and instruction. In D. Klahr (ed.), Cognition and instruction. Hillsdale, NJ: Erlbaum.

Glenberg, A.M. (1979). Component-levels theory of the effects of spacing of repetitions on recall and recognition. Memory and Cognition, 7, 95-112.

Goldstein, I.P. (1980). Developing a computational representation for problem-solving skills. In D. Tuma and F. Reif (eds.), Problem solving and education: Issues in teaching and research. Hillsdale, NJ: Erlbaum.

Gopher, D., Weil, M., Bareket, T., \& Caspi, S. (1988). Fidelity of task structure as a guiding principle in the development of skill trainers based on complex computer games. Paper presented at the annual meeting of the Human Factors Society, Anaheim, CA.

Haber, S.B. (1989). Organization and management research support to accident management. (Task 1 Report to the Nuclear Regulatory Commission, FIN B-5879). Upton, NY: Brookhaven National Laboratory.

Hayes, J.R. (1980). Teaching problem-solving mechanisms. In D.T. Tuma and F. Reif (eds.), Problem solving and education: Issues in teaching and research. Hillsdale, NJ: Erlbaum.
Hays, R.T., \& Singer, M.J. (1989). Simulation fidelity in training system design: Bridging the gap between reality and training. New York: Springer-Verlag.

Helmreich, R.L., Chidester, T.R., Foushee, H.C., Gregorich, S., \& Wilhelm, J.A. (1989). How effective is Cockpit Resource Management Training: Issues in eoaluating the impact of programs to enhance crew coordination. (NASA/UT Tech. Rep. No. 89-2, Draft 6.0). Moffett Field, CA: NASA Ames Research Center.

Helmreich, R.L., \& Wilhelm, J.A. (1986). Evaluating CRM training: I. Measures and methodology.

(NASA/UT Tech. Rep. No. 86-8). Moffett Field, CA: NASA Ames Research Center.

Hoecker, D.G., Pople, H.E., \& Benhardt, H.C. (1991). Initiating cognitive environment simulation at Savannah River. (STC Technical Report 91-SJ4-SACES-R1 or Savannah River Lab Report WSRC-OS-91-54). Pittsburgh, PA: Westinghouse Science and Technology Center.

Hollan, J.D., Hutchins, E.L., \& Weitzman, L. (1984). STEAMER: An interactive, inspectable simulationbased training system. AI Magazine, 5, 15-27.

Johnson, D.W., \& Johnson, R.T. (1975). Learning together and alone. Englewood Cliffs, NJ: PrenticeHall.

Johnson, D.W., Maruyama, G., Johnson, R., Nelson, D., \& Skon, L. (1981). Effects of cooperative, competitive, and individualistic goal structures on achievement: A meta-analysis. Psychological Bulletin, $89,47-62$.

Kauffman, J.V., Lanik, G.F., Spence, R.A., \& Trager, E.A. (1992). Operating experience feedback report Human performance in operating events. (NUREG-1275, Vol. 8). Washington, DC: U.S. Nuclear Regulatory Commission.

Kieras, D.E., \& Bovair, S. (1984). The role of mental models in learning to operate a device. Cognitive Science, 8, 255-273.

Kieras, D.E., \& Polson, P.G. (1985). An approach to the formal analysis of user complexity. International Journal of Man-Machine Systems, 22, 365-394. 
Klein, G.A. (1989). Recognition-primed decisions. In W.R. Rouse (ed.), Adoances in man-machine systems research (Vol. 5). Greenwich, CT: JAI Press.

Klein, G., Orasanu, J., Calderwood, R., \& Zsambok, C.E. (1993). Decision making in action: Models and methods. Norwood, NJ: Ablex.

Kleinman, D.L., \& Serfaty, D. (1989). Team performance assessment in distributed decision making. In R.L. Gilson, J.P. Kincaid, \& B. Goldiez (eds.), Proceedings: Interactive networked simulation for training. Orlando, FL: Institute for Simulation and Training.

Korff, R.E. (1987). Planning as search: A quantitative approach. Artificial Intelligence, 33, 65-88.

Larkin, J.H., McDermott, J., Simon, D.P., \& Simon, H.A. (1980). Expert and novice performance in solving physics problems. Science, 208, 1335-1342.

Lauber, J.K., \& Foushee, H.C. (1981). Guidelines for line-oriented flight training. (Tech. Rep. No. NASA CP2184). Moffett Field, CA: NASA Ames Research Center.

Lave, J., Murtaugh, M., \&x de la Rocha, O. (1984). The dialectic of arithmetic in grocery shopping. In B. Rogoff \& J. Lave (eds.), Everyday cognition: Its deoelopment in social context. Cambridge, MA: Harvard University Press.

Lesgold, A., Lajoie, S., Bunzo, M., Eggan, G. (1992). SHERLOCK: A coached practice environment for an electronics troubleshooting job. In J.H. Larkin and R.W. Chabay (eds.), Computer-assisted instruction and intelligent tutoring systems: Shared goals and complementary approaches. Hillsdale, NJ: Erlbaum.

Lesgold, A., Lajoie, S., Eastman, R., Eggan, G., Gitomer, D., Glaser, R., Greenberg, L., Logan, D., Magone, M., Weiner, A., Wolf, K., \& Yengo, L. (1986). Cognitioe Task Analysis to Enhance Technical Skills Training and Assessment.. Pittsburgh, PA: Learning Research and Development Center

Lichtenstein, S., \& Fischoff, B. (1980). Training for calibration. Organizational Behavior and Human Performance, 26, 149-171.
Lintern, G., \& Roscoe, S.N. (1980). Visual cue augmentation in contact flight simulation. In S.N. Roscoe (ed.), Aviation psychology. Ames, IA: Iowa State University Press.

Loftus, E.F. (1982). Memory and its distortions. In A.G. Kraut (ed.), G. Stanley Hall lectures. Washington, DC: American Psychological Association.

Loftus, G.R. (1985). Evaluating forgetting curves. Journal of Experimental Psychology: Learning, Memory, and Cognition, 11, 397-406.

Magill, R.A., \& Hall, K.G. (1990). A review of the contextual interference effect in motor skill acquisition. Human Movement Science, 9, 241-289.

Means, B. (1983). How to choose the very best: What people know about decision-making strategies. Paper presented at the annual meeting of the American Educational Research Association, Montreal, Canada.

Means, B., \& Gott, S.P. (1988). Cognitive task analysis as a basis for tutor development: Articulating abstract knowledge representations. In J. Psotka, L.D. Massey, and S.A. Mutter (eds.), Intelligent tutoring systems: Lessons learned. Hillsdale, NJ: Erlbaum.

Means, B., Mumaw, R., Roth, C., Schlager, M., McWilliams, E., Gagne, E., Rice, V., Rosenthal, D., Heon, S. (1988). ATC training analysis study: Design of the next-generation ATC training system. (HII Technical Report). Alexandria, VA: HumRRO International, Inc.

Means, B., Salas, E., Crandall, B., \& Jacobs, T.O. (1993). Training decision makers for the real world. In G. Klein, J. Orasanu, R. Calderwood, and C.E. Zsambok (eds.), Decision making in action: Models and methods. Norwood, NJ: Ablex.

Melnick, M.J. (1971). Effects of overlearning on the retention of a gross motor skill. Research Quarterly, 42, 60-69.

Melton, A.W. (1970). The situation with respect to the spacing of repetitions and memory. Jourmal of Verbal Learning and Verbal Behavior, 9, 596-606.

Meyer, O. (1991). The on-site analysis of the human factors of operating events. (Interim Report EGGHFRU-9446). Idaho Falls, ID: INEL. 
Miller, G.A. (1956). The magical number seven, plus or minus two: Some limits of our capacity for processing information. Psychological Review, 63, 8197.

Montague, W.E. (1988). What works: Summary of research findings with implications for Navy instruction and learning. (Technical Report NAVEDTRA 115-1). Pensacola, FL: Office of the Chief of Naval Education and Training.

Montgomery, J.C., Gaddy, C.D., Lewis-Clapper, R.C., Hunt, S.T., Holmes, C.W., Spurgin, A.J., Toquam, J.L., \& Bramwell, A. (1992). Team skills eoaluation criteria for nuclear power plant control room crews. Draft manuscript.

Mumaw, R.J. (1991). Analysis and training of cognitive skills. Proceedings of the Ninth Symposium on the Training of Nuclear Facility Personnel. Oak Ridge, TN: Oak Ridge National Laboratory.

Mumaw, R.J., \& Roth, E.M. (1992). How to be more decious with a training simulator: Redefining scenarios to emphasize cognitively difficult situations. Proceedings of the annual meeting of the Simulation MultiConference: Nuclear Power Plant Simulation and Simulators, Orlando, FL.

Mumaw, R.J., Roth, E.M., \& Stubler, W.F. (1991). An analytic technique for framing control room eoaluation issues. (STC Paper 91-8SJ4-APEVL-P1). Pittsburgh, PA: Westinghouse STC.

Newell, A. (1990). Unified theories of cognition. Cambridge, MA: Harvard University Press.

Newell, A., \& Simon, H.A. (1972). Human problem solving. Englewood Cliffs, NJ: Prentice-Hall.

Orasanu, J. (1990). Shared mental models and crew decision making. Paper presented at the annual meeting of the Cognitive Science Society, Cambridge, MA.

Oser, R., McCallum, G.A., Salas, E., \& Morgan, B.B. (1989). Toward a definition of teamwork: An analysis of critical team behaviors. (Technical Report 89-004). Orlando, FL: Naval Training Systems Center.
Palincsar, A.S., \& Brown, A.L. (1984). Reciprocal teaching of comprehension-fostering and monitoring activities. Cognition and Instruction, 1, 117-175.

Papert, S. (1980). Mindstorms: Children, computers, and powerful ideas. New, York: Basic Books.

Payne, J.W., Bettman, J.R., \& Johnson, E.J. (1988). Adaptive strategy selection in decision making. Journal of Experimental Psychology: Learning, Memory, and Cognition, 14, 534-552.

Perrow, C. (1984). Normal accidents. New York: Basic Books.

Pew, R.W., Miller, D.C., \& Feeher, C.E. (1981). Evaluations of proposed control room improvements through analysis of critical operator decisions. (NP-1982, Research Project 891). Cambridge, MA: Bolt, Beranek, \& Newman, Inc.

Pirolli, P.L., \& Greeno, J.G. (1988). The problem space of instructional design. In J. Psotka, L.D. Massey, and S.A. Mutter (eds.), Intelligent tutoring systems: Lessons learned. Hillsdale, NJ: Erlbaum.

Prince, C., Chidester, T.R., Bowers, C., \& CannonBowers, J. (1992). Aircrew coordination: Achieving teamwork in the cockpit. In R.W. Swezey and E. Salas (eds.), Teams: Their training and performance. Norwood, NJ: Ablex.

Rasmussen, J. (1980). The human as a systems component. In H.T. Smith and T.R.G. Green (eds.), Human interaction with computers. London: Academic Press.

Rasmussen, J. (1986). Information processing and human-machine interaction: An approach to cognitive engineering. New York: North-Holland.

Reason, J. (1990). Human error. Cambridge: Cambridge University Press.

Reder, L. (1979). The role of elaborations in memory for prose. Cognitive Psychology, 11, 221-234.

Reiser, B.J. (1987). Causal models in programming. Paper presented at the third international conference on artificial intelligence and education, Pittsburgh, PA. 
Reiser, B.J., Kimberg, D.Y., Lovett, M.C., \& Ranney, $M$. (1992). Knowledge representation and explanation in GIL: An intelligent tutor for programming. In J.H. Larkin and R.W. Chabay (eds.) Computer-assisted instruction and intelligent tutoring systems: Shared goals and complementary approaches. Hillsdale, NJ: Erlbaum.

Roth, E.M., \& Mumaw, R.J. (1994). Cognitive engineering. In J.J. Marciniak (ed.), Encyclopedia of software engineering. New York: Wiley-Interscience.

Roth, E.M., \& Woods, D.D. (1988). Aiding human performance: I. Cognitive analysis. Le Travail Humain, $51,39-64$.

Roth, E.M., Woods, D.D., \& Pople, H.E. (1992) Cognitive simulation as a tool for cognitive task analysis. Ergonomics, 35, 1163-1198.

Rouse, W.B., \& Morris, N.M. (1986). On looking into the black box: Prospects and limits in the search for mental models. Psychological Bulletin, 100, 349-363.

Salas, E., Dickinson, T.L., Converse, S.A., \& Tannenbaum, S.I. (1992). Toward an understanding of team performance and training. In R.W. Swezey and E. Salas (eds.), Teams: Their training and performance. Norwood, NJ: Ablex.

Scardamalia, M., \& Bereiter, C. (1985). Fostering the development of self-regulation in children's knowledge processing. In S.F. Chipman, J.W. Segal and R. Glaser (eds.), Thinking and learning skills: Research and open questions. Hillsdale, NJ: Erlbaum.

Schendel, J.D., \& Hagman, J.D. (1982). On sustaining procedural skills over a prolonged retention interval. Journal of Applied Psychology, 67, 605-610.

Schneider, W. (1985). Training high-performance skills: Fallacies and guidelines. Human Factors, 27 285-300.

Schneider, W., \& Detweiler, M. (1988). The role of practice in dual-task performance: Toward workload modeling in a connectionist/control architecture. Human Factors, 30, 539-566.

Schneider, W., \& Fisk, A.D. (1982). Concurrent automatic and controlled visual search: Can processing occur without resource cost? Journal of

\section{Experimental Psychology: Learning, Memory, and Cognition, 8, 261-278.}

Schneider, W. \& Shiffrin, R.M. (1977). Controlled and automatic human information processing: $I$.

Detection, search, and attention. Psychological Review, 84, 1-66.

Schoenfeld, A.H. (1985). Mathematical problem solving. New York: Academic Press.

Sharan, S. (1980). Cooperative learning in small groups: Recent methods and effects on achievement, attitudes, and ethnic relations. Review of Educational Research, 50, 241-271.

Sharan, S., \& Sharan, Y. (1976). Small-group teaching. Englewood Cliffs, NJ: Educational Technology Publications.

Simon, H.A. (1957). Models of man. New York: Wiley.

Slamecka, M.J., \& Graf, P. (1978). The generation effect: Delineation of a phenomenon. Journal of Experimental Psychology: Human Learning and Memory, $4,592-604$.

Slavin, R.E. (1983). Cooperative learning. New York: Longman.

Slavin, R.E. (1988). Principles of effective instruction. Washington, DC: National Academy Press.

Smith, S.M., \& Rothkopf, E.Z. (1984). Contextual enrichment and distribution of practice in the classroom. Cognition and Instruction, 1, 341-358.

Sticha, P.J., Schlager, M., Buede, D., Epstein, K., \& Blacksten, H.R. (1990). Specifying skill-based training strategies and devices: A model description. (Final Report FR-PRD-90-01). Alexandria, VA: HumRRO.

Sturges, P., Ellis, J., \& Wulfeck, W. (1981). Effects of performance-oriented text upon long-term retention of factual material. (NPRDC Technical Report 81-22). San Diego, CA: Navy Personnel Research and Development Center.

Tolcott, M.A., Marvin, F.F., \& Bresnick, T.A. (1989). The confirmation bias in military situation assessment. Reston, VA: Decision Science Consortium. 
Tversky, A., \& Kahneman, D. (1974). Judgment under uncertainty: Heuristics and biases. Science, 185, 112431.

U.S. Nuclear Regulatory Commission. (1988). Individual plant examination. Generic Letter 88-20. Washington, DC.

U.S. Nuclear Regulatory Commission. (1989). Staff plans for accident management regulatory and research programs. SECY-89-012. Washington, DC.

U.S. Nuclear Regulatory Commission. (1989). Severe accident risks: An assessment for five U.S. nuclear power plants. NUREG-1150. Washington, DC.

U.S. Nuclear Regulatory Commission (1989).

Individual plant examinations: Submittal guidance. NUREG-1335. Washington, DC.

U.S. Nuclear Regulatory Commission (1993). Operator licensing examiner standards. NUREG-1021 - Revision 7. Washington, DC.

VanLehn, K. (1982). Bugs are not enough: Empirical studies of bugs, impasses, and repairs in procedural skills. The Journal of Mathematical Behavior, 3, 3-71.

VanLehn, K. (1988). Toward a theory of impassedriven learning. In $\mathrm{H}$. Mandl and A. Lesgold (eds.), Learning issues for intelligent tutoring systems. New York: Springer-Verlag.

Vidulich, M., Yeh, Y., \& Schneider, W. (1983). Timecompressed components for air intercept control skills. In Proceedings of the Human Factors Society 27th Annual Meeting. Santa Monica, CA: Human Factors Society.

Wenger, E. (1987). Artificial intelligence and tutoring systems: Computational and cognitive approaches to the communication of knowledge. Los Altos, CA: Morgan Kaufmann.

White, B.Y., \& Frederiksen, J.R. (1986). Progressions of qualitative models as a foundation for intelligent learning environments. (Report No. 6277). Cambridge, MA:

Bolt, Beranek, and Newman.

Woods, D.D. (1982). Operator decision behavior during the Steam Generator Tube Rupture at the Ginna nuclear power station. (Report 82-1C57-CONRM-R2). Pittsburgh, PA: Westinghouse Electric Corp.

Woods, D.D, \& Hollnagel, E. (1987). Mapping cognitive demands in complex problem-solving worlds. International Journal of Man-Machine Studies, $26,257-275$.

Zakay, D., \& Wooler, S. (1984). Time pressure, training, and decision effectiveness. Ergonomics, 27, 273-284. 


\title{
Appendix A
}

\section{Scenario Development Methodology}

\author{
Table of Contents
}

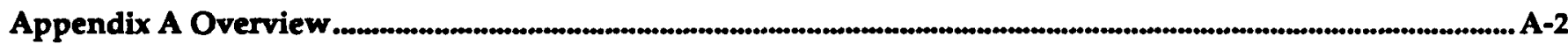

A.1 Scenario Development ....n.

A.1.1 Assumptions about the Eventual Scope and Depth of SAM Guidance ....................................................A-2

A.1.2 Attributes Considered in Selecting HLAs for Scenario Design .......................................................................3

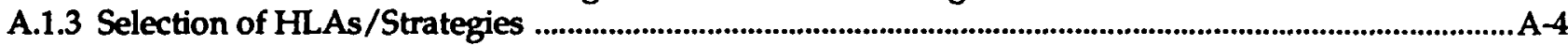

A.2 Scenario Use

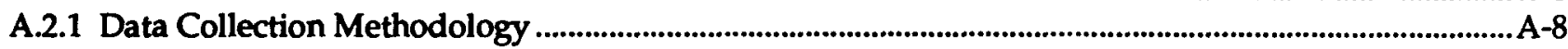

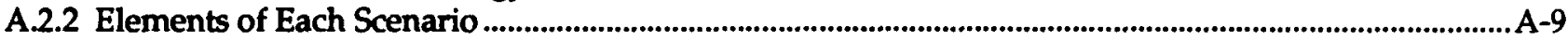

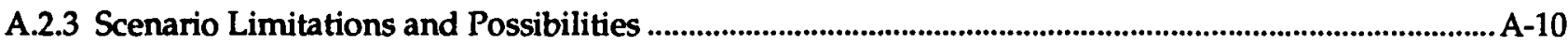

Tables

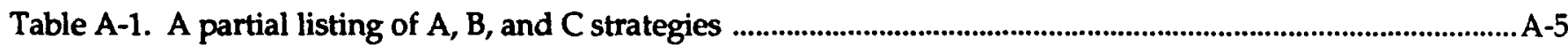




\section{Appendix A Overview}

The 12 severe accident management scenarios developed for this project can be found in Appendices $B$ and C. Appendix A provides an account of the methodology used to develop these scenarios and a description of the structure and use of the scenarios. Section A.1, which describes scenario development, focuses on the process used to identify and select among high-level actions and accident management strategies. The second section describes the elements of each scenario and the process developed for presenting the scenarios to panels of utility personnel.

It is important to note that the PWR and BWR scenarios developed for this project are not intended to represent the specific severe accident events that are most likely to occur; they are not based on statistical analyses of expected failure frequencies. These scenarios are only intended to present possible severe accident scenarios in order to place panels of utility SAM staff (composed of control room, TSC, and EOF personnel) into novel and difficult decisionmaking situations that might occur during a severe accident.

\section{A.1 Scenario Development}

Development of SAM scenarios was a multi-step process that started with a basic assumption about the eventual scope and depth of SAM guidance, including assumptions about the subset of high-level actions (HLAs) that would eventually be included in the owners' group guidance documents. A set of attributes or characteristics that scenarios should have was then identified. Recall that one of the goals of scenario development was to introduce complexities that would push decision-making teams outside normal procedures for making decisions. A set of HLAs was then selected from those being discussed in the mid-1991 industry literature as candidates for inclusion in SAM guidance. Finally, scenarios were designed based upon the specific attributes of the selected HLAs.

\section{A.1.1 Assumptions About the Eventual Scope and Depth of SAM Guidance}

To aid scenario development, the following assumptions were made about the eventual scope and depth of SAM guidance:

- Some, but not all, of the candidate high-level actions (HLAs) under general consideration in mid-1991 would be retained in eventual SAM guidance.

- Eventually, SAM guidelines (SAMGs) would be developed, along with a definition of their relationship to existing emergency operating procedures (EOPs).

- There could be cases in which it would be difficult to determine whether a given SAM guideline should be applied during accident management.

- The TSC and other groups are likely to be involved in technical decisions that go beyond the EOPs.

One early decision was the identification of candidate HLAs. The PWR scenarios were developed during the late summer of 1991; the BWR scenarios during the early fall of 1991 . These development efforts predated the availability of owners' group SAM guidance. However, a great deal of other SAM technical literature was available at that time. For example, the following publications, among others, were consulted:

- NUREG/CR-5682 - Specific Topics in Severe Accident Management

- NUREG/CR-5474 - Assessment of Candidate Accident Management Strategies

- NUREG/CR-5447 - Depressurization as an Accident Management Strategy to Minimize the Consequences of Direct Containment Heating

- NUREG/CR-5263 - The Risk Management Implications of NUREG-1150 Methods and Results

- NUREG/CR-4177 - Management of Severe Accidents

- NUREG-1150 - Severe Accident Risks: An Assessment for Five U.S. Nuclear Power Plants

- SECY-89-012 - Staff Plans for Accident Management Regulatory and Research Programs 
- Proceedings of a Workshop on Severe Accident Management for PWRs, May 15-17, 1990, University of California, Los Angeles

- Proceedings of a Workshop on Severe Accident Management for BWRs, September 26-28, 1990, University of California, Los Angeles

In much of this literature, especially in NRC reports and in NRC contractor reports, HLAs that could potentially prevent or mitigate a severe accident were referred to as candidate SAM "strategies." Many of these "strategies" were later called high-level actions (HLAs) in the generic guidance developed by each of the owners' groups. The distinction between an HLA and a strategy is based on hierarchy and level of detail. An HLA is a generic action that can be taken to mitigate a severe accident. For example, "inject into the RPV/RCS (reactor pressure vessel/ reactor coolant system)" is an HLA. A strategy specifies the plant system/ equipment to be used in performing an HLA; it is a specific method for implementing an HLA. For example, "inject into the RPV/RCS using charging pumps" is a strategy.

By the middle of 1991, literally dozens of HLAs/ strategies were being discussed in the literature. Owners' group SAM guidance was anticipated but not yet available. It seemed clear that some subset (but not all) of the candidate HLAs/ strategies then under discussion in the literature would be retained in the generic SAM guidance developed by each of the owners' groups. After obtaining expert opinion, certain HLAs were eliminated from consideration and others were given greater consideration.

These assumptions had the following impact on scenario development. Scenario design was NOT driven by a particular, predetermined sequence of events. Rather, scenario design was directed at reaching a desired set of plant conditions for which a proposed HLA/ strategy would be (or appear to be) appropriate. Thus, because of these early assumptions, the task of selection turned out to be not among scenarios but among candidate HLAs/ strategies for which scenarios could then be designed to provide pretext and context. The exact sequence of events specified in a scenario is important mainly in that it leads to the desired set of severe accident conditions and that it provides a frame of reference from which the panel can begin to evaluate the HLA/ strategy that is being posed as an option for consideration.

\section{A.1.2 Attributes Considered in Selecting HLAs for Scenario Design}

In addition to assumptions about the eventual form and content of SAM guidance, a set of attributes, or complicating factors, to consider when selecting candidate HLAs/ strategies as focal points for scenario design was identified. To increase complexity in decision making in a non-artificial manner, the candidate HLA/strategy should have a high likelihood of being retained in eventual SAM guidance and should be described in one or more of the following ways:

- HLA is NOT in the current EOPs

- HLA conflicts with guidance in the current EOPs

- HLA is suggested as a possible action by the current EOPs, but the EOPs provide little or no guidance for evaluating or implementing the action

- HLA challenges common misconceptions or ingrained notions

- HLA challenges the limits of the current knowledge and understanding of severe accident phenomenology

It was not necessary that each HLA selected exhibit all of the identified attributes. The attributes were identified to help select a mix of scenarios suitable for the project's data collection needs.

As a first criterion, it was critical that a candidate HLA/ strategy have a high likelihood of being retained through the EPRI Technical Basis Report (TBR) development process for incorporation into owners' group SAM guidance. Estimation of the likelihood for retention was based on the pros and cons argued for various HLAs in the literature available in mid-1991. The goal in this regard was that a majority (but NOT all) of the HLAs/ strategies selected for scenario development be consistent with the HLAs that eventually emerged in SAM guidance. For a few scenarios, HLAs/ strategies were proposed that were either based on current misconceptions about severe accidents or that, for some other reason, were considered as unlikely or marginal candidates for eventual inclusion within SAM guidance. The following are examples of HLAs for which scenarios were developed and that, as of January 1993, were under consideration for inclusion within owners' group SAM guidance: 


\section{HLA:}

Inject into RPV/RCS Operate Fan Coolers Inject into Containment Depressurize RPV/RCS Vent containment Spray into containment

\section{Scenario Number:}

PWR-1 and BWR-4 PWR-2

PWR-3 and BWR-3

PWR-4

BWR-2

BWR-6

Aside from the likelihood of retention in eventual SAM guidance, the HLA attributes identified were all factors that in some way complicated the decisionmaking process. The aim in identifying these attributes was to specify how and in what ways the decision making required within the context of the scenarios would be more difficult than standard decision making. The following examples reflect development of PWR scenarios; BWR scenarios provide similar examples.

Some of the HLA attributes contributing to difficult decision making were that the HLA/strategy NOT be in the current EOPs or that it conflict with guidance in the current EOPs. For example, the HLA "Inject into containment" did not exist within the PWR generic network of emergency response guidelines (ERGs). The HLA of "Stopping all containment fan coolers" in scenario PWR-2 conflicted with the generic Westinghouse owners' group (WOG) ERG guidance (ECA-0.2).

Another potentially complicating factor was that the HLA/ strategy was suggested as a possible action by the current EOPs, but the EOPs provided little or no guidance for evaluating or implementing the action. For example, at the end of the generic WOG ERG ECA-1.1 the operators are directed to check whether the RCS pressure and temperature requirements are met for operation of the RHR system in the shutdown cooling mode. Even if the requirements are met, the operators are directed to consult the TSC in order to determine if the RHR system should actually be placed in service in the shutdown cooling mode. However, no guidance or evaluation criteria are provided for the TSC.

Accordingly, scenario PWR-5 was developed to include a proposed recommendation in which it is suggested that the ECCS be maintained in the injection mode, even though the plant is approaching the condition for which the EOPs direct the operators to transfer the ECCS to the recirculation mode.

Additional attributes were that the HLA/ strategy challenge common misconceptions or ingrained notions; or that it challenge the limits of the current knowledge and understanding of severe accident phenomenology. For example, in scenario PWR-1 the recommendation to inject unborated water into the RPV may appear to be unacceptable to some SAM personnel because it challenges the ERG-based notion that subcriticality is the highest-priority critical safety function (CSF).

\section{A.1.3 Selection of HLAs/Strategies}

By mid-1991, when the scenarios were created, a categorization scheme had come into common usage in which many of the candidate HLAs/ strategies then under general consideration were divided into three categories: "A," "B," and " $\mathrm{C}$ " strategies. Generally, the " $A$ " strategies were preventive in nature; the " $B$ " strategies, mitigative. The " $C$ " strategies were a set of additional mitigative strategies for BWRs. The "A" and " $B$ " categories included some strategies applicable only to PWRs or BWRs and some applicable to both.

The "A" strategies were published in NUREG/CR5474, "Assessment of Candidate Accident Management Strategies." Generally, these strategies help prevent severe core damage from occurring in the first place, provided that they can be implemented. NUREG/CR-5474 contains a comprehensive list of " $A$ " strategies for both PWRs and BWRs.

Generally, the " $\mathrm{B}$ " and " $\mathrm{C}$ " strategies help mitigate the consequences and /or progression of severe core damage that has already occurred. The "B" strategies for PWRs were published in the May 1990 UCLA SAM Workshop Proceedings (and later published in NUREG/CR-5781). The "B" strategies for BWRs and the "C" strategies (additional BWR mitigative strategies) were published in the September 1990 UCLA SAM Workshop Proceedings (and later published in NUREG/CR-5780). The " $A$ ", "B", and " $C$ " strategy categories captured most of the HLAs/ strategies appearing in the literature in mid-1991.

Table A-1 presents a partial listing of these categories. This Table also shows which strategies were incorporated into the 12 scenarios. The strategies are listed under more general functional categories. Note 
Table A-1. A partial listing of A, B, and C strategies. Unless otherwise noted, the strategies are applicable to both PWRs and BWRs. Those put forth solely or primarily for PWRs are followed by (PWR); those for BWRs, by (BWR). The strategies that we selected for scenario development are underlined. The underlined strategies are followed by an indication of the scenario in which the strategy was used.

\section{"A" (Preventive Strategies)}

Category 1: Maintain coolant inventory

- Refill the RWST with borated water (PWR)

- Reduce containment spray flow rate to conserve water for core injection (PWR-6)

- Use charging pumps for core injection (PWR-1)

- Use alternate injection for RCP seal cooling (PWR)

- Refill the CST with condensate (BWR)

- Use CRD pumps for core injection (BWR)

- Extend ECCS availability by switching pump suction from suppression pool (BWR)

- Raise or bypass RCIC trip set-point to extend availability (BWR)

Category 2: Maintain decay heat removal

- Use condensate or start-up pumps for injection into steam generator (PWR)

- Enable emergency cross-tie of service water and CCW to feedwater (PWR)

- Use diesel-driven fire pump for injection into steam generator (PWR-5)

- Use diesel-driven fire pump for injection to containment sprays

- Re-open MSIVs and turbine bypass valves to regain main condenser as heat sink

- Enable emergency connection of feedwater to river, reservoirs, or municipal water systems

- Use diesel-driven fire pump for core injection (BWR-4)

- Enable emergency cross-tie of service water to RHR (BWR)

Category 3: Control reactivity

- Ensure an abundant supply of borated water

- Initiate SLCS (BWR-1)

Category 4: Maintain support systems

- Conserve battery capacity by shedding non-essential loads

- Use portable battery charger to recharge batteries

- Enable emergency cross-tie of AC power between two units or to on-site gas turbine generator

- Enable emergency replenishment of gas supply, or otherwise ensure the operability of air-operated components

- Use diesel generator or gas-turbine generator to power CRD pumps for core injection (BWR) 
Table A-1 (continued)

$$
\text { "B" and "C" (Mitigative Strategies) }
$$

Category 1: Prevent vessel failure

- Use RCS pumps to force flow through the core (PWR)

- Depressurize and inject coolant into the RCS (PWR)

- Remove RCS heat using steam generators (secondary feed and bleed) (PWR-5)

- Remove RCS heat using PORVs (primary bleed and feed) (PWR)

- Flood the reactor cavity for external cooling of the reactor vessel (PWR-3)

- Use CRD pumps for vessel injection (BWR)

- Use fire pump for vessel injection (BWR-4)

- Use fire pumps for drywell flooding up to the bottom head of the reactor vessel (BWR)

Category 2: Prevent containment failure due to slow over-pressurization

- Use containment sprays to remove containment heat (PWR)

- Use fan coolers to remove containment heat (PWR-2)

- Flood cavity before or after vessel failure to delay / prevent core-concrete interaction (PWR-3)

- Use recombiners or ignitors to control combustible gases (PWR-2)

- Vent containment to relieve pressure

- Recover RHR (BWR)

- Use fire pump for containment sprays (BWR)

Category 3: Prevent containment failure due to rapid over-pressurization

- Depressurize RCS to prevent direct containment heating (PWR-4)

- Flood cavity before or after vessel failure to break up and cool core debris (PWR-3)

- Vent containment to control combustible gases (PWR-2)

- Vent containment during ATWT (BWR-2)

- Control hydrogen (BWR)

Category 4: Prevent failure due to missiles

- Dry vessel to eliminate in-vessel steam explosions (PWR)

- Maintain RCS at high pressure to prevent steam explosions (PWR)

Category 5: Mitigate fission product release

- Use auxiliary pressurizer spray to scrub fission products before release through PORV (PWR)

- Depressurize RCS for steam generator tube rupture (PWR)

- Flood steam generator secondary for tube rupture (PWR)

- Flood location of containment leakage

- Depressurize containment to reduce driving force for leakage

- Flood break location for interfacing system LOCA 
that some scenarios incorporate more than one strategy. Also, note that the scenario BWR-5 (see page 4 of Appendix C) does not incorporate any of these strategies, but instead focuses on other aspects of decision making.

\section{A.2. Scenario Use}

\section{A.2.1 Data Collection Methodology}

Data were to be collected by conducting a series of table-top exercises in which severe accident scenarios would be presented to panels of utility personnel who are likely to have SAM responsibilities. Presentation of each scenario was to be followed by questioning of the panel regarding a proposed "recommendation" offered, as part of the scenario, by a fictitious member of the control room or TSC staff. The proposed recommendation is offered as a means of mitigating the severe accident condition.

The proposed recommendation was deliberately chosen to present the panel with a problem for which little specific procedural guidance exists. The recommendation was also chosen to test/ confirm the elements of the decision-making model. That is, an attempt would be made to determine whether the actual process that the panel goes through is consistent with the model. The panel was expected to evaluate the merits of the recommendation and the means of implementing it.

The objective of the questioning was to determine how the panel would perform the task, and to observe the kinds of obstacles and difficulties impeding the decision-making process and the kinds of cognitive skills that the panel uses to overcome these impediments. Questions related to the proposed recommendation were designed to do the following:

- Get the panel members to articulate the decision-making process as they move through it, identifying sources of information, point of authority, number of outside inputs, etc.

- Keep the panel focused on the central issues related to the proposed recommendation.

- Verify the performance model and associated cognitive skills that are required to support the decision-making process.
Although the structure of SAM organizations varies among utilities, the panels would generally be composed of the following types of utility personnel: operations (SRO, shift supervisor, STA), TSC and EOF (engineering and technical support personnel), emergency planning, PRA types familiar with the facility IPE. Panel size was targeted between four and six members, based on the desire to strike a balance between the following objectives: obtain a large sample of the utility's expertise, promote good group dynamics, and minimize impact on the utility's resources.

The scenarios were to be presented by a data collection team consisting of a cognitive psychologist, a PWR or BWR technical specialist (who developed the scenarios), and a technical expert from the utility. The data collection team was to meet in advance of the panel session to review the scenarios with the utility technical expert. The specific details contained within the scenarios are based on generic reference plants-specifically, SNUPPS for the PWR scenarios and the BWR-4 reactor with a Mark I containment for the BWR scenarios (refer to section A.2.3.) The intent of the advance meeting was to modify the scenario to make it more consistent with the following plantspecific aspects: systems and equipment configuration, structure of the emergency organization, emergency plan and implementation procedures.

The panel sessions were to be run as informal, tabletop, group exercises. The panel would be told the purpose of the exercises. These exercises were NOT intended to be used to assess the panel's competence, neither as a group nor as individuals, or to evaluate the facility's procedures or emergency organization. Questions are asked to determine the elements of the decision-making process and to identify the cognitive skills needed to support that process. There are no "right" or "wrong" answers as such.

The technical members of the investigating team were then to present the following scenario sections to the panel: Statement for Panel, Recommendation, and Background Material for Conducting Exercise. See Appendices $B$ and $C$ for the actual scenarios.

The Statement for Panel accounts for how plant conditions deteriorated to their current severeaccident state. The Recommendation is offered as a proposed mitigative action of uncertain or unknown 
Table A-1 (continued)

$$
\text { "C" strategies: }
$$

- Reflood reactor vessel with borated water (BWR-1)

- Flood the entire containment (BWR)

- Flood the top head of the Mark II dry-well (BWR)

\section{Other Strategies}

From the "Proceedings of a Workshop on Severe Accident Management for BWRs"

- Inject neutron poison (BWR)

- Initiate dry-well spray to mitigate HPME (BWR-6)

- Flood dry-well containment following large LOCA (BWR-3)

- Flood dry-well containment during non-LOCA event to prevent vessel failure (BWR) 
efficacy; it may or may not prove to be a good idea. It may be provocative in that it conflicts with existing guidance or runs contrary to ingrained notions. The Background Material for Conducting Exercise defines the degraded plant state in terms of equipment status and instrumentation indications. This scenario section provides information needed to assess CSFs and to evaluate the Recommendation and associated implementation strategy.

As each of these scenario sections was presented, the panel members were to be given an opportunity to seek clarification from the technical members of the investigating team. The questioning of the panel could then proceed as follows. An initial question gets the panel focused on evaluating the Recommendation. The panel has at its disposal the technical references that would be available during an actual accident. The panel is to work as a team and try to arrive at a consensus.

The initial, follow-up, and probing questions were to be posed by the technical members of the investigating team, who were also to provide additional information to the panel upon request. Responses to such requests were to be based upon the information contained within the Background Material and to be consistent with the assumptions underlying the Statement. A document listing all of these assumptions was to be available to the investigating team but NOT to the panel. The cognitive psychologist was to observe the panel's decision-making process and to interact with the panel members as necessary to obtain clarification about that process.

\section{A.2.2 Elements of Each Scenario}

Each scenario was developed to include the following sections:

Scenario Objective. This section identifies the basic objectives of the scenario in terms of the central issue(s) with which the panel will be faced. This section is not intended for presentation to the panel. It is a high-level statement of the problem posed by the scenario, provided for the investigators and for purposes of documentation.

Scenario Assumptions. This section lists the technical assumptions upon which the scenario is based. This section is not intended for presentation to the panel.
However, the assumptions in this section do inform the narrative in the Statement for Panel section, which is presented to the panel. Accordingly, the assumptions postulate a set of initial plant conditions and a sequence of events (including the timing of those events) that trace the evolution of the accident to its current state.

Additionally, the assumptions are intended to provide a frame of reference from which the technical members of the investigating team can respond to panel requests for additional information.

Accordingly, this section contains information that anticipates the panel members' needs for additional information, both in terms of the questions they are likely to ask and in terms of the decisions they might make.

Statement for Panel. This section is a narrative account of how plant conditions deteriorated to their current severe-accident status. It IS intended for presentation to the panel members. It orients them to the accident history and to the current plant conditions. It is an essential part of the context within which the Recommendation will be proposed.

This section includes time-line information from the Assumptions section regarding the sequence and timing of major events and operator actions, presented from the perspective of the control room. It also tracks the procedural usage of the operating crew, including the declaration of emergency action levels. This helps orient the panel to the ERG network and to the emergency plan implementation procedure (EPIP); plant conditions may be at the fringe of or beyond the ERG network.

The Recommendation, which concludes this section, proposes an HLA that is offered as a means of mitigating the severe accident condition. This statement includes a brief summary of the concerns and line of reasoning that motivate the proposal. A strategy for implementing the HLA may also be suggested (or it may be left to the panel to devise one). The Recommendation attempts to focus the panel's attention on a central problem related to managing the accident. It delineates the pertinent issues that must be discussed and resolved.

It is intended that the panel members view the Recommendation as a suggestion (put forth by a fictitious member of the control room or TSC staff) 
that should be subjected to their evaluation. The fact that the Recommendation is made is not meant to imply anything regarding its merits, one way or the other. It may be controversial or provocative in that it conflicts with existing guidance or runs contrary to ingrained notions. It includes the opinions of the Recommendation proponent, his/her concerns, thinking-out-loud, and speculation about the future course of the accident.

Initial Questions to Panel and Follow-up or Probing Questions. This section contains questions related to the proposed Recommendation that are designed to: start the panel members working on the problem and keep them moving along; get the panel members to articulate as they go through the decision-making process; keep the panel focused on the central issues related to the proposed Recommendation; ascertain the panel's assessment of the Recommendation; probe the decision-making process; verify the cognitive skills that are required to support the decisionmaking process.

Background Material for Conducting Exercise. This section, which includes a description of current plant conditions, defines the degraded plant state in terms of equipment status and instrumentation indications. This section provides information needed to assess CSFs and to evaluate the feasibility and potential effectiveness of the Recommendation and associated implementation strategy.

Connections to Decision-Making Model. This section provides a connection between the cognitive skills identified in the performance model and the specifics of the scenario. For example, the performance model may indicate that a certain type of knowledge is required to determine implications in terms of plant control goals or critical safety functions. This section identifies scenario-specific knowledge that is connected to the more general skill description. These connections lead to further questions that can be asked of the panel.

\section{A.2.3 Scenario Limitations and Possibilities}

The scenarios in Appendices B and C were developed for a specific purpose that limits their use in other settings. However, there are potential uses in addition to the limitations.
Limitations can be grouped into four categories: their extremely low probability, historical context, their table-top nature, their use of a generic reference plant.

Events have Extremely Low Probability. The selection of scenarios for use in this project was not based upon considerations regarding probability of occurrence. It must be recognized that severe accidents, exceeding the design basis of the plant and involving a degraded core, are by nature very lowprobability events. For a given plant, the probability that a severe accident will happen at all is quite low. Lower still is the probability of occurrence for a specific severe accident involving a particular sequence of events. Consequently, the scenarios that must be postulated to reach severe accident conditions are inherently unlikely.

A combination of equipment failure and human error, such as happened at TMI-2, would probably be the most likely cause of a severe accident. However, the accident sequences used in these scenarios emphasize failed equipment almost to the point of excluding human error, which may further reduce the likelihood of these scenarios. The decision to base the scenarios almost entirely on equipment failure derived from previous experience that showed that it was generally counterproductive to drive training scenarios or "questionnaire-type" scenarios on the basis of human error.

Events were Developed in Unusual Historical Context. The scenarios were developed in mid-1991, before the availability of owners' group SAM guidance. Consequently, the development efforts were not informed by the final content of that guidance and the associated documentation (e.g., EPRI TBR). However, some foreknowledge of the eventual owners' group SAM guidance could be gleaned from general trends in the evolution of severe accident management that had already become apparent by the middle of 1991.

For example, it was clear that for some owners' groups the TSC would have the primary decisionmaking authority in technical decisions that go beyond the EOPs. Additionally, the basic group of mitigative HLAs and some of their associated implementing strategies were also known from the general literature, including NRC publications. This foreknowledge regarding the eventual SAM guidance is reflected in the scenarios developed for this project. 
Table-Top Nature of the Scenarios. The scenarios developed for this project were designed solely as table-top exercises. It was never intended or contemplated that they be used in conjunction with a plant-specific simulator or with any other kind of simulation.

The event sequences and timelines assumed in the scenarios were incorporated only to provide the background and context that the panel would need to assess plant conditions, evaluate the

Recommendation, and devise an implementation strategy. It was intended that the Statement for Panel and Background Material sections be provided to the panel members in writing, with an opportunity for them to obtain clarification via questioning of the technical members of the investigating team. Similarly, it was intended that the panel members address any additional requests for information to the investigating team.

Events Use a Generic Reference Plant. For a reference plant, the PWR scenarios assume a generic SNUPPS plant, which is a four-loop, 3411-MWt NSSS housed in a large, dry containment. For an emergency procedure network, the PWR scenarios assume Revision 1A of the generic ERGs maintained by the Westinghouse Owners' Group.

The BWR scenarios were initially drafted to be used for a generic BWR-4 with a Mark I containment. Although the BWR scenarios were generalized somewhat after their initial draft, they are still more applicable to Mark I containment types. For an emergency procedure network, the BWR scenarios assume the generic EPGs maintained by the BWR Owners' Group.

There are strong limitations on the ways in which the 12 scenarios should be used. However, these scenarios may be useful as table-top exercises for utility personnel becoming familiar with some of the potential difficulties of SAM. Eventually, SAM guidance documents will have to be tested at each utility. These scenarios, within the constraints mentioned above, can serve as a starting point for difficult test scenarios. Furthermore, the scenarios reveal a qualitatively different type of complexity that can be introduced into a scenario. 


\title{
Appendix B
}

\section{PWR Scenarios}

\author{
Table of Contents
}

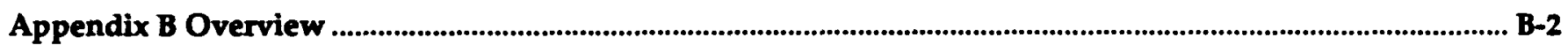

B.1 Scenario Summaries ................................................................................................................................................ B-2

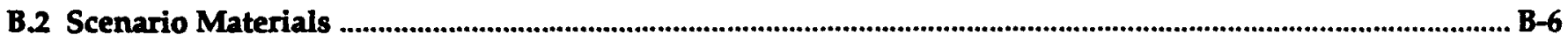

PWR-1: Injection with Unborated Water.................................................................................................................6-6

PWR-2: Containment Cooling versus Inert Atmosphere ..................................................................................... B-12

PWR-3: Deliberate Flooding of Reactor Cavity ......................................................................................................17

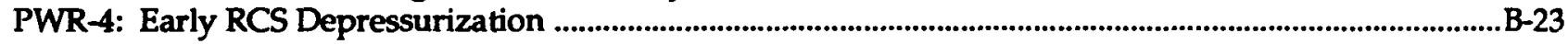

PWR-5: Natural Circulation versus RHR Cooling ............................................................................................

PWR-6: Containment Spray versus ECCS Injection ............................................................................................ B-34

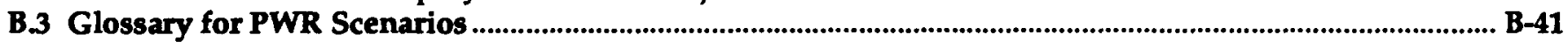




\section{Appendix B Overview}

This Appendix is split into two sections. The first section, Scenario Summaries, provides capsulized descriptions of each of the PWR scenarios. The second section, Scenario Materials, contains the detailed descriptions of each scenario and provides the detailed support material required to present each scenario to a panel (as described in Appendix A). A glossary of scenario acronyms can be found at the end of the Appendix.

\section{B.1 Scenario Summaries}

This section provides brief descriptions of the 6 PWR scenarios that were created for this project.

PWR-1: Injection with Unborated Water. The purpose of this scenario is to eliminate the borated injection water sources and force the panel members to decide whether to inject unborated water.

In this scenario, an earthquake results in the following situation. The RWST and the boric acid storage tanks are damaged. The inventory of borated water stored in these tanks becomes depleted and cannot be replaced. Although sufficient water accumulates in the containment sump to allow core cooling to be transferred to the recirculation mode, the transfer cannot be effected. The HLA contained within the recommendation proposed in this scenario is to "Inject into the RPV/RCS." We anticipate that this HLA will be retained in owners' group SAM guidance.

The recommendation proposed in this scenario contains a strategy for implementing the HLA. The strategy is to line up an unborated water source (the reactor make-up water storage tank, which is not part of the ECCS) to the suction of the charging pumps and inject into the core.

Some SAM personnel may be inclined to reject the strategy because of a concern about re-criticality (apparent conflict with guidance in current EOPs). The proposed strategy might appear to conflict with the established priority of EOP critical safety functions (CSFs) in which subcriticality has the highest ranking. Additionally, a tendency to reject the proposed strategy might also stem from a failure to recognize that criticality is acceptable, provided that sufficient heat (fission, decay, zirc-water) can be removed to keep the core cool (challenge a common misconception). A perceived goal conflict between subcriticality and core cooling could make the decision to accept or reject the proposed recommendation very difficult for some SAM personnel (challenge an ingrained notion).

\section{PWR-2: Containment Cooling versus Inert}

Atmosphere. The purpose of this scenario is to force the panel members to evaluate and resolve a goal conflict between continued containment cooling and maintenance of an inert containment atmosphere.

In this scenario, an earthquake causes a LOCA and an extended loss of all AC power. A period of severe core damage (clad oxidation and melt) ensues, during which hydrogen in the containment atmosphere reaches a concentration at which it would be burnable if it were mixed with dry air. However, the containment atmosphere is effectively inerted by the large amount of steam that escapes from the RCS during the blowdown phase of the LOCA. An AC emergency bus is eventually energized and safeguards loads, including two containment fan cooler units, are started. The HLA contained within the recommendation proposed in this scenario is to "Operate (Stop) Fan Coolers." We anticipate that this HLA will be retained in owners' group SAM guidance.

The recommendation proposed in this scenario to stop the containment fan coolers appears to conflict with the procedural guidance of ECA-0.2, which explicitly directs the operators to start the fan coolers in the emergency mode. The recommendation also challenges the ingrained notion that reducing containment pressure and temperature via spray and/or fan coolers is always consistent with preserving/ restoring the containment CSF.

The scenario also presents technical problems and difficult decisions related to management of the containment hydrogen concentration with and without the availability of a hydrogen recombiner. With no recombiner available, the panel must decide whether to use the hydro-gen purge system. This involves venting the containment, following a period of clad melting (and possible eutectic dissolution of some fuel), in order to control combustible gases. 
PWR-3: Deliberate Flooding of Reactor Cavity. This scenario creates a situation in which the core is known to be severely damaged already and in which failure of the reactor vessel might be imminent. The purpose is to force the panel members to decide whether or not to flood the reactor cavity deliberately as a means of mitigating the consequences of a potential reactor vessel failure.

This scenario initiates from a shutdown condition with the RCS drained to the mid-loop elevation and with nozzle dams installed in the hot and cold legs of all steam generators (SGs). The secondary sides of all SGs are in wet lay-up. Because of miscommunications, the size of the RCS hot-leg-side vent is inadequate and a primary manway on the cold-leg side of one of the SGs is open. An earthquake causes loss of all $A C$ power and damages the controls for one of the PZR PORVs. As the coolant in the RPV heats up and RPV pressure increases, $a$ cold-leg nozzle dam fails, allowing much of the RPV coolant inventory to be ejected via the open SG manway. Only limited gravity feed to the RPV can be established from the RWST. Some of the fuel melts and relocates into a non-coolable geometry. After several hours, an AC emergency bus is re-energized and high-head hot-leg injection is established in accordance with abnormal response guideline ARG-1.

The HLA contained within the recommendation proposed in this scenario is to "Spray into containment." However, the goal stated in the recommendation is to flood the reactor cavity with water, in anticipation of potential RPV failure. The recommendation asserts that the cavity water will quench the pour of molten corium and, thereby, protect the containment basemat. This goal is normally associated with the HLA to "Inject into containment." We anticipate that both HLAs, "Spray into containment" and "Inject into containment" will be retained in owners' group SAM guidance.

Although current EOPs contained the HLA "Spray into containment," the purpose of containment spray, within the context of the ERG network, was NOT to flood the reactor cavity. The recommendation proposed in this scenario gives flooding of the reactor cavity as the main purpose for spraying the containment in this case. Current generic EOPs did not contain the HLA "Inject into containment."
The suggestion contained within the proposed recommendation that external cooling might prevent RPV failure challenged the current limits of knowledge and understanding about severe-accident phenomenology.

PWR-4: Early RCS Depressurization. The purposes of this scenario are to create a situation in which the potential for fuel melting and RPV failure is extremely high and to force the panel members to decide whether to depressurize the RCS early in the accident deliberately as a means of preventing or mitigating direct containment heating (DCH).

In this scenario, an earthquake causes loss of all $\mathrm{AC}$ power and loss of all auxiliary (emergency) feedwater. This results in a loss-of-feed-induced LOCA. Given the estimated repair time required before regaining either an emergency bus or a secondary heat sink, fuel melting appears to be a virtual certainty. The HLA contained within the recommendation proposed in this scenario is to "Depressurize the RPV/RCS." We anticipate that this HLA will be retained in owners' group SAM guidance.

The recommendation proposed in this scenario contains a strategy for HLA implementation. The strategy is to use the PZR PORVs and to do so as soon as possible, even before inade-quate core cooling (ICC) symptoms, as defined in the ERGs, arise ("early" depressurization). This proposal conflicts with the ERG-based notion that the PZR PORVs should not be latched open unless an RCS feed path has already been established or except as a last resort after the onset of ICC and failure of other preferred HLAs to reduce core-exit temperatures.

The recommendation proposed in this scenario also raises the specter of high-pressure melt ejection (HPME) and of direct containment heating (DCH). These concerns, especially the DCH concern, challenged the current limits of knowledge and understanding about severe-accident phenomenology.

The recommendation proposed in this scenario involves an implicit goal conflict between core cooling and containment. Containment would be challenged by $\mathrm{DCH}$, which can be precluded by preventing HPME, which in turn can be prevented by depressurizing the RPV/RCS. Thus, in this case, the 
ultimate purpose of depressurizing the RCS is to preserve containment. However, early depressurization of the RCS accelerates the depletion rate of RCS inventory and hastens the onset of core heatup. Hence, there is a conflict between addressing the potential threat to containment and maintaining core cooling.

\section{PWR-5: Natural Circulation versus RHR Cooling.}

The purposes of this scenario are to create a situation in which the core has overheated and for which the guidance provided in the existing emergency procedures may be perceived as counter-productive. The intent is to force the panel members to decide whether to transfer to cold-leg recirculation on RHR or to try to establish RCS natural-circulation cooling.

In this scenario, an earthquake causes loss of all AC power and loss of all normal and emergency feedwater. This results in a loss-of-feed-induced LOCA. Only one AC emergency bus can be recovered, off-site power remains unavailable. Safety injection flow is established and RCS bleed-and-feed cooling is initiated. Extensive cladding failure has occurred.

In this scenario, hazards perceived as being associated with transfer to cold-leg recirculation are presented. A proposal is made to restore the secondary heat sink and establish natural circulation instead of transferring to cold-leg recirculation. The panel members must evaluate the perceived hazards of recirculation, assess the technical feasibility of restoring the secondary heat sink, and estimate the likelihood of establishing RCS natural circulation. The recommendation proposed in this scenario contains two HLAs: "Inject into SGs" and "Depressurize SGs." We anticipate that these HLAs will be retained in owners' group SAM guidance.

The recommendation proposed in this scenario conflicts with the guidance provided by the ERG critical safety function restoration procedure in effect (FR-H.1, Loss of Secondary Heat Sink). The ERG directs the operators to transfer the ECCS to the recirculation mode when RWST level decreases to a predetermined level. At this time, the safety injection system is feeding the RCS from the RWST during RCS bleed-and-feed heat removal.

The recommendation proposes leaving the ECCS in the injection mode while trying to re-establish the secondary heat sink. The reason given for the proposed procedural deviation is a concern that ECCS recirculation will cause high radiation levels outside of containment and increase the potential for off-site releases via containment bypass.

A similar concern is reflected in some ERGs in which the operators are directed to consult the TSC staff before placing the RHR system in service in the shutdown-cooling mode (for example: ES-1.2, PostLOCA Cooldown and Depressurization; and ECA-1.1, Loss of Emergency Coolant Recirculation). Although the concern about radiation levels and potential releases is part of the background for directing the operators to consult the TSC, the TSC is not typically provided with any guidance or criteria for evaluating the concern.

Generic ERG steps involving transfer to ECCS recirculation contain no such directions for the operators to consult the TSC. Thus, the recommendation raises the radiation and release concern in a novel context and under a set of circumstances that make it difficult to simply dismiss the concern.

PWR-6: Containment Spray versus ECCS Iniection. The purposes of this scenario are to create a situation in which the containment fan cooler units (CFCUs) and emergency coolant recirculation (ECR) capability have been lost and in which RWST inventory is being rapidly depleted by containment spray. The intent is to force the panel members to decide whether to sacrifice containment cooling by terminating sprays in order to preserve ECCS injection capability.

In this scenario, an earthquake causes a LOCA, loss of all $A C$ power, and loss of emergency coolant recirculation capability. After extensive cladding oxidation has occurred, two $\mathrm{AC}$ emergency buses are re-energized. Later, a hydrogen burn occurs in containment, actuating containment spray. All containment fan cooler units are lost. ECR remains unavailable while containment spray is rapidly depleting RWST inventory. The HLA contained within the recommendation proposed in this scenario is to "Inject into the RPV/RCS." We anticipate that this HLA will be retained in owners' group SAM guidance.

The recommendation proposed in this scenario contains a strategy for implementing the HLA. The 
strategy is aimed at conserving RWST inventory for injection into the RPV/RCS by stopping the single running containment spray pump, which is drawing down RWST inventory to supply spray. The desire to conserve RWST inventory stems from the fact that emergency coolant recirculation capability has been lost.

The proposal to stop all containment spray under the existing plant conditions conflicts with the ERG procedure in effect (ECA-1.1, Loss of Emergency Coolant Recirculation). The ERG directs the operators to leave one containment spray pump running when containment pressure is at the value cited in the scenario and when no containment fan cooler is running.

The recommendation proposed in this scenario involves an implicit goal conflict between core cooling and containment. In this case, the containment CSF, as defined in the ERGs, is not satisfied. Consequently, the ERG requires some form of active containment cooling.

The core cooling CSF can potentially be first severely, and then extremely, challenged if the RWST becomes totally depleted and recirculation remains unavailable. At that point, all injection into the RPV/RCS would be lost. Continuation of containment spray accelerates the depletion of RWST inventory. Thus, there is a conflict between maintaining core cooling and maintaining containment cooling. 


\section{B.2 Scenario Materials}

PWR-1: Injection with Unborated Water

\section{Warning:}

This scenario is intended to be used as a tool or exercise for generating a response from a small accidentmanagement team (or panel) to a hypothetical severe accident. The function of this accident scenario is to present a situation that pushes the team outside of the standard, well-rehearsed procedures for addressing abnormal plant conditions. To achieve this, we created scenarios with difficult choices, goal conflicts, or unique situations that present significant challenges to the team's decision-making process.

Further, to create the necessary severe accident conditions in this scenario, it was necessary to postulate numerous traumas, equipment failures, and at times, operator errors. The use of multiple failures was required to thwart the safety and defense-in-depth design employed in nuclear power plants and reduce the effectiveness of current emergency operating procedures (EOPs).

The boundary conditions of the scenario were constructed for the sole purpose of maintaining a difficult decisionmaking situation for as long as possible. Therefore, it should not be construed that these scenarios are likely events, or even the most likely severe accident sequences. The probability of these scenarios occurring is within, or beyond, the range of traditional IPE core-damage frequencies.

\section{Scenario Objective:}

The objectives of this scenario are as follows:

- To place the panel members in an imminent core-melt situation for which the existing emergency procedures are ineffective.

- To eliminate the borated injection water sources and force the panel members to decide whether or not to inject unborated water via a line-up that includes non-safety-grade piping and components.

- To present technical problems to the injection of unborated water that force panel members to devise a solution that is not available in current procedures.

\section{Scenario Assumptions:}

These assumptions are for the presenter and are not to be presented to the panel:

- SNUPPS plant; early in fuel cycle; critical boron concentration at hot full power is $900 \mathrm{ppm}$.

- Earthquake of magnitude greater than the SSE causes four-inch cold-leg break with coincident loss of off-site power and damage to safeguards systems.

- Manual reactor trip and automatic safety injection.

- RWST suffers extensive structural damage; its contents spill; the combination of spillage and ECCS usage accelerates the depletion of RWST inventory.

- Each boric acid transfer-pump suction line is severed from its respective boric-acid storage tank; tanks quickly drain.

- Accumulators inject 12 minutes $(720 \mathrm{sec}$ ) after trip and SI.

- Aftershock of magnitude approximating that of OBE occurs 55 minutes after the initial shock and severs the ECCS suction line and the make-up line from RWST.

- Power available to RHR pumps and containment recirc sump isolation valves. 
- Transition from E-1 to ES-1.2 occurs while RCS pressure is still above the shut-off head of the RHR pumps and before RWST level drops to the criterion for switchover to cold-leg recirculation.

- Upon reaching step 5 of ES-1.2, the operators leave the RHR pumps running.

- Upon reaching step 3a of ES-1.3, the operators discover that the recirc-sump-to-RHR-suction isolation valves (EJ HIS-8811A and EJ HIS-8811B) will not open, even though electrical power is available.

\section{Statement for Panel:}

The plant had been operating at $100 \%$ power for 45 days. The critical boron concentration at hot full power was $900 \mathrm{ppm}$. About two hours ago, an earthquake of magnitude greater than the SSE occurred. The control room operators manually tripped the reactor. Safety injection actuated automatically. The earthquake caused a loss of off-site power and some damage to safeguards systems and components.

The RWST suffered extensive structural damage; its contents spilled uncontrollably through various breaches in the tank. The combination of spillage and ECCS usage accelerated the depletion of RWST inventory.

Additionally, each boric-acid transfer-pump suction line was severed from its respective boric-acid storage tank. The boric-acid storage tanks rapidly drained.

The control room operators entered E- 0 and declared a Site Emergency. The TSC and the EOF were activated. Upon reaching the diagnostic steps of E-0, the operators transitioned to E-1 and initiated monitoring of CSF status trees. Upon determining that RCS cooldown and depressurization were required, the operators transitioned to ES-1.2. Thirty minutes after the trip and SI, with RCS pressure at 398 psia and RCS hot leg temperature at 444 F (RCS saturation conditions), the operators commenced dumping steam via the steam generator PORVs.

The control room operators recognized the rapid depletion of RWST inventory. However, because of the loss of off-site power and the damage to the boric-acid storage system, they were unable to make-up to the RWST.

As RWST level indication dropped below the set-point for automatic switch-over to cold-leg recirculation, the operators realized that switch-over did not automatically occur. Forty-two minutes after SI, with RCS pressure at 329 psia and RCS hot leg temperature at $426 \mathrm{~F}$ (RCS saturation conditions), the operators determined that there was sufficient inventory in the containment recirculation sump to allow transfer to cold-leg recirculation ${ }^{1}$.

While attempting to implement ES-1.3, the operators discovered that the recirc-sump-to-RHR-suction isolation valves (EJ HIS-8811A and EJ HIS-8811B) would not open, even though electrical power was available ${ }^{2}$. The emergency classification level was upgraded to that of General Emergency.

Fifty minutes after the trip and SI, RWST level indication was approaching 5\%. The operators placed the control handswitches for all pumps taking a suction on the RWST in the pull-to-lock position. Fifty-five minutes after the

\footnotetext{
1 Assume SI mass flow rate of $50 \mathrm{lbm} / \mathrm{sec}$ for $2520 \mathrm{sec}(42 \mathrm{~min}$.) and $8 \mathrm{lbm} / \mathrm{gal}: 15,750 \mathrm{gal}$ in containment sump. Some panel members may suggest that the operators should have placed RHR in service in the shutdown cooling mode at this point. If this issue comes up, handle it in the following way: The operators did recognize that RCS pressure was low enough to allow shutdown cooling (for SNUPPS, RCS pressure must be less than 395 psia to allow shutdown cooling). They also recognized that RCS hot-leg temperature was 76 degrees above the maximum allowable for going on shutdown cooling. Furthermore, a significant LOCA is in progress. The operators realize that if they simply place shutdown cooling in service, then RCS inventory will eventually be depleted to the point that the RHR pumps lose suction on the RCS hot legs. Even now, the RCS is at saturation; placing shutdown cooling in service may lead to cavitation or vapor binding of the RHR pumps.

2 If the question comes up, the recirc-sump-to-containment-spray-suction isolation valves (EN HIS-1 and EN HIS-7) will not open either, even though electrical power is available.
} 
trip and SI, an aftershock of magnitude approximating that of the OBE occurred. The ECCS suction line and the make-up line were severed from the RWST, which emptied completely.

The operators transitioned to ECA-1.1 and depressurized all steam generators to atmospheric. Ninety minutes after the trip and SI, the control room operators and TSC personnel agreed that the RHR system should not be placed in service in the shutdown cooling mode. Their decision was based on the following plant conditions: reactor vessel water level, as indicated by the RVLIS static range, was $50 \%$ and decreasing; RCS hot-leg temperature was $448 \mathrm{~F}$ and increasing; $\mathrm{RCS}$ pressure was 414 psia.

\section{Recommendation:}

One hour and fifty-five minutes after the reactor trip and SI, a control room operator made the following recommendation:

"If we don't get water into the core soon, it will overheat. I think we should try to line up the reactor makeup water storage tank to the suction of the charging pumps and cool the core that way."

\section{Initial Questions to Panel:}

- Should this recommendation be implemented? Why or why not?

- How might you modify the recommendation?

- If it were adopted, how would you actually go about planning for and implementing the recommendation?

\section{Follow-up or Probing Questions:}

- Do the panel members perceive a goal conflict between cooling the core by injecting unborated water and maintaining subcriticality?

- NUREG ICR-5574, Assessment of Candidate Accident Management Strategies, foresees the potential that accident management personnel might perceive a conflict between cooling the core and maintaining subcriticality: "...this strategy advocates the use of RWST refill sources having sufficient boron concentration to maintain an appropriate reactor safe reactivity shutdown margin. The use of unborated refill water can potentially raise concern over recriticality if its use results in sufficiently diluted water being injected into the reactor. This is especially true at the beginning of a fuel cycle when the required operating boron concentrations are relatively high."

- Do the panel members recognize that the plant is in a condition for which the existing EOPs contain no specific actions that will be effective in preventing/mitigating core overheat?

- The scenario has the operators in ECA-1.1 at the time the recommendation is made to inject unborated water. All of the actions of ECA-1.1, including depressurization of the steam generators to atmospheric and consideration of the possibility of placing shutdown cooling in service, have already been completed. Some plant-specific versions of ECA-1.1 may address, to some level of detail, the issue of making up to the RCS from sources other than the RWST, but it is unlikely that any existing procedural guidance was ever intended to cover a case in which the reactor make-up system is so extensively disabled.

- Some panel members may believe that they are essentially "stuck" in ECA-1.1 or ES-1.3 because of the note at the beginning of ES-1.3 which states that function restoration procedures (FRPs) should not be implemented until the recirc-sump-to-RHR-suction flow path has been established. Even if the operators transition to FRC.2 or FR-C.1, the actions contained therein either have already been completed or will have no significant impact on core overheat.

- If the panel members devise a means to get water to the suction of the charging pumps, do they give any consideration to the probable need for throttling the discharge flow rate from the charging pump(s)? 
- Any improvised water source will probably only be able supply a relatively low flow rate to the suction of the charging pump(s). If the discharge flow rate is not throttled, the charging pump(s) will likely cavitate and may be damaged.

- One way to address this problem is to run only a single charging pump. Additionally, the PDP has a lower volumetric flow capacity than a CCP and could be run instead of a CCP because it has a lower suction flow demand.

- The safety injection charging lineup through the BIT is a low-resistance flow path and is much less amenable to throttling than is the normal charging flow path. So, the panel might plan to isolate the BIT and establish normal charging.

\section{Background Material for Conducting Exercise:}

For SNUPPS, the line-up proposed by the Recommendation presents the following technical challenges:

1. The reactor make-up water transfer pumps are powered from the service buses, which are de-energized because of the loss of off-site power. Although it would be possible to establish a valve line-up from the reactor make-up water storage tank to the suction of the charging pumps, it is doubtful that gravity flow from the MWST through the idle make-up water transfer pumps would be sufficient to sustain charging pump operation, even if the charging pump discharge flow rate were severely throttled. Charging pump suction flow and suction pressure would still be inadequate.

2. Aside from the lack of power for the reactor make-up water transfer pumps, the valve lineup from the MWST to the suction of the charging pumps could be established as follows:

- Close the RWST-to-charging-pump-suction isolation valves (LCV-112D via handswitch BN HIS-112D; and

LCV-112E via handswitch BN HIS-112E). If these valves are inoperable or inaccessible because of damage in the vicinity of the RWST, then check valves $8546 \mathrm{~A}$ and $8546 \mathrm{~B}$ will prevent backflow to the RWST.

Additionally, there is a manual isolation valve in the line from the RWST (V011) that the operators might be able to close.

- Open the VCT-to-charging-pump-suction isolation valves (LCV-112B via handswitch BN HIS-112B; and LCV-112C via handswitch BN HIS-112C). This is necessary so that water from the blending tee and/or the

VCT can reach the suction of the charging pumps.

- Line up the reactor make-up system for dilution or alternate dilution, as per the normal operating procedure.

If the line-up cannot be established by using the make-up-mode selector switch, then the individual control handswitches on the main control board can be used to establish the line-up. Failing that, local manual operation of the valves could be attempted. In addition to the normal dilution flow paths, manual valve V183 can be opened to establish a flow path from the discharge of the reactor make-up water transfer pumps directly to the suction of the charging pumps.

The following considers the implications of modifying the scenario by leaving the boric-acid storage system intact:

For SNUPPS, a recommendation to transfer water from the boric-acid storage tanks to the suction of the charging pumps via the boric-acid transfer pumps would have been a more viable recommendation than the one made by the control room operator, given the loss of power to the reactor make-up water transfer pumps.

The boric-acid transfer pumps are powered from the AC emergency buses and therefore would be available, according to the scenario. Whether the boric-acid transfer pumps could supply sufficient flow and pressure to the suction of the charging pumps to allow sustained operation of the charging pumps is 
doubtful. However, if the charging pump discharge flow rate were throttled, the boric-acid transfer pumps would be able to "keep up" with the suction demands of the charging pumps.

An effective way of throttling the charging pump discharge flow rate is to isolate the flow path through the BIT and establish normal charging. This was done on the SNUPPS II simulator and the boric-acid transfer pumps were able to sustain a charging flow rate of $150 \mathrm{gpm}$. To achieve this it was necessary to open the "immediate boration" flow path (i.e., to open HV-8104). In fact, with HV-8104 open and both boric-acid transfer pumps running, it was possible to secure the normal boration flow path and isolate the charging pump suction from the VCT as well as from the RWST.

\section{Current plant conditions, two hours after the trip and SI, are as follows:}

- RCS hot-leg temperature: $\mathbf{4 8 0} \mathrm{F}$, increasing

- RCS pressure: 566 psia, increasing

- RVLIS static range: $35 \%$, decreasing

- Core exit thermocouple temperature: $500 \mathrm{~F}$, increasing

- AC emergency buses: both energized

- AC service buses: all deenergized

- Emergency classification level: General Emergency

- Handswitches in pull-to-lock

- Charging pumps

- SI pumps

- RHR pumps

- Containment spray pumps

- RWST is empty; the make-up line and the ECCS suction line have been severed from the RWST; emergency repairs will take several days to effect

- Both boric-acid storage tanks are empty; each boric-acid transfer-pump suction line has been severed from its respective boric-acid storage tank

- LOCA and loss of the following: off-site power, boric-acid storage tanks, boric-acid transfer pumps, RWST and make-up capability, safety injection flow, emergency recirculation capability

\section{Plant-Specific Information:}

Boric acid transfer pumps (2)

Capacity, each: 75 gpm at $235 \mathrm{ft}$

Power supply: vital 480 vac (NG01A and NG02A)

Boric acid storage tanks (2)

Capacity, each: 24,000 gal

Concentration: $4 \mathrm{w} / \mathrm{o}$ (7000 ppm)

Boric acid batching tank (1)

Capacity: $800 \mathrm{gal}$

Boron injection makeup pump (1)

Capacity: $35 \mathrm{gpm}$ at $250 \mathrm{ft}$

Reactor makeup water storage tank (1)

Capacity: 150,000 gal

Concentration: unborated water

Reactor makeup water transfer pumps (2) 
Capacity, each: $150 \mathrm{gpm}$ at $300 \mathrm{ft}$

Power supply: non-vital 480 vac (PG19N, PG20N)

Procedure Titles:

E-0, Reactor Trip or Safety Injection

E-1, Loss of Reactor or Secondary Coolant

ES-1.2, Post LOCA Cooldown and Depressurization

ES-1.3, Transfer to Cold Leg Recirculation

ECA-1.1, Loss of Emergency Coolant Recirculation

FR-C.1, Response to Inadequate Core Cooling

FR-C.2, Response to Degraded Core Cooling

Connections to Decision-Making Model:

Interpret Current State:

- Knowledge of plant-specific conditions, faults present, systems lost/unavailable: In reading the Statement, do the panel members grasp that

- All SI and ECCS recirc capability has been lost?

- Make-up to RWST is lost?

- BAST and BATPs lost?

Determine Implications:

- Knowledge of functional systems and higher-level processes: Does it register on the panel members that ALL borated water sources are unavailable?

- Knowledge of criteria for violating critical safety functions: Do the panel members realize that

- Core cooling is already in a "degraded" state?

- The core exit is super-heating?

- ICC will occur as soon as core-exit temperature exceeds $700 \mathrm{~F}$ ?

Plan:

- Knowledge of limits of applicability for EOPs: Do the panel members recognize that the plant is in a condition for which the existing EOPs contain no specific actions that will be effective in preventing/mitigating core overheat?

- Knowledge of criteria for applying HLA (conditions of applicability): Do the panel members perceive a goal conflict between core cooling and subcriticality in the proposed HLA to add unborated water? Do they determine that a return to criticality is acceptable if core cooling is re-established and sustained?

Control:

- Knowledge of specific plant systems required for implementing plan or procedure: Can the panel members devise or improvise a method of injecting unborated (or borated) water into the RCS that is effective and feasible? 
PWR-2: Containment Cooling versus Inert Atmosphere

\section{Warning:}

This scenario is intended to be used as a tool or exercise for generating a response from a small accidentmanagement team (or panel) to a hypothetical severe accident. The function of this accident scenario is to present a situation that pushes the team outside of the standard, well-rehearsed procedures for addressing abnormal plant conditions. To achieve this, we created scenarios with difficult choices, goal conflicts, or unique situations that present significant challenges to the team's decision-making process.

Further, to create the necessary severe accident conditions in this scenario, it was necessary to postulate numerous traumas, equipment failures, and at times, operator errors. The use of multiple failures was required to thwart the safety and defense-in-depth design employed in nuclear power plants and reduce the effectiveness of current emergency operating procedures (EOPs).

The boundary conditions of the scenario were constructed for the sole purpose of maintaining a difficult decisionmaking situation for as long as possible. Therefore, it should not be construed that these scenarios are likely events, or even the most likely severe accident sequences. The probability of these scenarios occurring is within, or beyond, the range of traditional IPE core-damage frequencies.

\section{Scenario Objective:}

The objectives of this scenario are as follows:

- To place the panel members in a situation in which there has already been severe core damage (clad oxidation and melt) during which hydrogen in the containment atmosphere reached a concentration at which it would be burnable if it were mixed with dry air. However, during the same period of severe core damage, the containment atmosphere was effectively inerted by the large amount of steam that escaped from the RCS.

- To restore containment fan cooler operation and containment spray capability and force the panel members to decide whether to continue containment cooling.

- To present technical problems to the management of the containment hydrogen concentration that force the panel members to devise a solution that is not available in current procedures. (If it is also postulated that the $\mathrm{H} 2$ recombiners are unusable, the panel will have to decide whether to use the $\mathrm{H} 2$ purge system.)

\section{Scenario Assumptions:}

These assumptions are for the presenter and are not to be presented to the panel:

- SNUPPS plant; early in fuel cycle; critical boron concentration at hot full power is $900 \mathrm{ppm}$

- Earthquake of magnitude greater than the SSE causes 4-inch cold-leg break with coincident loss of all AC power and damage to safeguards systems

- Manual reactor trip and automatic safety injection actuation signal

- Accumulators inject 12 minutes $(720 \mathrm{sec}$ ) into the event

- RCP seals deteriorate and develop leakage to the containment; the seal leakage rate reaches 50 gpm per pump and then decreases as RCS pressure decreases

- Core uncovery begins at 65 minutes into the event

- Core melt begins at 94 minutes into the event

- Core slump and collapse begin at 108 minutes into the event 
- The 4-kV safeguards bus associated with emergency diesel generator (EDG) " $A$ " is so badly damaged that it cannot be energized, even if EDG " $A$ " were to be started or if off-site power were to be restored; nor can it be energized even if EDG "B" were available to be cross-connected

- Some of the train " $\mathrm{B}$ " 480-V safeguards electrical distribution equipment is damaged, including the power cable to the "B" hydrogen recombiner

- EDG " $B$ " is started and connected to its associated 4-kV safeguards bus at 120 minutes into the event

- The " $\mathrm{B}$ " train of safeguards equipment is manually loaded onto the reenergized bus, as follows:

- Essential service water pump (1)

- Component cooling water pump (1)

- RHR pump (1)

- SI pump (1)

- Containment fan cooler units (2)

- Hydrogen mixing fan (2)

- Instrument air compressor (1)

- Centrifugal charging pump (1)

- The "B" containment spray pump is placed in stand-by

- The "B" motor-driven AFW pump is left in "pull-to-lock"

\section{Statement for Panel:}

The plant had been operating at $100 \%$ power for 45 days. The critical boron concentration at hot full power was 900 ppm. About two hours ago, an earthquake of magnitude greater than the SSE occurred. The control room operators manually tripped the reactor. Safety injection actuated automatically. The earthquake caused a loss of all $\mathrm{AC}$ power and some damage to safeguards systems and components.

The control room operators entered ECA- 0.0 and declared a Site Emergency. The TSC and the EOF were activated. From RCS and containment indications, the operators determined that the RCS was not intact. The early onset of significant primary LOCA symptoms led the operators to conclude that the RCS pressure boundary had already been breached prior to and independent of the development of any RCP seal leakage.

Twenty minutes into the event, the emergency classification level was upgraded to that of General Emergency. In accordance with ECA- 0.0 , the operators depressurized the steam generators to 230 psig via manual operation of the PORVs. They verified containment isolation and containment ventilation isolation.

Plant operations, maintenance, and technical support personnel and off-site personnel engaged in coordinated efforts to start the emergency diesel generators (EDGs), to restore off-site power, and to conserve DC power supplies. However, because of the extensive repairs required, all $A C$ buses remained de-energized for two hours. After determining that 4-kV safeguards bus " $\mathrm{A}$ " was too extensively damaged to be energized even if a power source became available, plant personnel concentrated their efforts on starting emergency diesel generator (EDG) "B".

During the time that the plant was without $\mathrm{AC}$ power, conditions in the RCS, core, and containment deteriorated. Core uncovery began at 65 minutes into the event, as indicated on the static range of RVLIS. At 75 minutes into the event, RCS pressure was 250 psig and RCS hot-leg temperature reached $700 \mathrm{~F}$. At 105 minutes into the event, core-exit thermocouple temperatures reached $2300 \mathrm{~F}$.

At 120 minutes into the event, the following indications existed:

- RCS pressure: 200 psig and slowly decreasing

- RCS hot-leg temperature: $700 \mathrm{~F}$

- RVLIS static range: $10 \%$

- Core-exit thermocouple temperature (avg): $3500 \mathrm{~F}$ 
- Containment pressure: 10 psig and slowly increasing

- Containment temperature: $170 \mathrm{~F}$ and slowly increasing

- Containment humidity: $100 \%$

- Containment radiation:

- Air-particulate activity detector

- Gaseous activity detector

- Containment (high-range) area radiation monitor(s)

- Containment hydrogen concentration: $8.5 \%$ in dry air, increasing

Two hours into the event, EDG "B" was started and connected to 4-kV safeguards bus "B". The control room operators transitioned to ECA-0.2. They manually loaded the following "B"-train safeguards equipment onto the re-energized bus:

- Essential service water pump (1)

- Component cooling water pump (1)

- RHR pump (1)

- SI pump (1)

- Containment fan cooler units (2)

- Hydrogen mixing fan (2)

- Instrument air compressor (1)

- Centrifugal charging pump (1)

\section{Recommendation:}

Two hours and fifteen minutes into the event, after learning that the control room operators had started two containment fan cooler units, an engineer in the TSC makes the following recommendation:

"I think we should stop the containment fan coolers until we get the hydrogen concentration reduced. If we condense the steam that is currently in the containment atmosphere, we might be left with a detonable or burnable mixture of hydrogen and air."

\section{Initial Questions to Panel:}

- Should this recommendation be implemented? Why or why not?

- How would you evaluate the apparent conflict between the engineer's recommendation and the procedural guidance of ECA-0.2, which explicitly directs the operators to start the fan coolers in the emergency mode? Could you reconcile the conflict?

- Assume that it was decided to stop the containment fan cooler units while a containment hydrogen sample was obtained and analyzed.

- How high could the resulting hydrogen concentration be while still allowing for immediate restart of the fan coolers?

- If the hydrogen concentration were found to be above your highest acceptable level for fan cooler operation, how would you reduce the hydrogen concentration below that level?

- How would you proceed if hydrogen recombiner "B" failed to operate? (Assume the power cable had been severed at the containment penetration.)

- Does your plant have an alternate means of reducing the containment hydrogen concentration?

- If you have a hydrogen purge system, would you use it?

\section{Background Material for Conducting Exercise:}

Current Plant Conditions, 15 minutes after restoration of power to safeguards bus " $\mathrm{B}$ ", are as follows: 
- RCS pressure: 200 psig and slowly decreasing

- RCS hot-leg temperature: $700 \mathrm{~F}$

- RVLIS static range: $25 \%$ and slowly increasing

- Core-exit thermocouple temperature (avg): $1100 \mathrm{~F}$ and decreasing at ? F/min

- Containment pressure: 10 psig and slowly decreasing

- Containment temperature: $170 \mathrm{~F}$ and slowly decreasing

- Containment humidity: $98 \%$

- Containment radiation:

- Air particulate activity detector

- Gaseous activity detector

- Containment (high-range) area radiation monitor(s)

- Containment hydrogen concentration: $9 \%$ in dry air

- AC emergency buses: 4-kV bus "B" energized

- AC service buses: all de-energized

- Emergency classification level: General Emergency

- Equipment in "pull-to-lock"

- Motor-driven AFW pump "B"

- All safeguards train "A" equipment

- Assumptions: LOCA and loss of all AC power; core melt; hydrogen concentration burnable in dry air; steaminerted containment atmosphere; loss of both hydrogen recombiners; T-hot RTD pegged high; CETs track to 3500F

\section{Connections to Decision-Making Model:}

\section{Monitor /Detect:}

- Ability to differentiate between instrument failure and accident response: Do the panel members "believe" the indications of severe core overheat-interpret the readings as indications of severe core overheat?

\section{Interpret Current State:}

- Ability to develop complete and accurate mental representation of plant status: Do the panel members try to assess the following:

- Has core cooling been restored by flow from the operating train of safety injection?

- Has the progression of core damage been arrested?

- With one complete train of safety injection restored, can or should anything else be done to address the core cooling CSF?

Do the panel members realize that containment integrity should now be the highest priority because

- Fission products were previously released to the containment?

- Containment hydrogen concentration is burnable in dry air?

\section{Determine Implications:}

- Ability to anticipate phenomenological consequences of action/inaction: Do the panel members realize that passive cooling (by structural heat sinks and heat losses to ambient) will tend to de-inert the containment atmosphere by condensing steam, even if all active cooling (coolers and spray) is stopped?

Plan:

- Knowledge of criteria for applying HLAs (conditions of applicability)

- Critical parameter values 
- Systems that must be available

- Knowledge of specific plant systems required for implementing plan or procedure: Do the panel members devise a plan that includes the following elements?

- Operation of hydrogen recombiner

- Intermittent operation of fan cooler(s) as necessary to control

- Containment pressure

- Steam condensation rate, humidity

- Recombiner/fan cooler as possible ignition source

- After all fan coolers are stopped, to what concentration must hydrogen be reduced by operation of the recombiner before a fan cooler can safely be restarted

- Contingency for unavailability of hydrogen recombiner - containment hydrogen purge system

- Knowledge of criteria for applying HLA (conditions of applicability): When the panel members evaluate the hydrogen purge contingency, do they consider the following points?

- Pre-calculation of fission-product release required to obtain a given reduction in containment hydrogen concentration

- Ability to secure purge (not a hardened vent path)

- Local radiation dose rates along the purge vent path

- Equipment accessibility

- Control room habitability

- Possibility of filtering the hydrogen purge release

- Risk versus benefit of hydrogen purge, as related to containment integrity

- Knowledge of limits of applicability for EOPs: How do the panel members reconcile the apparent conflict between the procedural guidance of ECA- 0.2 and the obvious exigencies of the situation?

\section{Control:}

- Ability to coordinate actions

- Improper coordination of hydrogen reduction and reduction of containment pressure/humidity could result in hydrogen ignition

- If fission products are deliberately released from containment via hydrogen purge system, extensive coordination with off-site management will be required

- Ability to determine that conditions for action have been met-account for changes in plant state, if any

- Ability to determine degree of action (for tuning type actions - how much): What criteria would the panel members establish for executing the plan?

- If (at some time after the fan coolers are stopped) containment pressure starts to increase, how low does the hydrogen concentration have to be before the fan coolers can be restarted?

- How high can containment pressure be allowed to go without running a fan cooler?

- If a fan cooler is restarted, how much can containment pressure be reduced (and how much steam can be condensed) before hydrogen combustion due to inerting again becomes a danger? 
PWR-3: Deliberate Flooding of Reactor Cavity

Warning:

This scenario is intended to be used as a tool or exercise for generating a response from a small accidentmanagement team (or panel) to a hypothetical severe accident. The function of this accident scenario is to present a situation that pushes the team outside of the standard, well-rehearsed procedures for addressing abnormal plant conditions. To achieve this, we created scenarios with difficult choices, goal conflicts, or unique situations that present significant challenges to the team's decision-making process.

Further, to create the necessary severe accident conditions in this scenario, it was necessary to postulate numerous traumas, equipment failures, and at times, operator errors. The use of multiple failures was required to thwart the safety and defense-in-depth design employed in nuclear power plants and reduce the effectiveness of current emergency operating procedures (EOPs).

The boundary conditions of the scenario were constructed for the sole purpose of maintaining a difficult decisionmaking situation for as long as possible. Therefore, it should not be construed that these scenarios are likely events, or even the most likely severe accident sequences. The probability of these scenarios occurring is within, or beyond, the range of traditional IPE core-damage frequencies.

\section{Scenario Objective:}

The objectives of this scenario are as follows:

- To place the panel members in a situation in which the core is known to be severely damaged already and in which failure of the reactor vessel might be imminent.

- To force the panel members to decide whether to flood the reactor cavity deliberately as a means of mitigating the consequences of reactor vessel failure.

- To present technical problems to the flooding of the reactor cavity with borated water that force the panel members to devise a solution that is not available in current procedures.

\section{Scenario Assumptions:}

These assumptions are for the presenter and are not to be presented to the panel:

- SNUPPS plant; forced-maintenance outage following SGTR; reactor shutdown for 84 hours; had been operating at full power for 200 days prior to SGTR

- RCS boron concentration 2000 ppm

- Two trains of RHR operable in the shutdown cooling mode; one train in operation; RCS temperature, $90 \mathrm{~F}$

- Reactor vessel head closure bolts fully tensioned; reactor cavity seal is not installed

- Containment integrity set

- All steam generators in dry lay-up

- RCS vent path history:

- Both PORVs operable in the cold over-pressure protection (COP) mode

- In preparation for mid-loop operation and installation of SG nozzle dams, the PZR manway was removed to provide a large hot-side vent

- Then, because of the high decay heat rate, it was decided that a PZR safety valve should also be removed and the associated loop seal drained, prior to going to mid-loop 
- However, the mechanical maintenance personnel sent into the containment not only removed a PZR safety valve and drained the associated loop seal, they also reinstalled the PZR manway and torqued the closure bolts; they did not inform anyone that they had closed the PZR manway

- RCS drained to mid-loop elevation to permit removal of the manways on the primary side of the steam generators

- Nozzle dams installed in the hot and cold legs of all steam generators

- Primary-side manways reinstalled (but not torqued) on all steam generators except SG A (both manways are left open)

\section{Progression of Events:}

$0 \mathrm{~min}$. Before RCS water level can be raised back above mid-loop, earthquake of greater than SSE magnitude occurs

- Earthquake causes loss of all AC power and some damage to safeguards systems/components

- Instrument air line severed from the pneumatic actuator of one of the PZR PORVs

5 min. Site Emergency declared; containment evacuated except for certain mechanical maintenance personnel who were known to be in containment at time of seismic event; the control room operators direct these personnel to remain in containment long enough to close the cold-side manway on SG A; however, the maintenance personnel close the hot-side manway and exit containment

$10 \mathrm{~min}$. The TSC and the EOF were activated

$20 \mathrm{~min}$. Plant operators attempt local-manual opening of RWST-to-RHR-suction isolation valves (HV-8812A and HV-8812B), but neither valve will open

$30 \mathrm{~min}$. Water in the reactor vessel starts to boil

$35 \mathrm{~min}$. Control room operators observe increasing RCS pressure and determine that RCS is insufficiently vented; they attempt to open both PZR PORVs but only one opens

$40 \mathrm{~min}$. The operators establish a gravity feed-flow path from the RWST to the cold legs via an 8-inch recirculation line in the discharge of the RHR pumps/ heat exchangers (manual valve 8717; localmanual operation of MOVs HV-8716A/B)

$45 \mathrm{~min}$. The cold-leg nozzle dam in SG A fails; much liquid inventory lost from reactor vessel through the open cold-leg manway

$55 \mathrm{~min}$. Emergency classification upgraded to General Emergency

- Because of RCS pressurization, gravity feed from the RWST is ineffective in maintaining reactor vessel liquid inventory (losses due to steam flow out the PZR PORV and safety valve opening and due to liquid leakage through the failed nozzle dam exceed the gravity-feed make-up capability)

$1 \mathrm{hr}$. Core completely uncovered

$5 \mathrm{hrs}$. Emergency diesel generator EDG B is repaired and started

- By this time, the core has slumped and $2 / 3$ of the core material has relocated to the lower plenum of the reactor vessel

- The relocated corium is not in a coolable configuration

- Operators take actions consistent with ARG-1

- Hot-leg injection via SI pump B

- Containment fan cooler units (2)

- ESW pump (1)

- CCW pump (1)

- Hydrogen mixing fans (2)

- Instrument air compressor (1)

\section{Other Notes:}

- This scenario does not involve inerting of the containment atmosphere by large quantities of steam. The scenario initiates from mid-loop operation when coolant inventory and RCS pressure and temperature are 
minimal. Thus, there is no danger of de-inerting the containment by running fan coolers or initiating containment spray. However, detonable or burnable mixtures of hydrogen and air may already exist.

- During the loss of all AC power, over-pressurizing the VCT to inject via the charging flow path is not a workable strategy because no pumps are available to make-up to a pressurized VCT.

\section{Statement for Panel:}

The plant had been forced into a maintenance outage by a SGTR. The reactor had been shutdown for 84 hours following a 200-day run at full power. The RCS boron concentration was $2000 \mathrm{ppm}$. Two trains of RHR were operable in the shutdown cooling mode; one train was in operation. RCS temperature was $90 \mathrm{~F}$.

The reactor vessel head closure studs were fully tensioned. The reactor cavity seal was not installed. Containment integrity was set. All steam generators were in dry lay-up.

Both PZR PORVs were operable in the cold-overpressure protection (COP) mode. In preparation for mid-loop operation and the installation of SG nozzle dams, the PZR manway was removed to provide a large hot-side vent. Then, because of the high decay heat rate, it was decided that a PZR safety valve should also be removed and the associated loop seal drained, prior to going to mid-loop.

However, the mechanical maintenance personnel sent into the containment not only removed a PZR safety valve and drained the associated loop seal, they also re-installed the PZR manway and torqued the closure bolts. They did not inform anyone that they had closed the PZR manway.

The RCS was drained to the mid-loop elevation to permit removal of the manways on the primary side of the steam generators. Nozzle dams were installed in the hot and cold legs of all steam generators. The primary-side manways were re-installed (but not torqued) on all steam generators except for SG A, on which both manways were left open.

Before reactor vessel water level could be raised back above the mid-loop elevation, an earthquake of greater than SSE magnitude occurred. The earthquake caused loss of all AC power and some damage to safeguards systems/ components. A Site Emergency was declared. The instrument air lines were severed from the pneumatic actuator of one of the PZR PORVs.

The control room operators ordered all personnel to evacuate containment, except for certain mechanical maintenance personnel who were known to be in containment at time of the seismic event. The control room operators directed these personnel to remain in containment long enough to close the cold-side manway on SG A. However, the maintenance personnel closed the hot-side manway and exited containment.

The TSC and the EOF were activated. The plant operators attempted local-manual opening of RWST-to-RHRsuction isolation valves (HV-8812A and $\mathrm{HV}-8812 \mathrm{~B})$, but neither valve would open.

Thirty minutes into the event, water in the reactor vessel started to boil. The control room operators observed the increasing RCS pressure and determined that the RCS was insufficiently vented. They attempted to open both PZR PORVs but only one opened. The operators established a gravity feed flow path from the RWST to the cold legs via an 8-inch recirculation line in the discharge of the RHR pumps/ heat exchangers (manual valve 8717; local-manual operation of MOVs HV-8716A/B).

Forty-five minutes into the event, the cold-leg nozzle dam in SG A failed. Much liquid inventory was lost from the reactor vessel through the open cold-leg manway. The emergency classification was upgraded to that of General Emergency. 
Because of RCS pressurization, gravity feed from the RWST was ineffective in maintaining reactor vessel liquid inventory. Losses due to steam flow out the PZR PORV and safety valve opening and due to liquid leakage through the failed nozzle dam exceeded the gravity feed make-up capability.

At one hour into the event, the core became completely uncovered. During the next four hours, the core was severely damaged.

At five hours into the event, emergency diesel generator (EDG) B is repaired and started. The control room operators take actions consistent with ARG-1 and start the following equipment:

- Hot-leg injection via SI pump B

- Containment fan cooler units (2)

- ESW pump (1)

- CCW pump (1)

- Hydrogen mixing fans (2)

- Instrument air compressor (1)

\section{Recommendation:}

Five hours and twenty minutes into the event, an engineer in the TSC makes the following recommendation: "Even though high-head hot-leg injection has been established, we can't be sure that the core is being adequately cooled. Some of the core is bound to have relocated to the vessel lower plenum. A crust of re-solidified corium could prevent the SI flow from reaching the molten material. The molten corium beneath the crust could attack the lower vessel head and penetrations."

"If the reactor vessel fails, we had better have water in the reactor cavity to quench the corium and prevent it from attacking the basemat. I suggest that we initiate containment spray in order to flood the reactor cavity up to the top of the lower vessel head. This might even prevent failure of the reactor vessel."

\section{Initial Questions to Panel:}

- Should this recommendation be implemented? Why or why not?

- If the vessel fails and corium pours into the flooded reactor cavity, will there be a steam explosion? Could the steam explosion disperse the cavity water and corium into the containment proper? If such a dispersal occurred, would it be good or bad?

- Would it be better to wait until after the vessel fails before flooding the cavity?

- Will the method proposed be effective in flooding the reactor cavity?

- Is there enough water in the RWST to achieve flooding of the cavity up to the top of the lower vessel head?

- As the RWST is depleted, would you attempt to transfer to a recirculation lineup via the RHR system?

- Would you modify the recommendation? If so, how?

- How much water in the cavity will start making a difference? How much is needed to quench the pour?

- What effects will wetting the lower vessel head have on vessel integrity?

- How long will it take to flood the cavity to the top of the lower vessel head?

- If the containment is flooded sufficiently to flood the reactor cavity, will instrumentation needed for accident management be immersed? If so, will the instrumentation fail?

- Should you spray into the containment at the maximum rate? Spray at the maximum rate initially and then throttle spray? 


\section{Background Material for Conducting Exercise:}

Current plant conditions, 20 minutes after restoration of power to safeguards bus "B", are as follows:

- RCS pressure: 86 psig, stable

- RCS hot-leg temperature: $700 \mathrm{~F}$, pegged high

- RVLIS static range: $120 \%$, pegged high

- Core-exit thermocouple temperature (avg): $0 \mathrm{~F}$

- Containment pressure: 0.5 psig, slowly decreasing

- Containment temperature: $130 \mathrm{~F}$, slowly decreasing

- Containment humidity: $60 \%$

- Containment radiation:

- Air particulate activity detector

- Gaseous activity detector

- Containment (high-range) area radiation monitor(s)

- Containment hydrogen concentration: $7 \%$ in dry air

- AC emergency buses: 4-kV bus "B" energized

- AC service buses: all de-energized

- Emergency classification level: General Emergency

- Equipment in "pull-to-lock"

- Motor-driven AFW pump "B"

- All safeguards train "A" equipment

- Instrument tunnel sump level: $100 \%$

- Containment normal sump level: $100 \%$

- Recirculation sump level: $8.2 \mathrm{ft}$

- RWST level: $94 \%$

- SI pump B flow rate: $615 \mathrm{gpm}$

- CCP B flow rate: $434 \mathrm{gpm}$

- Assumptions: SGs in dry lay-up; One PZR safety valve removed; RCS drained to mid-loop; Nozzle dams in hot and cold legs of all SGs; Earthquake greater than SSE; Loss of all AC power and pumped injection; Loss of one PZR PORV; inadequate hot-side vent; Coolant ejected via failed nozzle dam on cold-leg side of SG A and open primary manway; Limited gravity feed from RWST; Fuel melt and relocation into noncoolable geometry; Establishment of high-head hot leg injection

\section{Connections to Decision-Making Model:}

\section{Monitor/Detect:}

- Ability to obtain critical information on core status

- Important information is not instrumented, viz., whether core has relocated to lower vessel head

\section{Interpret Current State:}

- Ability to determine existing faults; core, vessel, containment status: Do the panel members agree with the TSC engineer's assessment of likely core relocation to the lower vessel head? Given the coolant ejection from mid-loop conditions and the amount of intervening time, it is likely that the core

- Boiled dry

- Heated up

- Melted and slumped to the lower core plate

- Melted through the lower core plate and relocated to the lower head

Do the panel members perceive any merit in the TSC engineer's concern about a non-coolable corium configuration or do they reject it out of hand? 
Do the panel members consider the following current plant indications to confirm/ disconfirm the TSC engineer's assessment of the core damage state?

- RCS hot-leg temperature pegged high,

- and CET avg temperature at $0 \mathrm{~F}$,

- while total SI flow rate has been greater than $1000 \mathrm{gpm}$ of subcooled fluid for 20 minutes,

- and RVLIS static range is pegged high.

These indications are consistent with RTD and thermocouple failure (open circuits) in a previously hightemperature environment that has been mitigated subsequent to the instrument failure. The containment hydrogen concentration of $7 \%$ is indicative of severe core damage, especially in light of the small inventory of water (due to the specific history of this event) that was available for the zirc-steam reaction.

\section{Plan:}

- Knowledge of criteria for applying HLA (conditions of applicability): Do the panel members:

- Try to determine the possible core damage state by considering the entire history of the event?

- Realize that, given the history of this event, vessel failure could be imminent?

- Consider the benefits of having water in the cavity at the time of vessel failure (that it is better for the corium to pour into a deep pool of water than onto dry concrete)?

- Appreciate the need for immediate initiation of actions to flood the reactor cavity?

- Appreciate that it will take a long time to flood the cavity and that, if there is any chance to get the cavity flooded before the vessel fails, they must initiate flooding ASAP

- Realize that no matter when the vessel fails, even if it fails before they get the cavity flooded, they will still have to cool the molten corium that escapes and the sooner they get started on the flooding the better

- Realize that if the vessel fails before the cavity is even partially flooded, it might be possible to pour water onto the escaped corium by continuing to inject water into the RCS - some of the injected water may spill through the vessel failure onto the escaped corium?

- Evaluate the feasibility of flooding the cavity by containment spray or some alternative method?

- Quantity of water that must be sprayed to flood the recirc sump(s) and the basement sufficiently to flood the reactor cavity

- Sufficient quantity of water available; impact on RWST depletion and SI; recirc

- Instrumentation that could be immersed; if immersed, will it fail and how soon

- Evaluate the need for flooding the cavity all the way to the top of the lower vessel head

- How deep must the pool of water in the cavity be to quench the pour, to break up the pour into coolable pieces

- Effect of wetting lower vessel head on vessel integrity

- Could it induce failure?

- Could it remove sufficient heat to prevent vessel failure?

- Knowledge of specific plant systems required for implementing plan or procedure: Do the panel members work out an efficient method for performing the flooding?

- Depending on the containment geometry, the cavity flood rate may be less than the containment spray rate-this would result in unnecessary accumulation of water in the containment basement with the attendant potential for flooding of instrumentation

- The most efficient method might be to spray at the maximum rate until the recirc sumps and basement are sufficiently flooded to begin flooding of the cavity-and then throttle the spray flow rate (reduce to one pump) to something approximating the likely cavity flood rate

- This method would conserve RWST inventory and delay the need to transfer to recirc

\section{Feedback:}

- Ability to determine that control action produced desired effect: Do the panel members identify a method of determining that the cavity is indeed flooding and to what depth? 
PWR-4: Early RCS Depressurization

\section{Warning:}

This scenario is intended to be used as a tool or exercise for generating a response from a small accidentmanagement team (or panel) to a hypothetical severe accident. The function of this accident scenario is to present a situation that pushes the team outside of the standard, well-rehearsed procedures for addressing abnormal plant conditions. To achieve this, we created scenarios with difficult choices, goal conflicts, or unique situations that present significant challenges to the team's decision-making process.

Further, to create the necessary severe accident conditions in this scenario, it was necessary to postulate numerous traumas, equipment failures, and at times, operator errors. The use of multiple failures was required to thwart the safety and defense-in-depth design employed in nuclear power plants and reduce the effectiveness of current emergency operating procedures (EOPs).

The boundary conditions of the scenario were constructed for the sole purpose of maintaining a difficult decisionmaking situation for as long as possible. Therefore, it should not be construed that these scenarios are likely events, or even the most likely severe accident sequences. The probability of these scenarios occurring is within, or beyond, the range of traditional IPE core-damage frequencies.

\section{Scenario Objective:}

The objectives of this scenario are as follows:

- To place the panel members in a situation that has an extremely high probability of leading to severe core damage and reactor vessel failure-loss of all $A C$, loss of all auxiliary (emergency) feed, and loss of feedinduced LOCA.

- To force the panel members to decide whether to deliberately depressurize the RCS early in the accident as a means of preventing or mitigating direct containment heating $(\mathrm{DCH})$; to see if the panel members realize that late depressurization is preferred over early.

- To present technical problems associated with early RCS depressurization (as an anti-DCH measure) that force the panel members to devise a solution that is not available in current procedures.

\section{Scenario Assumptions:}

These assumptions are for the presenter and are not to be presented to the panel:

- SNUPPS plant; late in fuel cycle; critical boron concentration at hot full power was 200 ppm; plant had operated at full power for greater than 200 days

\section{Progression of Events:}

0 min. Earthquake of magnitude greater than the SSE causes loss of all AC power and some damage to safeguards systems/components

- Steam-driven AFW pump damaged; cannot be started

- SG PORVs fail closed; cannot be opened even by local-manual operation

- Automatic reactor trip; SG safety valves open 12 sec after reactor trip

- Control room operators enter ECA-0.0 and declare General Emergency; TSC and EOF activated

$32 \mathrm{~min}$. All SGs dry out 
35 min. Both PZR PORVs open

- RCP seals deteriorate and develop leakage to the containment; the seal leakage rate reaches $20 \mathrm{gpm}$ per pump

- All efforts to restore AC power and aux feed fail; damage to electric plant and aux feed system so severe that earliest estimate for repairs is 12-16 hours

- Because they cannot establish any feed flow to the SGs, the operators do not depressurize the SGs

- ECA-0.0 leaves the operators performing an intentional do-loop in which they monitor RCS conditions and try to accomplish the following:

- Restore power to an AC emergency bus

- Establish a secondary heat sink (total aux feed flow to all intact SGs greater than $260 \mathrm{klbm} / \mathrm{hr}$ or NR level in at least one intact SG greater than $4 \%$ ) [for SNUPPS, the operators are not supposed to commence secondary depressurization until after NR level in at least one intact SG is greater than $4 \%$

\section{Statement for Panel:}

The plant had been operating at $100 \%$ power for greater than 200 days. The critical boron concentration at hot full power was $200 \mathrm{ppm}$. About an hour ago, an earthquake of greater than SSE magnitude caused loss of all AC power and some damage to safeguards systems/ components.

The steam-driven AFW pump was damaged; it cannot be started. The SG PORVs failed closed; they cannot be opened even by local-manual operation.

The reactor tripped automatically. The SG safety valves opened 12 seconds after the reactor trip. The control room operators entered ECA- -0.0 and declared a General Emergency. The TSC and EOF were activated.

At 32 minutes into the event, all SGs dried out. At 35 minutes into the event, both PRZ PORVs opened and started to cycle RCS pressure about their open and close setpoints.

The RCP seals deteriorated and developed leakage to the containment; the seal leakage rate has reached $20 \mathrm{gpm}$ per pump. All efforts to restore $\mathrm{AC}$ power and aux feed have failed. Damage to the electric plant and to the aux feed system is so severe that the earliest estimate for repairs is $12-16$ hours.

Because they cannot establish any feed flow to the SGs, the operators have not depressurized the SGs. ECA-0.0 has left the operators performing an intentional do-loop in which they monitor RCS conditions and try to accomplish the following:

- Restore power to an AC emergency bus

- Establish a secondary heat sink (total aux feed flow to all intact SGs greater than $260 \mathrm{klbm} / \mathrm{hr}$ or NR level in at least one intact SG greater than $4 \%$ )

\section{Recommendation:}

One hour into the event, an engineer in the TSC makes the following recommendation:

"We are in a situation that is bound to lead to severe core damage unless we regain a vital bus or a secondary heat sink. It's likely to be several hours before we regain either, during which time the core will melt. In fact, it is possible that the reactor vessel will fail before we get a vital bus back." 
"I think it is imperative that we take action now to depressurize the RCS. If the reactor vessel fails while the RCS is still at a high pressure, molten corium could be dispersed into the containment atmosphere beyond the cavity and lead to direct containment heating. This could challenge containment integrity."

'We don't have a secondary heat sink. We are loosing RCS inventory through the RCP seals and the PZR PORVs. We might as well latch the PORVs open and depressurize the RCS. There is only a limited supply of back-up instrument air pressure available. When it is depleted by cycling the PZR PORVs, we won't be able to latch the PORVs open, should we later decide that we need to."

"We should latch open both PZR PORVs now, while we can. We must make sure that the RCS is depressurized before the reactor vessel fails."

\section{Initial Questions to Panel:}

- Should this recommendation be implemented? Why or why not?

- How much must the RCS be depressurized to preclude DCH?

- Will latching the PZR PORVs open be effective in reducing RCS pressure the required amount?

- If the RCS is depressurized as proposed, will the core remain covered?

- Would it be better to depressurize the secondary side of the SGs, even though they have dried out? Is there a concern about SGTR?

- Would it be better to wait before depressurizing the RCS, for example, wait until CET temperature reaches 1200 F?

\section{Background Material for Conducting Exercise:}

Current Plant Conditions, one hour into the event, are as follows:

- RCS pressure: cycling between the PZR PORV opening and closing setpoints (2335 and 2315 psig)

- RCS hot-leg temperature: $658 \mathrm{~F}$, stable

- RVLIS static range: $80 \%$, decreasing

- Core exit thermocouple temperature (avg): $690 \mathrm{~F}$, stable

- Containment pressure: 0.5 psig, slowly increasing

- Containment temperature: $130 \mathrm{~F}$, slowly increasing

- Containment humidity: $95 \%$

- Containment radiation:

- Air particulate activity detector

- Gaseous activity detector

- Containment (high-range) area radiation monitor(s)

- Containment hydrogen concentration: $0.5 \%$ in dry air

- AC emergency buses: all de-energized

- AC service buses: all de-energized

- Emergency classification level: General Emergency

- Equipment in "pull-to-lock"

- All safeguards train "A" and train "B" equipment, except for ESW pumps A and B

- Instrument tunnel sump level: $100 \%$

- Containment normal sump level: $100 \%$

- Recirculation sump level: $8.2 \mathrm{ft}$

- RWST level: $98 \%$

- Assumptions: Earthquake greater than SSE; loss of all AC power; loss of pumped injection; loss of all normal and emergency feed, including steam-turbine-driven AFW pump; all SG PORVs stuck shut; loss of secondary heat sink; SG dryout; loss-of-feed-induced LOCA 
Connections to Decision-Making Model:

Monitor/Detect:

- Ability to determine that control action produced desired effect on key parameters: Do the panel members specify which RCS pressure indications to use during the depressurization?

Do they anticipate the RCS temperature response?

\section{Interpret Current State:}

- Ability to determine existing faults; safety function status; core, vessel, containment status; availability of safety systems

- Ability to develop complete and accurate mental representation of plant status: Do the panel members understand the following?:

- The core is about to start uncovering because of the loss of feed-induced LOCA that is in progress

- The RCS is saturated and core heat-up has not begun yet

- The TSC engineer is correct when he says that, given the estimated time for repairs, the core will melt and the vessel might fail before AFW or electrical power is restored

- The mechanism of $\mathrm{DCH}$ and the fact that $\mathrm{DCH}$ can be a threat to containment integrity, depending on the plant-specific containment geometry

\section{Determine Implications:}

- Ability to remove bad or irrelevant ideas from consideration: Do the panel members realize the following?:

- Although the exact magnitude of the threat posed by DCH to containment integrity is uncertain, it is advisable to preclude DCH by preventing high-pressure blowdown of the RCS

- Depressurization, early or late, might increase the risk of a steam explosion when the molten core relocates to the lower head because steam explosions are more likely at lower pressures

- In-vessel steam explosion is not expected to be energetic enough to breach the RPV

- It is better not to depressurize the RCS until after the core has started to uncover

- Early depressurization causes more inventory to be lost sooner and accelerates the onset of core heatup

- Late depressurization causes less inventory to be lost early in the transient and postpones core heat-up

- Given the estimated time for repairs to the AFW and plant electric systems, the core is certainly doomed and vessel integrity is probably doomed. Therefore, containment integrity must be the highest priority and the threat from DCH must be addressed.

Do the panel members consider the following?:

- What if PZR PORV(s) fail(s) closed, open?

- Will secondary depressurization prevent high-pressure ejection of molten corium; would it raise a concern about SGTR?

- Whether the plant-specific containment geometry obviates the concern about $\mathrm{DCH}$.

Plan:

- Knowledge of criteria for applying HLA (conditions of applicability): Do the panel members consider the following?:

- Early depressurization takes longer than late because a mixture of liquid and vapor is blown off during early depressurization whereas only vapor is blown off during late depressurization

- Because early depressurization takes comparatively longer, the temperature of some of the fuel rods exceeds the threshold for oxidation of zircaloy before the accumulators inject. Thus, for early 
depressurization, accumulator injection causes significant cladding oxidation and relocation and is not effective in controlling further core damage.

- Will they be able to depressurize the RCS sufficiently via the PZR PORVs to mitigate DCH?

- Is there sufficient instrument air capacity (continuous supply of air needed to hold valves open)?

- Sufficient electrical power?

- Are both PORV block MOVs open?

- To what value must RCS pressure be reduced? (to within about 145 psi of containment pressure)

- Possible downside consequences (e.g., hydrogen generation and steam explosion)

- Early depressurization could lead to PZR surge-line failure prior to RPV failure, while the accumulators are injecting

- Late depressurization maximizes the time available to recover $A C$ power and AFW

- Knowledge of limits of applicability for EOPs: Do the panel members recognize the following?:

- If they remain in the intentional do-loop prescribed by ECA-0.0, the core will melt and the vessel might fail

- For the prevailing plant conditions, ECA-0.0 provides no transition to FRGs that would direct the operators to depressurize the RCS (or the secondary)

- Knowledge of specific plant systems required for implementing plan or procedure: Do the panel members consider the following actions?:

- Over-riding containment isolation for instrument air to the PZR PORVs AND

- Shunting air from other uses/reservoirs/accumulators OR

- Connecting nitrogen or other compressed gas system or bottles to supply PZR PORV actuators

- Cycling of the PORVs, while waiting for late depressurization criteria to be reached, consumes more instrument air than does latching them in the open position

If the panel members realize that late depressurization is preferred, do they consider the following actions?:

- Performing a series of shallow depressurizations to minimize PORV cycling and thereby postpone exhaustion of the instrument air supply

- What initiation criteria will be used for late depressurization?

\section{Control:}

- Ability to determine when specific actions should be taken: Do the panel members realize that, if they opt for late depressurization, they must initiate depressurization within a few minutes of CET temperature reaching $1200 \mathrm{~F}$, or else unacceptably high clad/fuel temperatures will result? 
PWR-5: Natural Circulation versus RHR Cooling

\section{Warning:}

This scenario is intended to be used as a tool or exercise for generating a response from a small accidentmanagement team (or panel) to a hypothetical severe accident. The function of this accident scenario is to present a situation that pushes the team outside of the standard, well-rehearsed procedures for addressing abnormal plant conditions. To achieve this, we created scenarios with difficult choices, goal conflicts, or unique situations that present significant challenges to the team's decision-making process.

Further, to create the necessary severe accident conditions in this scenario, it was necessary to postulate numerous traumas, equipment failures, and at times, operator errors. The use of multiple failures was required to thwart the safety and defense-in-depth design employed in nuclear power plants and reduce the effectiveness of current emergency operating procedures (EOPs).

The boundary conditions of the scenario were constructed for the sole purpose of maintaining a difficult decisionmaking situation for as long as possible. Therefore, it should not be construed that these scenarios are likely events, or even the most likely severe accident sequences. The probability of these scenarios occurring is within, or beyond, the range of traditional IPE core-damage frequencies.

\section{Scenario Objective:}

The objectives of this scenario are as follows:

- To place the panel members in a situation in which the core has overheated and for which the guidance provided in the existing emergency procedures may be perceived as counter-productive

- To force the panel members to decide whether to transfer to cold leg recirculation on RHR or to try to establish RCS natural circulation cooling

- To present perceived hazards associated with transfer to cold-leg recirculation and to present a proposal for restoring the secondary heat sink to establish natural circulation. Evaluating the perceived hazards of recirculation, assessing the technical feasibility of restoring the secondary heat sink, and estimating the likelihood of establishing RCS natural circulation should force the panel members to exercise cognitive skills to supplement current procedures.

\section{Scenario Assumptions:}

These assumptions are for the presenter and are not to be presented to the panel:

- SNUPPS plant; late in fuel cycle; critical boron concentration at hot full power was 200 ppm; plant had operated at full power for greater than 200 days

\section{Progression of Events:}

0 min. Earthquake of magnitude greater than the SSE causes loss of all AC power and some damage to safeguards systems/ components

- Motor-driven and steam-driven AFW pumps damaged; none can be started

- RWST damaged such that, over a period of 100 minutes, inventory is lost due to leakage down to the tank level that corresponds to $45 \%$ of the inventory required by Technical Specifications

- Automatic reactor trip; SG safety valves open $12 \mathrm{sec}$ after reactor trip

- Control room operators enter ECA-0.0 and declare General Emergency; TSC and EOF activated 
32 min. All SGs dry out

35 min. Both PZR PORVs open

- RCP seals deteriorate and develop leakage to the containment; the seal leakage rate reaches $20 \mathrm{gpm}$ per pump

$100 \mathrm{~min}$. Emergency diesel generator (EDG) B is repaired and started

- All efforts to restore off-site power and aux feed fail; damage to off-site transmission network, to EDG $A$, to on-site train A emergency AC distribution system, and to aux feed system so severe that earliest estimate for repairs is 20-24 hours

- When safeguards bus B is energized, the PZR PORVs have closed and the following indications exist:

- CET temperature (avg): $2900 \mathrm{~F}$, increasing

- RVLIS static range: $15 \%$, decreasing

- RCS pressure: 1900 psig, decreasing

- RCS hot leg temperature: $700 \mathrm{~F}$

- Control room operators

- Manually actuate SI and load ECCS pumps onto safeguards bus B

- Transition to FR-C.1; however,

- Because they cannot establish any feed flow to the SGs, the operators do not depressurize the SGs

- Because they cannot restore off-site power, the operators cannot start RCPs

110 min. Control room operators initiate RCS bleed and feed per FR-H.1

- Effects of RCS feed and bleed

- SI flow eventually cools fuel clad

- CET temperatures fall

- Eventually, RCS pressure decreases and SI flow increases

- Initiation of SI flow causes the following:

- Some overheated cladding shatters

- Additional hydrogen is produced for a brief period of time, until cladding temperatures are reduced

- Progression of core damage stops with

- Core in coolable geometry

- Significant amount of fission products released to RCS and to containment

$150 \mathrm{~min}$. Plant conditions as follows:

- Instrumentation indications:

- RCS pressure: 351 psig, decreasing

- RCS hot leg temperature: $390 \mathrm{~F}$, decreasing

- RVLIS static range: $100 \%$

- CET temperature (avg): $400 \mathrm{~F}$, decreasing

- Containment pressure: 1.5 psig, slowly decreasing

- Containment temperature: $150 \mathrm{~F}$, slowly decreasing

- Containment humidity: 50\%, decreasing

- Containment radiation:

- Air particulate activity detector

- Gaseous activity detector

- Containment (high-range) area radiation monitor(s)

- Containment hydrogen concentration: $3.5 \%$ in dry air

- Instrument tunnel sump level: $100 \%$

- Containment normal sump level: $100 \%$

- Recirculation sump level: $8.2 \mathrm{ft}$

- RWST level: $35 \%$ 
- CCP B flow rate (BIT flow): 434 gpm

- SI pump B flow rate: $615 \mathrm{gpm}$

- $A C$ emergency buses: one energized

- AC service buses: all de-energized

- Emergency classification level: General Emergency

- Equipment in "pull-to-lock"

- All safeguards train "A" equipment, except for ESW pump A

- Procedure \& step in effect: FR-H.1, intentional do-loop wherein secondary heat sink must be established before RCS bleed and feed can be reduced/terminated

- CDA indicates severe fuel overheat

\section{Statement for Panel:}

The plant had been operating at $100 \%$ power for greater than 200 days. The critical boron concentration at hot full power was $200 \mathrm{ppm}$. About two and a half hours ago, an earthquake of greater than SSE magnitude caused loss of all AC power and some damage to safeguards systems/ components.

The motor-driven and steam-driven AFW pumps were damaged; none of the AFW pumps can be started. The RWST was damaged and lost inventory due to leakage down to a tank level that corresponds to $45 \%$ of the inventory required by Technical Specifications. Below this level, the tank is intact.

The reactor tripped automatically. The SG safety valves opened 12 seconds after the reactor trip. The control room operators entered ECA- 0.0 and declared a General Emergency. The TSC and EOF were activated.

At 32 minutes into the event, all SGs dried out. At 35 minutes into the event, both PRZ PORVs opened and started to cycle RCS pressure about their open and close setpoints. The RCP seals deteriorated and developed leakage to the containment; the seal leakage rate has reached $20 \mathrm{gpm}$ per pump.

At 100 minutes into the event, emergency diesel generator (EDG) B was repaired and started. All efforts to restore off-site power and aux feed have failed. Damage to the off-site transmission network, to EDG A, to the on-site train A emergency AC distribution system, and to the aux feed system is so severe that the earliest estimate for repairs is 20-24 hours.

When safeguards bus B was re-energized, the PZR PORVs had closed and the following indications existed:

- CET temperature (avg): $2900 \mathrm{~F}$, increasing

- RVLIS static range: $15 \%$, decreasing

- RCS pressure: 1900 psig, decreasing

- RCS hot leg temperature: $700 \mathrm{~F}$

As soon as the bus was energized, the control room operators manually actuated SI and loaded ECCS pumps onto EDG B. They transitioned to FR-C.1. Because they could not establish any feed flow to the SGs, the operators did not depressurize the SGs. Because they could not restore off-site power, the operators could not start RCPs.

At 110 minutes into the event, the control room operators initiated RCS bleed and feed per FR-H.1. Over a period of forty minutes, RCS pressure decreased, SI flow increased, and CET temperatures fell.

\section{Recommendation:}

Two hours and thirty minutes into the event, an engineer in the TSC makes the following recommendation: "RWST level is almost down to the point where procedure FR-H.1 will direct us to transfer from cold-leg injection to cold-leg recirculation. However, I recommend that we do NOT transfer to recirculation because doing so will 
result in large amounts of radioactive material being circulated outside of containment, due to the degraded state of the core. This will cause extremely high radiation levels inside of the auxiliary building and increase the potential for off-site releases via containment bypass. If core debris are circulated through the RHR pump, the pump impeller or seals might fail. Then, we'll loose core cooling again and release radioactive material to the auxiliary building."

"I think that we should leave the ECCS aligned for cold-leg injection and use the remaining RWST inventory to buy us some more time to establish a secondary heat sink. We can jury rig a connection from the fire-fighting system to the SG feedwater system and run the diesel-driven fire water pump to provide feed flow. We can use the SG PORVs to depressurize the SGs to atmospheric and thereby maximize feed flow from the fire water pump. Re-establishing the secondary heat sink will allow us to restore natural circulation in the RCS. Removing decay heat via RCS natural circulation and primary-to-secondary heat transfer will avoid the potential hazards associated with cold-leg recirculation via the RHR system."

\section{Initial Questions to Panel:}

- Should this recommendation be implemented? Why or why not?

- Are the perceived hazards associated with cold-leg recirculation on RHR cooling valid? Do they justify leaving the ECCS aligned for injection while attempting to restore the secondary heat sink?

- Is the proposed method of restoring the secondary heat sink technically feasible?

- Even if the secondary heat sink is successfully restored, is it likely that RCS natural circulation can be established? Is there a concern about hydrogen in the SG U-tubes?

\section{Background Material for Conducting Exercise:}

Current plant conditions, two and one half hours into the event, are as follows:

- Instrumentation indications:

- RCS pressure: 351 psig, decreasing

- RCS hot-leg temperature: $390 \mathrm{~F}$, decreasing

- RVLIS static range: $100 \%$

- CET temperature (avg): $400 \mathrm{~F}$, decreasing

- Containment pressure: 1.5 psig, slowly decreasing

- Containment temperature: $150 \mathrm{~F}$, slowly decreasing

- Containment humidity: 50\%, decreasing

- Containment radiation:

- Air particulate activity detector

- Gaseous activity detector

- Containment (high-range) area radiation monitor(s)

- Containment hydrogen concentration: $3.5 \%$ in dry air

- Instrument tunnel sump level: $100 \%$

- Containment normal sump level: $100 \%$

- Recirculation sump level: $8.2 \mathrm{ft}$

- RWST level: $35 \%$

- CCP B flow rate (BIT flow): 434 gpm

- SI pump B flow rate: $615 \mathrm{gpm}$

- AC emergency buses: one energized

- AC service buses: all de-energized

- Emergency classification level: General Emergency

- Equipment in "pull-to-lock"

- All safeguards train "A" equipment, except for ESW pump A 
- Procedure \& step in effect: FR-H.1, intentional do-loop wherein secondary heat sink must be established before RCS bleed and feed can be reduced/terminated

- CDA indicates severe fuel overheat

- Assumptions: Earthquake greater than SSE; loss of all AC power; loss of pumped injection; loss of all normal and emergency feed, including steam-turbine-driven AFW pump; SG dryout; loss of feed-induced LOCA; only one AC emergency bus can be recovered, off-site power remains unavailable; cladding failure

\section{Connections to Decision-Making Model:}

\section{Interpret Current State:}

- Ability to develop complete and accurate mental representation of plant status: Do the panel members understand that it is virtually certain that extensive cladding failure has occurred?

- Core is cool now (current plant status); but event history (Background) reveals the following:

- Core completely uncovered (RVLIS static range: $15 \%$, decreasing)

- Elevated CET temperatures (2900F, increasing)

- Zirc/steam reaction oxidizes and embrittles cladding

- Initiation of SI flow under these conditions shatters much cladding

- CDA confirms extensive cladding failure

Do the panel members understand that during the period of core uncovery fission products were released into the RCS and into the containment?

- CDA could confirm the extent of fission product release

Do the panel members understand that failure or bypass of the containment boundary under the present conditions could result in a significant release of fission products to the environment?

\section{Determine Implications:}

- Ability to remove bad or irrelevant ideas from consideration: Do the panel members try to determine how much longer they can remain on injection?

- Injection rates are known

- RWST level is known

Do the panel members consider the basis for the transfer-to-recirc criterion (RWST level indication of $33 \%$, decreasing)?

- The bottom $33 \%$ is reserved for containment spray

Do the panel members consider the following?:

- Containment pressure is 1.5 psig and decreasing

- Two containment fan coolers powered from safeguards bus B are running

- Containment spray won't be needed unless there is a hydrogen explosion, which is unlikely, given the current containment hydrogen concentration of $3.5 \%$ in dry air

- The current containment hydrogen concentration allows operation of the hydrogen recombiner without any further evaluation

Do the panel members consider the design basis for the ECCS piping and components and associated radiological shielding? Do they assess the dose rates likely to result as a consequence of transferring to recirc? Do the panel members consider the likelihood of containment bypass due to failure of the RHR or other ECCS system?

Plan:

- Knowledge of criteria for applying HLA (conditions of applicability): Do the panel members evaluate the following?:

- Whether or not the SGs can remove decay heat, given the current plant conditions 
- Will steaming occur?

- Is it enough just to remove decay heat or is it necessary to cooldown the RCS below 250-200 F?

- Plant-specific feasibility of jury rigging a connection from the fire-fighting system to the SG feedwater system

- Can the SGs be depressurized sufficiently via the SG PORVs to allow the fire water pump to inject into the SGs?

- The likelihood of establishing natural circulation in the RCS, given the presence of hydrogen and other noncondensibles in the RCS (hydrogen in the SG U-tubes)

- Do they use CDA to estimate the amount of hydrogen that may be in the RCS?

- Do they evaluate whether that amount of hydrogen is likely to interfere with natural circulation?

- Knowledge of limits of applicability for EOPs: Do the panel members consider the basis for the transfer-torecirc criterion (RWST level indication of $33 \%$, decreasing)?

- The bottom $33 \%$ is reserved for containment spray

- Knowledge of specific plant systems required for implementing plan or procedure: Do the panel members devise a procedure for cross-connecting the fire fighting and SG feedwater systems that addresses the following?:

- Specific connection points

- Method of connection

- Required pressure and flow-rate capacities

- Need to isolate parts of the fire-fighting system to ensure adequate pressure and flow for SGs

- Contingency plan for unisolating parts of fire fighting system in case of fire

Do the panel members devise a procedure for depressurizing the SGs via the SG PORVs that addresses the following?:

- To what pressure should the SGs be depressurized?

Do the panel members realize that the SGs must be depressurized to a pressure that is at least $50-100$ psi below RCS pressure before any pri-sec heat transfer can occur

- Can SG depressurization be performed remotely from the control room or will local manual operation be necessary?

- Status of instrument air supply

- If it has to be done locally, how will it be coordinated? 
PWR-6: Containment Spray versus ECCS Injection

\section{Warning:}

This scenario is intended to be used as a tool or exercise for generating a response from a small accidentmanagement team (or panel) to a hypothetical severe accident. The function of this accident scenario is to present a situation that pushes the team outside of the standard, well-rehearsed procedures for addressing abnormal plant conditions. To achieve this, we created scenarios with difficult choices, goal conflicts, or unique situations that present significant challenges to the team's decision-making process.

Further, to create the necessary severe accident conditions in this scenario, it was necessary to postulate numerous traumas, equipment failures, and at times, operator errors. The use of multiple failures was required to thwart the safety and defense-in-depth design employed in nuclear power plants and reduce the effectiveness of current emergency operating procedures (EOPs).

The boundary conditions of the scenario were constructed for the sole purpose of maintaining a difficult decisionmaking situation for as long as possible. Therefore, it should not be construed that these scenarios are likely events, or even the most likely severe accident sequences. The probability of these scenarios occurring is within, or beyond, the range of traditional IPE core-damage frequencies.

\section{Scenario Objective:}

The objectives of this scenario are as follows:

- To place the panel members in a situation in which the containment fan cooler units (CFCUs) and ECCS recirculation capability have been lost and in which RWST inventory is being rapidly depleted by containment spray, which actuated due to a pressure spike caused by a hydrogen burn following a period of severe core damage

- To force the panel members to decide whether sacrifice containment cooling by terminating sprays in order to preserve ECCS injection capability

- To present technical concerns involved in prioritizing core cooling and containment cooling that force the panel members to exercise cognitive skills to supplement current procedures.

\section{Scenario Assumptions:}

These assumptions are for the presenter and are not to be presented to the panel:

- SNUPPS plant; late in fuel cycle; critical boron concentration at hot full power was 200 ppm; plant had operated at full power for greater than 200 days

\section{Progression of Events:}

0 min. Earthquake of magnitude greater than the SSE causes 4-inch cold-leg break with coincident loss of all AC power and damage to safeguards systems

- Recirc-sump-to-RHR-suction isolation valves (EJ HIS-8811A and EJ HIS-8811B) will not open, even after electrical power is available (limit switch failure)

- Recirc-sump-to-containment-spray-suction isolation valves (EN HIS-1 and EN HIS-7) will not open, even after electrical power is available

- Common-mode failure (progressive loss of bearing lubrication-induced by seismic accelerations) leads to eventual failure (bearing seizure) of all four containment fan cooler units (CFCUs); CFCU 
failure occurs during a containment pressure spike caused by a hydrogen burn after AC emergency power is restored and after a period of running in the dense, moisture-laden containment atmosphere

- Manual reactor trip and automatic safety injection actuation signal

$12 \mathrm{~min}$. Accumulators inject $(720 \mathrm{sec})$ into the event

- RCP seals deteriorate and develop leakage to the containment; the seal leakage rate reaches $50 \mathrm{gpm}$ per pump and then decreases as RCS pressure decreases

$65 \mathrm{~min}$. Core uncovery begins

$94 \mathrm{~min}$. Core melt begins

$108 \mathrm{~min}$. Core slump begins

$120 \mathrm{~min}$. Off-site power restored to $4-\mathrm{kV}$ safeguards buses $\mathrm{A}$ and $\mathrm{B}$

- Safeguards equipment is manually loaded onto the re-energized buses, as follows:

- Essential service water pumps A and B

- Component cooling water pumps A and B

- RHR pumps A and B

- SI pumps $A$ and $B$

- CFCUs A, B, C, and D

- Hydrogen mixing fans $A$ and $B$

- Instrument air compressors $A$ and $B$

- Centrifugal charging pumps A and B

- Containment spray pumps A and B are placed in standby

- Motor-driven AFW pumps A and B are left in "pull-to-lock"

150 min. Hydrogen burn in containment

- Pressure spike of 35 psi (to 37 psig) actuates containment spray

- All 4 CFCUs fail

$210 \mathrm{~min}$. RWST level indication drops below the setpoint for automatic switchover to cold-leg recirculation

- Automatic switchover fails to occur

- Operators

- Determine that there is sufficient inventory in the containment recirculation sump to allow transfer to cold-leg recirculation

- Discover that the recirc-sump-to-RHR-suction isolation valves (EJ HIS-8811A and EJ HIS-8811B) will not open, even though electrical power is available

- Discover that the recirc-sump-to-containment- spray-suction isolation valves (EN HIS-1 and EN HIS7) will not open, even though electrical power is available

\section{Statement for Panel:}

The plant had been operating at $100 \%$ power for greater than 200 days. The critical boron concentration at hot full power was $200 \mathrm{ppm}$. About three and one half hours ago, an earthquake of magnitude greater than the SSE

occurred. The control room operators manually tripped the reactor. Safety injection actuated automatically. The earthquake caused a loss of all AC power and some damage to safeguards systems and components. 
The control room operators entered ECA-0.0 and declared a Site Emergency. The TSC and the EOF were activated. From RCS and containment indications, the operators determined that the RCS was not intact. The early onset of significant primary LOCA symptoms led the operators to conclude that the RCS pressure boundary had already been breached prior to and independent of the development of any RCP seal leakage.

Twenty minutes into the event, the emergency classification level was upgraded to that of General Emergency. In accordance with ECA-0.0, the operators depressurized the steam generators to 230 psig via manual operation of the PORVs. They verified containment isolation and containment ventilation isolation.

Plant operations, maintenance, and technical support personnel and off-site personnel engaged in coordinated efforts to start the emergency diesel generators (EDGs), to restore off-site power, and to conserve DC power supplies. However, because of the extensive repairs required, all AC buses remained de-energized for two hours.

During the time that the plant was without AC power, conditions in the RCS, core, and containment deteriorated. Core uncovery began at 65 minutes into the event, as indicated on the static range of RVLIS. At 75 minutes into the event, RCS pressure was 250 psig and RCS hot-leg temperature reached $700 \mathrm{~F}$. At 105 minutes into the event, core exit thermocouple temperatures reached $2300 \mathrm{~F}$.

At 120 minutes into the event, the following indications existed:

- RCS pressure: 200 psig and slowly decreasing

- RCS hot-leg temperature: $700 \mathrm{~F}$

- RVLIS static range: $10 \%$

- Core exit thermocouple temperature (avg): $3500 \mathrm{~F}$

- Containment pressure: 20 psig and slowly increasing

- Containment temperature: $185 \mathrm{~F}$ and slowly increasing

- Containment humidity: $100 \%$

- Containment radiation:

- Air particulate activity detector

- Gaseous activity detector

- Containment (high-range) area radiation monitor(s)

- Containment hydrogen concentration: $10 \%$ in dry air, increasing

Two hours into the event, off-site power was restored to all safeguards buses. The control room operators transitioned to ECA-0.2. They manually loaded the following safeguards equipment onto the re-energized buses:

- Essential service water pumps A and B

- Component cooling water pumps $A$ and $B$

- RHR pumps $A$ and $B$

- SI pumps $A$ and $B$

- CFCUs A, B, C, and D

- Hydrogen mixing fans $A$ and $B$

- Instrument air compressors $A$ and $B$

- Centrifugal charging pumps $A$ and $B$

The operators placed containment spray pumps A and B in standby and left motor-driven AFW pumps A and B in "pull-to-lock".

Two and one half hours into the event, there was a hydrogen burn in the containment atmosphere. The resulting pressure spike actuated containment spray. All four CFCUs stopped running at the time of the containment pressure spike. The control room operators have not been able to re-start any of the CFCUs. 
Three and one half hours into the event, as RWST level indication dropped below the setpoint for automatic switchover to cold-leg recirculation, the operators realized that switchover did not automatically occur. They determined that there was sufficient inventory in the containment recirculation sump to allow transfer to cold-leg recirculation. While attempting to implement ES-1.3, the operators discovered that the recirc-sump-to-RHRsuction isolation valves (EJ HIS-8811A and EJ HIS-8811B) would not open, even though electrical power was available.

The control room operators transitioned to ECA-1.1. They stopped containment spray pump A and placed it in standby. Because containment pressure had not yet been reduced to less than $3.5 \mathrm{psig}$, the operators left containment spray pump B running and tried to align it for recirculation. However, neither of the recirc-sump-tocontainment-spray-suction isolation valves (EN HIS-1 and EN HIS-7) will open, even though electrical power is available. The operators reduced safety injection to a single train of ECCS.

\section{Recommendation:}

Three hours and thirty-five minutes into the event, after learning that recirculation capability has been lost and that one containment spray pump is still running in the injection mode, an engineer in the TSC makes the following recommendation:

"I think we should stop containment spray pump B, even though containment pressure is still greater than 3.5 psig and no CFCUs are operable. Because we have been unable to transfer to cold-leg recirculation, it is imperative that we maintain cold-leg injection. The spray pump is depleting RWST inventory at a much faster rate than we can ever hope to make up. We must terminate spray to conserve RWST inventory and preserve injection. Besides, right now, we don't need the spray pump to preserve containment integrity."

\section{Initial Questions to Panel:}

- Should this recommendation be implemented? Why or why not?

- Given the conditions of this scenario, what is your evaluation of the threat to containment integrity if the recommendation of the engineer in the TSC is implemented?

- Assume that the recommendation is implemented and that RWST inventory is eventually depleted anyway. In such a case, it would be necessary to stop the ECCS and containment spray pumps. Would you start an RCP? Why or why not?

- Can you think of any methods of making up to the RWST at a rate greater than $120-200 \mathrm{gpm}$ ?

- Would it be feasible to restore recirculation capability by cross-connecting the discharge of one of the containment spray pumps with the discharge of one of the RHR pumps? If so, would you do it, given the conditions of this scenario?

\section{Background Material for Conducting Exercise:}

The current plant conditions, three hours and thirty-five minutes into the event, one hour and thirty-five minutes after restoration of AC power to the safeguards buses, and one hour and five minutes after the hydrogen burn, are as follows:

- RCS pressure: 150 psig and stable

- RCS hot-leg temperature: $700 \mathrm{~F}$

- RVLIS static range: $100 \%$

- Core exit thermocouple temperature (avg): $0 \mathrm{~F}$

- Containment pressure: 10 psig and slowly decreasing

- Containment temperature: $150 \mathrm{~F}$ and slowly decreasing

- Containment humidity: $50 \%$ 
- Containment radiation:

- Air particulate activity detector

- Gaseous activity detector

- Containment (high-range) area radiation monitor(s)

- Containment hydrogen concentration: $0.5 \%$ in dry air

- AC emergency buses: all energized

- AC service buses: all energized

- Emergency classification level: General Emergency

- Equipment inoperable

- All CFCUs

- Recirc-sump-to-RHR-suction isolation valves (EJ HIS-8811A and EJ HIS-8811B)

- Recirc-sump-to-containment-spray-suction isolation valves (EN HIS-1 and EN HIS-7)

- Procedure \& step in effect: ECA-1.1, step 11, try to start an RCP

- Assumptions: Earthquake greater than SSE; LOCA with loss of all AC power; loss of emergency coolant recirculation capability; recovery of two AC emergency buses; hydrogen burn in containment; containment spray actuation; loss of all containment fan cooler units

ERG network path:

ECA -0.0

ECA -0.2

E-1

ES-1.1

ES-1.3

ECA-1.1

\section{Connections to Decision-Making Model:}

\section{Monitor/ Detect:}

- Ability to differentiate between instrument failure and accident response: Do the panel members 'believe" the indications of severe core overheat-i.e., interpret the readings as indications of severe core overheat?

- Hot-leg temperature history indicates failed (open) RTDs

- CET temperature history indicates failed (open) thermocouples

\section{Interpret Current State:}

- Ability to develop complete and accurate mental representation of plant status: Do the panel members understand that some core melting and slumping probably occurred before SI was initiated?

Do the panel members consider the following current plant indications to assess the core damage state?:

- RCS hot-leg temperature pegged high and CET avg temperature at $0 \mathrm{~F}$ while SI has been in operation for over 1.5 hours and RVLIS static range is pegged high

- These indications are consistent with RTD and thermocouple failure (open circuits) in a previously hightemperature environment that has been mitigated subsequent to the instrument failure

- The containment hydrogen burn is indicative of severe core damage

Do the panel members try to determine the following?:

- Whether the progression of core damage has been arrested?

- Whether all of the core is in a coolable configuration?

- If the amount of melting and slumping that occurred prior to initiation of SI flow wasn't too extensive, the melted and slumped materials would probably re-freeze in a coolable configuration 
- The times given in the scenario are intended to be consistent with arresting of the melt/slump process just after the onset of that process

- Panel members cannot know the core damage state with certainty; they cannot be certain that severe accident progression has stopped

- Containment integrity should now be the highest priority because

- Many fission products were previously released to the containment

- Hydrogen burn did occur earlier

- Uncertain that severe accident has stopped

Do the panel members understand that the following potential challenges to containment integrity pose no immediate threat and are not likely to become serious threats any time soon?:

- High-pressure blowdown of RCS; RCS is already depressurized

- Hydrogen burn; hydrogen concentration has already been reduced to less than the burnable threshold by deflagration

Do the panel members understand that if the severe accident progression has not stopped, then the following longer-term threats to containment integrity could develop?:

- Molten corium, relocated to RPV lower head and shielded from SI flow by crusts, could attack RPV metal and cause vessel failure

- Corium pour could interact with water and/or concrete, increasing containment pressure over time

- Steam

- Hydrogen

- Non-condensibles such as $\mathrm{CO}$ and $\mathrm{CO} 2$

Determine Implications:

- Ability to remove bad or irrelevant ideas from consideration: Do the panel members consider the following?:

- Hydrogen burn may have damaged safeguards equipment inside of containment, e.g.,

- CFCUs

- Hydrogen recombiners

- Verifying that containment integrity is still intact following the hydrogen burn

- If so, how do they intend to do it?

- What parameters/ indications will they monitor to detect loss of containment integrity?

Do the panel members realize that containment spray (even with only a single spray pump) is a major demand on RWST inventory, and that the TSC engineer is absolutely correct when he says, "The spray pump is depleting RWST inventory at a much faster rate than we can ever hope to make up to it."

- There are two strategies or goals implicit in the TSC engineer's recommendation, viz.:

- Conserve RWST inventory by terminating spray and reducing the rate of depletion

- Find a way to make up to the RWST at a rate that approaches the reduced rate of depletion

Do the panel members try to devise either of the following?:

- A means of making up to the RWST at a rate in excess of $120-200 \mathrm{gpm}$

- Or, a means of supplying water to the suction of the ECCS pumps other than the RWST

Plan:

- Knowledge of criteria for applying HLA (conditions of applicability): Do the panel members perceive the following potential goal conflict?:

- Given the uncertainty that the severe accident progression has stopped and the possibility that the RPV might eventually fail, it might be advisable to leave the containment spray pump running (and even start the second spray pump)

- Significant amount of time and water are required to flood the reactor cavity

- If panel subsequently comes to consider RPV failure as likely, it would be beneficial to have containment already flooded to the point of filling the cavity

Do the panel members realize that the TSC engineer's recommendation is only a stop gap measure at best? 
- The core cooling safety function will eventually be challenged again, if:

- A means of making up to the RWST at a rate equal to the depletion rate is not found (the RWST will empty) or a suction supply to the ECCS pumps other than the RWST is not found AND

- ECCS recirculation capability is not restored

- Knowledge of limits of applicability for EOPs: If the panel members decide to stop all containment spray, how do they reconcile their decision with the procedural guidance of ECA-1.1?

- Knowledge of specific plant systems required for implementing plan or procedure: Do the panel members devise a feasible and effective means of either of the following?:

- Making up to the RWST at rate that approaches the depletion rate

- Supplying the suction of the ECCS pumps from a source other than the RWST 


\section{B.3 Glossary for PWR Scenarios}

$\begin{array}{ll}\text { AC } & \text { alternating current } \\ \text { AFW } & \text { auxiliary feedwater } \\ \text { ASAP } & \text { as soon as possible } \\ \text { BAST } & \text { boric acid storage tank } \\ \text { BATP } & \text { boric acid transfer pump } \\ \text { BIT } & \text { boron injection tank } \\ \text { CCP } & \text { centrifugal charging pump } \\ \text { CCW } & \text { core cooling water } \\ \text { CDA } & \text { core damage assessment } \\ \text { CET } & \text { core exit thermocouples } \\ \text { CFCU } & \text { containment fan cooler units } \\ \text { CO } & \text { carbon monoxide } \\ \text { CO2 } & \text { carbon dioxide } \\ \text { COP } & \text { cold over-pressurization } \\ \text { CSF } & \text { critical safety functions } \\ \text { DC } & \text { direct current } \\ \text { DCH } & \text { direct core heating } \\ \text { ECCS } & \text { emergency core cooling system } \\ \text { EDG } & \text { emergency diesel generator } \\ \text { EOF } & \text { emergency off-site facility } \\ \text { EOP } & \text { emergency operating procedures } \\ \text { ESW } & \text { essential service water } \\ \text { FRG } & \text { function restoration guidelines } \\ \text { FRP } & \text { function restoration procedures } \\ \text { H2 } & \text { hydrogen } \\ \text { HLA } & \text { high-level action } \\ \text { LOCA } & \text { loss of coolant accident } \\ \text { MOV } & \text { motor-operated valve } \\ \text { MWST } & \text { make-up water storage tank } \\ \text { OBE } & \text { operational-basis earthquake } \\ \text { PDP } & \text { positive displacement (charging) pump } \\ \text { PORV } & \text { power-operated relief valve } \\ \text { PWR } & \text { pressurized water reactor } \\ \text { PZR } & \text { pressurizer } \\ \text { RCS } & \text { reactor coolant system } \\ \text { RHR } & \text { residual heat removal } \\ \text { RPV } & \text { reactor pressure vessel } \\ \text { RTD } & \text { resistance temperature device } \\ \text { RVLIS } & \text { reactor vessel level indication system } \\ \text { RWST } & \text { reserve water storage tank } \\ \text { SA } & \text { severe accident } \\ \text { SAMG } & \text { severe accident management guidance } \\ \text { SG } & \text { steam generator } \\ \text { SGTR } & \text { steam generator tube rupture } \\ \text { SI } & \text { safety injection } \\ \text { SNUPPS } & \text { standard nuclear utility } \\ \text { SSE } & \text { safe-shutdown earthquake } \\ \text { technical support center } \\ \text { volume control tank }\end{array}$




\section{Appendix C}

\section{BWR Scenarios}

\section{Table of Contents}

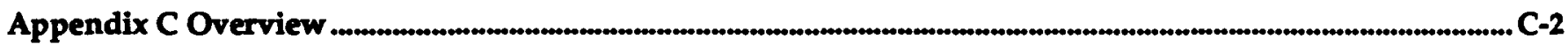

C.1 Scenario Summaries....1.n.

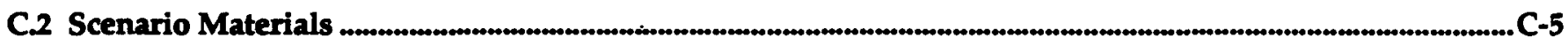

BWR-1: Potential Recriticality During Core Reflood.......................................................................................... C-5

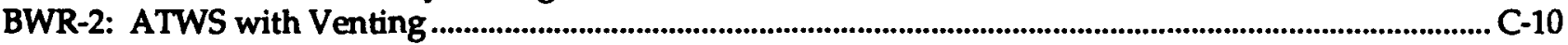

BWR-3: LOCA with Containment Flooding ….............................................................................................................. C-14

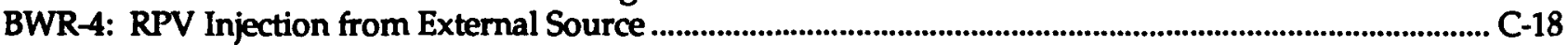

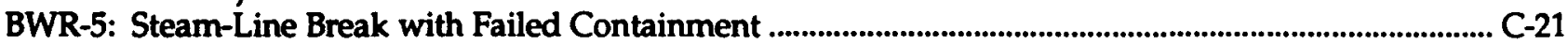

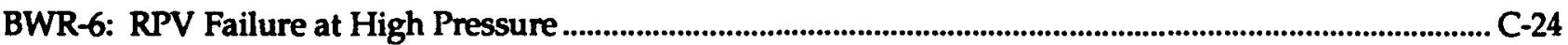

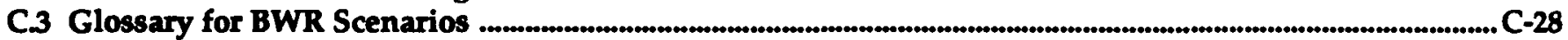




\section{Appendix C Overview}

This Appendix is split into two sections. The first section, Scenario Summaries, provides capsulized descriptions of each of the BWR scenarios. The second section, Scenario Materials, contains the detailed descriptions of each scenario and provides the detailed support material required to present each scenario to a panel (as described in Appendix A). A glossary of scenario acronyms can be found at the end of the Appendix.

\section{C.1 Scenario Summaries}

This section provides brief descriptions of the 6 BWR scenarios that were created for this project.

BWR-1: Potential Recriticality During Core Reflood. The purpose of this scenario is to create a core-melt situation in which the panel members must confront the implications of reflooding a damaged core, including the potential for recriticality.

In this scenario, a short-term station blackout leads to core uncovery and the early stages of core melt. Then sufficient emergency power is restored to allow reflooding of the RPV. When power is restored, core damage has progressed to the following stage: a significant number of control rod blades have melted and their neutron poison has relocated; a small percentage of the fuel rods have melted but the remaining fuel rods retain their original geometry. It is assumed that this core configuration will return to a critical condition for a period of time after reflood begins.

When the problem is turned over to the panel members, they must determine how and when to reflood the RPV and decide whether it is necessary to reflood the vessel with borated water in order to mitigate the recriticality threat during recovery. There is interest in whether the panel members realize there may be other ramifications from the reflood process than are normally expected. In addition to recriticality, these include the following: production of large quantities of steam and hydrogen; melting of fuel (caused by heat generated in the zirc-steam reaction); and shattering of the embrittled fuel cladding.
Panel members may have a perception of the situation that biases them to believe that after shutdown is achieved, it is maintained for all time, regardless of the course of events. Given the course of events and the possibility of bias, the panel may miss the recriticality issue and focus entirely on recovering the vessel inventory as quickly as possible by maximizing all available RPV injection.

Of interest is the panel's consideration and resolution of whether recriticality is an acceptable event, and how much of a reactivity response is expected. Also of interest is the panel's consideration of power control by boron injection, and whether the panel imposes limitations on the injection flow rate into the vessel. The HLAs/ strategies contained within the recommendation proposed in this scenario are to "Initiate SLCS" and to "Reflood the reactor vessel with borated water."

BWR-2: ATWS with Venting. The purpose of this scenario is to create a situation in which the panel members are forced to address two potentially conflicting goals, adequate core cooling (ACC) and containment integrity.

In this scenario, the MSIVs inadvertently close and an ATWS occurs. Alternate control rod insertion fails, both from the control room and from the hydraulic control units. Suppression-pool cooling is established but the SLCS fails to start. To control power, the operators reduce RPV water level to the top of the active fuel. They use the SRVs to reduce RPV pressure. Containment pressure approaches the primary containment pressure limit (PCPL). A decision on whether to vent containment is being held off because of the associated downside consequences and because of some progress in restoring SLCS.

The HLA/ strategy contained within the recommendation proposed in this scenario is to delay the venting of containment and to concentrate available resources on shutting down the reactor. This scenario attempts to focus the panel members on two goals. One is to shutdown the reactor while maintaining ACC. The other is to maintain containment integrity, which might require containment venting. However, venting can lead to loss of RPV injection (and loss of ACC), if the NPSH for $\mathrm{LPCI}$ becomes inadequate when the suppression pool flashes. Thus, the panel must confront and resolve a goal conflict. 
The panel has additional issues to resolve. Failure to achieve reactor shutdown is the source of the overpressure challenge to containment. The challenge can be completely removed only by shutting down the reactor. The vent ducting from the containment might fail into the reactor building, creating a harsh environment. Such an environment would hinder the ongoing repair efforts and might cause ECCS equipment subjected to it to fail. Furthermore, if the containment vent size is inadequate to remove sufficient energy, venting will buy some time but it will not remove the threat to containment and it could result in loss of $A C C$.

BWR-3: LOCA with Containment Flooding. The purpose of this scenario is to create a situation for which the EOPs direct that the containment be flooded to the top of the active fuel and then to probe the panel's understanding of the potential consequences of flooding by proposing an alternative to it.

In this scenario, an earthquake causes a large LOCA with a coincident loss of all $\mathrm{AC}$ power. Because of the depressurization and the unavailability of auxiliary steam, no turbine-driven systems were available for RPV injection. The core uncovered and had begun to heat up when on-site power was restored. Lowpressure injection into the RPV was established. However, because of the break size (DBA-type shear of reactor recirculation line), RPV water level cannot be raised to cover the top one third of the core. The fuel is intact, but many cladding ruptures have occurred, resulting in the release of a significant amount fission product gases to the containment.

The recommendation is made to flood the containment in accordance with the EOP (HLA/ strategy to "Flood dry-well containment following large LOCA"). Then, before flooding is commenced, an alternative is proposed in which concerns about flooding are raised. These concerns include the following: loss of pressure-suppression capability; loss of the wet-well vent after the torus fills, and loss of the capability to use the suppression pool for scrubbing. An additional concern is that flooding may make it necessary to vent the containment in order to prevent over-pressurization by noncondensable gases, including fission products. Early venting with minimum hold-up time will worsen the off-site dose.
As an alternative to flooding, the following plan is proposed: initiate LPCI in the dry-well spray mode in order to scrub airborne contaminants and condense steam; inject into the RPV with core spray systems; use the suppression pool as the source of all suction. The panel must weigh the benefits of this plan (maintenance of pressure suppression and wet-well venting capability and of suppression-pool scrubbing; prolonged hold-up of fission products in containment) against its main drawback: transport of contaminants through the ECCS piping. Additionally, the panel must decide whether to rely on the core spray system to cool the upper one third of the core.

Other issues that may complicate the decision-making process include the following. Pressure suppression and wet-well venting capability may be considered dispensable, provided that the reactor remains depressurized that containment cooling is maintained. Suppression pool scrubbing may be considered dispensable, if it is known the gaseous fission products in containment are only noble gases.

BWR-4: RPV Injection from External Source. The purpose of this scenario is to create a situation in which the panel members are forced to address two potentially conflicting goals, adequate core cooling (ACC) and containment integrity. The conflict is rooted in the effects of long-term injection into the RPV from an external source.

In this scenario, an earthquake causes an extended loss of all AC power. Injection into the RPV is maintained with steam-turbine-driven systems. A diesel-driven fire pump is lined up to take a suction on the CST and inject into the RPV, which is intentionally depressurized to establish injection flow. Water level in the RPV is maintained above the top of the active fuel. To conserve battery capacity, the steam-turbine-driven ECCS are secured. After several hours, suppression-pool water level rises to its high limit and containment pressure reaches the primary containment pressure limit.

The plant has reached a condition for which the EOPs direct that all injection from external sources be secured. The objective of this direction is to preserve containment integrity. A recommendation is made to deviate from the EOP by maintaining external injection. The HLAs/ strategies contained in the 
recommendation are to "Use diesel-driven fire pump for core injection" and "Use fire pump for vessel injection."

The line of reasoning offered in support of the recommendation is as follows: If external injection is secured, it will be necessary to resume injection from the steam-turbine-driven ECCS, with suction from the suppression pool. This will deplete battery capacity, which is needed to keep the SRVs open. Additionally, the high-temperature water from the suppression pool might cause the turbine-driven pumps to fail. Thus, action taken to preserve containment integrity could result in loss of ACC.

The existing EOPs try to accomplish several goals at once, but when a mutually exclusive decision must be made between ACC and containment integrity, containment integrity is given higher priority. The recommendation contains an argument against this. The challenge to containment posed by a core that goes ex-vessel following loss of ACC is quite severe. The potential for serious off-site dose is much greater than for the case in which containment fails but ACC is maintained and the core never melts.

BWR-5: Steam-line Break with Failed Containment. The purposes of this scenario are to create a situation in which both the RPV and the containment are breached but the core is intact and seems likely to remain so. The panel is asked to devise ways to minimize fission product leakage from the containment, mitigate the harsh environment in the reactor building, and maintain ACC.

In this scenario, an unisolable, DBA-type steam-line break leads to some cladding failure and fission gas release. The containment is isolated, except for a failed electrical penetration seal in the dry-well, which allows leakage into the reactor building. Lowpressure ECCS systems restore water level in the depressurized RPV to normal.

The panel must attempt to configure the available systems in such a way as to minimize flow into the reactor building. The harsh environment created in the reactor building must be mitigated not only to allow access for repairs, but also to ensure that necessary equipment is not degraded. The only real threat to $A C C$ is the potential loss of equipment that is not qualified for the harsh environment in the reactor building. The partial failure of containment makes the maintenance of $A C C$ even more important.

BWR-6: RPV Failure at High Pressure. The purpose of this scenario is to create a situation in which the core melts while the RPV remains at high pressure: With RPV failure imminent, the panel is asked to decide how to protect containment. The panel is confronted with a variety of post-vessel failure issues.

In this scenario, inadvertent MSIV closure causes the plant to trip. Feedwater is lost and all high-pressure injection fails. Low-pressure injection is available but the RPV cannot be depressurized. The SRVs, ADS, and all other methods of depressurization have failed. RPV inventory boils off and the core uncovers.

Extensive fuel melting occurs.

Suppression-pool cooling is secured so as not to circulate contaminants outside of containment. Because RPV water level is unknown, flooding of containment is commenced. Then, after wet-well water level rises above its high limit, the decision to flood is countermanded, because the RPV is still at pressure.

The panel must decide how to protect containment. The recommendation proposes that dry-well spray be initiated. The HLA / strategy is to "Initiate dry-well spray to mitigate HPME." Questions are posed about high-pressure melt ejection, the effect of water on the blowdown, and the potential for direct containment heating. The panel is asked whether it would be better to have water in the containment before RPV failure or to postpone flooding until after failure. 


\section{C.2 Scenario Materials}

BWR-1: Potential Recriticality During Core Reflood

Warning:

This scenario is intended to be used as a tool or exercise for generating a response from a small accidentmanagement team (or panel) to a hypothetical severe accident. The function of this accident scenario is to present a situation that pushes the team outside of the standard, well-rehearsed procedures for addressing abnormal plant conditions. To achieve this, we created scenarios with difficult choices, goal conflicts, or unique situations that present significant challenges to the team's decision-making process.

Further, to create the necessary severe accident conditions in this scenario, it was necessary to postulate numerous traumas, equipment failures, and at times, operator errors. The use of multiple failures was required to thwart the safety and defense-in-depth design employed in nuclear power plants and reduce the effectiveness of current emergency operating procedures (EOPs).

The boundary conditions of the scenario were constructed for the sole purpose of maintaining a difficult decisionmaking situation for as long as possible. Therefore, it should not be construed that these scenarios are likely events, or even the most likely severe accident sequences. The probability of these scenarios occurring is within, or beyond, the range of traditional IPE core-damage frequencies.

\section{Scenario Objective:}

The objectives of this scenario are as follows:

- To place the panel members in a core-melt situation that may challenge the emergency operating procedures (EOPs).

- To determine if the panel recognizes the potential threat of recriticality along with other challenges associated with vessel reflood.

- To present technical problems that will challenge the panel to decide whether to reflood immediately or wait until the necessary amount of boron has been injected into the reactor pressure vessel (RPV) to ensure shutdown.

\section{Scenario Assumptions:}

These assumptions are for the presenter and are not to be presented to the panel:

- During a surveillance, the reactor core isolation cooling (RCIC) [or equivalent system] was found to be inoperative. RCIC [or equivalent] system was declared inoperative and disabled.

- The plant is operating at normal full power conditions.

\section{Progression of Events:}

$0 \mathrm{~min}$. Loss of all off-site power initiates a turbine trip, reactor scram, MSIV isolation, and a feedwater trip. All of the plant's emergency diesel generators fail to start.

$1 \mathrm{~min}$. Operators attempt to manually initiate HPCI; HPCI turbine steam injection valve 
fails to open [omit if ECCS HP motor-driven system]. Classic short-term IPE station black-out (SBO) scenario.

RPV inventory decreases by boil-off to the suppression pool via the safety relief valves. Auxiliary operators and maintenance personnel dispatched to access and repair HPCI [omit if ECCS HP motordriven system], and emergency diesel generators. Operators declare site area emergency. Activation of the TSC and the EOF begins.

$60 \mathrm{~min}$. RPV water level drops to the top of active fuel (TAF), operators declare general area emergency.

95 min RPV water level drops below Minimum Steam Cooling Level, operators perform emergency RPV depressurization per EOPs.

$122 \mathrm{~min}$ Control rod blades begin melting. Blade temperature approximately $2600 \mathrm{~F}$.

$125 \mathrm{~min}$ Fuel cladding begins melting. Fuel temperature approximately $3365 \mathrm{~F}$.

$132 \mathrm{~min}$ Fuel begins melting. Fuel temperature approximately $4870 \mathrm{~F}$.

133 min Significant number of the control rod blades have melted and relocated, while a small percentage of the fuel rods have melted. The remaining fuel rods are assumed to be in their original geometry. Emergency diesel generator B is repaired and available to restore AC power to an ECCS motor control center (MCC). RPV injection is, therefore, available with only one train of low pressure coolant injection (LPCI) and core spray (CS) systems.

\section{Other Notes:}

- The core configuration, as described above, is assumed to be sufficient to achieve criticality for a period of time after core reflood begins. Furthermore, only two ECCS systems are available for vessel injection-namely, $\mathrm{LPCI}$ and CS. These are the present boundary conditions available to the accident management team.

\section{Statement for Panel:}

The plant had been operating at $100 \%$ power for greater than 200 days when, during a normal surveillance, RCIC [or equivalent] system was determined to be inoperative and was secured. Several hours later, a large storm disabled the power transmission grid, which caused the plant to lose all off-site power. This event initiated a turbine trip, reactor scram, and MSIV isolation. After the reactor scram, an operator verified that all the control rods were fully inserted by the rod-position indication system. During these initial events, the operators quickly determined that all of the emergency diesel generators had failed to start and would not respond to manual initiation. In anticipation of the need for level control, operators attempted to start the HPCI system that failed to start upon manual and automatic initiation [omit if ECCS HP motor-driven system].

Following these events, the control room supervisor dispatched auxiliary operators and maintenance crews to diagnose and repair, if possible, the failed HPCI [omit if ECCS HP motor-driven system] and the emergency diesel generators. A site emergency was declared and the TSC and EOF were activated. It was determined that the HPCI turbine steam injection valve had seized in the closed position and could not be immediately repaired [omit if ECCS HP motor-driven system].

While the maintenance crew was assessing the problem with the generators, the RPV water level continued to decrease. Level instrumentation indicated that the top of active fuel was uncovered approximately one hour into the event. At this time, a general area emergency was declared. Level instrumentation indicated that the core 
water level decreased below the EOP limit for RPV steam cooling at an hour and a half into the event. The operators commenced with emergency RPV depressurization per the plant EOPs. Approximately thirty minutes after the depressurization, hydrogen and high radiation were detected in the containment.

It is now 133 minutes since the plant lost off-site power and one diesel generator has been repaired and is available.

\section{Recommendation:}

At this time an engineer in the TSC makes the following recommendations:

"From our containment instrumentation, we know that some fuel damage has occurred due to the amount of hydrogen and high radiation detected. I believe that most of the fuel geometry is still intact, but we need to act quickly to prevent further damage such as fuel relocating into the lower plenum. Such a situation would present an extreme challenge to the lower RPV head and may induce vessel failure. Therefore, I suggest we establish RPV injection systems and flood-up the vessel until level instrumentation indicates the water level is above the top of active fuel."

"Since we know that hydrogen generation from oxidation is driven primarily by the temperature of cladding and the availability of steam-of which there will be plenty when we reflood-the quicker we cool the fuel, the less overall hydrogen and heat we will generate. Based on this assumption I recommend maximizing the injection of the LPCI and CS system during the vessel reflood. If we reflood in this manner, we may be able to reduce the total amount of hydrogen generated and minimize further damage to the reactor core."

\section{Initial Questions to Panel:}

- Should this recommendation be implemented? Why or Why not?

- Are the hazards associated with the engineer's concerns valid?

- Have all the potential hazards associated with the vessel reflood been identified?

\section{Follow-up or Probing Questions:}

At this time, DG B is started and power to the CS and LPCI system bus is re-established, but the pumps have not been started. A reactor engineer from the TSC/OSC brings up the following issue:

"From reports and analysis I have read about BWR severe accidents I know there is a time window during which the control rods, which are made out of boron carbide cladded in stainless steel, can start to melt and relocate, yet the fuel rods themselves can still be essentially in their original geometry. This is because the eutectic melting point of the control blade is around $2300 \mathrm{~F}$ to $2500 \mathrm{~F}$ while the melting point of the fuel rods is much higher (approximately 4500 F)."

- What are your recommendations for reflood given the engineer's information?

- What would be your recommendations given the following additional information from the engineer:

"My concern is that if a significant number of control rods have melted and relocated and we re-establish injection, we might drive the reactor super critical, thereby further damaging the fuel and maybe even the vessel. Therefore, I would recommend that we first inject the required shutdown weight of boron via the SLC system before we initiate the core reflood to ensure the reactor will stay subcritical."

- How can boration be added to the vessel?

- What if standby liquid control (SLC) system is unavailable?

- Is there enough boration on site to borate the suppression pool to the proper level required $(700 \mathrm{ppm})$ ?

- Are there concerns regarding using core spray (CS) to reflood the core in an ATWS- like event? 
- What other precaution can be implemented to protect the vessel and containment?

- Will the reactor maintain criticality after reflood or will the fuel shatter and lose the necessary geometry for criticality

- If a debris bed forms from the postulated fuel shattering, will this geometry be more difficult to cool?

- Are the engineer's concerns regarding supercriticality valid?

- Is the time window for recriticality large?

\section{Background Material for Conducting Exercise:}

\section{Current Plant Conditions}

- RPV Pressure: 10 psig

- RPV Level: downscale

- RPV Power: downscale

- DW Pressure: 3 psig

- DW Temp: $140 \mathrm{~F}$

- SP Temp: $175 \mathrm{~F}$

- H2 Conc: $>6 \%$

- O2 Conc: downscale

- Essential DC: available

- Off-site AC: unavailable

- Diesel Gen: DG B only available

- ECCS: turbine-driven: unavailable motor-driven: 1 train of LPCI \& CS only

- Cont Rad: High radiation detected

- PASS system: Xe, Kr, CsI, CsOH (if available in SBO condition)

- Assumptions: Short-term SBO scenario at the point of early core melt. A significant amount of boron from the control rod blades have relocated during the accident, while most of the fuel rods are still in their original positions, i.e., potential for recriticality and return to power exists during vessel reflood.

\section{Connections to Decision-Making Model:}

\section{Interpret Plant State:}

- Knowledge of plant-specific condition and system availability: After presentation of the scenario background, do the panel members realize that:

- the plant has experienced an SBO

- one (1) DG is available for 4.16 KVA AC to the MCC

- one train of CS and LPCI are available for core reflood

\section{Determine Implications:}

- Knowledge of critical physical phenomena during severe accident progression: Do the panel members recognize that:

- the control rods may have been damaged

- the core conditions are such that the fuel temperature is elevated and the core is "steam-starved"

- Ability to anticipate the phenomenological consequences of action/or inaction: Do the panel members recognize that:

- if the control rods are damaged, the potential for recriticality exists during reflood

- a large amount of steam will be created during the reflood

- the steam created during reflood will feed the clad-oxidation process, thus creating hydrogen 
- the time window for recriticality may only be a short one

- the fuel may shatter during the reflood

Plan:

- Knowledge of criteria for applying HLA (condition of applicability): Do the panel members know:

- a goal conflict exists between immediate ACC and avoiding recriticality

- that recriticality may be acceptable

- how to minimize the reactivity insertion consequences

- that SLC injection will take time to establish the necessary hot shutdown weight of Boron

- Knowledge of specific plant systems: Can the panel devise a method to inject borated water during reflood if SLC is unavailable?

- Knowledge of emergency procedures and their functions.

- if the panel identifies that the reactor may go recritical, will the panel inject boron prior to, or at the same time during reflood?

- based on EOP level/power control, will the panel use CS during reflood?

- Knowledge of criteria for applying high-level action. Will the panel:

- decide that the recriticality is of lower importance than core cooling?

- know the "worst-case" effect of the reactivity insertion?

\section{Execute:}

- Ability to prioritize action when implementing resources. Does the panel:

- recognize that time is of the essence, and finding a method to borate before (if SLC is unavailable) will make the accident worse.

- decide to reflood first and borate later, if possible

- Knowledge of severe accident events and their symptoms. Do the panel members recognize that:

- the core is degraded

- the potential of fuel and control rod melting has occurred

- Ability to identify specific marker/cues given a diagnosis. Do the panel members recognize that:

- fission products have been detected in the containment, i.e., fuel damage has occurred

- hydrogen has been detected in the containment, i.e., fuel damage has occurred 


\section{BWR-2: ATWS with Venting}

\section{Warning:}

This scenario is intended to be used as a tool or exercise for generating a response from a small accidentmanagement team (or panel) to a hypothetical severe accident. The function of this accident scenario is to present a situation that pushes the team outside of the standard, well-rehearsed procedures for addressing abnormal plant conditions. To achieve this, we created scenarios with difficult choices, goal conflicts, or unique situations that present significant challenges to the team's decision-making process.

Further, to create the necessary severe accident conditions in this scenario, it was necessary to postulate numerous traumas, equipment failures, and at times, operator errors. The use of multiple failures was required to thwart the safety and defense-in-depth design employed in nuclear power plants and reduce the effectiveness of current emergency operating procedures (EOPs).

The boundary conditions of the scenario were constructed for the sole purpose of maintaining a difficult decisionmaking situation for as long as possible. Therefore, it should not be construed that these scenarios are likely events, or even the most likely severe accident sequences. The probability of these scenarios occurring is within, or beyond, the range of traditional IPE core-damage frequencies.

\section{Scenario Objective:}

The objectives of this scenario are as follows:

- To place the panel members in a goal-conflict situation that involves venting containment and providing adequate core cooling.

- To determine if the panel recognizes the potential loss of adequate core cooling as a possible consequence of containment venting.

- To present technical problems that challenge the panel to decide whether to protect the containment integrity or maintain adequate core cooling.

\section{Scenario Assumptions:}

These assumptions are for the presenter and are not to be presented to the panel:

- The plant is operating at normal full-power conditions.

- The reactor has experienced an ATWS event, SLC is inoperative, and the containment is being challenged by over-pressurization via suppression-pool boiling.

- Plant vent pipes sized large enough for ATWS power ( $>5 \%$ of full power) are assumed to be duct work inside the reactor building. This assumption is not valid for all plants.

\section{Progression of Events}

0 min. Inadvertent MSIV isolation causes a turbine trip and feedwater trip, but the reactor fails to scram.

$1 \mathrm{~min}$ Operators quickly identify the anticipated transient without scram (ATWS) condition and enter the plant's EOPs. Alternate means of control rod insertion from the control room fails. Operators initiate level control with the HPCI system [or ECCS HP alternate]. 
2 min Operators begin suppression-pool cooling because of elevated pool temperatures.

When the boron injection initiation temperature (BIT) is reached (110 F) operators attempt to initiate standby liquid control (SLC) system, which fails to start. Operators continue to maintain RPV pressure control and commence to lower RPV level to TAF for power/level control.

$5 \mathrm{~min}$ Operators open cycling SRVs to reduce RPV pressure.

15 min Alternate means of inserting control rods and injecting boron are unsuccessful.

Operators secure low pressure injection systems and HPCI [or ECCS HP alternate and depressurize RPV because of heat capacity temperature limit (HCTL).

$100 \mathrm{~min}$ Containment pressure approaches primary containment pressure limit (PCPL) (60 psig).

\section{Statement for Panel:}

Approximately an hour and forty minutes ago, the plant experienced an inadvertent MSIV isolation that caused a feedwater and turbine trip. Operators determined that the reactor failed to scrarn and entered the plant's EOPs. Alternate methods of control rod insertion from the control room were not successful. When the BIIT was reached, the operators attempted to initiate standby liquid control (SLC) system, which failed to respond. While auxiliary operators were dispatched to determine the cause of this problem, other personnel were attempting to insert control rods locally at the hydraulic control units (HCU) of the control rod drives (CRD) without success.

The control room operators controlled reactor power and RPV pressure and level per the EOPs. Operators opened cycling SRVs to maintain reactor pressure and commenced lowering water level to the top of active fuel (TAF). RPV pressure was reduced to maintain RPV pressure below the heat-capacity temperature limit (HCTL) curve. During these operations, suppression-pool cooling was established. Attempts to re-open the MSIVs and re-establish the main condenser as a heat sink were unsuccessful. The operators were attempting to keep RPV water level near TAF by throttling the LPCI injection valve, but oscillations in the reactor pressure and power were making that task difficult. The drywell and wetwell sprays have not been used.

The decision to vent has been delayed because progress is being made on restoring the SLC system to operation.

\section{Recommendation:}

At this time an engineer in the TSC makes the following recommendations:

"We are rapidly approaching the primary containment pressure limit (PCPL). We have postponed performing containment venting because of two possible major problems we may encounter if we do vent:

First, we may lose our NPSH requirements for the LPCI injection system when the suppression pool flashes during the venting procedure. This may lead to a core melt situation if we do not have means to re-establish RPV injection.

Second, the vent pipes from the containment feed into duct work located in the reactor building, which will fail when we vent. This would render certain parts of the reactor building inaccessible because of the harsh environment. If this happens, it may preclude some or all of our repair efforts and quite possibly affect the injection systems we are counting on to maintain core cooling.

I believe we should postpone venting for as long as possible and concentrate our efforts on shutting down the reactor either by control rod insertion or alternate boron injection." 


\section{Initial Questions to Panel:}

- Should this recommendation be implemented? Why or why not?

- Assuming that venting would result in the loss of core cooling, would you vent the containment?

- Is established adequate core cooling with a failed containment in this situation acceptable?

- Have all the potential hazards been identified?

- What would your decision be if you knew that the wetwell vent is inoperative?

- What affect would WW or DW sprays have on the accident progression?

\section{Background Material for Conducting Exercise:}

\section{Current Plant Conditions}

- RPV Pressure: 100 - 300 psig oscillating

- RPV Level : oscillating around TAF

- RPV Power : oscillating 5-17\%

- DW Pressure : 60 psig

- DW Temp: $308 \mathrm{~F}$

- SP Temp: $308 \mathrm{~F}$

- H2 conc: downscale

- O2 conc: downscale

- DC Power : available

- AC power: available

- ECCS: available

- Cont Rad: normal

- Assumption: ATWS scenario, control rods cannot be inserted and SLC is not available. The containment is challenged by over-pressurization via suppression-pool evaporation. Containment venting presented as a potential challenge to reactor building equipment needed to support adequate core cooling functions.

\section{Connections to Decision-Making Model:}

\section{Interpret Plant State:}

- Knowledge of plant-specific condition and system availability: After presentation of the scenario background, does the panel realize that:

- plant has experienced an ATWS event

- ATWS power is controlled by pressure and level

- containment challenge is from the inability to shut down the reactor (via SP boiling)

\section{Determine Implications:}

- Knowledge of accident progression: Does the panel realize that

- containment challenge from over-pressurization is due to reactor power

- containment venting may not alleviate the problem

Plan:

- Does the panel realize that:

- one goal is to shut down the reactor while maintaining ACC, via boration, control rod insertion, and level/pressure control 
- another goal is to maintain containment integrity by power control, containment heat removal, and venting

- a goal conflict may ensue in which one goal may have to be prioritized over the other

- Do the panel members realize that a goal conflict may ensue if venting is performed.

- Knowledge of EOP/SAMG: Does the panel know the applicable EOPs for this situation.

- Ability to mentally simulate progression of the potential consequence of failure if the vent pipe fails in reactor building and ACC is lost.

- Knowledge of specific plant systems required for implementing plan or procedure. Does the panel know:

- ways to borate injection sources

- the time window to implement the vent

- the necessary valve alignment to establish the vent 


\section{BWR-3: LOCA with Containment Flooding}

\section{Warning:}

This scenario is intended to be used as a tool or exercise for generating a response from a small accidentmanagement team (or panel) to a hypothetical severe accident. The function of this.accident scenario is to present a situation that pushes the team outside of the standard, well-rehearsed procedures for addressing abnormal

plant conditions. To achieve this, we created scenarios with difficult choices, goal conflicts, or unique situations that present significant challenges to the team's decision-making process.

Further, to create the necessary severe accident conditions in this scenario, it was necessary to postulate numerous traumas, equipment failures, and at times, operator errors. The use of multiple failures was required to thwart the safety and defense-in-depth design employed in nuclear power plants and reduce the effectiveness of current emergency operating procedures (EOPs).

The boundary conditions of the scenario were constructed for the sole purpose of maintaining a difficult decisionmaking situation for as long as possible. Therefore, it should not be construed that these scenarios are likely events, or even the most likely severe accident sequences. The probability of these scenarios occurring is within, or beyond, the range of traditional IPE core-damage frequencies.

\section{Scenario Objective:}

The objectives of this scenario are as follows:

- To place the panel members in a recovery situation that may challenge the existing EOPs.

- To determine if the panel recognizes the implications of containment flooding after core damage (i.e., possible early drywell venting of noble gases).

- To present technical problems that challenge the panel to decide whether to establish long-term adequate core cooling recovery with core spray in a recirculation mode, or to flood-up the containment.

\section{Scenario Assumptions:}

These assumptions are for the presenter and are not to be presented to the panel:

- The plant is operating at normal full power conditions.

- RPV water level cannot be restored above the top of active fuel because of a large non-isolated breach in the reactor recirculation piping. The fuel is intact, but a large number of clad ruptures have occurred that released a significant amount of gap fission product gases to the containment.

\section{Progression of Events:}

0 min Occurrence of a large earthquake causes the loss of normal and emergency AC power with the exception of essential $A C$ supplied via the $D C$ invertors (all diesel generators fail to start). Furthermore, the earthquake causes a shear of reactor recirculation line (DBA-type accident).

The core uncovers and begins to heat up. Operators declare general area emergency. Activation of the TSC and EOF begins.

$35 \mathrm{~min}$ On-site power is recovered. Operators commence RPV injection with low pressure ECCS systems. Significant amount of hydrogen has been generated; level cannot be recovered above top of active fuel. 


\section{Statement for Panel:}

Thirty five minutes ago a large earthquake occurred causing the plant to experience a loss of all off-site power. All emergency diesel generators failed to start, rendering the plant in a situation where all normal and emergency $A C$ was lost with the exception of the essential AC supplied via the DC invertors. During the event, it was observed that the reactor experienced a rapid depressurization and the containment pressure and suppressionpool temperature increased quickly. It was assumed that the plant experienced a large-LOCA-type event. Operators confirmed that the reactor scrammed and that the containment isolated. Because of the depressurization and the unavailability of auxiliary steam, no turbine-driven systems were available for RPV injection.

Five minutes ago the on-site $A C$ power was restored via the diesel generators. RPV injection was established via the motor-driven systems. During the attempted re-flood of the vessel, containment instrumentation indicated the presence of hydrogen and radiation. The operators have been unsuccessful at raising the water level above the top of the active fuel.

\section{Recommendation:}

At this time an engineer in the TSC brings up the following issues:

"It appears that the plant has experienced a large DBA-type LOCA accident induced by the earthquake such that we cannot re-establish the RPV water level above the top of active fuel. Our PASS system indicates $\mathrm{Xe}$ and $\mathrm{Kr}$ in the containment, but no significant amount of Cs or I. I believe that we have experienced numerous clad failures of our fuel rods, but little if any fuel pin damage.

We have not been able to raise the water level above the top of active fuel. In this case we are directed per EOPs to flood the containment to the top of active fuel to ensure we have established adequate long-term core cooling. This flooding may also provide additional benefits such as protecting the bottom of the RPV, in case any fuel has melted and relocated to the lower plenum, which I doubt has happened."

\section{Initial Questions to Panel:}

- What are your recommendations for containment flooding?

- Can adequate core cooling be assured with core spray injection in this configuration?

- What are the fundamental issues associated with containment integrity, adequate core cooling, and minimal radioactive release in this situation?

\section{Follow-up and Probing Questions:}

At this time the engineer in the TSC brings up the following issues:

"I have some major concerns regarding containment flooding that I think need to be addressed. First, if we flood the containment, we will lose our pressure suppression capability when the suppression-pool level covers the wetwell to drywell vacuum breakers. Second, we will lose our capability to vent through the wetwell, which will remove our capability of using the suppression pool as an effective scrubbing mechanism. Last, when we start to flood up, we will develop a non-condensible bubble in the upper portion of the drywell consisting of nitrogen, hydrogen, and certain fission products such as xenon and krypton. As we continue to flood, this gas will start to pressurize because of gas space reduction. This situation may force us to vent the containment via the drywell to protect against over-pressurization. Venting the fission product gases so early in the accident without maximizing the hold-up time may have a major radiological impact in terms of off-site dose. 
I would like to suggest the following alternate strategy. First, we place one train of LPCI in drywell spray mode to scrub any airborne contaminants and reduce the amount of steam in the drywell. Second, we continue to inject into the vessel especially with core spray systems using the suppression pool as the source of all suction. The drawback of this approach is that we are transporting contaminants through the ECCS piping. The positive side of this is that there will be water on the floor to help protect the containment if the lower head fails, we still have the capability to vent in wetwell, and we maintain our suppression capability.

Also, even though we can only maintain the core $2 / 3$ covered, the upper $1 / 3$ can be cooled via the core spray system, which has been verified in our FSAR analysis. If we can maintain containment heat removal via the RHR system, we can maximize the hold-up time of fission products before we have to vent the containment."

- Now, what are your recommendations for containment flooding?

- Can adequate core cooling be assured with core spray injection in this configuration?

- What are the fundamental issues associated with containment integrity, adequate core cooling and minimal radioactive release in this situation?

\section{Background Material for Conducting Exercise:}

\section{Current Plant Conditions:}

- RPV Pressure: 20 psig

- RPV Level : $2 / 3$ core height

- RPV Power : downscale

- DW Temp: $160 \mathrm{~F}$

- DW Pressure : 20 psig

- SP Temp: $190 \mathrm{~F}$

- H2 Conc: 5\%

- O2 Conc: downscale

- Essential DC: available

- Off-site AC: unavailable

- On-site AC: available

- ECCS: turbine-driven: unavailable; motor-driven: available

- PASS indicates Xe, $\mathrm{Kr}$ in containment

- Assumption: The plant has experienced a DBA-like LOCA that cannot be isolated in conjunction with a loss of AC power. After power is recovered, it is determined that the core cannot be flooded above the top of active fuel because of the break size.

\section{Connections to Decision-Making Model:}

\section{Interpret Current State:}

- Ability to develop complete and accurate mental representation: Do the panel members recognize:

- that the level cannot be restored above TAF

- that RPV injection is spilling from the break to the containment

- the submerged part of the core is adequately cooled, while the upper portion of the core is covered by steam and CS flow

- whether CS is considered adequate for cooling in this configuration

- that CS flow pattern is an important consideration 


\section{Determine Implications:}

- Ability to anticipate the consequences of an action or initiation. Do the panel members realize that:

- if core spray is not sufficient to cool the core (EOP position vs. FSAR), that the containment must be flooded

- by flooding, a bubble is squeezed in the containment airspace

- by venting to relieve the bubble, noble gases are released

\section{Plan:}

- Knowledge of EOPs. Do the panel members know that per EOPs, containment flooding is required?

- Knowledge of specific plant system required. Do the panel members know:

- the methodology for containment flooding

- the methodology for containment venting in the drywell 
BWR-4: RPV Injection from External Source

\section{Warning:}

This scenario is intended to be used as a tool or exercise for generating a response from a small accidentmanagement team (or panel) to a hypothetical severe accident. The function of this accident scenario is to present a situation that pushes the team outside of the standard, well-rehearsed procedures for addressing abnormal plant conditions. To achieve this, we created scenarios with difficult choices, goal conflicts, or unique situations that present significant challenges to the team's decision-making process.

Further, to create the necessary severe accident conditions in this scenario, it was necessary to postulate numerous traumas, equipment failures, and at times, operator errors. The use of multiple failures was required to thwart the safety and defense-in-depth design employed in nuclear power plants and reduce the effectiveness of current emergency operating procedures (EOPs).

The boundary conditions of the scenario were constructed for the sole purpose of maintaining a difficult decisionmaking situation for as long as possible. Therefore, it should not be construed that these scenarios are likely events, or even the most likely severe accident sequences. The probability of these scenarios occurring is within, or beyond, the range of traditional IPE core-damage frequencies.

\section{Scenario Objective:}

The objectives of this scenario are as follows:

- To place the panel members in a potential core-melt situation for which the emergency operating procedures may be challenged.

- To force the panel members to decide whether to preserve adequate core cooling or containment integrity in a situation where it may not be possible to achieve both.

\section{Scenario Assumptions:}

These assumptions are for the presenter and are not to be presented to the panel:

- The plant is operating at normal full power conditions.

\section{Progression of Events:}

$0 \mathrm{~min}$ Large earthquake causes the loss of normal off-site power. On-site emergency diesel generators fail to start. The plant experiences an MSIV isolation and a reactor scram.

$1 \mathrm{~min}$ Operators initiate steam-driven turbine injection systems to maintain reactor pressure vessel (RPV) level.

240 min Operators line-up a HPSW injection system with power supplied by a portable diesel generator. Water is supplied to the system from an external water source. Operators depressurize the RPV via SRVs and secure turbine-driven systems. HPSW injection established into the vessel for level control.

$960 \mathrm{~min}$ The suppression pool reaches saturation conditions. 
$1200 \mathrm{~min}$ Suppression-pool level above high limiting condition of operation (LCO)

level; containment pressure approaching 60 psig while containment water level above MPCWLL.

\section{Statement for Panel:}

Approximately twenty hours ago the plant experienced a large earthquake that caused the loss of all off-site and on-site $A C$ power, except the AC power supplied by the DC invertors. While repairs were underway, injection to the RPV was maintained with steam-driven turbine systems. The plant staff was successful in lining up and establishing an injection path to the vessel with a diesel-driven fire pump [or a city fire truck, or a pump from unit 2] with suction from the CST [or some other external water source]. Operators successfully depressurized the RPV and established injection into the vessel. The flow rate of this configuration is just enough to maintain the level in the RPV at about a foot above the top of active fuel. This was established at four hours into the event.

Operators have attempted to back up the battery capacity of the SRV solenoids and have conserved use of the batteries as much as possible. The steam-driven turbine systems were secured for this purpose. The suppressionpool temperature reached saturation at approximately 16 hours into the event.

Over the course of this accident the suppression pool water level has risen slowly. The suppression-pool water level has now reached the high-LCO-level mark and the containment is at approximately 60 psig.

\section{Recommendation:}

At this time in the event, an engineer in the TSC makes the following recommendations:

"We need to make a decision. We have reached the point at which the MPCWLL will be exceeded because of the water being added to the vessel from external sources. We are directed by the EOPs at this time to secure all injection from external sources regardless of adequate core cooling considerations. This step is prescribed in order to maintain the integrity of the containment. But, if we comply with this step and we are not successful at establishing core cooling from the suppression pool, we will most certainly proceed into a core melt situation. There are two consequences that need to be considered if we secure our diesel pump and use our steam-driven turbine systems with suction from the suppression pool. First, the turbine use will increase the drain on the remaining batteries, which we want to conserve in order to keep the SRVs open. Second, the suppression-pool temperature is high enough that turbine-driven pumps may fail and we would be without core cooling. Therefore, I believe we should maintain the present injection configuration. If we melt the fuel and the vessel fails I am not convinced that the containment integrity would be preserved. But if we can prevent the fuel from overheating in the first place, then the off-site consequences will surely be minimized. If the containment fails, so be it. A configuration of adequate vessel injection and a failed containment is acceptable to me versus all the potential problems associated with molten core material on the floor of the containment."

\section{Initial Questions to Panel}

- What are your recommendations for this situation?

- Given an ideal case of adequate core cooling (ACC) versus containment integrity, which would you choose, and why?

- What assurances would you need that ACC can be maintained, and is a given amount of time part of the answer?

- What would you do if the SRVs lost all power and the reactor begins to repressurize? 


\section{Background Material for Conducting Exercise:}

\section{Current Plant Conditions:}

- RPV Pressure: 60 psig

- RPV Level: TAF

- RPV Power: downscale

- DW Temp: $308 \mathrm{~F}$

- DW Pressure: 60 psig

- SP Temp: $308 \mathrm{~F}$

- H2 conc: downscale

- O2 conc: downscale

- Essential DC : available

- Off-site AC: unavailable

- ECCS: turbine-driven: secured; motor-driven: unavailable except for a diesel-driven fire pump

- Assumption: SBO scenario, RPV depressurized, injection available in diesel-driven fire pump. High containment pressure and high suppression pool LCO limit.

\section{Connections to Decision-Making Model:}

\section{Interpret Current State:}

- Knowledge of plant-specific conditions and system availability: After presentation of the scenario background, do the panel members realize that:

- the plant has experienced an SBO

- injection is established via a diesel-driven fire pump

- injection source is external to the containment

\section{Determine Implications:}

- Knowledge of critical physical phenomena during severe accident progression: Do the panel members recognize that:

- fuel debris in the containment will provide a significant challenge to containment integrity.

- associated challenges are liner attack, FCI, and CCI

\section{Plan:}

- Knowledge of criteria for applying HLA (condition of applicability): Do the panel members know:

- a goal conflict exists between ACC and containment integrity

- securing external injection may lead to fuel melt

- fuel melt may provide a significant challenge to the containment

- Knowledge of specific plant systems: Can the panel devise a way to lower the S/P level.

- Ability to prioritize action when implementing resources: Will the panel:

- decide to maintain ACC

- give up ACC for containment integrity

- Knowledge of severe accident events and their symptoms: Do the panel members recognize that if ACC is not established, fuel melt and vessel failure will occur?

- Knowledge of EOPs and their function: Does the panel identify that the EOPs require termination of outside containment injection source.

- Knowledge of criteria for applying high-level action: Will the panel decide to:

- risk ACC by securing the external injection

- decide to maintain ACC at the price of containment integrity 
BWR-5: Steam-Line Break with Failed Containment

\section{Warning:}

This scenario is intended to be used as a tool or exercise for generating a response from a small accidentmanagement team (or panel) to a hypothetical severe accident. The function of this accident scenario is to present a situation that pushes the team outside of the standard, well-rehearsed procedures for addressing abnormal plant conditions. To achieve this, we created scenarios with difficult choices, goal conflicts, or unique situations that present significant challenges to the team's decision-making process.

Further, to create the necessary severe accident conditions in this scenario, it was necessary to postulate numerous traumas, equipment failures, and at times, operator errors. The use of multiple failures was required to thwart the safety and defense-in-depth design employed in nuclear power plants and reduce the effectiveness of current emergency operating procedures (EOPs).

The boundary conditions of the scenario were constructed for the sole purpose of maintaining a difficult decisionmaking situation for as long as possible. Therefore, it should not be construed that these scenarios are likely events, or even the most likely severe accident sequences. The probability of these scenarios occurring is within, or beyond, the range of traditional IPE core-damage frequencies.

\section{Scenario Objective:}

The objectives of this scenario are as follows:

- To place the panel in a situation where both the vessel and the containment have failed before any core damage has occurred.

- To determine which strategies are employed by the panel to minimize the impact of the accident.

\section{Scenario Assumptions:}

These assumptions are for the presenter and are not to be presented to the panel:

- Plant operating at normal full power conditions.

- DBA steam-line break has occurred that rapidly depressurized the vessel. The break cannot be isolated and remains uncovered when the vessel is re-flooded with low-pressure ECCS systems. An electrical penetration has failed in the drywell region, which allows the drywell atmosphere to be vented directly to the reactor building.

\section{Progression of Events}

0 min DBA steam-line break accident, RPV rapidly depressurizes, drywell temperature rapidly increases, suppression-pool temperature rapidly increases.

Containment isolation, but high area temperatures are reported in an area outside the containment, which would correspond to a containment breach in the drywell area.

$5 \mathrm{~min}$ The reactor water level is restored to normal level with the low-pressure ECCS systems. 


\section{Statement for Panel:}

Approximately 1 hour ago the plant experienced a DBA-type LOCA. The RPV rapidly depressurized and the suppression pool and drywell temperatures increased. High temperatures were detected after the blowdown in the reactor building in an area that would indicate that a penetration has failed in the drywell region of the containment. The operators declared a site emergency and the TSC and EOF were activated.

Currently the water level in the RPV is near the normal mark with level being sustained via the low-pressure ECCS systems. During the course of the accident, neither the drywell nor the wetwell sprays have been activated.

\section{Recommendation:}

At this time in the event an engineer in the TSC makes the following recommendations:

"We need to make some decisions regarding this problem. First of all I believe that the core itself is well protected at the moment, but we may need to be concerned about long-term cooling if the reactor building environment becomes too harsh. This equipment has not been qualified to the same level of environmental conditions that equipment inside the containment has been, yet it is being exposed to a similar environment. Second, although there seems to be no gross fuel damage, we have detected some Xe and Kr gas from our PASS system, which indicates that some cladding has ruptured and gap fission product release has occurred."

\section{Initial Questions to Panel:}

- What are your recommendations for this situation?

- What methods can be employed to minimize the harsh reactor building environment?

- What are the concerns regarding maintaining ACC?

- What can be done to minimize the release of radioactive material?

\section{Background Material for Conducting Exercise:}

\section{Current Plant Conditions}

- RPV Pressure: 15 psig

- RPV Level: normal

- RPV Power: downscale

- DW Temp: $240 \mathrm{~F}$

- DW Pressure : 15 psig

- SP Temp: $190 \mathrm{~F}$

- H2 conc: downscale

- O2 conc: downscale

- Essential DC: available

- AC power: available

- ECCS: turbine-driven systems isolated, motor-driven system available

- PASS indicates some Xe, Kr present

- Assumptions: The reactor vessel has experienced a steam-line break that cannot be isolated. Also, the containment has experienced a failure of an electrical penetration in the drywell region. Some fuel failure has occurred, which has resulted in release of $\mathrm{Xe}, \mathrm{Kr}$ to the drywell. 


\section{Connections to Decision-Making Model:}

\section{Interpret Current State:}

- Knowledge of plant-specific condition and system availability: After presentation of the scenario background, do the panel members realize that:

- the plant has experienced a steam line break

- the drywell has a penetration failure

- radioactivity is present in the containment

- all ECCS systems are available

\section{Determine Implications:}

- Knowledge of accident progression: Do the panel members realize that:

- the core has ACC for the moment

- the containment has adequate heat removal

- the reactor building is receiving mass and energy from the containment

Plan:

- Do the panel members realize that:

- the containment leak is providing a harsh environment in the reactor building

- the leak is transporting radioactivity into the reactor building

- the harsh environment in the reactor building may cause equipment failure that could affect ACC

- the harsh environment may preclude personnel from entering the reactor building

- Do the panel members have a plan to deal with this situation?

- Knowledge of specific plant systems required for implementing plan or procedure: Do the panel members know:

- ways to minimize the gas flow to the containment

- ways to minimize radioactivity to the containment

- ways to minimize the potential damage to ECCS equipment in the reactor building 
BWR-6: RPV Failure at High Pressure

\section{Warning:}

This scenario is intended to be used as a tool or exercise for generating a response from a small accidentmanagement team (or panel) to a hypothetical severe accident. The function of this.accident scenario is to present a situation that pushes the team outside of the standard, well-rehearsed procedures for addressing abnormal plant conditions. To achieve this, we created scenarios with difficult choices, goal conflicts, or unique situations that present significant challenges to the team's decision-making process.

Further, to create the necessary severe accident conditions in this scenario, it was necessary to postulate numerous traumas, equipment failures, and at times, operator errors. The use of multiple failures was required to thwart the safety and defense-in-depth design employed in nuclear power plants and reduce the effectiveness of current emergency operating procedures (EOPs).

The boundary conditions of the scenario were constructed for the sole purpose of maintaining a difficult decisionmaking situation for as long as possible. Therefore, it should not be construed that these scenarios are likely events, or even the most likely severe accident sequences. The probability of these scenarios occurring is within, or beyond, the range of traditional IPE core-damage frequencies.

\section{Scenario Objective:}

The objectives of this scenario are as follows:

- To place the panel members in a core-melt situation for which the existing emergency operating procedures (EOPs) may be challenged.

- To present technical problems that challenge the panel members to decide how to protect the containment integrity if RPV failure is imminent.

- To determine how the panel members resolve post-vessel failure issues.

\section{Scenario Assumptions:}

These assumptions are for the presenter and are not to be presented to the panel:

- The plant is operating at normal full power conditions.

\section{Progression of Events}

0 min Inadvertent MSIV closure causes a turbine trip, reactor scram, MSIV isolation, and a feedwater trip.

$1 \mathrm{~min}$ Operator attempts to manually initiate high-pressure injection systems without success.

RPV inventory decreases by boil-off to suppression pool via the SRVs. Attempts to depressurize the RPV fail, including opening SRVs, ADS, and attempting to draw off steam from the MSIV leakage-control system and the turbine-driven ECCS systems. Operators declare general emergency; TSC \& EOF are activated

$60 \mathrm{~min}$ RPV water level at TAF. 
95 min RPV level below Minimum Steam-Cooling Level.

$122 \mathrm{~min}$ Control-blade melting begins $(2600 \mathrm{~F})$.

$125 \mathrm{~min}$ Melting of fuel cladding begins (3365 F). Operators successfully open 1 SRV.

132 min Fuel melting begins (4870 F).

138 min SRV fails closed.

\section{Statement for Panel:}

A little over two hours ago, the plant, while operating at normal full power conditions, experienced an inadvertent MSIV closure causing a turbine trip, feedwater pump trip and reactor scram that was verified by the operators. Since the reactor scram, it has been impossible for the operators to establish a means to inject water into the RPV. All attempts to depressurize the vessel have failed. Low-pressure injection systems are available and RHR suppression-pool cooling was initiated. The operators were successful in opening one SRV for a short period of time, but approximately thirteen minutes later the SRV closed. When the SRV was opened by the operator, large amounts of radioactivity and hydrogen were detected in the containment. At this time, the operators secured the suppression-pool cooling system for fear of pumping contaminants outside the containment and possible fouling of the RHR heat exchangers.

All level indications appeared to be off-scale/down-scale and less than three SRVs were open. Operators decided that the water level in the RPV was unknown per the EOP wording and commenced with containment flooding. When the TSC was staffed, this decision was countermanded because the RPV was still at pressure. The water level in the wetwell is above the high LCO limit.

Currently, all SRVs are closed except for intermittent opening of one at its safety setpoint.

\section{Recommendation:}

At this time in the event an engineer in the TSC makes the following recommendations:

"We need to make some decision quickly regarding our situation. I see the situation this way: if we are unsuccessful in establishing injection into the vessel, the vessel will fail. To establish injection in the vessel at this stage requires either the ability to depressurize the vessel below the shut-off head of our low-pressure pumps, or re-establish the capability of our high-pressure injection systems. I believe the chances of accomplishing either in our time frame is small.

Therefore, although we should try to accomplish the above tasks, I believe we need to prepare for the vessel failure situation. Our fundamental question is whether to activate the DW sprays prior to vessel failure or wait until after the RPV depressurization event."

\section{Initial Questions to Panel:}

- What are your recommendations for DW sprays?

- Should the sprays be used from an internal or external source?

- What are your concerns regarding pumping contaminants from suppression pool?

- Are there concerns regarding DCH?

- Will water on the floor and in the air aggravate the DCH?

- steam explosions

- short-term oxidation 
- fuel/air heat transfer

- hydrogen burn

- What are the long-term effects from DW spray activation?

- core concrete interaction

- non-condensable generation from concrete ablation

- What would you do if, after RPV failure, the DSIL curve is violated?

\section{Background Material for Conducting Exercise:}

\section{Current Plant Conditions}

- RPV Pressure: 1250 psig

- RPV Level : downscale

- RPV Power: downscale

- DW Temp: $145 \mathrm{~F}$

- DW Pressure : 3 psig

- SP Temp: $110 \mathrm{~F}$

- H2 conc: 5\%

- O2 conc: downscale

- DC Power: available

- AC power: available

- High-pressure injection systems unavailable

- All motor-driven low-pressure injection systems available

- PASS: $\mathrm{Xe}, \mathrm{Kr}, \mathrm{CsI}, \mathrm{CsOH}$

- Assumptions: Reactor has experienced a transient in which the containment has isolated, but no high-pressure injection systems are available. Also, the reactor cannot be depressurized, which will lead to a high-pressure melt scenario.

\section{Connections to Decision-Making Model:}

\section{Interpret Current State:}

- Knowledge of plant-specific conditions and system availability: After presentation of the scenario background, do the panel members realize that:

- the reactor cannot be depressurized

- no high-pressure injection system is available

\section{Determine Implications:}

- Knowledge of severe accident events and their symptoms: Do the panel members recognize that:

- this BWR will experience a high-pressure melt ejection-like event

- whether the PWR DCH issues are applicable

- whether debris is coolable in the containment

Plan:

- Knowledge of criteria for applying HLA (condition of applicability): Do the panel members know:

- the benefits of water on the drywell floor

- the consequence of a steam explosion

- the applicability of a DCH analysis 
- Knowledge of specific plant system required for implementing plan or procedure. - can the panel devise a strategy to minimize the high-pressure failure case?

- Ability to prioritize action when implementing response: Does the panel recognize: - the need for water on the drywell floor

- that containment flooding takes time

- that containment flooding has serious drawbacks

- Ability to identify specific markers/cues given a diagnosis: Do the panel members recognize that: - if the reactor remains at pressure, vessel failures will be identified from containment instrumentation - debris cooling in the containment will be the major mission after vessel failure 


\section{C.3 Glossary for BWR Scenarios}

$\begin{array}{ll}\text { AC } & \text { alternating current } \\ \text { ACC } & \text { adequate core cooling } \\ \text { ADS } & \text { automatic depressurization system } \\ \text { APRM } & \text { average power range monitors } \\ \text { ARI } & \text { alternate rod insertion } \\ \text { ATWS } & \text { anticipated transient without scram } \\ \text { BITT } & \text { boron injection initiation temperature } \\ \text { BWR } & \text { boiling water reactor } \\ \text { CS } & \text { core spray } \\ \text { CS } & \text { cesium } \\ \text { CSI } & \text { cesium iodine } \\ \text { CSOH } & \text { cesium hydroxide } \\ \text { CST } & \text { condensate storage tank } \\ \text { DBA } & \text { design basis accident } \\ \text { DC } & \text { direct current } \\ \text { DCH } & \text { direct containment heating } \\ \text { DG } & \text { diesel generator } \\ \text { DSIL } & \text { drywell spray initiation limit } \\ \text { DW } & \text { drywell } \\ \text { ECCS } & \text { emergency core cooling system } \\ \text { EOF } & \text { emergency off-site facility } \\ \text { EOPs } & \text { emergency operating procedures } \\ \text { F } & \text { fahrenheit } \\ \text { FSAR } & \text { final safety analysis report } \\ \text { FW } & \text { feedwater system } \\ \text { H2 } & \text { hydrogen } \\ \text { HCTL } & \text { heat capacity temperature limit } \\ \text { HCU } & \text { hydraulic control unit } \\ \text { HP } & \text { high pressure } \\ \text { HPCI } & \text { high-pressure coolant injection } \\ \text { HPCS } & \text { high-pressure core spray } \\ \text { HPSW } & \text { high-pressure service water } \\ \text { I } & \text { iodine } \\ \text { IC } & \text { isolation condenser } \\ \text { K } & \text { kelvin } \\ \text { Kr } & \text { krypton } \\ \text { LCO } & \text { limiting condition for operation } \\ \text { LOCA } & \text { loss of coolant accident } \\ \text { LPCI } & \text { low-pressure coolant injection } \\ \text { MPCWLL } & \text { maximum primary containment water level limit } \\ \text { MSIVs } & \text { main steam isolation valves } \\ \text { O2 } & \text { oxygen } \\ \text { PASS } & \text { post-accident sampling system } \\ \text { PCPL } & \text { primary containment pressure limit } \\ \text { PPM } & \text { parts per million } \\ \text { PSIG } & \text { pounds per square inch } \\ \text { RCIC } & \text { reactor core isolation cooling } \\ \text { residual heat removal system } & \text { reactor pressure vessel } \\ & \end{array}$


SLC standby liquid control system

SP suppression pool

SRM source range monitors

SRV safety relief valve

TAF top of active fuel

TSC technical support center

WW wetwell

Xe xenon 


\title{
Appendix D
}

\section{Training Techniques - Research and Discussion}

\author{
Table of Contents
}

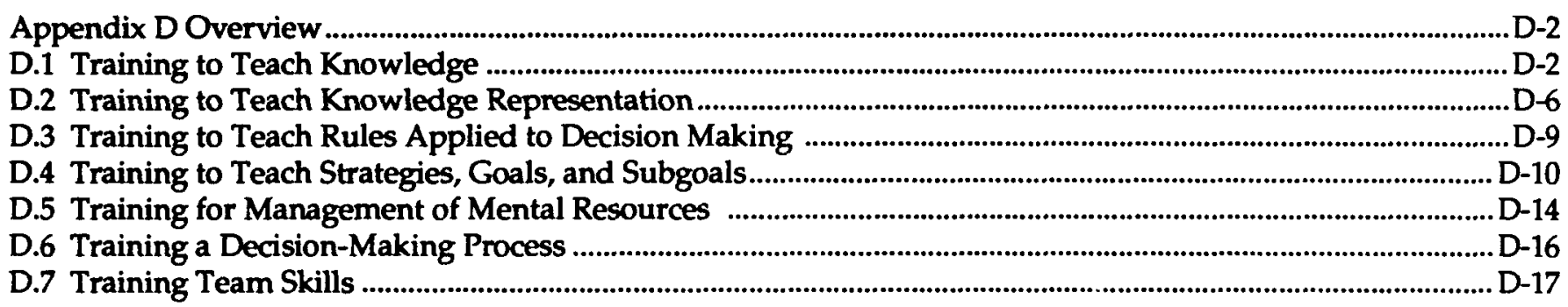

Figures

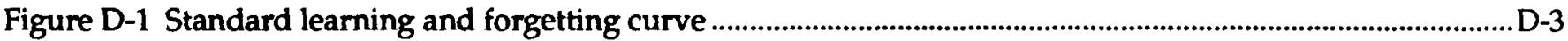

Figure D-2 Standard learning curve for consistently mapped skills ..........................................................................D-15 


\section{Appendix D Overview}

This Appendix accompanies and shares the organizational structure of Section 6.2, which provides descriptions of the 19 training techniques. The information found here provides background and discussion of each technique, including additional examples and references to underlying instructional theory. Also, there are summary sections for each group of training techniques.

\section{D.1 Training to Teach Knowledge}

\section{Use a job or functional context. According to} Druckman and Bjork (1991), there is ample evidence (e.g., Sturges, Ellis, \& Wulfeck, 1981) that a functional context aids a learner in making new knowledge fit more meaningfully into existing knowledge structures. That is, the job context or functional context provides a structure on which to hang new knowledge, which also aids retention. Kieras and Bovair (1984) used a functional context in the form of a functional device model to support the teaching of a procedure to a group of trainees. This technique enhanced retention when compared to a group that used only memorization. The functional context, according to Farr (1987), aids the trainee by furnishing cues to help retrieval and, through the use of a conceptual framework, by facilitating the regeneration of information that may have been forgotten.

Druckman and Bjork identify other reasons why tying task performance closely to the acquisition of new knowledge aids learning and retention. First, making trainees interact with the material-actively doing as opposed to observing passively-has been shown to enhance later performance in many settings (e.g., Johnson et al., 1981). Second, there is a "generation effect" in learning, which refers to the fact that verbal information generated by trainees in response to an instructor cue is better retained than information presented for study (e.g., Slamecka \& Graf, 1978). Finally, the requirement to perform a task and demonstrate the knowledge serves as a feedback mechanism that allows trainees to assess how well they have mastered new knowledge. When trainees understand the gaps they have in their knowledge, they can be more effective in using subsequent information to learn. Thus, the use of performance "tests" makes subsequent training more efficient.

One distinction that is critical to a discussion of knowledge is the distinction between initial level of learning (mastery) and the level of mastery demonstrated at the time performance is required. For example, a trainee may show a high level of mastery of a simple procedure at the end of a twoweek training session, but if that procedure is not used until three months later in the actual job setting, the performance at that time is very likely to demonstrate a reduced level of mastery. Learning level drops off over time when training or practice are not continued (see Figure D-1). Long-term retention refers to the level of performance demonstrated after training has been completed. Thus, there is a distinction between initial learning and long-term retention. The goal is to conduct training so that longterm retention supports skilled task performance months, or perhaps years, after initial learning. This seems especially relevant to training for SAM, since severe accidents are not likely to occur. Druckman and Bjork (1991) review techniques to enhance longterm retention.

2. Use Overlearning. According to Druckman and Bjork (1991), numerous empirical studies have demonstrated that retention is better for overlearned tasks (e.g., Loftus, 1985; Schendel \& Hagman, 1982). More overlearning leads to more enhanced retention. However, several studies (e.g., Bell, 1950; Melnick, 1971) have indicated that overlearning can reach a point of diminishing returns. Thus, increasing the number of supplementary practice trials may not produce proportionate increases in retention. The point at which overleaming on a skill becomes less productive is likely to vary from situation to situation. Therefore, training programs may need to conduct some experimentation to identify the most valuable levels of overlearning.

3. Use distributed practice. The distributed versus massed practice distinction reveals the strong differences that can exist between short-term performance gains (during training) and long-term retention. In general, according to Druckman and Bjork, massed practice produces better performance in initial learning during training but, when compared to distributed practice, produces much poorer long-term retention. Cases have been documented in which distributed practice produced 


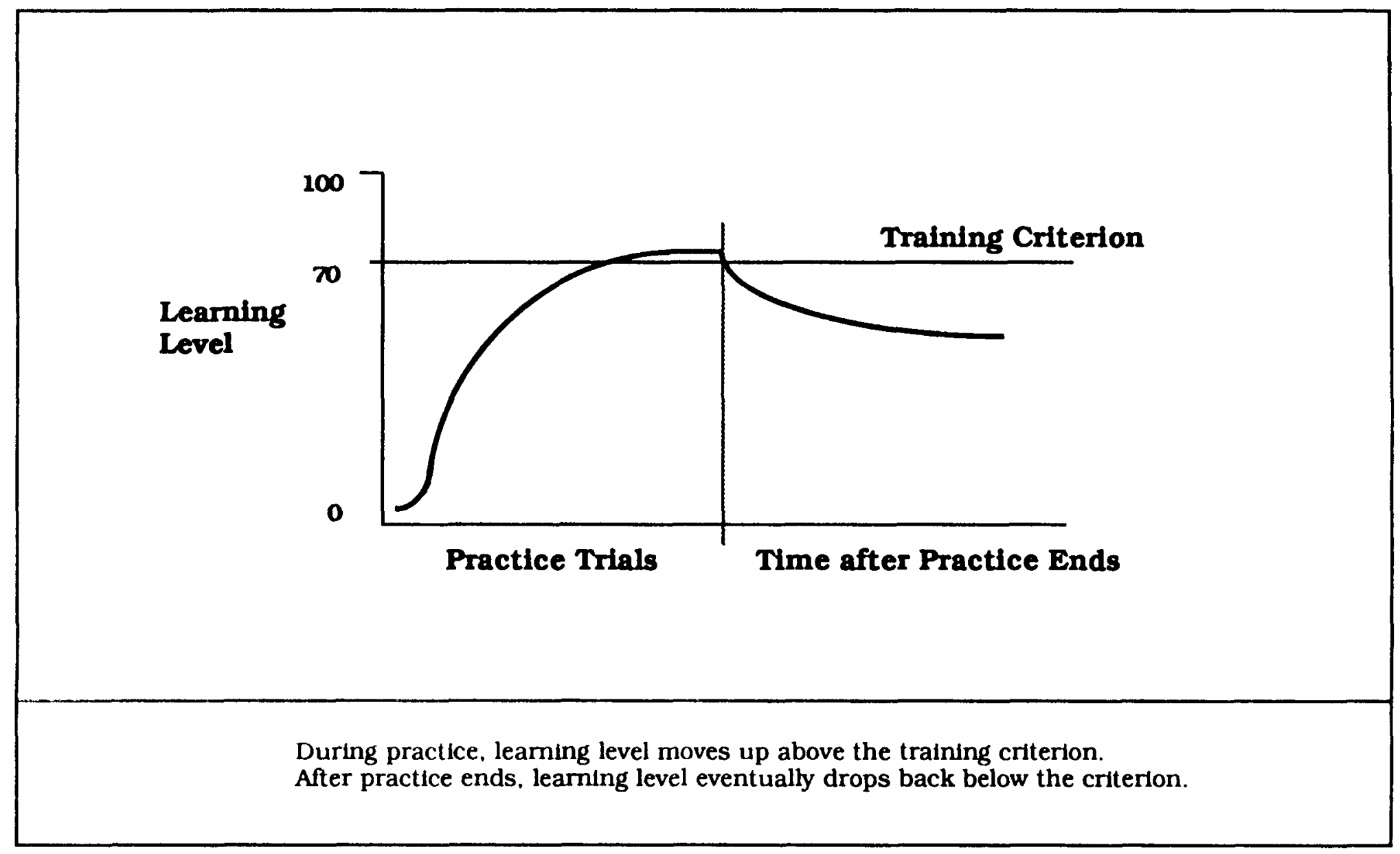

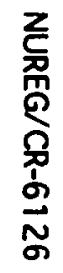

Figure D-1. Standard learning and forgetting curve. 
long-term recall performance twice that of equivalent massed practice (e.g., Bahrick \& Phelps, 1987; Glenberg, 1979; Melton, 1970). In fact, the general phenomenon of superior retention of both verbal material and motor skills with distributed practice is one of the most reliable in human experimental psychology.

Despite the extensive evidence of its success, training programs may not use a distributed practice method for training for two reasons. One reason is that in many cases, massed practice produces better performance or faster learning curves in the short term. The true benefits of distributed practice may not be revealed for months or years, and because of that gap, those benefits are less easily associated with a training method. The second reason that distributed practice is less likely to be enthusiastically embraced is that it takes more time within the training program to reach the same level of learning. If practice sessions are distributed over several weeks instead of massed into a single day, the training of that particular skill, in some sense, is delayed.

4. Use contextual variety. One technique to increase contextual variety is to require trainees to perform other simple tasks during training (e.g., Fendrich et al., 1988; Magill \& Hall, 1990). The secondary tasks produce interference, and trainees must develop multiple processing strategies for overcoming the interference and learning the new knowledge or skill. Other techniques are to mix types of training and to block easy task performance, perhaps by requiring performance to be faster. Schneider (1985) advocates varying the speed-accuracy requirements of a task during practice so that under some training conditions performing quickly is rewarded over accuracy. Some demonstrations (e.g., Smith \& Rothkopf, 1984) have shown that simply varying the instructional setting (e.g., different rooms, different instructors) can enhance retention of new knowledge.

One foundation for this technique is the "depth of processing" phenomenon described by Craik and Lockhart (1972), which states generally that the quality of processing is more important than the duration of processing. For example, Craik and Lockhart demonstrated that word recall is superior when the introduction of words requires thinking about word meaning than when initial encoding requires thinking about word sounds. Increased processing of information creates more links to existing knowledge. Generally, the goal is to have trainees encode knowledge in multiple contexts so that its retrieval does not rely on replicating the exact conditions present at the time of learning.

5. Use cooperative learning and peer teaching. Slavin (1988) points out that this type of instruction works best when the learning or achievement of the entire group is assessed or graded. That is, individual learning goals must become less important than the performance of the group. In evaluating this form of instruction, the measure of performance is often improved retention of factual material, and, overall, a group structure has been found to result in better performance than individual or competitive incentive structures (Sharan, 1980).

Brown and Palincsar (1989) describe the benefits of both the supporting role of groups and the conflict role of groups. In terms of support, the group is more capable than an individual in identifying different points of view and in providing more expertise. However, a more critical function is the group's ability to externalize learning assessment, which is critical to effective learning. The group is more likely to express overtly the need to define a problem, plan a solution, determine whether sufficient data exist to support an hypothesis, and evaluate progress. These are critical elements of many cognitive skills. Because the group must work together, these functions become overt and serve as an effective model for problem solving. Many of the benefits of cooperative learning and peer teaching come from making explicit good models of learning and thinking (see also the discussion of cognitive apprenticeship in Sections 6.2.4 and D.4). Trainees internalize these learning methods over time, as they observe them.

The other major role of cooperative learning emphasizes the value of conflict. Groups can play a skeptic or critic role by asking tough questions and requiring clarification, justification, and elaboration. For example, an individual's understanding of a difficult concept is tested by the group through questions. Then, the individual must develop a sufficiently concise and thorough account to satisfy the group. Through this process, gaps are identified in comprehension, and the understanding of the material becomes deeper and more flexible. This critic role is less likely to occur with the individual on his own. Also, note that groups cannot act autonomously. It is necessary for an instructor to 
provide a format or technique or, in many cases, guidance of the group learning process.

6. Use accelerated learning programs. Some of the commercial accelerated learning programs make extraordinary claims about their effects on learninge.g., "increase learning 5 to $\mathbf{5 0}$ times...requires no effort on the part of trainees...awakens creative abilities." These claims, because they are so provocative, have gotten attention from large institutions involved with training (e.g., Dept. of Defense), and therefore, Druckman and Swets took a careful look at the evidence behind the claims.

Druckman and Swets reached the following conclusions from their examination of SALTT. First, they note that the program incorporates instructional techniques known to be effective and focuses on improving learner and instructor motivation, which is a valid objective for instruction. Despite the proven value of the training techniques described above, training can become routine, and maintaining motivation in both the instructor and trainees is important for effective learning. Second, the nontraditional elements of the SALTT approach-the use of music, the attempts at manipulating mood or state (relaxation, etc.), "whole brain" learning-are not well supported by controlled research. In fact, another section of the Druckman and Swets volume addresses the lack of evidence supporting manipulations to alter mental state for enhanced learning. Finally, although there can be clear benefits from many of the traditional instructional techniques used by SALTT, the provocative claims for enhancing learning through the non-traditional elements are not borne out by experimental tests. While there can be noteworthy improvements in learning, enhancements that approach the claims of several orders of magnitude are not possible.

Summary and conclusions. There has been a great deal of research on the principles and factors that underlie human learning and memory and the design and development of effective instruction. It is not possible to review the entire literature in this report. Instead, significant factors in enhancing learning have been identified. More specifically, the intent of the techniques described here is to make knowledge available in the context of task performance. Thus, the two primary concerns were with tying knowledge and simple skills to the context of job performance and with enhancing long-term retention (as opposed to increasing the speed of learning during training). This last issue is critical since the techniques that are most effective for enhancing long-term retention are also those that can hinder or slow initial learning. Thus, although training can initially seem less efficient, its benefits can be greatly enhanced.

As before, training techniques have been tied back to basic mechanisms of human cognition. One concern is the characteristic learning and forgetting curve shown in Figure D-1. Training techniques can be used to increase the initial learning level or to retard the degree of forgetting that inevitably occurs. The trainer may go so far as to determine the parameters of this curve and adjust training accordingly (e.g., set initial training criterion, schedule refresher training). Other techniques focus on the encoding and elaboration of new knowledge. Several techniques were described that encourage elaboration of new material at the time it is introduced. Because forgetting is tied to an inability to retrieve knowledge in the required context, training techniques attempt to create multiple connections to existing knowledge (i.e., multiple paths to retrieval). Most important is to identify retrieval cues that are present in the job context so that performance of the job triggers the access of relevant knowledge. Also, because learning is an active process, the role of motivation cannot be neglected. Although motivation is often intrinsic to learning, in some cases, extrinsic rewards (free time, special privileges, etc.) can be more effective for motivating trainees. In summary, effective knowledge training is not the result of a single "magic bullet," but the integration and management of multiple techniques and, perhaps, multiple training media.

Sources of further information on this issue:

Druckman, D., \& Bjork, R.A. (1991). In the mind's eye: Enhancing human performance. Washington, DC: National Academy Press.

Druckman, D. \& Swets, J.A. (1988). Enhancing human performance: Issues, theories, and techniques. Washington, DC: National Academy Press.

Farr, M.J. (1987). The long-term retention of knowledge and skills: A cognitive and instructional perspective. New York: Springer-Verlag. 
Montague, W.E. (1988). What works: Summary of research findings with implications for Navy instruction and learning. (Technical Report NAVEDTRA 115-1). Pensacola, FL: Office of the Chief of Naval Education and Training.

\section{D.2 Training to Teach Knowledge Representation}

7. Train perceptual patterns. Skilled performance often relies on extracting the relevant information from the world and ignoring or bypassing information that does not currently have relevance. The ability to identify meaningful organizations of information supports two aspects of performance. First, the initial task representation that is developed is strongly influenced by an understanding of meaningful patterns. Experts see the world through a filter that breaks up, or parses, the world into meaningful objects and events. For example, studies of chess skill (Chase \& Simon, 1973) show that chess masters readily identify strategic relationships among chess pieces when a game situation is presented. It is estimated that masters can identify tens of thousands of meaningful chessboard configurations. Similarly, Means et al. (1988) found that expert air traffic controllers represented the aircraft in their airspace in terms of functionally related groups. In many cases, this representation placed aircraft that were in close proximity into different groups, showing that functional relations were more important than distance in the three-dimensional space. Thus, trainees need to learn the functional groups that help them represent the task.

Second, certain patterns of information (e.g., visual patterns, auditory patterns, combinations) indicate that a specific response is required. Simple cues to respond are auditory alarms. However, experts can become sensitive to more complex cues that guide behavior. For example, there may be patterns of indications and alarms that guide higher levels of operator skill (e.g., for regulating a process). In some cases, the perceptual patterns are difficult to describe-for example, explaining to a student driver when to begin turning the steering wheel in an intersection, or explaining a sound to your mechanic that you believe is indicative of an engine problem. Skilled practitioners acquire these patterns, and it is important to make them available to trainees. Two examples illustrate available techniques.

Vidulich, Yeh, and Schneider (1983) altered the timecourse of an air intercept control task to make a perceptual pattern more salient to trainees. In this" task, the controllers' job is to give friendly aircraft a directional heading that will allow them to gain a superior air position over an enemy aircraft. In highfidelity simulations of the task, there is a delay of several minutes after a heading is given before the controlled aircraft achieves its final position. Because of this delay, controllers had a difficult time modifying their judgments to improve performance. Vidulich et al. compressed time in the simulation after the controller's judgment had been made to provide for quicker and much more effective feedback. Under this training condition, the trainees could see the flight pattern easily and use it to guide their judgments in later trials.

Flight skills often rely on learning complex visual cues that are virtually impossible to describe in words. Lintern and Roscoe (1980) aided trainees in isolating the appropriate visual cues by indicating when the cues were present. Lintern and Roscoe presented a realistic visual display while training landing skills to novice pilots in a flight simulator. The trainees practiced landing the simulator over many trials. When pilots were outside the boundaries of the glideslope (landing path), the display was augmented (artificial cues were added to the display) to direct them back. When the pilots were within the glideslope, the artificial cues were removed. With this technique, trainees can learn to identify the cues that are present when their performance is accurate.

Further, this technique ensures that as trainees gain expertise, they receive less artificial feedback and the training situation more closely resembles the actual task. An alternative technique was to augment the display when performance was within bounds. However, this method can lead trainees to become too reliant on the artificial cues for landing and fail to develop the ability to land without those cues.

8. Train mental models. Mental models provide a deeper description of the knowledge required by performers. As stated in Chapter 2, mental models are complex representations that allow one to simulate mentally a system or process in order to reason about cause and effect, consequences of actions, feasibility of control actions, effects of 
malfunctions or failed components, etc. Rouse and Morris (1986) provided the following definition: "Mental models are the mechanisms whereby humans are able to generate descriptions of system purpose and form, explanations of system functioning and observed system states, and predictions of future system states." Generally, three different classes of mental models have been described: physical, functional, and causal. First are models of the physical or mechanical aspects of a device or system. These models provide knowledge about the parts of devices and how devices work (e.g., a pump, a turbine). The second category are functional mental models, which capture the functional relationships among devices. This distinction is important because similar or identical devices can serve very different functions in a complex system. The third type of mental model, and the one that has received most attention in training, are causal models, or sometimes called qualitative reasoning models. These models allow one to reason about causation within the system-e.g., if this component changes state, how will that affect other components? Causal models are valuable for predicting system performance, troubleshooting, and other problem-solving activities.

It is critical for trainees to integrate the various types of knowledge that are required for mental models. A means to reason about system operations is clearly necessary. In fact, White and Frederiksen (1986) believe that a primary reason for training mental models is to provide trainees with the ability to reason qualitatively about a system--that is, to first develop a sense of what is occurring and then quantify the effects. They point out that studies of expertise have shown that skilled problem solvers develop problem representations at a qualitative level before doing a quantitative analysis (Chi et al., 1981; Larkin et al., 1980). For example, in physics problem solving, skilled problem solvers typically create a free-body diagram that shows the forces acting on an object and reveals the overall effect. This representation is then used to select and solve equations that produce a quantitative answer. Less skilled problem solvers are more likely to select equations for solution in a less disciplined manner. That is, they may begin solving equations that seem to include the relevant unknowns until they find a way to obtain the unknown specifically requested. Other investigators (deKleer, 1985) have observed analogous approaches in electronic circuit design and analysis. Performers require an accurate mental model of the system to support this type of reasoning process.

Another primary reason to train mental models is to guide trainees in developing useful and effective representations of the system. Unaided, trainees will develop a system representation, but that representation may contain misconceptions about the system, inaccuracies, or gaps. For example, in the ISLOCA scenario described in Section 3.1, it became clear that not all operators knew there was a connection between the RHR and the PRT that could account for the PRT rupture. White and Frederiksen (1986) also point out that specific mental models support reasoning on some problems but not on others. For troubleshooting or diagnostic tasks, different perspectives may be required to solve the problem most efficiently. In troubleshooting circuits, a voltage drop perspective is useful, but sometimes a current flow perspective is more useful. Similarly, in nuclear power plants, different perspectives on the system (e.g., pressure, mass, temperature) become more or less useful for reasoning about a fault or considering the implications of a control action.

The White and Frederiksen technique is intended to lead trainees through a progression of models, beginning with simple models and progressing to more comprehensive and more complex models. They use two key concepts to describe mental model complexity in the area of electronic circuits. Mental models have orders and degrees. The order of a mental model determines the types of inferences that can be drawn about changes to system components. Zero-order models can be used to make determinations about presence/absence or on/off states of components: If component $x$ changes state will component $y$ be on or off? First-order models can be used to determine whether there will be an increase or decrease in a component parameter, and second-order models provide information about the rate of change in component parameters. Added to this distinction is the notion of degree of elaboration, which refers to the number of rules, elements, or constraints that are included in the model. As more rules are added, the model becomes more complex.

Within the White and Frederiksen QUEST learning environment, trainees are given electronic circuit problems to solve through a circuit simulation tool. Trainees engage in making predictions about the circuit after components have been added or changed, 
identifying possible faults, designing circuits to achieve some purpose, and troubleshooting known circuit faults. Through a number of activities, trainees eventually transform their model. This transformation can occur through the addition or refinement of knowledge or rules, a generalization of rules, a differentiation of rules, or in some cases, a shift to an alternative model. This transformation or progression of models is supported with four types of learning activities: Open-ended exploration is a means for trainees to construct and manipulate circuits, explore their behavior, and request explanations for the observed behaviors. For example, trainees might change the state of a device or introduce a fault to observe the consequences. Problem-driven leaming occurs when the system selects problems for trainees to solve. The problem selection is designed to push trainees into situations that require a transformed model. Although trainees are initially required to attempt a solution, they can request explanations when they run into difficulty. A third activity is example-driven learning. In this case, the system presents and solves example problems, and then presents similar problems for the trainee to solve. The system presents elaborated examples by explaining the solution; it articulates the goals, subgoals, and strategies it uses in solving the problem. Finally, transformation can be supported through trainee-directed learning, in which the system informs trainees about the instructional objectives underlying problem sets. For example, trainees can select problems that allow them to learn a new concept. Therefore, in some sense, trainees set their own instructional goals.

These four learning activities provide a rich environment in which to develop a complex representation of the system. An earlier project, called STEAMER (Hollan, Hutchins, \& Weitzman, 1984), also used a graphical simulation to support the training of mental models. STEAMER provided a simulation of a steam propulsion system as an "interactive, inspectable graphical simulation." The two primary learning activities in STEAMER were, in White and Frederiksen's terms, open-ended exploration and example-driven learning. For exploration, trainees were given a computer-based "mini-laboratory" in which they could assemble and test sets of components. The problem examples presented by the instructional system, as in QUEST came with explanations.
Summary and conclusions. A number of computerbased learning environments have been developed to aid in the training of mental models of complex systems. In addition to the QUEST and STEAMER programs, there have been other large efforts, such as SOPHIE, which is directed at teaching electronics (e.g., Brown, Burton, \& deKleer, 1982). One intent of these learning environments is to provide a realistic system simulation that allows trainees to formulate, test, and observe consequences of interactions between system components. These learning environments support exploration of system characteristics, and they provide problem-solving exercises and examples that require trainees to reason qualitatively about the system. Early versions of STEAMER relied solely on the power of an "interactive and inspectable" simulation. However, more mature systems are not simply exploration tools. A critical component in each is an explanation capability that can serve as an articulate expert. QUEST, within its many learning activities, guides training in that it provides the trainee with the capability to have the simulated system explained: the observed behavior of the experimental configuration is explained; the example troubleshooting problem is explained; and if the trainee fails to solve a problem presented to him, the "expert" shows and explains the solution.

The guidance within QUEST seeks to move the trainee through a systematic progression of models. As the model acquires more rules or refines existing rules, it can solve a wider set of problems. It may also develop trainee's capability to take multiple perspectives on the simulated system, which is another tool for bringing flexibility into the qualitative reasoning process. The expert component demonstrates skilled reasoning in a concrete, problem-solving context and, therefore, provides a model of performance for the trainee. Note that this articulate expert is not required to be an artificial intelligence element of the learning environment. It could also be a human instructor who monitors a trainee involved with the simulation. No matter the technology, the instructional role is important.

The final point to emphasize is the effort that White and Frederiksen have put into determining how to develop a progression of mental models. The task is not straightforward. Early models need to have a number of characteristics. First, they have to support some level of reasoning to allow trainees to solve 
problems. Second, they have to be simple and yet prevent misconceptions about the simulated system. Simplification, when not applied carefully, can introduce incorrect notions of system performance. Later, more complex models may need to be supported by a more complex simulation environment (see discussion in Section 6.2). Methods for developing a progression of mental models need to consider the reasoning process of skilled performers or the reasoning process that is appropriate to the operations task. This latter process includes the set of procedures that guide operations.

\section{D.3 Training to Teach Rules Applied to Decision Making}

9. Identify and eliminate buggy rules. An area that has been received thorough scrutiny in the analysis of buggy rules is subtraction errors among young students. Researchers (e.g., VanLehn, 1982) have uncovered on the order of 100 buggy rules that are employed by students. One example is the alwaysborrow-left rule, shown here:

$$
\begin{array}{r}
65 \\
-19 \\
\hline 46
\end{array}
$$$$
\text { (B) } \begin{array}{r}
365 \\
-109 \\
\hline 256
\end{array}
$$

In problem (A), the student has correctly borrowed from the left column to convert 65 to 5 tens and 15 ones. Likewise, in (B), the student has borrowed from the tens column to turn the 65 in 365 to five tens and 15 ones. The result of 256 is correct. However, some students, when trying to generalize a rule learned from a two-column problem, such as (A), to a threecolumn problem, form the incorrect rule "if the top value in the right column is smaller than the bottom value, then borrow from the left-most column." Thus, in (C), the borrowing was done from the 3 in the hundreds column instead of the 6 in the tens column. 365 becomes 2 hundreds, 6 tens, and 15 ones, and the result is 166 , an incorrect response.

The empirical work on buggy rules led to a theory, called Repair Theory, about the acquisition of procedures and rules (Brown \& VanLehn, 1980; VanLehn, 1988). Repair theory's primary claim is that learning occurs at an impasse. An impasse is a point at which the trainee has no rule for progressing. When the trainee reaches this impasse, say in the midst of a subtraction problem, he must either be given the correct rule by an instructor or create a rule to bridge the impasse. Buggy rules can appear at these impasses when aid is not available. The implication for training is that it is critical to supply the correct rule to bridge the impasse before the trainee creates a bad rule. This is only possible when the trainee asks for help at the impasse. Thus, instructors need to provide immediate feedback when errors occur to prevent the buggy rule from being strongly acquired. This notion of immediate feedback is also a critical element of Anderson's approach to instruction, as revealed in the next section on production rules.

10. Train production rules. The LISPITS tutor assumes several roles in teaching LISP programming. Initially, the tutor presents the general form of the code (a template to identify goals) and provides certain syntactic structures that may be overlooked by the trainee. In some sense, these are working memory supports. The primary role of the tutor is to diagnose errors and intervene when the trainee commits an error or is stuck. When the trainee types an inappropriate bit of code, LISPITS intervenes immediately to provide an explanation of why it is incorrect. The trainee is then given the opportunity to try again. If the responses continue to be incorrect, LISPITS continues to diagnose and explain why the response is incorrect. If the trainee continues to input incorrect code, the appropriate response will be supplied. If the trainee proceeds through the exercise without error, the tutor's role is minimal. When the trainee has completed the code, he is asked to run the code and watch it operate to conclude the exercise.

The primary focus of LISPITS is to aid the trainee in acquiring the set of production rules underlying LISP programming, and it is intended to represent a set of instructional principles (Corbett \& Anderson, 1992). Specifically, six principles guide the tutor's structure and behavior:

1. Communicate the goal structure underlying the problem-solving task because problemsolving is goal-driven.

2. The knowledge to be taught and the model of the trainee should both be described in terms of a set of if-then production rules.

3. Provide instruction in the problem-solving context, and let trainee's knowledge develop 
through successive approximations of the target skill.

4. Provide immediate feedback on errors to prevent incorrect rules from developing.

5. Increase the size of the task being presented as learning progresses.

6. Minimize working memory load.

Summary and conclusions. Like other training techniques, the techniques described here begin with a detailed description of skilled performance. In the cases discussed, fairly simple procedural skills (mathematics or programming) were analyzed into a set of if-then production rules. When trainees acquired the complete set of rules and eliminated the buggy rules, they could perform the task. A second important element of this technique is the identification of incorrect rules. The Repair Theory provides an account of how learners can acquire incorrect rules in the course of learning. The BUGGY tool allowed teachers and other evaluators to abandon acquired notions of errors and to adopt the perspective that errors are often the result of applying buggy rules in a systematic way.

As the section on strategies and goals shows, some of the lower-level guidelines developed here are not universally accepted. For example, the use of immediate feedback on errors is not part of other intelligent tutoring systems. Also, note that this technique, by casting performance in terms of if-then rules, provides a much more fine-grained approach to describing performance than do other approaches.

\section{D.4 Training to Teach Strategies, Goals, and Subgoals}

11. Use cognitive apprenticeship. At the highest level of description, cognitive apprenticeship presents a model of skilled performance, supports practice of the task, and encourages comparisons of the student's performance to the model. A critical element to this technique is the model of skilled performance presented to students. Palincsar and Brown (1984) have developed a model of skilled reading that reveals the cognitive activities underlying comprehension. Skilled readers use a number of activities to verify and extend their understanding of the text. Palincsar and Brown use four activities to guide reading practice: summarizing the main points of the text, formulating questions about the text, clarifying more specific points or elements of the text, and predicting how the text will continue. In the Palincsar and Brown technique, teachers and students work together on a paragraph or short section of text. Initially, the teacher models the process of summarizing, formulating questions, etc. Eventually, the teacher requires the students to take an active role in the process. The teacher supports students by coaching and critiquing their performance, perhaps providing hints or prods when needed. This activity enables students to remain engaged for the entire comprehension process. Often, the critical element of support is providing the subgoals, or a control structure, for the process (e.g., "OK, you've completed this element of the process, now move to the next element"). By relying on this "scaffolding" type of support, students are able to work through the process and read with greatly improved comprehension. As students become more proficient, the teacher's support fades. Eventually, students take on the complete process.

A similar technique has been developed for teaching writing skills (Scardamalia and Bereiter, 1985) and math skills (Schoenfeld, 1985). As with reading, the process that underlies writing is developed and modeled for the students in Scardamalia and Bereiter's technique. Often, young students faced with a writing task will simply list everything they know about a topic. The process modeled for students encourages them to make their writing more purposeful by moving through stages of generating ideas, improving ideas, elaborating ideas, identifying the goals of the composition, and developing a cohesive whole. Good writers consider their audience, determine how and when to buttress main points, adopt a style or voice, and use other strategies to communicate effectively through writing. This process is not often taught explicitly, and students learn at several levels by observing skilled performance. First, the cognitive process is demonstrated as goals, subgoals, and strategies for developing a well written text. Second, students observe the obstacles that skilled performers can encounter in difficult problems and the way those obstacles are overcome.

Cognitive apprenticeship techniques often employ groups of students with the intent of developing the roles of both producer and critic. As a student begins to read or write by adopting the process presented by 
the teacher, other students can evaluate how well the student is performing. The ability to evaluate performance is a critical element of this technique because in the process of critiquing, students are forced to articulate their knowledge about how the process should be carried out (e.g., what makes a good summary). In Schoenfeld's (1985) approach to math instruction, this element becomes formalized as a final component of the instructional process, called a post-mortem. First, the teacher recapitulates the solution of the problem, highlighting the general aspects of the process. Collins, Brown, and Newman refer to this as an "abstracted replay" that is intended to focus attention at a higher-level description of the solution. Students also become involved in the postmortem to improve their self-monitoring and selfdiagnosis skills.

The formal model of cognitive apprenticeship presented by Collins, Brown, and Newman is more comprehensive than the specific techniques developed for reading, writing, and mathematics. Specifically, they identify six elements. Modeling is a set of techniques the teacher uses to demonstrate or reveal the cognitive process underlying performance. The teacher typically solves problems and makes explicit the cognitive skills (strategies, subgoals, goals) that guide the process. Coaching comprises a set of techniques the teacher uses to aid a student in performing the task. The teacher may offer hints, give feedback, or prod with reminders. Coaching also can include the selection of tasks that enhance instructional opportunities for a student. Scaffolding is the teacher's means for reducing the student's performance requirements. In some cases, physical devices such as cue cards are used to structure a task. Over time, the teacher's support fades as the student becomes more capable. A fourth element is Articulation, which is a set of techniques to force students to articulate their knowledge, reasoning, or problem-solving process. One technique was to place other students in the role of critic. Articulation leads to Reflection, which enables students to compare their performance to the model of skilled performance or to the performance of another student. Reflection techniques are effective when they allow the student to abstract up a level and consider the more general form of their thinking process (e.g., abstracting the general strategies). Finally, students are encouraged to engage in Exploration, which is a means to test their newly developed skills. Students can begin to define their own learning tasks (e.g., take on new writing assignments) or to explore new domains. The goal is to find challenging tasks that students will find interesting and will be motivated to attempt.

12. Use coached practice environments. Two primary examples of this technique are the WEST project (Burton \& Brown, 1979) and the more recent SHERLOCK project (Lesgold et al., 1992). Other examples can be found in LOGO (Papert, 1980) and WUSOR (Goldstein, 1980).

WEST (Burton \& Brown, 1979) uses a boardgame called "How the West was won" to involve young students in simple mathematical operations. Players of the game must move their gamepiece along a numbered line to reach a town at the end of the line. The first player to reach the town is the winner. When a player's turn comes, he is given three numbers from the three spinners on the computerbased gameboard. For example, he may get the numbers $2,2,3$. Using these numbers, the student must compose an arithmetic expression that involves two different operators to determine the number of spaces he can move (e.g., $2+2 * 3=8$ spaces; $2+2-3=1$ space). The game is designed so that the highest number is not always the best move. Other objectives for moves are to bump an opponent backward or to land on a space with a shortcut to a higher space. Thus, students may have a desire to achieve a specific number. It was noticed that students involved in this game fall into fixed patterns of combining numbers and miss opportunities for better moves unless they were coached to try new combinations.

WEST coaches students in an opportunistic manner using an expert model. When a student is given three numbers for a move, WEST evaluates all combinations of those numbers and determines the best moves possible, using a particular strategy. When the student selects a combination of numbers, WEST analyzes how well the student is applying the expert's strategy. For example, the student may select a combination that does not take advantage of bumping the opponent. At this point, WEST will intervene with a description of the value of bumping and a concrete example of how the numbers could be used to bump in this situation. WEST also ensures that students are executing the basic mathematics correctly. More generally, WEST keeps track of the skills that the student has demonstrated and the ones that seem to be missing. Each skill is then presented 
to the student through coaching at the point the student fails to apply it.

WEST's goal is to make coaching relevant and memorable by introducing advice in a very specific context. Thus, the model of expertise is constantly compared to the performance of the student to determine the elements of cognitive skill the student is missing. The WEST coach is also guided by a larger set of pedagogical principles that aid in determining when and how to intervene (e.g., do not tutor on two consecutive moves). It is also possible for the student to request hints before selecting a combination strategy, and WEST can present hints at several levels, which become progressively more directive.

SHERLOCK (Lesgold et al., 1992), another coached practice environment, is an ITS that has been developed for a complex electronics troubleshooting task conducted by Air Force technicians. As in WEST, instruction is given to students actively engaged in troubleshooting in a computer-based simulation of the actual system. The primary goals of the ITS are to train students to use troubleshooting strategies, establish equipment configurations that permit appropriate tests (mental model), and use test instruments to take measurements. Students are presented with a challenging troubleshooting problem and asked to carry out a complete solution. The primary support students are given are sets of hints. Unlike WEST, SHERLOCK does not intervene (except in a few specific situations). Instead, students request help when they are stuck, and SHERLOCK offers four types of hints. Action hints are provided when the student must determine which test to carry out and how that test is done. Outcome hints provide results of a test, perhaps by reading a meter.

Conclusion hints aid the student in determining the meaning of the test results. And, Option hints help the student determine what actions to pursue next. SHERLOCK decides the type of support to provide based on the student's current activity. In addition to selecting the type of hint, SHERLOCK also determines how explicit the hint should be. Level 1 hints simply recapitulate what the student has done so far. Hint levels 2 through 5 are progressively more explicit or detailed. SHERLOCK selects a hint level based on its model of the student's competence. Students also have available a Panic button, which can be used when the student feels he is lost. The Panic button leads to an overview of the problem that allows the student to step back and think more broadly about the troubleshooting strategy.

As the student moves through the problem, SHERLOCK identifies instructional goals at each point in the problem space. SHERLOCK knows what an expert would do at that point, and it develops expectations about the student based on the student's previous performance at similar points. Again, coaching is related to establishing the appropriate configuration of equipment to run meaningful tests, developing efficient strategies for testing components and isolating the fault, and taking accurate measurements. Further support is provided by SHERLOCK's ability to provide action summaries and prevent students from pursuing components that have already been ruled out or previously tested. This is effective since there are often working memory problems for students involved in a complex troubleshooting task. That is, students have trouble keeping track of the problem at the subgoal level. In initial evaluations, the roughly 25 hours of training available on SHERLOCK gave Air Force technicians instructional experience equivalent to four years on the job.

Coached practice environments, as illustrated by WEST and SHERLOCK, take an approach quite different from the cognitive apprenticeship technique. Generally, students become engaged in problem solving and the tutor monitors them carefully. A key element in this technique is the development of a student model that diagnoses the skills attained by the student and identifies the differences between student and expert performance. In each ITS, the system knows what the expert would do in any situation and diagnoses student performance in that context. WEST intervenes to reduce differences and make explicit the skilled approach. SHERLOCK prepares appropriate advice to get the student back on track when the student falters and requests help. Thus, the model of skilled performance is gradually revealed in the context of actual problem solving, and there is less emphasis on presenting the model of skilled performance as a whole. However, in later versions of SHERLOCK a reflective follow-up has been added at the conclusion of each problem that ties student performance back to the model of skilled performance. This is similar to the reflection phase in the cognitive apprenticeship technique. 
3. Use planning-support environments. GIL is a graphical program-planning environment that allows trainees to build the structure required for a LISP program. A simple programming goal is given to students, and they are required to construct a series of LISP functions to achieve that goal, each time indicating the input and output of the function. Thus, the mechanics of forming actual code are neglected. As with other tutoring systems, GIL uses an expert model to identify the appropriate functions at each point in the programming task. Because multiple solutions are possible, GIL considers a set of strategies. Coaching comes from GIL in two ways. First, the trainee can request a hint from GIL when he is stuck. Hinting first addresses a general strategy or subgoal of the programming task-for example, "try to achieve this subgoal using this function" accompanied by the reasoning. A second request for help results in more explicit information about actual values for the function and its inputs and outputs. The second opportunity for coaching comes when the trainee makes an error, and GIL intervenes with advice. GIL addresses both errors at a lower, syntactic or legal level and at the strategic level. An example of a legal error is a case in which the trainee identifies incorrect outputs for a function. A strategic error occurs when the trainee offers a function that is not useful for achieving the programming goal.

Thus, like other learning environments, GIL provides a task environment and a coaching capability. Like WEST, GIL can be asked for help or can intervene when the trainee makes an error. What is different about GIL is the graphical support tool and the fact that programming is moved up a level from writing actual code. GIL's hints and explanations are fairly lengthy and provide concrete examples of how useful strategies might be applied and the types of subgoals that have value. Therefore, GIL supports the strategic and planning skills not addressed explicitly in LISPITS. Another ITS, BRIDGE, imposes even greater structure than GIL on the planning element of programming.

BRIDGE is a tutor for training PASCAL programming (Bonar \& Cunningham, 1988). Bonar (1985) argues for a means to support students in turning natural language plans into working code. That is, students are typically able to express plans in a non-technical manner. They can generate naive notions about the logic the program should contain. BRIDGE uses progressive stages to evolve these plans into modules of actual PASCAL code. In the first stage, the student constructs a set of step-by-step instructions using English phrases. Next, the student matches these instructions to programming plans and builds a program using a representation of these plans. Then, the student matches the plans to programming language constructs, which are then used to produce the actual PASCAL code solution.

BRIDGE, in supporting this plan evolution, provides students with a graphical representation of the program's elements. This graphical environment allows students to see the entire program and the interactions between its elements. The interface also provides strong constraints on the program's structure and forces students through the multi-stage planning process. Thus, the model of skilled performance comes through the process imposed on students as well as the hints available to students. As with the other ITSs, a model of skilled performance monitors student inputs and identifies errors or gives advice on next moves.

Thus, GIL and BRIDGE are in many ways very similar to the coached practice environments described in the previous section. What sets them apart is their focus on supporting explicitly the planning activities via a graphical support environment in which programs are initially constructed at a higher level. The development of code, which was the focus in LISPITS, is neglected until the construction of effective plans is complete. Moreover, hints and program advice are initially directed at the level of strategies and subgoals. The graphical interface also aids students in keeping track of the entire solution. Recall that SHERLOCK relied on an action summary when students got lost. However, because these programming tasks are much simpler than the troubleshooting tasks of SHERLOCK, it is possible to maintain the entire exercise on a single screen. In some sense, then, scaffolding is addressed by the graphical representation.

Summary and conclusions. The three general techniques for training strategies, subgoals, and goals have several common characteristics. First, each focuses on immersing the student in the task, even when that task may require more skills than the student can currently provide. In each case, the student is required to get through the complete problem-solving process and is supported in this activity through various means. This practice 
addresses head-on the issue of inert knowledge. Any learning that occurs is tied directly to its use in the task context. Cognitive apprenticeship techniques rely on a teacher to provide the scaffolding and coaching. The intelligent tutoring systems described often rely on the availability of hints to prevent the student from getting bogged down. Also, a graphical representation of the problem can aid in reducing working memory demands and providing a broader view of progress.

A second common characteristic of these techniques is a strong reliance on access to a model of skilled performance. Each technique begins with a detailed description of how an expert would solve the problem. The cognitive apprenticeship technique is careful to make that model explicit before practice begins. However, other techniques reveal the expert model only through the hints and advice offered in the course of problem solving. The primary utility of the expert model is to provide a means for diagnosing student performance and guiding instruction. An area that has received considerable attention in ITS development is student modeling. Somehow the tutor needs to keep track of what the student has mastered and what still requires instruction. The student's problem solving performance provides the only evidence of the underlying cognitive skills.

The specific techniques described here also reveal differences in approaches to training. In some cases, the tutor intervenes. In others, the tutor waits to be asked. The issue of timing of feedback is also somewhat controversial. Some believe that feedback on errors must occur as soon as the error is made. Others find value in allowing the student to discover and correct his own errors. These issues and others continue to be debated in the literature and evaluated when ITSs are fielded. In the larger scheme, these differences are less significant than the common characteristics that have been adopted widely for guiding training design.

\section{D.5 Training for Management of Mental Resources}

14. Reduce the need for mental resources. In his early studies (e.g., Schneider \& Shiffrin, 1977), Schneider focused on simple visual search tasks. Since then, more meaningful tasks have been trained. For example, Carlson, Sullivan, and Schneider (1989) trained analysis of single logic gates, and Fisk and Lloyd (1988) trained judgments about the movements of chess pieces. The key in selecting tasks for this type of training is to identify cases in which the required response is consistently mapped to a stimulus. In " other words, the individual makes the same response to a stimulus in all conditions. The link between a printed word and its pronunciation is consistent, but the link between the word and its meaning may be variable. The word "once" is always pronounced the same way but can be synonymous with one time, when, or after.

Training consistently mapped responses can require many hours and thousands of trials of practice and produces a characteristic learning curve (see Figure D2). This curve shows that response speed increases to an asymptotic level (note that the time scale and asymptote level depend on the response being trained). This asymptote (theoretically, the fastest possible response) is a limit on human performance. After automaticity training has been done for the consistently mapped task component, then a second task (perhaps one that is not consistently mapped) can be introduced to train dual-task performance.

Research has shown that with certain tasks (but not all) performance on the automated skill initially degrades when a second task is introduced.

Therefore, additional practice in the dual-task setting is required to regain the performance levels that had been achieved for the single task. In one study, Damos et al. (1981) gave trainees 45 15-minute sessions of single-task training followed by 15 sessions of practice with two simultaneous tasks. Schneider and Fisk (1982) and Fisk and Schneider (1983) also showed that additional dual-task practice is required to regain the performance levels that had been achieved for the single task. Because of the inability to achieve complete transfer to the dual-task setting-that is, because performance of the automated skill suffers in the context of a second task-Schneider and Detweiler (1988) recommend that single-task practice be followed by dual-task practice. Schneider and Detweiler believe that compensatory activities must also be acquired in skill learning. That is, multiple tasks can compete for the same resources, and resource sharing must be developed. By practicing a task under high mental workload conditions, one can acquire the skill in a form that allows efficient execution in the context of performing the integrated task. 


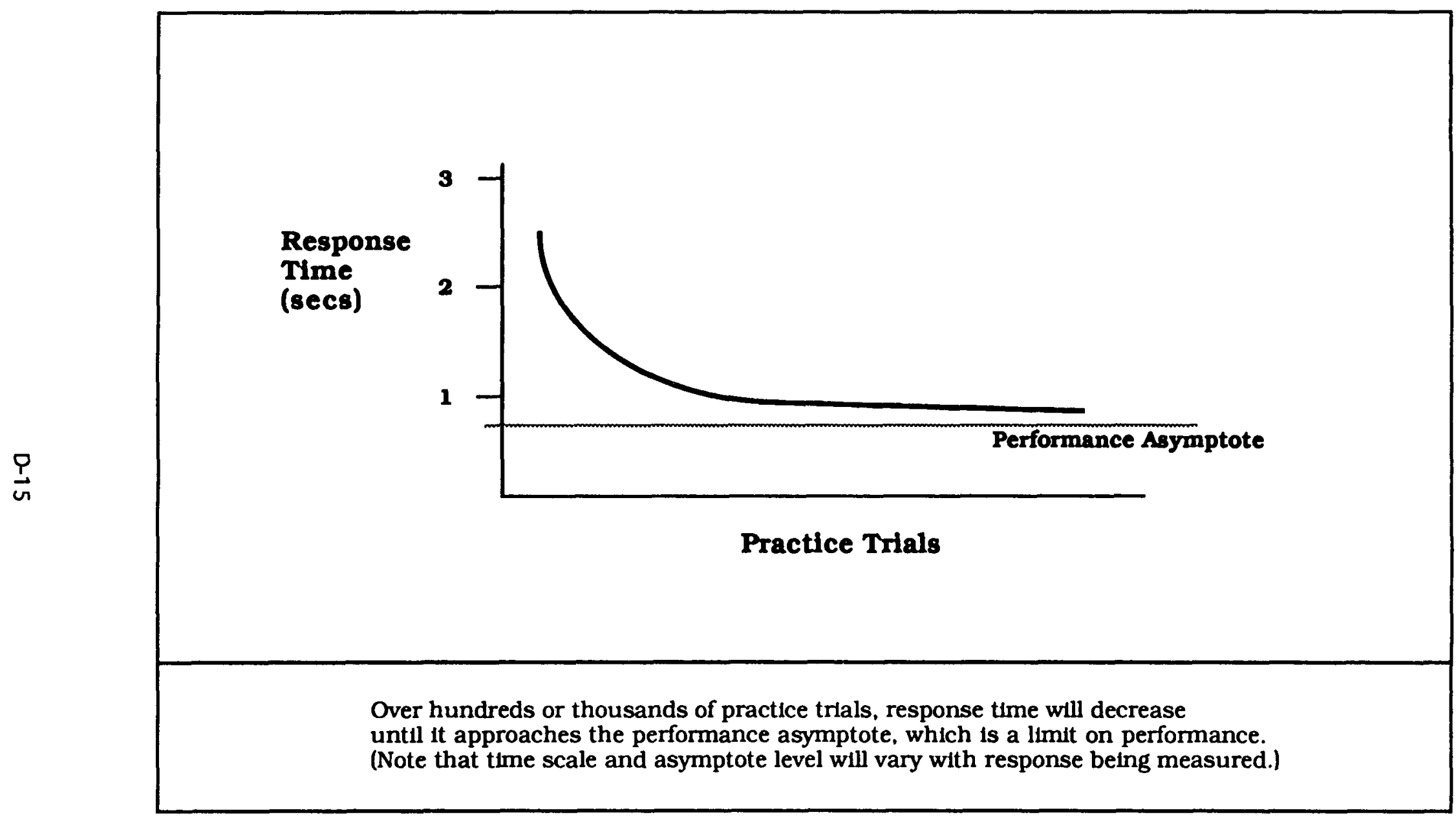

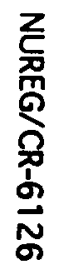

Figure D-2. Standard learning curve for consistently mapped skills. 
15. Eliminate inefficient strategies. Two problems can occur when attempting to integrate skills. First, in the dual-task setting there is the potential for direct interference while processing the two tasks simultaneously. For example, processing strategies for both tasks may require spatial reasoning. Through dual-task training, it may be possible to alter the processing of one of the tasks so that it becomes less dependent on spatial reasoning and more dependent on analytic reasoning. Second, the processing of one task may be less efficient than is possible. In some cases, it may be possible to reduce the number of steps in a cognitive process. Requiring trainees to perform both tasks simultaneously will often push them to discover more efficient processing on their own. To facilitate processing refinement, Schneider (1985) recommends varying the speedaccuracy requirements during practice. In some practice sessions, trainees will be pushed to emphasize speed over accuracy, and may gain in processing efficiency.

In other cases, the inefficiency is not corrected by the trainees and may have to be identified and eliminated through more targeted training. Frederickson et al. (1983) discovered that some poor readers fail to improve reading skills because they rely on a poor strategy. Specifically, these readers use initial and terminal letters in a word to guess at the word, instead of decoding more meaningful word units. Frederickson trained these readers to focus on word units inside the word and was able to improve their reading skills. Without this training intervention, these readers may never have modified their ineffective processing.

Gopher and his colleagues (e.g., Gopher, Weil, Baraket, \& Caspi, 1988) also have explored the importance of training in dual-task settings. From their work in aviation settings, they conclude a) that experience on complex tasks is insufficient to produce effective performance because many performers settle into sub-optimal strategies that support performance that, while adequate, is less than the performer's capabilities; b) through dual-task training, trainees can be taught attention-allocation skills, which can be transferred to new situations.

Summary and conclusions. The ability to perform complex, highly integrated skills often requires that more than one skill be performed simultaneously. In these cases, there is also often a component skill that is cognitive and that requires much of the performer's mental resources. These requirements are especially characteristic of jobs that have some degree of time stress. As one example, advanced control rooms will become more dependent on presenting data and controls through CRTs. This shift in technology will force operators to acquire skills to locate the appropriate display in a large display space and take an action or verify a parameter in a timely way. The interface management skills need to be trained to the point where they do not reduce mental resources that are required for crew coordination and procedure execution. Only by using some of the training techniques described in this section can personnel quickly acquire the ability to integrate lower-level skills with important cognitive skills.

A technique was described to isolate and train consistently mapped component skills. However, to ensure that components are integrated efficiently back into whole-task training and that each component is performed efficiently in the whole-task setting, it is critical to make a transition eventually to dual-task training. Schneider and Detweiler (1988) recommend a scheme to train task components in the sequence $A$, $B, C, A X, B X, C X$, and then $A B C$. In this generic example, $A, B$, and $C$ are components of the integrated task. First, each component is trained in isolation. Then, each component is trained in a dualtask setting, $A X, B X$, and $C X$, where $X$ is a fourth task that may not be a component of the actual task but an artificial task that increases mental workload. This stage of training forces the execution of each of the component tasks to become more efficient. The final stage of training, $A B C$, integrates all three components for realistic practice of the actual task. An alternative is to train pairs such as $A B, A C$, or $B C$ in the second stage instead of combining each component with an artificial task.

\section{D.6 Training a Decision-Making Process}

\section{Train a formal procedure for decision making.}

The multi-attribute utility approach to decision making is one often used in the studies reviewed by Means et al. An MAU approach typically identifies a set of characteristics or features that are critical to the selection and then provides a weight for each alternative on each feature. For example, if one were selecting a refrigerator, one may want to evaluate 
each alternative on its size, color choices, efficiency, reliability, etc. A formal evaluation of the alternatives on these features should lead one to select the "best" alternative. Other computational or analytic models are also used for these types of decisions.

Means et al. found a number of studies in which these decision-making procedures were trained. In each case (e.g., Beatty, 1988; Hayes, 1980), training in formal methods made trainees more consistent and proficient at employing these procedures and more likely to arrive at the prescribed selection. However, some studies point to the difficulties of using these formal methods in a more realistic setting. One concern is time pressure. Zakay and Wooler (1984) trained an MAU procedure for the purpose of selecting an appliance. Their training was successful in that it led to selections that were more optimal from the MAU perspective, but only under conditions of no time pressure. No benefits were found when decisions had to be made under either moderate or severe time constraints. Payne, Bettman, and Johnson (1988) found that, under time pressure, attempts to apply a truncated version of the formal procedure were less successful than the use of a less formal (more heuristic) strategy. There is also evidence that people believe the formal procedures, such as MAU, are too complex, difficult, and time-consuming (Means, 1983). Lave, Murtaugh, and de la Rocha (1984) demonstrated that shoppers are likely to adopt decision-making strategies that avoid computations or a heavy mental burden.

The larger point to be taken from the Means et al. review is that the specific formal procedures trained to individuals do not seem to have much value for the decision-making tasks that occur in the real world. More specifically, MAU and related techniques that focus on formalizing the selection of the best alternative are not useful for many of the important decision-making tasks people face. Means et al. point out that the studies they reviewed did not try to determine how well the newly trained decisionmaking skills transferred to real-world tasks similar in nature to the tasks that are now a primary concern to many researchers-decision-making tasks such as command and control, air traffic control, fire ground commanders, nuclear power plant operations, etc. Thus, there is a need to develop a better understanding of real-world decision-making tasks and train the process underlying those tasks. For example, this report has developed a more realistic approach to describing the decision-making process appropriate to NPPs. Training directed toward a more detailed version of this model would be appropriate.

\section{Reduce decision-making biases. No additionar information on this technique.}

Summary and conclusions. Although a training technique that directly trains a formal decisionmaking process has an intuitive appeal, the studies reviewed by Means et al. (1993) do not present an optimistic picture. There is evidence that people can be trained to apply a formal procedure to be more consistent and more effective at selecting the optimal alternative. However, there are limits on how well those formal procedures are applied in everyday or non-ideal settings (e.g., under time constraints). More important, there are strong limits on the relevance of the formal procedures studied to real-world decisionmaking tasks. Thus, training the MAU technique or a related technique would provide little benefit to nuclear power plant decision-making performance. Means et al. also present evidence that non-specific training on eliminating decision biases is very limited.

These conclusions do not suggest, however, that directly training the appropriate decision-making process will not be beneficial. The earlier sections of this report have attempted to reveal through analysis that the decision-making task required for nuclear power plant operations, and SAM operations, is far more complex than the decision-making tasks that strive to select the best alternative. Further, many of the training techniques described here have been effective in training the cognitive processes underlying skilled performance of the decisionmaking or problem-solving task.

\section{D.7 Training Team Skills}

In the analysis and training of cognitive skills, there is a substantial literature on skills defined at the individual level but relatively little on skills defined at the team or crew level. Part of the reason for the relative lack of applicable information is a distinction that has developed between two types of teams involved in decision making: ad hoc teams and teams from actual work settings. The majority of published research relates to the performance of ad hoc teams, 
which are groups of people brought together temporarily to perform, in many cases, an artificial decision-making task. That is, these teams are meeting for the first time to perform a task that is novel to them. Significantly fewer studies have been conducted to investigate the performance of existing decision-making teams in realistic work settings-for example, commercial aviation cockpit crews, command and control crews, nuclear power plant operating crews. While there are certainly lessons to be learned from the research on ad hoc teams (see Driskell \& Salas, 1992), that work is often not directly relevant to the training of "real" teams.

One development in the study of individual cognitive skills that profoundly influenced techniques for training was the analysis of skilled performance. That is, the development of a description of expertise and a theory of cognitive skill acquisition for an individual led to a number of innovations in cognitive skill training. Analogous efforts at the team level have a much shorter history. Thus, although skilled teams are often easily distinguished from poorly performing teams, a generic (i.e., independent of domain) description of skilled team performance has yet to find broad acceptance. After such a description has been established, the goals of team training and appropriate training techniques can become more clear.

An intensive research program, sponsored by the Naval Training Systems Center (NTSC) and NASAAmes, has recently undertaken the task to describe skilled crew performance. Franz, Prince, CannonBowers, and Salas (1990) determined that in the area of aviation crews the following seven performance dimensions should guide descriptions of team performance:

- Communication

- Situational awareness

- Decision making

- Mission analysis

- Leadership

- Adaptability / Flexibility

- Assertiveness

In a project sponsored by the NRC, a similar analysis was done for control room operator crews (see Montgomery et al., 1992). In this case, six performance dimensions were identified for describing team performance:
- Communication

- Task coordination

- Maintaining task focus in transitions

- Adaptability

- Openness / Participation

- Team spirit

While there are differences between these two lists, there are also some striking similarities. In both cases, communication skills are considered to be critical for successful team performance. Three important elements of communication are the identification and resolution of errors; information exchange that is accurate, clear, timely, and appropriate; and statements that are motivational or praising of other crew members (Oser, McCallum, Salas, \& Morgan, 1989). These elements have a clear role in performance of the complex decision-making tasks described in this report. Also, each set of performance dimensions addresses both task-related issues (such as communication of accurate information) as well as teamwork issues (such as leadership, team spirit, and the ability to express one's opinion to the group openly).

18. Employ a behavior-based ACT technique. A concern with these programs as they have been developed is the vagueness with which team skills are sometimes defined. For example, the team performance dimensions are not always tied to the same behaviors across training programs. More generally, trainers may not understand how to rate specific behaviors in terms of the team skills that are being rated. According to Helmreich and Wilhelm (1986), LOFT evaluators need to be familiar with the desired behaviors in the context of the scenario and trained to observe team behaviors and provide specific feedback. Even crew members' own ratings of their performance are inaccurate unless they understand the specific behaviors expected in the training situation. Because of these difficulties, a behavior-based technique has been developed to make explicit the types of behaviors that indicate good team skills.

According to Prince et al., a behavior-based program seeks to identify particular behaviors within team skill dimensions that result in effective team performance. These are the behaviors that should be trained and evaluated. In an NTSC technical report (Oser, McCallum, Salas, and Morgan; 1989), 
researchers identified behaviors tied to team skill dimensions while observing Navy Tactical Teams. Examples of behaviors that were characteristic of more effective teams were:

- Helped another member who was having difficulty with a task

- Made positive statements to motivate the team

- Coordinated gathering of information in an effective manner

A similar approach was taken by Montgomery et al. (1992) in observing nuclear power plant operating crews. In both cases, specific behaviors were tied to team skill dimensions. As this work develops it will eventually become possible to identify behaviors that are specific to a training scenario. For example, as new information becomes available to a crew member in a scenario, training evaluators can watch for indications that that crew member is using effective communication skills. Thus, after specific behaviors are linked with team skill dimensions, those behaviors can be embedded in meaningful scenarios.

Prince et al. have recommended two basic techniques for training effective behaviors with behavior-based ACT. First, effective behaviors can be modeled. That is, a crew could rehearse a response to a training scenario so that critical behaviors are demonstrated. Then, the instructor could work with a new crew to point out the effective behaviors as they view a videotape of the model crew. A second technique is active practice of scenarios that present opportunities for effective team behaviors. Prince et al. suggest that a high-fidelity, full-scope simulation may not be required for crews to practice coordination skills and other team skills. NTSC has developed a low-fidelity aircrew environment that they believe supports crew training well. However, the need for a high-fidelity environment may depend somewhat on the domain. The question of appropriate fidelity level requires further study.

\section{Develop "shared mental models". The value of} shared mental models is not thoroughly established. There is a belief by some (e.g., Kleinman \& Serfaty, 1989) that in some cases skilled teams require less overt or explicit communication because crew members have developed strongly overlapping mental models. Kleinman and Serfaty (1989) observed a team involved in distributed decision making and manipulated the performance demands (e.g., time stress). They found that overt communication and coordination decreased as workload increased. As the demands on each crew member increased, the team grew more silent. Kleinman and Serfaty interpreted this finding as an "implicit coordination" strategy, where individuals use their mental models, instead of explicit communication, to anticipate needs and demands of other crew members.

While the notion that highly skilled teams have less need for overt communication and coordination is an appealing one, too little data exist at this point to draw strong conclusions. However, even if there is no desire to reduce overt communication among team members, there is utility in developing strong compatibilities in the crew's understanding of the task, the equipment, and the roles of team members. In this situation, crew communication may be more efficient or more closely tied to goals and strategies. Indeed, another hypothesis (Orasanu, 1990) is that with shared mental models communication is qualitatively different for highly skilled teams, but is not significantly reduced.

Summary and conclusions. This section on training crew skills is certainly more speculative than other sections about identifying training techniques that can train cognitive skills effectively. There is a significantly shallower and more tenuous understanding of the crew skills required for skilled performance when compared to our understanding of individual skills. Some consensus has been developing in the last several years, but these initial studies often describe crew performance dimensions at a high level (e.g., communication, coordination), and performance descriptions are, therefore, difficult to compare across studies and domains. There are currently several efforts to identify more specific behavioral indices of the team skills. As specific behaviors are identified, they are being incorporated into the ACT approach to training. ACT begins with awareness training but then quickly progresses to scenario-based practice sessions in which crew members are presented with challenging situations that demand effective crew skills. Instructors focus on identifying areas where the appropriate behaviors are missing. The belief is that effective instruction on team skills will occur when crew members can view their videotaped performance and the appropriate behaviors can be modeled or explained to guide evaluation. Initial evaluations of this type of training 
are positive, but more studies are required to draw strong conclusions.

Another technique that deserves some attention is the notion of training crews to develop compatible or shared mental models. The discussion identified the potential value in this concept, but specific training techniques have yet to be evaluated. This is an area to monitor as programs are developed and tested in aircrew and combat information center settings.

Two other points regarding effective team training require emphasis. First, the common wisdom is that team training is most effective after individual skills are mastered. Salas, Dickinson, Converse, and Tannenbaum (1992) identify a number of studies that support the value of this training progression.

Certainly, from the perspective of the management of mental resources in training, if the instructor desires to focus attention on team skills, the individual skills must be mastered.

Second, the primary developers of the behavior-based techniques, both for aircrews and nuclear power plant operators, have devoted significant resources to instructor training. An instructor's ability to identify the appropriate team behaviors in the context of a scenario is not easily acquired. Also, because the target of instruction is less well defined, evaluation and remediation are difficult. The delineation of instructor skills must improve to facilitate the training of effective instructors. 